\title{
Investigation of the electron and thermal transport in rare-earth nitrides
}

\author{
By
}

Tanmay Maity

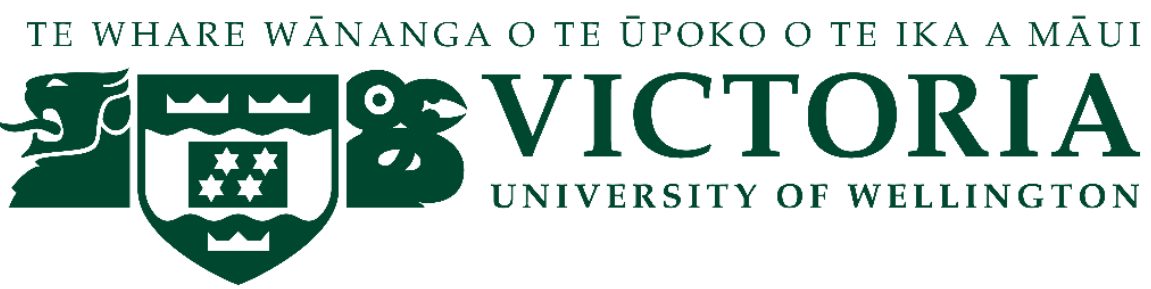

\begin{abstract}
A thesis
submitted to the Victoria University of Wellington in fulfillment of the requirements for the degree of

Doctor of Philosophy in Physics
\end{abstract}

Victoria University of Wellington 

All science is either physics or stamp collecting

- Ennest Rutherfard - 



\section{Abstract}

Gadolinium nitride $(\mathrm{GdN})$ and samarium nitride $(\mathrm{SmN})$ have been widely studied to understand their ferromagnetic ordering and electronic structure, and for their promise in spintronics applications. This thesis presents experimental magnetotransport studies of GdN and SmN films in which experimental results have been compared with the existing band structure calculation. Three GdN films have been prepared in different conditions, among them two films are epitaxial quality and one film is polycrystalline in nature, and two films of $\mathrm{SmN}$ were also studied. Their magnetic properties were probed by SQUID magnetometry and they are found to be ferromagnetic. The transition temperature differs from sample to sample and this behaviour has been attributed to the presence of magnetic polarons that nucleate around nitrogen vacancies and give rise to an inhomogeneous ferromagnetic state.

The charge transport results have been discussed for all GdN and SmN films. A full set of charge/heat transport results are obtained on only one epitaxial GdN. The difference of resistivity among these samples is noticeable. The Hall effect results show the presence of different carrier concentration with at most only weak temperature dependence. We also have noticed the presence of anomalous Hall effect in the paramagnetic region for a lowerconcentration epitaxial GdN.

The thermopower in both $\mathrm{GdN}$ and $\mathrm{SmN}$ was measured to provide further insight into the material's electronic properties. In this thesis we present the first experimental investigation of the thermopower of epitaxial gadolinium nitride and samarium nitride films, measured using an experimental set-up designed for measuring the temperature dependent thermopower of thin films. Our result shows a negative thermopower for both $\mathrm{GdN}$ and $\mathrm{SmN}$ films and simple, though strong temperature dependence. At low temperatures we observe a peak near the ferromagnetic transition temperature in GdN. The results are interpreted in terms of the diffusion thermopower.

Overall the results suggest that the nitrogen vacancy concentration controls the carrier concentration and plays a significant role towards the transport properties. We conclude that all films are either heavily, moderately or weakly doped semiconductors with a metallic characteristic. 


\section{Acknowledgements}

Firstly, it is a great pleasure to thank my two supervisors, Joe Trodahl and Ben Ruck, for their guidance, encouragement, patient assistance and helping me to understand the experimental as well as conceptual issues throughout this research project. I am especially grateful for all their stellar efforts in supervising me. I am in no doubt that I could not have asked for better supervisors. Without their help, this thesis could not have been created.

Two other group members worthy of mention, and who have been greatly assisting me are Simon Granville and Franck Natali. Whenever I had a problem in understanding the experimental set-up or analysing the data, they were always available to help me. In particular, I am extremely grateful to Simon Granville and Shen Chong for hands-on training in using the PPMS and SQUID for magnetic and transport measurements at the Robinson Research Institute (RRI), Gracefield.

I would like to express my sincere gratitude to John Kennedy and Peter Murmu for giving me access to the RBS and Hall effect measurements at GNS Science, Gracefield. I also extend my thanks to Natalie Plank for allowing me to use the clean room. I have greatly benefited from working with all spintronics group members, especially with Harry Warring, Jay Ross Chan, Luis Figueras, James McNulty, Chang Min Lee, Eva Anton and Bart Ludbrook.

I am very grateful to Jonathan Halpert who gave me the opportunity to work in an exciting solar cell research project and engaged me in his chemistry lab as a research assistant, which enabled me to develop both scientific and management skills.

I am fortunate enough to have engaged in several interesting physics discussions with my former supervisors, Ram Janay Choudhary and S. P. Sanyal during my Ph.D. tenure, and thank them for hosting me while visiting their labs.

Apart from my research group members, I have thoroughly enjoyed the discussions on various topics with my well-wishers and other friends - the time spent with the family of Jay Shaw, and Prabir Da and Nirupam Da will be unforgettable.

Periodic discussions with Satya, Bhakta, Praveen, Srikanth, Saptorshi, Vikram, Rajdeep, Rohit, Nayan, Partha $\mathrm{Da}$ and some other friends are quite memorable, and their positive wishes will always mean a lot to me. 
I could not have completed this work without the financial support from the Victoria University of Wellington and the MacDiarmid Institute for Advanced Materials and Nanotechnology. I wish to extend my heartfelt thanks for the scholarship which enabled me to complete my research work and produce this thesis. I would also like to thank all the staff of the School of Chemical and Physical Sciences for their sincere help.

I am forever grateful to my loved ones, who were always with me on this long and winding journey. I thank my parents for supporting and encouraging me throughout this course, and their inspiration to finish up my Ph.D. Your love and generosity have helped make me the person I am today. Thanks also to my late grandfather who taught me to become a good person and encouraged me to pursue higher studies. I would also like to thank my lovely sister for her encouragement and tremendous moral support on my Ph.D. journey.

Finally, I would like to express my deepest thanks to my wife, for her love, affection, support, sacrifices, encouragement and taking care me during this Ph.D. adventure. 


\section{Table of Contents}

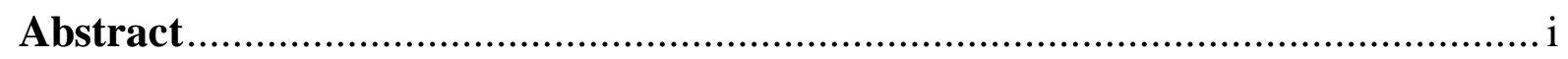

Acknowledgements ...............................................................................

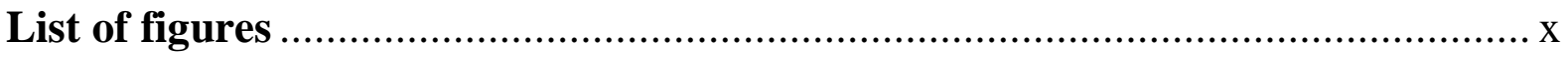

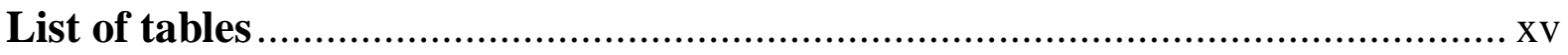

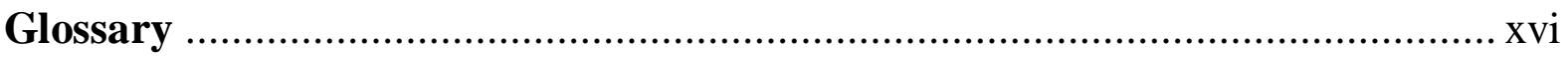

1 Introduction and literature review ........................................................ 1

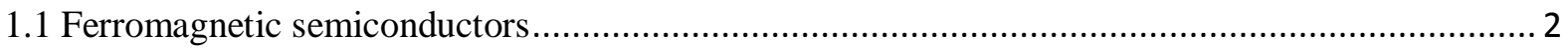

1.2 Importance of rare-earth $4 f-5 d$ orbitals in magnetic and transport properties ............................ 4

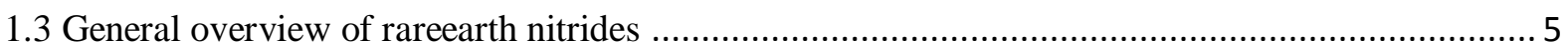

1.3.1 History and fundamentals of rare-earth nitrides ..................................................... 5

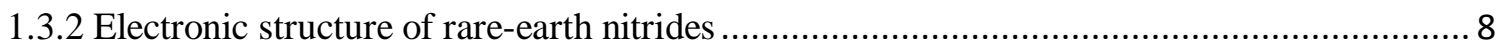

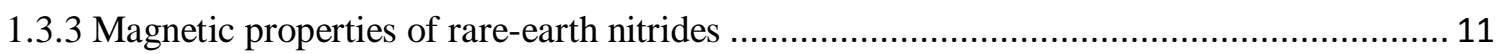

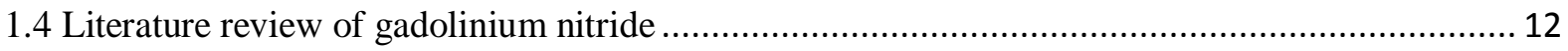

1.5 Magnetic and electronic properties of samarium nitride ...................................................... 17

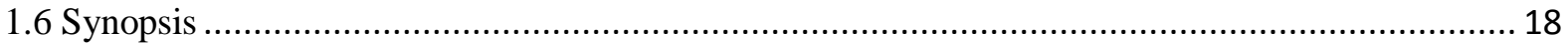

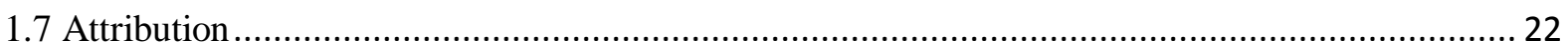

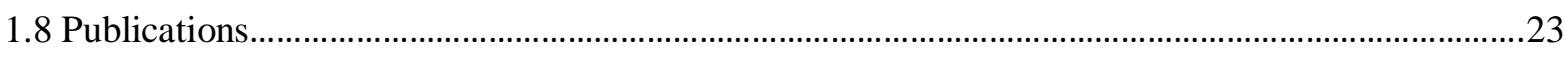

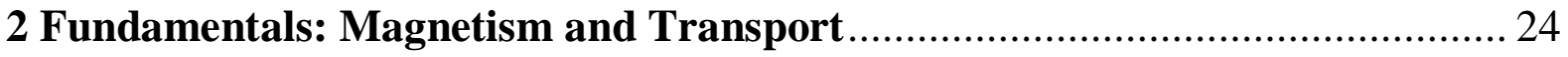

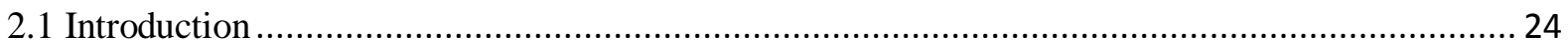

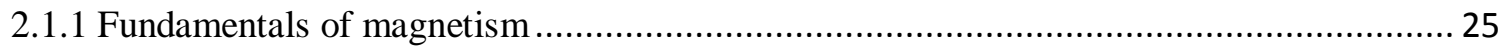

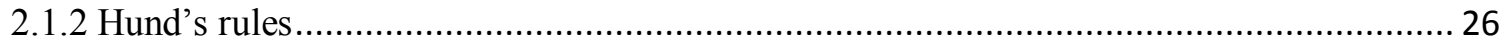

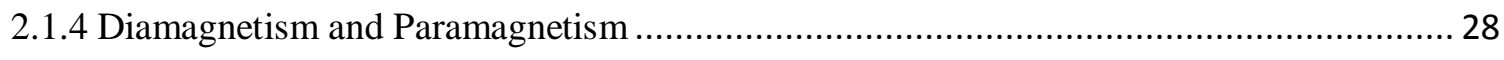

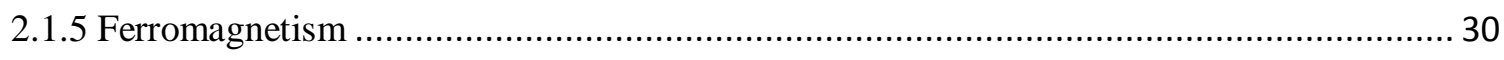

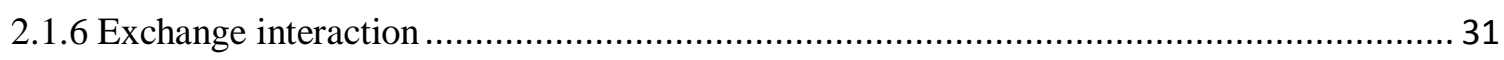

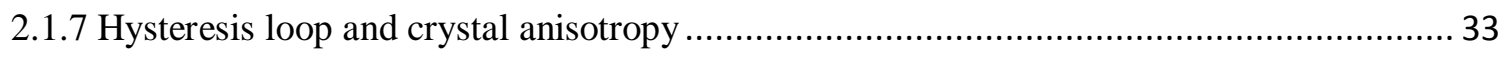


2.1.8 Shape anisotropy

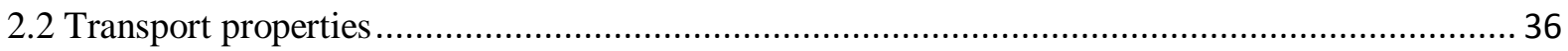

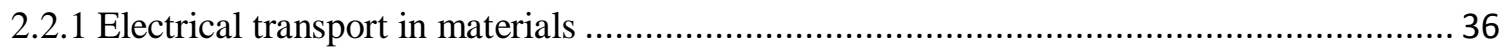

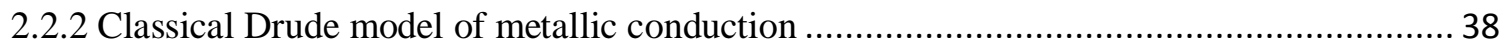

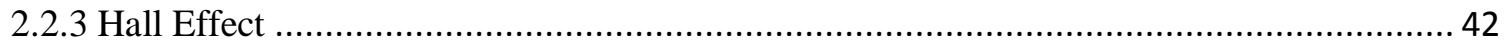

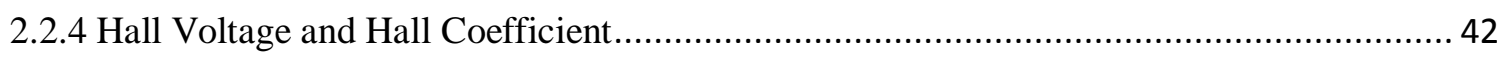

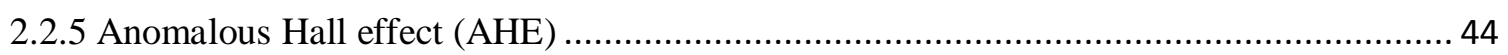

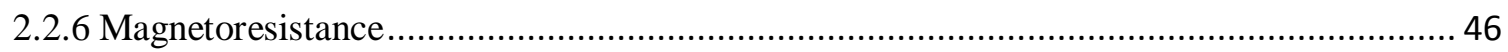

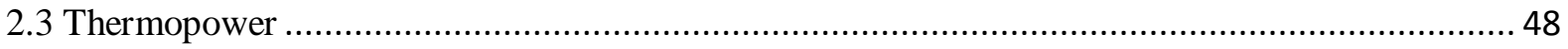

2.3.1 Definition and the physical origin of thermopower ..................................................... 48

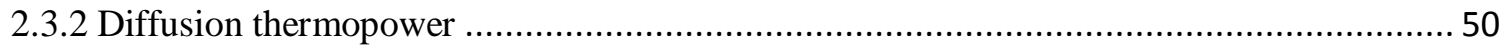

3 Sample preparation and characterisation ………….................................... 52

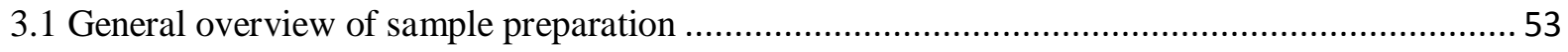

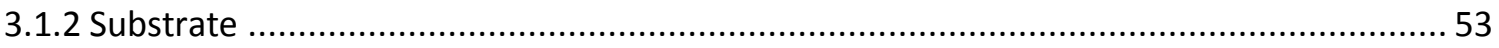

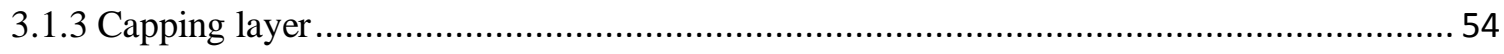

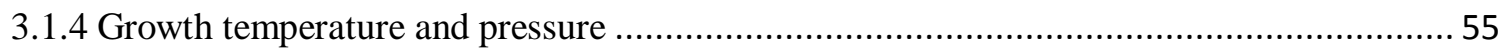

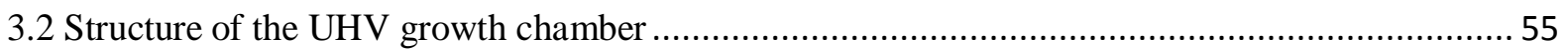

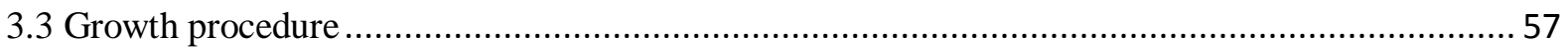

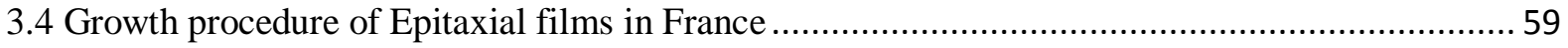

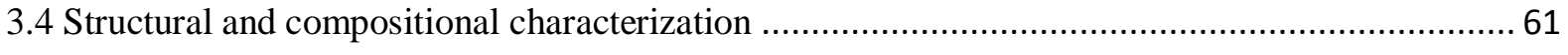

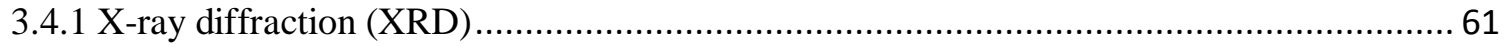

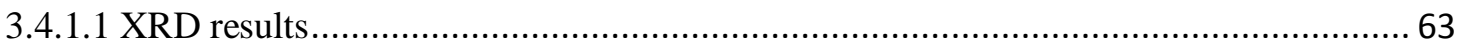

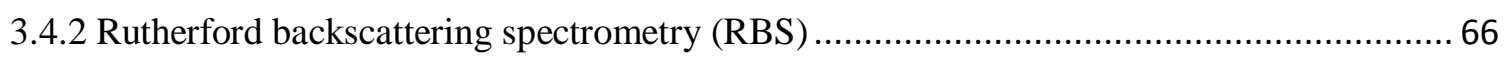

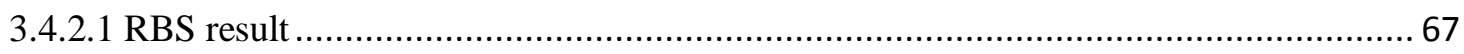

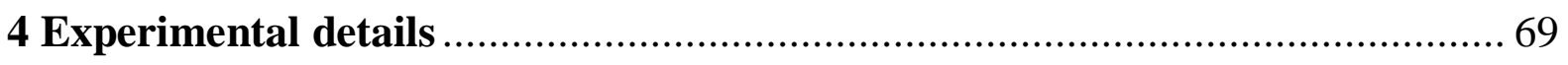

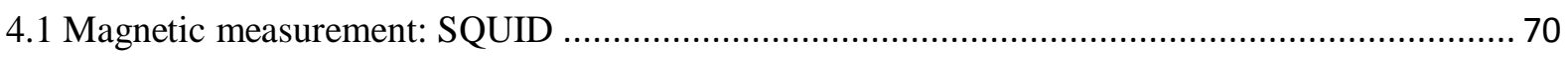

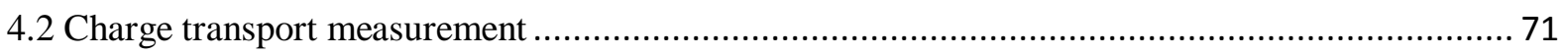

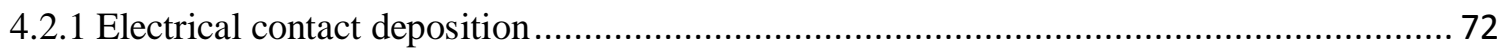


4.2.3 Resistivity and magnetoresistance measurements .................................................... 74

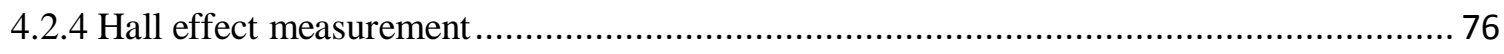

4.3 Charge/heat transport measurement (Thermopower measurement) ….................................... 78

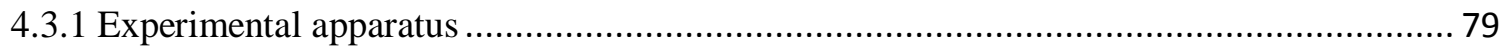

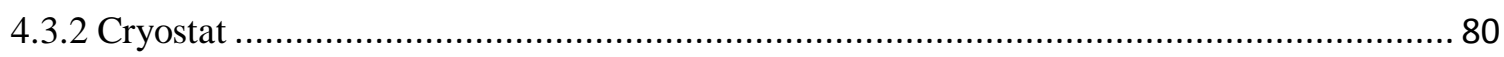

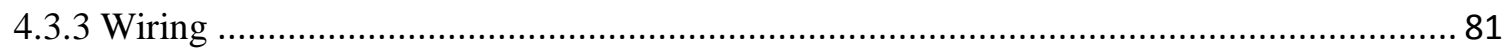

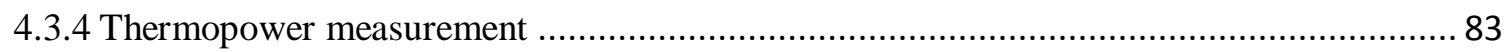

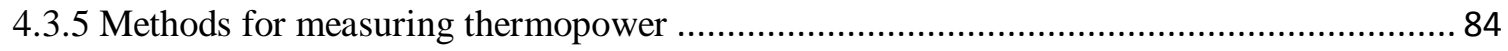

4.3.6 Thermal voltage measurement technique inside the cryostat ........................................ 85

4.3.7 System calibration, substrate contribution and uncertainty measurement .......................... 88

\section{Magnetotransport properties of high carrier concentration epitaxial}

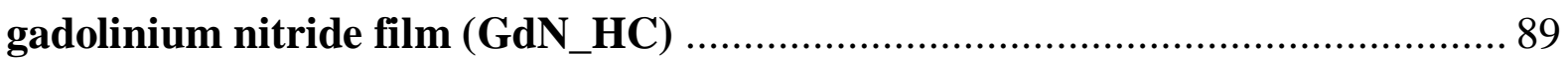

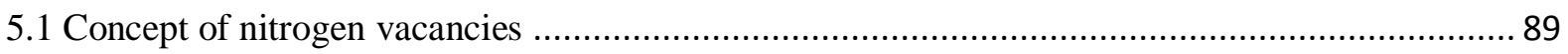

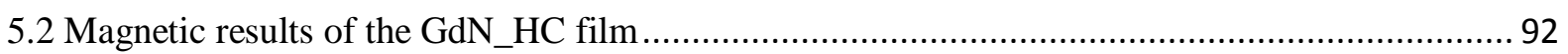

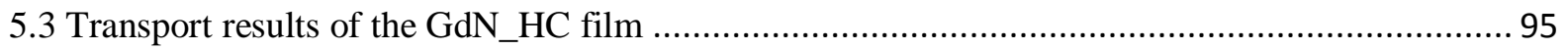

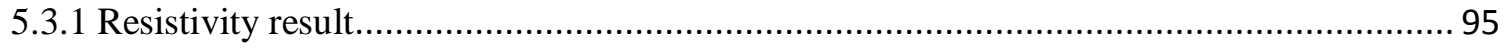

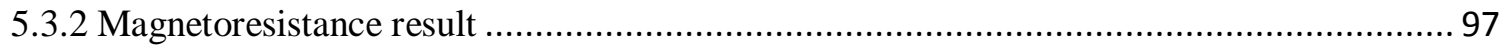

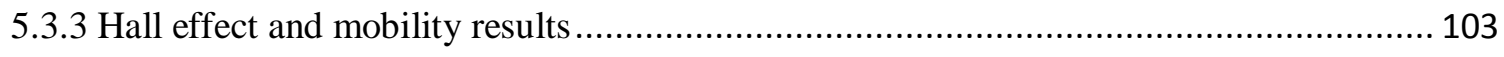

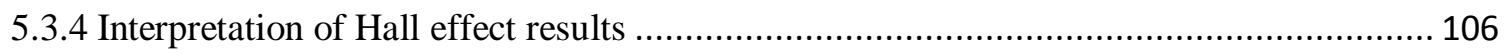

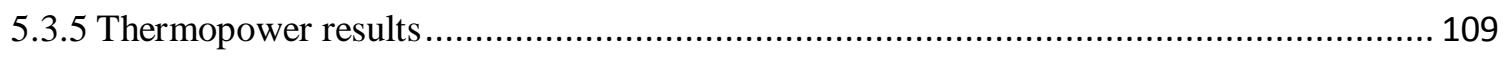

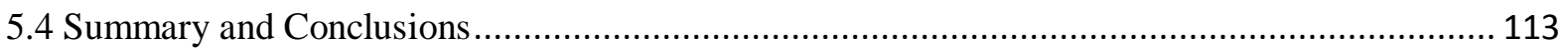

6 Magnetotransport properties of low carrier concentration epitaxial gadolinium nitride film (GdN_LC) .......................................................................... 114

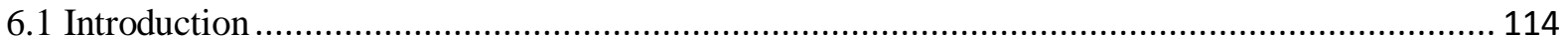

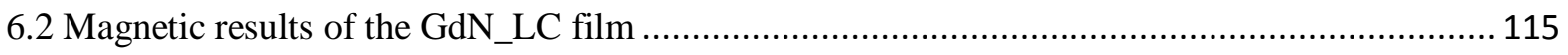

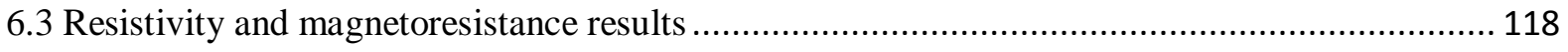

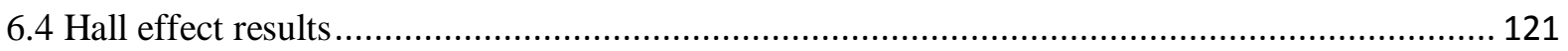


6.4.1.1 Fitting in the ferromagnetic region ................................................................ 123

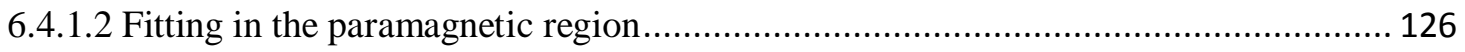

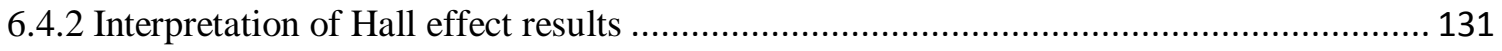

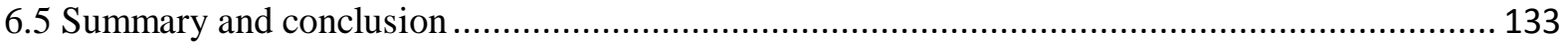

7 Magnetotransport properties of a polycrystalline gadolinium nitride film .... 134

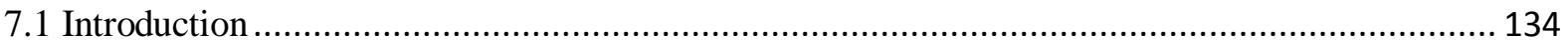

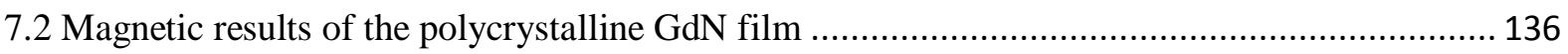

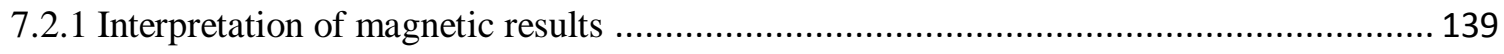

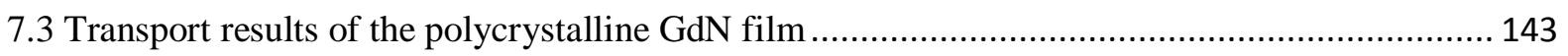

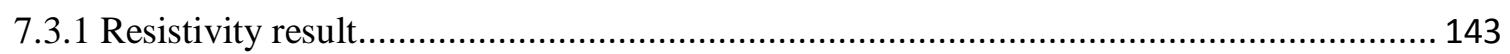

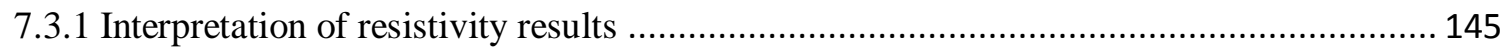

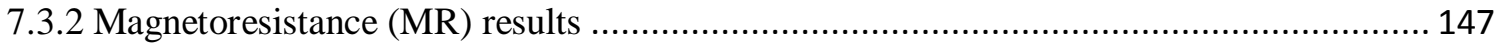

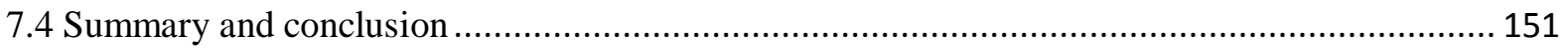

8 Magnetotransport properties of samarium nitride films ............................... 153

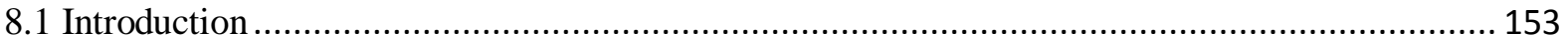

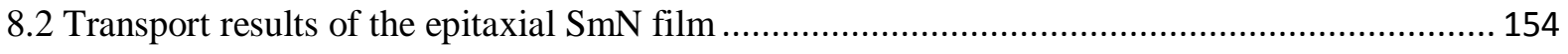

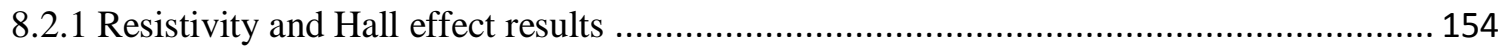

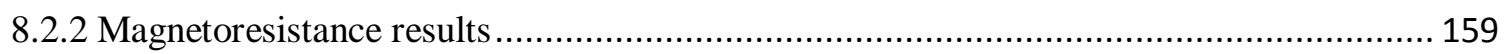

8.2.3 Thermopower results of the epitaxial and polycrystalline $\mathrm{SmN}$ films ............................ 162

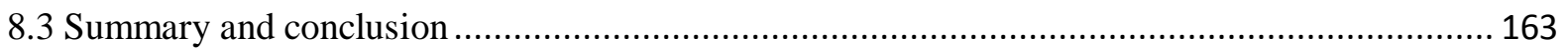

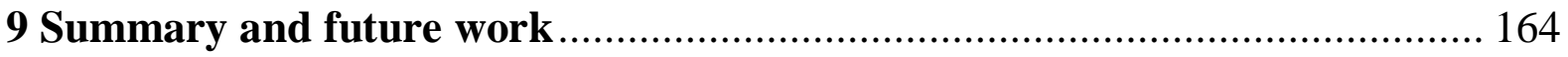

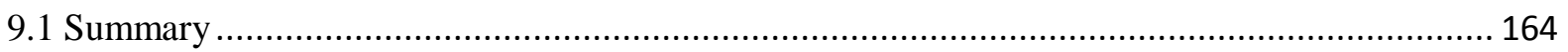

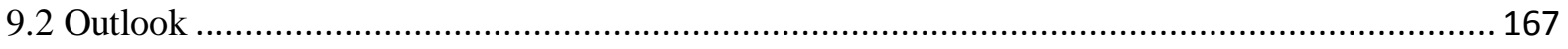

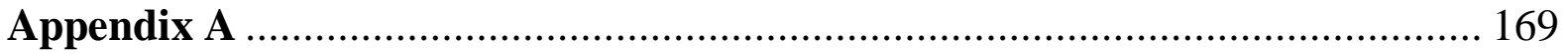

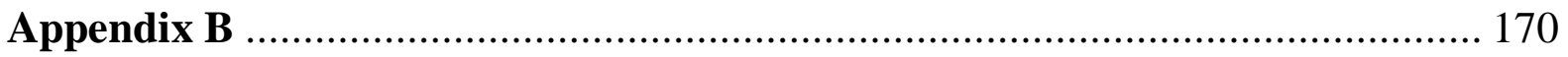

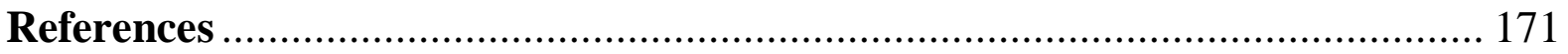




\section{List of figures}

Figure 1.1: Schematic of the common rocksalt crystal structure of the RENs. The small deep lilac spheres represent the RE cations while the large cadet blue spheres are the $\mathrm{N}$ anions.

Figure 1.2: $\mathrm{LSDA}+U$ calculation of the GdN band edges in the ferromagnetic state with $U f$ (left, semimetallic) and $U f+U d$ (right, semiconducting) corrections applied to the $f$ - and $d$-orbitals, respectively. Solid lines: majority-spin; dashed lines minority-spin. Figure adapted from: Larson and Lambrecht [55].

Figure 1.3: The calculated band structure of GdN (adapted from [116]). 16

Figure 2.1: Total magnetic moment of rare-earth nitride series. The orbital and spin components of the magnetic moment are indicated by red and blue colours, respectively. This figure is reproduced from Larson et al. [25).

Figure 2.2: Hysteresis loop and domain structure formation for a ferromagnet below $T c$ at varying magnetic field strength (Reproduced from [132]).

Figure 2.3: Simplified illustration of the electronic energy levels for a metal, semiconductor and insulator. $\mathrm{E}_{\mathrm{F}}$ and $\mathrm{E}_{\mathrm{g}}$ represent the Fermi level and band gap, respectively.

Figure 2.4: Geometry of the Hall effect. The Lorentz force $-e v \times B$ on the electrons is balanced by the force $-e E H$ due to the Hall field $E H$

Figure 2.5: A thermoelectric circuit. .48

Figure 2.6: Simplified illustration of the diffusion of electrons caused by a temperature gradient. An electrically isolated slab of material is heated on the left $(\Delta T)$ causing a net flux of electrons towards the right. 50

Figure 3.1: Schematic diagram of an ultra-high vacuum deposition chamber at thin film lab, 56

Figure 3.2: XRD scan of an epitaxial GdN film grown on an AlN template on a silicon substrate at 64

Figure 3.3: XRD scan of a polycrystalline GdN film grown on a sapphire substrate at room temperature.

Figure 3.4: XRD scan of the epitaxial SmN film grown on an YSZ substrate at high temperature.... 66 
Figure 3.5: RBS spectrum of the polycrystalline GdN film.

Figure 4.1: Illustration of wire connection for resistivity measurement

Figure 4 2: REN sample was mounted on a resistance bridge sample holder board inside the PPMS rotator probe for four-wire resistance measurements.

Figure 4.3: Schematic diagram of the closed cycle cryostat used for thermopower measurements. .. 80

Figure 4.4: DT - 470 - CU silicon diode thermometer [Adapted from 170]. 82

Figure 4.5: Block diagram of the thermopower rig.

Figure 4.6: Thermopower circuit.

Figure 4.7: Thermopower set-up inside the cryostat in the low temperature lab. 86

Figure 5.1: (a) Schematic band diagram of GdN. (b) Schematic density of states, where the Fermi level is at $\mathrm{E}=0$ when $T>T c$, no spin spliting. (c) Fermi level is at $\mathrm{E}=0$ when $T<T c$, less nitrogen vacancy concentration. (d) Fermi level is at $\mathrm{E}=0$ when $T<T c$, high nitrogen vacancy concentration. Spin-up $($ red color $)=$ majority-spin, spin-down $($ blue color $)=$ minority-spin .

Figure 5.2: Magnetisation curve and first derivative (inset) of the epitaxial GdN film_HC as a function of temperature.

Figure 5.3: Magnetisation data of the epitaxial GdN_HC film as a function of field at $5 \mathrm{~K}$. Inset: the hysteresis loop of the epitaxial GdN_HC film at $5 \mathrm{~K}$.

Figure 5.4: Temperature dependent resistivity and magnetisation at 250 Oe of the epitaxial GdN_HC film. Inset: Temperature derivative of the resistivity of the same GdN film.

Figure 5.5: Magnetoresistance of the epitaxial GdN_HC film in applied fields perpendicular to the film surface. 98

Figure 5.6: Magnetoresistance of the epitaxial GdN_HC film in applied fields parallel to the film surface.

Figure 5.7: Temperature dependent magnetoresistance at different field orientation of the epitaxial GdN_HC film at $\pm 8 \mathrm{~T}$. 100 
Figure 5.8: Magnetoresistance of the epitaxial GdN_HC film at $10 \mathrm{~K}$ measured with the applied magnetic field perpendicular (black line) and parallel (red line) to the film surface. Inset: magnetisation as a function of applied magnetic field parallel to the film surface at $10 \mathrm{~K}$. 101

Figure 5.9: Magnetoresistance of the epitaxial GdN_HC film at $3 \mathrm{~K}$ measured with the applied magnetic field perpendicular (black line) and parallel (red line) to the film surface. 102

Figure 5.10: Hall resistance of the epitaxial GdN_HC film as a function of applied magnetic field at different temperatures.

Figure 5.11: Inverse of the slope as a function of temperature retrieved from the Hall effect data. . 104

Figure 5.12: Mobility as a function of temperature of the epitaxial GdN_HC film. 105

Figure 5.13: Schematic illustration of the density of states of GdN assuming an imprity level lying above the conduction band minimum. The shaded regions represent occupied states.

Figure 5.14: The band structure of GdN calculated within the LSDA $+U$ approximation [116]... 108

Figure 5.15: Temperature dependent thermopower of the epitaxial GdN_HC film.

Figure 6.1: Magnetisation curve and Curie-Weiss fitting (inset) of the epitaxial GdN_LC film as a function of temperature.

Figure 6.2: Magnetisation data of the epitaxial GdN_LC film as a function of field at $10 \mathrm{~K}$.

Figure 6.3: Temperature dependent resistivity and magnetisation of the epitaxial GdN_LC film... 118

Figure 6.4: Temperature and field dependent resistivity of the epitaxial GdN_LC film.

Figure 6.5: The resistivity of the two epitaxial films at the intermediate temperature of $30 \mathrm{~K}$ plotted vs the departure of the magnetisation from its saturation value.

Figure 6.6: Hall resistance as a function of applied magnetic field for the epitaxial GdN_LC film. 122

Figure 6.7: In-plane and out-of-plane magnetic data for the shape anisotropy correction.

Figure 6.8: Experimental and fitting data of Hall resistance as a function of field at $10 \mathrm{~K}$ and $30 \mathrm{~K}$ (inset).

Figure 6.9: Estimated magnetisation as a function of temperature and field for the epitaxial GdN_LC film.

Figure 6.10: Comparison of experimental and fitted Hall data of the epitaxial GdN_LC film in the paramagnetic region. 128 
Figure 6.11: The uncorrected and corrected inverse of the slope as a function of temperature for the epitaxial GdN_LC film. 130

Figure 6.12: Mobility as a function of temperature of the epitaxial GdN_LC film. 131

Figure 7.1: Magnetisation curve and Curie-Weiss fitting (inset) of the polycrystalline GdN film as a function of temperature. 136

Figure 7.2: Magnetisation data of the polycrystalline $\mathrm{GdN}$ film as a function of field at $5 \mathrm{~K}$. 137

Figure 7.3: Coercive field of the polycrystalline GdN film as a function of temperature. 138

Figure 7.4: A diagram of the magnetic ordering by polarons, for low and high carrier concentration GdN films. 140

Figure 7.5: Temperature dependent resistivity and magnetisation of the polycrystalline GdN film. Inset: Arrhenius plot at low temperature 143

Figure 7.6: Resistivity of the polycrystalline GdN film at different fields as a function of temperature.

Figure 7.7: Magnetoresistance for the polycrystalline GdN film in applied fields perpendicular (out-ofplane) to the film surface at different temperatures.

Figure 7.8: Magnetoresistance for the polycrystalline $\mathrm{GdN}$ film in applied fields parallel (in-plane) to the film surface at different temperatures. 148

Figure 7.9: Magnetoresistance of the polycrystalline $\mathrm{GdN}$ film at $10 \mathrm{~K}$ measured with the applied magnetic field perpendicular (black line) and parallel (red line) to the film surface. The hysteresis loop under the perpendicular applied field has a coercive field $0.3 \mathrm{~T}$ while under the parallel applied field it is only about $0.03 \mathrm{~T}$. Inset: magnetisation as a function of applied magnetic field parallel to the film surface, where the coercive field estimated from the hysteresis loop is approximately $0.03 \mathrm{~T}$. 149

Figure 7.10: Comparison of high-field MR results of the epitaxial GdN_HC film in perpendicular field and the polycrystalline GdN film in perpendicular and parallel field orientations. 150

Figure 8.1: Temperature dependent resistivity of the epitaxial $\mathrm{SmN}$ film. The inset shows the temperature derivative of the resistivity.

Figure 8.2: Hall resistance as a function of applied magnetic field for the epitaxial SmN film. 155

Figure 8.3: Inverse of the slope of the epitaxial $\mathrm{SmN}$ film at different temperatures. 156 
Figure 8.4: The schematic band structure pattern of $\mathrm{SmN}$ film drawn from the reference [25].

Figure 8.5: Mobility as a function of temperature of the epitaxial $\mathrm{SmN}$ film.

Figure 8.6: Magnetoresistance for the epitaxial $\mathrm{SmN}$ film in applied fields perpendicular to the film surface at different temperatures. 159

Figure 8.7: Magnetoresistance for the epitaxial $\mathrm{SmN}$ film in applied fields parallel to the film surface at different temperatures. 160

Figure 8.8: Magnetoresistance of the epitaxial $\mathrm{SmN}$ film at $3.5 \mathrm{~K}$ measured with the applied magnetic field perpendicular (black line) and parallel (red line) to the film surface. 161

Figure 8.9: Temperature dependent thermopower results of the epitaxial $\mathrm{SmN}$ film. 162

Figure A.1: Thermopower of manganin at various temperatures (Reproduced from [172]). 169

Figure B.1: Voltage and Temperature relationship for DT $470 \mathrm{CU}$ thermometer for the thermopower measurement (Adapted from [170]). 170 


\section{List of tables}

Table 1.1: Experimental and calculated lattice constants of rare-earth nitrides (adapted from [24, 25]).

Table 3.1: Growth parameters for $\mathrm{GdN}$ and $\mathrm{SmN}$ films. 


\section{Glossary}

RE Rare-earth

REN Rare-earth nitrides

FCC Face - centred cubic

DMS Diluted magnetic semiconductor

DOS Density of states

LDA Local density approximation

LSDA Local spin density approximation

LSDA $+U \quad$ Local spin density approximation plus Hubbard U correction

QSGW Quasi-particle self-consistent GW (G and W stand for Green's function and screened Coulomb interaction)

APW Augmented plane wave method

SIC Self - interaction correction

MFT Mean-field theory

RKKY Rudermann - Kittel - Kasuya - Yosida

XAS X-ray absorption spectroscopy

XMCD X-ray magnetic circular dichroism

FMR Ferromagnetic resonance

MBE Molecular beam epitaxy

UHV Ultra high vacuum

PLD Pulsed Laser Deposition

YSZ Yittria stabilized zicronia

XRD X-ray diffraction

SEM Scanning electron microscope 
RHEED Reflection high energy electron diffraction

RBS Rutherford backscattering spectroscopy

VSM Vibrating sample magnetometer

SQUID Superconducting quantum interference device

FC Field cooled

ZFC Zero field cooled

$T_{c} \quad$ Transition temperature

PPMS Physical property measurement system

AHE Anomalous Hall effect

EHE Extraordinary Hall effect 



\section{Chapter 1}

\section{Introduction and literature review}

This is an experimental thesis where we present results from characterisation, magnetic and transport properties of three different gadolinium nitride $(\mathrm{GdN})$ films and the transport properties of a samarium nitride $(\mathrm{SmN})$ film. Although $\mathrm{GdN}$ and $\mathrm{SmN}$ are the most studied materials among all members of the rare-earth nitride series, their magnetic and conducting states are still questionable. Therefore, these materials remain attractive topics for research and motivate us to further carry out research work within the experimental context. Here we report various experimental results of these materials ranging from magnetisation measurements to resistivity, Hall effect, magnetoresistance (MR) and the first thermopower measurements on rare-earth nitrides. The overall aim of is to understand the conducting nature of these materials within the existing band structure calculations and to investigate how the properties are influenced by the detailed sample preparation conditions.

This introductory chapter comprises five main sections starting with the conventional ferromagnetic semiconductors and mentioning their drawbacks which brings rare-earth nitrides (REN) to the research forefront. The second Section 1.2 covers the significant importance of RENs and the importance of rare-earth $4 f-5 d$ orbitals in magnetic and transport properties. The third section presents a general overview of rare-earth nitrides which is divided into three subsections for covering the history and fundamentals, electronic structure, and magnetic properties of rare-earth nitrides. Section 1.4 is dedicated to a literature review of GdN where primary attention is placed on reporting the different results published in the literature regarding the magnetic and electronic properties of this material. Similarly, Section 1.5 contains a literature review of $\mathrm{SmN}$, where studies performed on the magnetic and electronic properties were in particular mentioned. The last two Sections, 1.6 and 1.7, present the entire outline of this thesis and attribution, respectively. 


\subsection{Ferromagnetic semiconductors}

Spintronics is an emerging research area that exploits both the intrinsic charge of the electron and its associated spin magnetic moment [1]. Although it has been studied over the last few decades, it has recently attracted greater attention because it has the potential to develop further advances in electronic technology [1], such as increased magnetic storage capacity, reduced power consumption, and higher device speed [2]. Several research groups have already reported details of different spintronic devices, e.g., the spin diode, the spin filter and the spin transistor [2]. Scientists continue to look for new spintronics materials to enable novel or enhanced spintronic device operation.

In this regards dilute magnetic semiconductors (DMS) have been at the forefront of research because of their convincing ferromagnetic nature. DMS are the most frequently studied class of novel materials where magnetic impurity elements are added to conventional semiconductors to induce ferromagnetism [3]. Research on finding DMS materials was started in the early 1960s, which began with extensive investigation of mostly the II - VI compounds such as CdMnTe and ZnMnSe [4]. Significant research has been done from the late 1980s to find DMS materials that are ferromagnetic above room temperature. One of the first reports, published in 1989, described the growth of InAs using the molecular beam epitaxy (MBE) method where InAs doped with up to $18 \% \mathrm{Mn}$ was found to be ferromagnetic [5]. Soon after the same group reported the ferromagnetic transition in p-type InMnAs at a critical temperature of $7.5 \mathrm{~K}$ [6]. A breakthrough experiment by Ohno et al. in 1996 discovered the DMS material (Ga,Mn)As grown using the molecular beam epitaxy (MBE) method had a Curie temperature as high as $60 \mathrm{~K}$ [7]. Later on, Ohno (1998) reported that $\mathrm{Ga}_{1-\mathrm{x}} \mathrm{Mn}_{\mathrm{x}}$ As with $\mathrm{x}=5.3 \%$ was shown to have ferromagnetic character with a higher $T_{c}$ of $110 \mathrm{~K}$ [8]. Similarly, using the MBE method, T. Jungwirth et al. also found that GaAs doped with a few percent manganese (between $2 \%$ to $10 \%$ ) were shown to exhibit ferromagnetic behaviour below $170 \mathrm{~K}$ [9].

The magnetic behaviour of GaMnAs is described by various models such as the RudermanKittel-Kasuya-Yosida (RKKY) model or the Zener model [10]. The research was continued to find room temperature ferromagnetic materials to advance the cutting edge spintronics research, with reports of room temperature ferromagnetism in $\mathrm{Ga}_{\mathrm{x}} \mathrm{Mn}_{1-\mathrm{x}} \mathrm{N}, \mathrm{Ga}_{\mathrm{x}} \mathrm{Gd}_{1-\mathrm{x}} \mathrm{N}$ and transition metal doped $\mathrm{ZnO}$ made by various authors in the first decade of the twenty first century [11 - 13]. A lack of theoretical support and reproducible results make these claims 
questionable. The most crucial thing is that, the potential application of these materials are quite limited because the heavy doping of magnetic elements into the semiconductor prevents independent control of the carrier concentration [14 - 18]. Therefore the additional complexity arises on tackling the transport nature of these material push the researchers to find alternative materials.

From the above background, it can be clearly understood there is a need to consider materials that are both intrinsically semiconducting and magnetic. In principle it is then possible to independently manipulate the charge degree of freedom without affecting the magnetism, which is a key aspect for fundamental spin-transport research. Rare-earth nitrides (RENs) offer a wide range of magnetic and electronic properties. The uniqueness of controlling these two fundamental features independently ensures the significant potential application of these materials in spintronics devices $[19,20]$. Over the last few decades our understanding of these materials has improved substantially through theoretical studies as well as experimental measurements. The potential implications of these materials in advanced technology are an important motivation for studying them, but their electronic structure and magnetic properties still require more attention and need systematic investigation to provide a clearer picture which encourage us to conduct this experimental research work. 


\subsection{Importance of rare-earth $4 f-5 d$ orbitals in magnetic and transport properties}

The rare-earth elements or rare-earth metals are a set of fifteen elements in the periodic table starting from atomic number 57 to 71 . These fifteen elements are alternatively called the lanthanides [21, 22]. Scandium and yttrium are also considered rare-earth elements because of their similar chemical properties to the lanthanides. A varying electron occupation number in the highly localised $4 f$ orbital makes them interesting in terms of understanding their complex magnetotransport nature $[15,19,23-25]$.

Rare-earth elements can react with nitrogen to form rare-earth nitrides, where the RE ions have a $\mathrm{RE}^{3+}$ and $\mathrm{N}^{3-}$ configuration. In this case the number of $4 f$ electrons increases from 0 to 14 through the rare-earth series, lanthanum to lutetium [24 - 26], which therefore contribute to a different net magnetic moment for each rare-earth element depending on both the spin and orbital angular momentum of the $4 f$ electrons. The extremely localized $4 f$ levels prevent direct interaction between $f-f$ states of neighbouring $\mathrm{RE}$ atoms. Instead, the $4 f$ electrons on one $\mathrm{RE}$ ion interact with other electrons (e.g., $5 d$ ) and those electrons can interact with a neighbouring RE ion to line up with each other to show ferromagnetism. The above discussion emphasises that the $4 f$ electrons provide the magnetic moments, but they need other electrons to mediate the exchange interaction to give magnetic ordering.

The situation is a bit different when we consider the conductivity. The localised $4 f$ electrons do not directly contribute to the conductivity, rather it comes mostly from electrons in the rareearth $5 d$ orbitals that make the conduction band. These orbitals are generally not populated in ideal rare-earth nitride crystals, but electrons can be doped into them, most notably from nitrogen vacancy defects. These electrons experience and exchange interaction with the $4 f$ electrons. A consequence of that is the $5 d$ conduction band states are split into two different energy states, i.e. spin-up has a different energy to spin-down. This indicates that the conduction electrons all have only one spin orientation as long as this band is not filled with too many electrons. The important thing is that, the number of electrons in the $5 d$ level can be manipulated by varying the nitrogen vacancy concentration which therefore offers an extra degree of freedom on controlling the electronic properties of these materials without affecting the magnetic properties. 


\subsection{General overview of rare-earth nitrides}

\subsubsection{History and fundamentals of rare-earth nitrides}

Rare-earth nitrides first received attention in the early 1960s, [21, 27] when researchers were looking for new ferromagnetic semiconductors [28 - 37]. Earlier research mostly focused on bulk materials (powder samples), that were prepared by a direct reaction of the REs with $\mathrm{NH}_{3}$ or $\mathrm{N}_{2}$ gas to form the nitrides [38 - 40]. But the subsequent progress was not satisfactory due to difficulties of preparing high quality samples. Especially, most of the samples were affected by poor stoichiometry and the RENs propensity to react with oxygen in ambient conditions. Apart from the bulk samples, a significant effort was also established to grow polycrystalline and single crystal samples at that time [41, 42]. Once again, their propensity for crystal defects and lack of theoretical understanding made it difficult to identify RENs actual electronic and magnetic nature.

After more than fifty years of research, improvements in experimental techniques and a modern band structure approach has begun to provide a more reliable picture of RENs electronic and magnetic states. Especially, improvement has been made recently in film growth where more advanced techniques such as molecular beam epitaxy, sputter deposition, and pulsed laser deposition have been applied to rare-earth nitride growth [24]. As a result, nowadays around a dozen laboratories worldwide are reporting good quality samples, prepared in ultra-high vacuum environment, permitting low impurity concentrations and high surface quality [24]. Experimental studies on these recent films have corroborated the advanced band structure calculations which revealed that many of the early studies which found RENs to be semimetallic were probably due to poor stoichiometry. It is now widely accepted that many of the RENs show semiconducting nature [43 - 48] although this is still somewhat controversial [26, 49 - 52]. It is to be noted that, in the last two decades more experimental work was performed on these materials but it has been mostly limited to magnetic and conductivity studies, with a lack of attention on performing Hall effect and magnetoresistance measurements reducing the opportunity to investigate the conducting state more closely and accurately. Surprisingly, to the best of our knowledge thermopower measurements were not previously performed [53]. 
The rare-earth nitrides crystallise in the simple rock salt structure [21 - 23] as shown in Figure 1.1. Each RE atom is surrounded by six nitrogen atoms and, similarly, each nitrogen atom is surrounded by six RE atoms. A strong chemical bonding affinity is observed due to the large electronegativity difference between nitrogen (3.0) and RE (1.1-1.5) which creates robust RE$\mathrm{N}$ bonds [54]. The lattice constant, a, is defined as the distance between two neighbouring vertex atoms. For RENs it is the same distance along the $\mathrm{x}-, \mathrm{y}-$ or $\mathrm{z}-$ directions due to the symmetry of the crystal. The lattice constant typically decreases with increasing $4 f$ occupancy as expected from the decrease of cation sizes across the series with the increase of atomic number $[24,26]$.

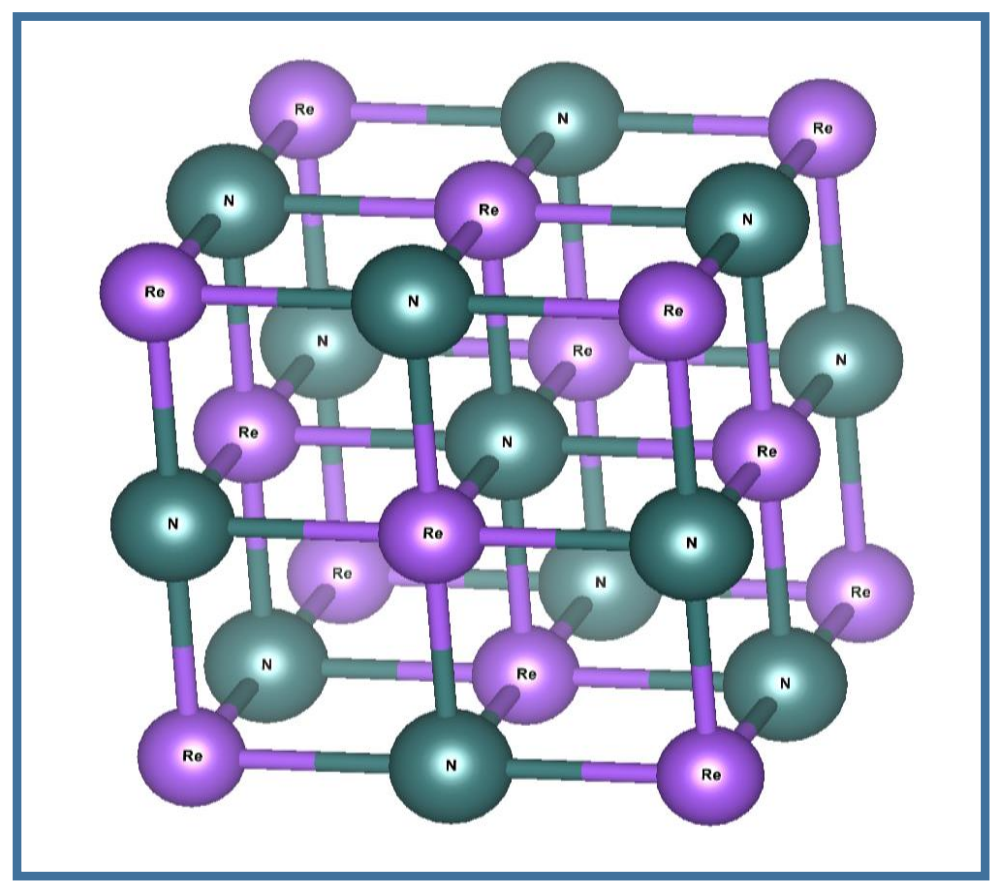

Figure 1.1: Schematic of the common rocksalt crystal structure of the RENs. The small deep lilac spheres represent the RE cations while the large cadet blue spheres are the $\mathrm{N}$ anions. 
Table 1.1 lists the experimental and calculated lattice constants of rare-earth nitrides obtained from the literature. The smallest lattice constant is reported as $4.766 \AA$ for $\mathrm{LuN}$ and it has been found to increase up to $5.305 \AA$ for $\operatorname{LaN}[24,25]$. The theoretical lattice constant of all REN series is determined from a recent first-principles calculation. The theoretical lattice constants are larger than most of the experimental lattice constants, except for $\mathrm{CeN}$ where theory predicts a 4+ oxidation state. This is appeared due to several reasons, e.g., different theoretical calculation approaches, difficult crystal structure of films and using several growth techniques $[24,25]$. Nowadays, advanced theoretical calculation predicted the lattice constant more accurately which are close to the experimentally obtained values.

Table 1.1: Experimental and calculated lattice constants of rare-earth nitrides (adapted from [24, 25]).

\begin{tabular}{|c|c|c|}
\hline REN & $\begin{array}{c}\text { Experimental lattice constant } \\
\mathbf{a}(\AA)\end{array}$ & $\begin{array}{c}\text { Calculated lattice constant } \\
\mathbf{a}(\mathbf{\AA})\end{array}$ \\
\hline $\mathrm{LaN}$ & 5.305 & 5.38 \\
\hline $\mathrm{CeN}$ & 5.022 & 4.90 \\
\hline $\mathrm{PrN}$ & 5.135 & 5.29 \\
\hline $\mathrm{NdN}$ & 5.132 & 5.24 \\
\hline $\mathrm{PmN}$ & - & 5.19 \\
\hline $\mathrm{SmN}$ & 5.035 & 5.10 \\
\hline $\mathrm{EuN}$ & 5.017 & 5.14 \\
\hline $\mathrm{GdN}$ & 4.974 & 5.08 \\
\hline $\mathrm{TbN}$ & 4.922 & 5.05 \\
\hline $\mathrm{DyN}$ & 4.895 & 5.03 \\
\hline $\mathrm{HoN}$ & 4.865 & 4.98 \\
\hline $\mathrm{ErN}$ & 4.842 & 5.00 \\
\hline $\mathrm{TmN}$ & 4.80 & 4.90 \\
\hline $\mathrm{YbN}$ & 4.781 & 4.79 \\
\hline $\mathrm{LuN}$ & 4.76 & 4.87 \\
\hline
\end{tabular}




\subsubsection{Electronic structure of rare-earth nitrides}

In this section we will discuss various theoretical approaches which have been used to predict RENs electronic state, focussing on such fundamental questions as whether RENs are metals, semiconductors or insulators in their ground state. One of the most important features of the band structure is thus to identify whether or not there is a finite band gap. Symmetry considerations of the Brillion zone indicated RENs will have the valence band (VB) maximum at $\Gamma$ and the conduction band (CB) minima at X, leading to an indirect band gap. Technically, this gap can be direct if the $\mathrm{CB}$ minimum and VB maximum occurring at the same crystal momentum $(\vec{k})$ i.e. at same $\vec{k}$ value. In contrast, one would see the indirect band gap if the $\mathrm{CB}$ minimum and VB maximum occurring at different crystal momenta which we actually observe for REN materials. In general, semiconductors are identified by having a gap in their available electronic energy levels whereas a semimetal occurs if the CB minimum and VB maximum slightly overlap in energy. The semimetal becomes a metal if the Fermi level lies well above the $\mathrm{CB}$ minimum.
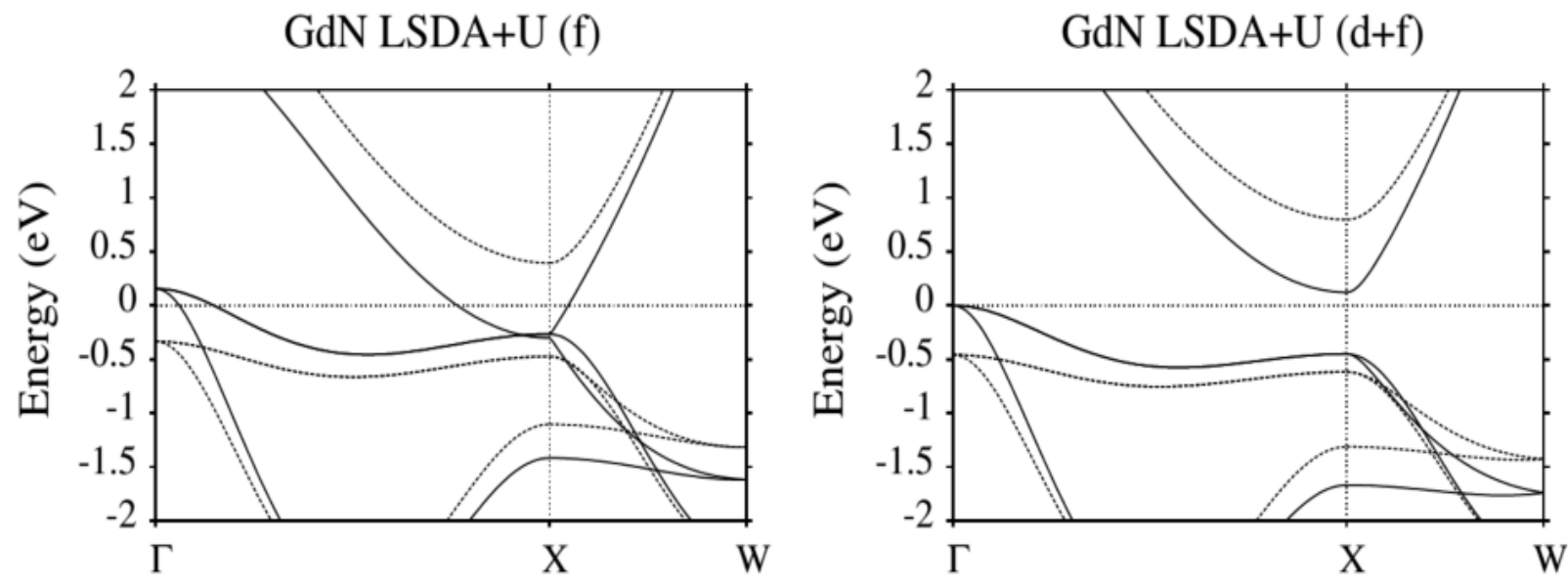

Figure 1.2: LSDA $+U$ calculation of the GdN band edges in the ferromagnetic state with $U_{f}$ (left, semimetallic) and $U_{f}+U_{d}$ (right, semiconducting) corrections applied to the $f$ - and $d$-orbitals, respectively. Solid lines: majority-spin; dashed lines minority-spin. Figure adapted from: Larson and Lambrecht [55]. 
The results of electronic band structure calculations vary significantly depending on the details of the calculation, predicting different band gaps or band overlap between the CB and VB. As an example we show the band structure of GdN in Figure 1.2 which has been adapted from Larson et al. [55]. The left side calculation, which uses a Hubbard-U correction to account for correlations among the $4 f$ electrons, produces a small overlap (semimetal) of the $\mathrm{CB}$ and VB. The right-side calculation adds an additional correction to the gadolinium $5 d$ electrons and finds $\mathrm{GdN}$ to be a semiconductor. Several computational methods have been carried out within the local spin density approximation (LSDA) to density functional theory (DFT) $[25,56]$ using a full-potential linearized muffin-tin orbital (FP-LMTO) program [25, 57]. Other theoretical calculations such as the augmented plane wave method (APW), dynamical mean field theory (DMFT), self-interaction correction (SIC), self-interactions corrected local-spin density approximation (SIC-LSDA), the GW method (G and W stand for the one-electron Green's function and screened Coulomb interaction respectively), and the local spin density approximation with Hubbard $U$ correction $(\operatorname{LSDA}+U$ ) have also been used on RENs to highlight the nature of their ground state [15, 19, 25, 26, 57 - 69].

All calculations require some level of approximations, beyond which their results are questionable to clearly predict the electronic structure of REN materials. As a result, a range of predictions such as half-metal (with an energy gap at the Fermi level in either spin-up electrons or spin-down electrons) to semiconductor were reported by these calculations $[15$, 19]. In the following paragraphs we will review some calculations and their predictions.

Considering a simple model a trivalent rare-earth ion would donate two $6 s$ electrons along with a $5 d$ or a $4 f$ electron to leave nitrogen in a -3 charge state [15]. As a result, the valence band is comprised mainly from the occupied N $2 p$ states while the conduction band forms from the empty $5 d$ and $6 s$ rare-earth states. In this scenario the $4 f$ levels remain partially occupied and treated as a strongly localised state. Taking account this electronic configuration the initial band structure calculations were performed based on the local density approximation (LDA) in density functional theory (DFT) [26]. This model considerably ignored the strong correlation among the $4 f$ electrons. In addition, the consideration of the long-ranged Coulomb repulsion between the band electrons is not fully captured by the LDA model, which results in underestimation of the band gap in semiconductors [70]. Furthermore, two different spin orientations (spin-up and spin-down) need to be treated separately; a requirement for the strong exchange interaction between the $4 f$ and the band electrons which was not accounted in this model. 
One other early calculation of the Gd and La pnictides was performed based on DFT where $4 f$ electrons were also considered as localised core electrons and tightly bound to the rare-earth nucleus. The authors performed the calculation with fine adjustment of some fitting parameters which were not known earlier. Based on this calculation, the authors predicted that the $\mathrm{CB}$ and VB overlapped in GdN and classified it as a semimetal [71]. Later advanced studies based on the Local Spin Density Approximation (LSDA) also reproduce similar results for GdN where the authors considered the strongly correlated nature of $4 f$ electrons and used the linear-muffintin orbital (LMTO) method for their calculation [72].

The first systematic attempt to calculate the band structure across the entire REN series was reported by Aerts et al. in 2004 using self-interaction corrected (SIC) local-spin-density approximation (LSDA). In this case the authors considered the localized description of the rareearth $f$ electrons along with an itinerant description of the valence electrons. According to this study, the RENs were predicted to range from metallic to insulating in their ground states [19].

After that a comprehensive study of the electronic structure of RENs was performed by P. Larson et al. [25, 55, 73]. They used the local spin density approximation to density functional theory with Hubbard $U$ corrections (LSDA $+U$ framework) to calculate the band structures of all of the RENs. In this case $U$ represents the Coulomb repulsion of electrons. The vital thing is that $U$ is made up of two components, i.e. $U_{f}$ which accounts for the localised character of the $4 f$ electrons, and $U_{d}$ which is an empirical correction of shifting the empty $d$-states up relative to the filled ones. The authors concluded that PrN, PmN, EuN and SmN are halfmetallic in nature while other RENs show small indirect band gaps that tend to increase as the rare-earth atomic number increases [25].

The most advanced technique employed to date is Hedin's quasiparticle self-consistent GW (QSGW) approach. This method automatically takes into account both $s, p, d$, and $f$ electrons in a unified single framework without applying any special treatment to the $f$ electrons. According to this method a small band gap exists for GdN in agreement with experimental results compared by the authors [61].

A review article by Duan et al. discussed all the significant theoretical progress where the relative strengths and weaknesses of all calculation methods were briefly emphasised [26]. All electronic structure calculations, magnetic properties and experimental results were summarised in the two recent review articles written by Ruck et al. and Natali et al. [15, 24]. 
It is important to mention that, there is no rigorous theoretical model has been proposed yet or accepted widely in this field; all of the models require some treatment and some approximations and so as a consequence the various models gives us slightly different results. All of these models treat their uncertainties in different ways and thus we need more experiments to validate their results.

\subsubsection{Magnetic properties of rare-earth nitrides}

Now we will discuss the magnetic properties of rare-earth nitrides, including any controversial aspects published in the literature. Interestingly, the magnetic properties of the RENs have not received as much theoretical attention as the electronic properties. In the following first two paragraphs we summarise the key results of the magnetic properties of RENs from the widely published literature whereas the next two paragraphs mainly focus on describing the instrumental parts.

The magnetic states of most rare-earth monopnictides (RE-V, where V is a group V element) have been studied since the 1960s [15, 24, 74]. Their complicated magnetic structure ranges from ferromagnetic (FM), flip-flop FM [30], to antiferromagnetic (AFM) type II. Generally, the heavier pnictides show antiferromagnetic character [32 - 34, 75 - 77], whereas almost all the nitride compounds of the lanthanides are found to be ferromagnetic at low temperature [24, 39, 41, 78 - 88]. The earlier work on the RENs mostly focused on determining their magnetic ground state and transition temperatures [15]. It is noted that due to the imperfect stoichiometry and oxygen contamination the transition temperature and exact nature of the magnetic ordering can change considerably [15]. For example, small amounts of oxygen contamination can destroy the ferromagnetic order and generate antiferromagnetic correlations in $\operatorname{GdN}[89,90]$. In addition it has been found that nitrogen vacancies can change the magnetic ordering temperatures in rare-earth nitrides [91 - 93].

Around a decade ago, the magnetic properties of RENs were re-investigated with the combined efforts of advanced band structure calculation techniques and the growth of high-quality thin films. The advanced band structure calculations may help to explain the key question of what causes the exchange mechanism in the RENs, particularly in GdN [81, 94 - 96], with several exchange mechanisms proposed to explain the magnetic state of RENs [24, 26, 96], including 
a magnetic polaron concept considered to explain differences in observed transition temperatures [97].

Experimental investigations have utilised both direct magnetisation measurement techniques (e.g., super conducting quantum interference device (SQUID), vibrating sample magnetometer (VSM)) and the element specific technique $\mathrm{x}$-ray magnetic circular dichorism (XMCD) which can provide information about the spin and orbital contributions to the magnetism individually [98 - 102]. XMCD is especially useful for probing materials like $\mathrm{SmN}$ that have a very small magnetization, and also REN alloys and superlattices where the contribution of the magnetization from each element can be evaluated precisely. However, the magnetic state of RENs is still debatable [15, $24-26,103]$ and needs further systematic theoretical and experimental investigations to provide a clear picture.

Until now we have gained the general understanding of rare-earth nitrides including their structural, electronic and magnetic properties without focussing on any particular REN material. Now we are at the stage to continue more focused discussion on selected rare-earth nitrides, i.e. GdN and $\mathrm{SmN}$, because they are the rare-earth nitrides which are studied during this Ph.D. project.

\subsection{Literature review of gadolinium nitride}

Gadolinium nitride $(\mathrm{GdN})$ is the most theoretically and experimentally studied material among the rare-earth nitride (REN) series [15, 24 - 26, 44, 69, 86, 93 - 105]. Similar to all other members of the rare-earth nitride series, GdN exhibits a rocksalt structure with a bulk lattice parameter often quoted to be $4.98 \AA$, and it shows strongly localised $4 f$ states. The partial charge distribution of $\mathrm{Gd}$ and $\mathrm{N}$ is close to $3^{+}$and $3^{-}$respectively, which plays a significant role in controlling the magnetic and transport properties of $\mathrm{GdN}$. Gadolinium (Gd) is located in the middle of the rare-earth group in the periodic table and has a half-filled $4 f$ shell with 7 unpaired electrons. The exactly half-filled $4 f$ shell electrons simplifies theoretical calculations and has attracted the attention of researchers since research began in this field [15, 24 - 26]. Recent research has focused on producing devices using electronic spin as well as the charge degree of freedom, where understanding the magnetic and conduction nature of $\mathrm{GdN}$ is the priority for researchers $[15,24]$. 
Interestingly, GdN has the same crystal structure as one of the first discovered ferromagnetic semiconductor materials $\mathrm{EuO}[15,24,86,106]$ and both materials have an identical ${ }^{8} \mathrm{~S}_{7 / 2} 4 f$ shell configuration. Furthermore, both compounds have almost the same transition temperature and zero orbital angular momentum in the $4 f$ shell $[15,19,24,86]$. The above discussion makes clear that both $\mathrm{EuO}$ and GdN have very similar predicted and observed properties, although $\mathrm{EuO}$ has received more attention than GdN.

The magnetic moment/unit volume of GdN arises mostly from the $4 f$ electrons. The electronic configuration of the $f$ orbital of $\mathrm{Gd}^{3+}$ ions is $4 f^{7}$ that gives an exactly half-filled $f$ orbital leading to a total orbital angular momentum $\mathrm{L}=0$ and a spin angular momentum $\mathrm{S}=\frac{7}{2}$. The total angular momentum of $\mathrm{Gd}^{3+}$ is thus $\mathrm{J}=\frac{7}{2}$, and the expected value of the total magnetic moment is $\mathrm{M}=7 \mu_{\mathrm{B}}$. The earliest investigation claimed that $\mathrm{GdN}$ is ferromagnetic with a transition temperature $\left(T_{c}\right) 70 \mathrm{~K}$ and a magnetic moment $7 \mu_{\mathrm{B}} / \mathrm{Gd}^{3+}$ atom at $4.2 \mathrm{~K}[37,79,81]$. In contrast, a great variation in the transition temperature and the magnetic moment from one sample to another has been reported by other researchers [15, 24, 44, 69, 107 - 110]. In this regard, a significant study was done by Leuenberger et al. in 2005 and they reported that GdN shows ferromagnetic ordering below $60 \mathrm{~K}$ [86]. A theoretical study by Larson et al. revealed that GdN showed a ferromagnetic nature but with a much lower predicted Curie temperature [55].

But the debate continues as further studies on stoichiometric polycrystalline $\mathrm{GdN}$ with a lattice constant of $4.974 \AA$ have shown it to have a transition temperature of $58 \mathrm{~K}$ [41, 85], which is lower than the other reported values of $65 \mathrm{~K}$ or $70 \mathrm{~K}$. In this case, a lower saturation magnetic moment $\left(6.84 \mu \mathrm{B} / \mathrm{Gd}^{3+}\right)$ was obtained as compared to the commonly measured value of 7.0 $\mu \mathrm{B} / \mathrm{Gd}^{3+}$. Research lead by our group demonstrated that, the value of $T_{c}$ is influenced by nitrogen vacancies and they reported the transition temperature of non- stoichiometric nitrogen deficient polycrystalline GdN films can be enhanced up to $200 \mathrm{~K}$ [93]. In contrast, Yoshitomi et al. reported that the lowest transition temperature of $\mathrm{GdN}$ varied between 20 and $37 \mathrm{~K}$ [91, 111 - 113]. Over the last few years, systematic investigation has been done to establish a link between the magnetic behaviour and the nitrogen vacancy $\left(V_{N}\right)$ concentration, and this remains an active field of research.

The conducting nature of $\mathrm{GdN}$ is more debatable than its magnetic nature. Band structure calculations are helpful to predict the conducting state of a material. This material has been studied since 1960 but several research groups have proposed different theoretical methods to obtain the conducting state of this material. Aerts et al. predicted that GdN was half-metallic 
by using density functional theory in the local spin density approximation with a selfinteraction correction [19], whereas Larson et al. reported $\mathrm{GdN}$ was a semiconducting material according to their local spin density approximation plus the Hubbard U correction (LSDA $+U$ ) method [25]. Further study by Duan et al. found a half - metallic ground state for GdN from the LSDA $+U$ calculation $[26,52]$. Ghosh et al. observed that a small variation in the starting calculation parameters could cause the conduction band to cross the Fermi level, and they concluded that the conducting state of GdN lies somewhere between the metal and the semiconductor regions, possibly undergoing a metal-insulator transition at the transition temperature [60]. Note that, all these predictions emphasise that they are quite uncertain as tweaking some parameters within their calculations can significantly alter the results.

Experiments are needed to test the theoretical predictions. Leuenberger et al. revealed that resistivity data of GdN showed semiconducting behaviour above the transition temperature [86]. In contrast, when the temperature dropped below the transition temperature, the resistivity of GdN decreased with decreasing temperature. They interpreted this phenomenon by the exchange splitting of the conduction band; at low temperature the conduction band crossed below an impurity level due to an exchange splitting and as a result more carriers were available in the conduction band [86]. A later resistivity experiment carried out by Granville et al. found $\mathrm{GdN}$ was a semiconductor even below the transition temperature and their finding supports the exchange splitting interpretation [44]. A recent experimental result published by Natali et al. also has seen the semiconducting nature of this material [97]. Various experimental results showed that both nitrogen vacancies and oxygen impurities can significantly affect the conductivity of $\mathrm{GdN}[44,93,109,114]$. A complete understanding of the conducting nature is yet to be formulated and requires more systematic investigation.

The optical properties of GdN have taken the attention of several research groups. It is important to note that optical absorption measurements generally give the direct gap of a material; a material can still be semimetallic with an overlap of the valence and conduction bands even if the measured gap is greater than zero. Schneemeyer et al. reported an energy gap of a GdN single crystal greater than $1.5 \mathrm{eV}$ through their transmittance measurement [83] while theoretical calculations reported contradicting values of the band gap of $1.1 \mathrm{eV}-1.2 \mathrm{eV}$ [115], $0.98 \mathrm{eV}[55,106]$ and $5.0 \mathrm{eV}$ [95]. The optical energy gap on a $200 \mathrm{~nm}$ thick polycrystalline GdN film was first systematically investigated and presented by Trodahl et al. [116]. The authors reported that a minimum direct majority-spin gap of $0.91 \mathrm{eV}$ was observed in the ferromagnetic state. The paramagnetic state shows an average optical gap of $1.30 \mathrm{eV}$. A red 
shift of the optical band gap was observed in the ferromagnetic state which was attributed to a narrowing of the band gap caused by exchange splitting of the conduction and valence bands. Figure 1.3 presents a calculated band structure of GdN performed using the LSDA $+U$ method where the value of $\mathrm{U}_{d}$ has been chosen to obtain agreement between theory and the optically measured band gap. The solid red lines represent the majority-spin bands (those with the same spin orientation and the occupied $4 f$ orbitals) and the blue dashed lines represent the minorityspin. The Figure 1.3 shows that the valence band is composed of $\mathrm{N} 2 p$ levels whereas the conduction band arises mainly from the $5 d$ levels of $\mathrm{Gd}$. The narrow band above $4.0 \mathrm{eV}$ represents the unoccupied $4 f$ levels and the occupied $4 f$ levels lie at about - $8 \mathrm{eV}[94,116,117]$.

Recent optical studies on polycrystalline GdN samples show an optical gap of $1.3 \pm 0.05 \mathrm{eV}$ and claim that GdN is a ferromagnetic semiconductor [92], which is nearly in agreement with Trodahl et al. [116]. However, both authors have reported different transition temperatures for their samples $[92,116]$. Yoshitomi et al. also reported a band gap of $0.95 \mathrm{eV}-1.18 \mathrm{eV}$ of epitaxial GdN films [91, 111].

It is now clear that various research groups have reported different electronic, magnetic and optical results of $\mathrm{GdN}$ which thus requires more systematic investigation to reach an agreement. This thesis has investigated the transport properties of this material within the experimental context with the special focus on resistivity as a function of temperature and field, Hall effect and thermopower experiments. 


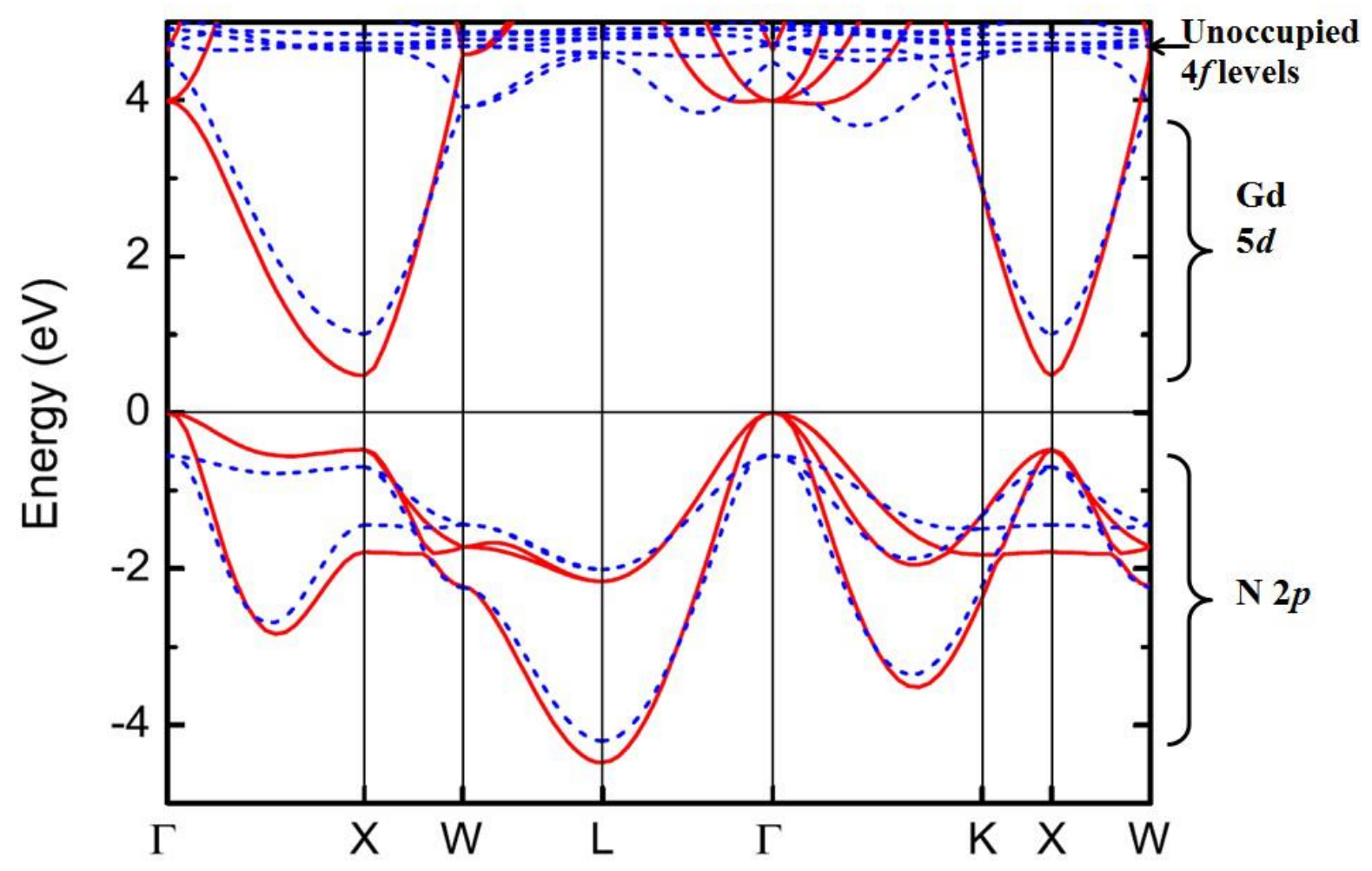

Figure 1.3: The calculated band structure of GdN (adapted from [116]).

The solid lines represent majority-spin bands, and dashed lines the minority-spins. 


\subsection{Magnetic and electronic properties of samarium nitride}

SmN has not had as much attention as GdN but significantly more research work has been done on this material as compared to other member of RENs [24]. Despite having the same crystal structure as GdN with very close lattice constants (5.03 $\mathrm{A}), \mathrm{SmN}$ shows different magnetic and electronic properties. The trivalent samarium ion $\left(\mathrm{Sm}^{3+}\right)$ has a $4 f^{5}$ configuration, which is two electrons less than a half-filled $4 f$ orbital. Hund's rules yield a spin angular momentum $S=5 / 2$ and a total orbital angular momentum $\mathrm{L}=5$. In the ground state these two momenta oppose each other, resulting in a total angular momentum quantum number $\mathrm{J}=|\mathrm{L}-\mathrm{S}|=5 / 2$. The ferromagnetically aligned spin magnetic moment and the orbital magnetic moment are equal in magnitude with opposite sign; therefore they almost cancel each other and contribute a nearly zero total magnetic moment.

Due to the very small moment the earlier experiments on bulk $\mathrm{SmN}$ were interpreted as antiferromagnetic ordering instead of ferromagnetic [24, 35]. The early research reported the Neel temperature $T_{N}$ was $13 \mathrm{~K}$ [118], $15 \mathrm{~K}$ [119], or $18.2 \mathrm{~K}$ [120]. The antiferromagnetic nature of this material was rejected later once the subsequent measurements of both neutron scattering [121] and recent XMCD studies $[122,123]$ very clearly showed the ferromagnetic nature with a small magnetic moment.

The recent experiments on $\mathrm{SmN}$ thin films show the ferromagnetic transition below $30 \mathrm{~K}$ [20, 124]. In its ferromagnetic state, the magnetic hysteresis loop exhibits a large coercive field when the temperature is decreased to around $2 \mathrm{~K}$, while a very small saturation magnetic moment of around $0.035 \mu_{\mathrm{B}} / \mathrm{Sm}^{3+}$ remains at this temperature. In addition, Larson et al. calculated the band structure of SmN, using the LSDA $+U$ method and predicted nearly zero moment, consistent with SQUID measurements [25]. The XMCD based studies on SmN demonstrated that the $4 f$ spins align ferromagnetically but the contribution of moment from $4 f$ orbital effectively cancels the resulting spin moment [122].

The transport properties of $\mathrm{SmN}$ were also studied by various researchers. Initially a semimetallic behaviour of SmN was reported by Didchenko and Gortsema [124], while later on SmN was suggested to be a semiconductor with a band gap of $0.7 \mathrm{eV}$ [21]. An experimental study by Preston et al. reported that $\mathrm{SmN}$ shows semiconducting behaviour [20]. Similarly, Meyer et al. also reported that the $\mathrm{SmN}$ is found to be semiconducting in its ground state [125]. 
In sharp contrast to that a current electronic structure calculation based on the LSDA $+U$ method favours a half-metallic ground state [120]. The latest research by our group reported that degenerately doped $\mathrm{SmN}$ shows a superconducting nature below $4 \mathrm{~K}$ [126]. Various theoretical calculations and experiments were performed to understand the electronic band structure of SmN but some fundamental questions need to be answered clearly which encourage us to further study the transport properties of this material.

\subsection{Synopsis}

This thesis is comprised of nine chapters, this introduction being the first where we highlighted the important aspects of ongoing research on REN materials. The following descriptions provide the key themes of the rest of the chapters.

\section{Chapter 2: Fundamentals of magnetism and transport properties}

This chapter highlights the necessary concepts and theories that have been used to interpret the results reported in this thesis. This chapter begins with the basic understanding of magnetism followed by electrical transport mechanisms. The transport part of this chapter is categorised in two sections. The first part covers the classification of materials according to their transport nature, a simple discussion of the Drude model to explain the resistivity and magnetoresistance of a material, and the theory of the Hall effect and the extraordinary Hall effect. After this the chapter focusses on the theory of thermoelectric properties and its various aspects.

\section{Chapter 3: Sample preparation and characterisation techniques}

This chapter contains two major sections: sample preparation techniques and characterisation results. In the first section we discuss the sample preparation technique using thin film growth in an ultra-high vacuum chamber (UHV). All parameters related to sample growth are discussed briefly followed by the structure of growth chambers and the growth process. The second section presents the basic theory for the X-ray diffraction and Rutherford backscattering spectrometry techniques used for structural and compositional characterisation. 


\section{Chapter 4: Experimental techniques}

This chapter explains the techniques and methodologies used for magnetic and transport measurements. A superconducting quantum interference device (SQUID) magnetometer is used to perform the magnetic measurements. A cryostat and a Quantum Design physical property measurement system (PPMS) are used to perform temperature dependent resistivity, magnetoresistance and Hall effect measurements. The room temperature Hall effect measurement is performed using an Ecopia HMS-3000 system at the GNS science, Lower Hutt. A thermopower set-up has been developed to conduct the thermopower measurements of rareearth nitride films. A detailed discussion is therefore carried out highlighting the thermopower rig and measurement process.

\section{Chapter 5: Magnetotransport properties of high carrier concentration epitaxial gadolinium nitride film (GdN_HC)}

This is the beginning chapter of the experimental results. In this thesis we present data from three different GdN films grown under different conditions. Two of the GdN samples are epitaxial films grown at higher temperature in Valbonne, France, while the other GdN film is polycrystalline, grown at room temperature in the UHV lab at Victoria University of Wellington, New Zealand. The two epitaxial films contain different carrier concentrations and we named them GdN_HC and GdN_LC, where HC and LC stand for high carrier concentration and low carrier concentration, respectively.

This chapter presents the magnetotransport results of the GdN_HC film. The first section of this chapter is based on the magnetic results which reports the ferromagnetic transition temperature, saturation magnetisation, and coercive fields. We will correlate the magnetic results of this chapter with the following transport results to understand the conducting nature of this film.

In the second section we report the temperature dependent resistivity, Hall effect measurements, magnetoresistance measurements and thermopower measurements of this epitaxial GdN film. The results imply that the transport is dominated by electrons moving in a highly delocalised conduction band. The behaviour is interpreted in terms of a model including a resonant impurity state lying above the conduction band minimum. 


\section{Chapter 6: Magnetotransport properties of low carrier concentration epitaxial gadolinium nitride film (GdN_LC)}

In this chapter we discuss the magnetic and transport properties of the low carrier concentration epitaxial GdN film. The major discussion in this chapter is based on the temperature dependent resistivity and Hall effect results. A phenomenological model based on the Brillouin function is used for the first time to interpret the Hall effect results in the paramagnetic region while the experimental magnetic results are used to fit the extraordinary Hall effect data in the ferromagnetic region.

\section{Chapter 7: Magnetotransport properties of polycrystalline gadolinium nitride film}

In this chapter we discuss the magnetic and transport properties of a polycrystalline GdN film. The first section focusses on the magnetic results which are interpreted based on the existing magnetic polaron scenario. The second section presents an in-depth discussion of the temperature dependent resistivity, the room temperature Hall effect and the magnetoresistance of this film. In particular, in this film we see the highest reduction of resistance in the presence of a magnetic field seen in any GdN film.

\section{Chapter 8: Magnetotransport properties of SmN film}

This chapter presents the transport properties of an epitaxial $\mathrm{SmN}$ film grown in Valbonne, France. We present temperature dependent resistivity, Hall effect, magnetoresistance and thermopower results of this film. Similar to the epitaxial GdN film, the thermopower measurement was performed for the first time on $\mathrm{SmN}$ film, with the results from the two different RENs showing similar behaviour. 


\section{Chapter 9: Discussion and future possibilities}

In the final chapter, we summarise all results of each film discussed in this thesis. We propose studies to further elucidate the transport properties of rare-earth nitrides. Especially, thorough investigation of the thermopower will be especially useful to explore more interesting transport results of rare-earth nitride films. In addition, temperature dependent resistivity, Hall effect and magnetoresistance measurements of $\mathrm{GdN}$ and $\mathrm{SmN}$ equally require more attention to clearly answer all fundamental questions and enhance their application in cutting edge research on spintronics. 


\subsection{Attribution}

Generally, a $\mathrm{PhD}$ thesis requires the contributions from many people, and my thesis is no exception. Many of the experiments were performed by collaborating with many research groups in Wellington, New Zealand. Their contributions are highlighted as follows,

Film growth: I performed polycrystalline $\mathrm{GdN}$ and $\mathrm{SmN}$ film deposition assisted by F. Natali and H. Warring. Two epitaxial GdN films and one epitaxial SmN film were obtained from our collaborator in Valbonne, France.

X-ray diffraction: Performed by me with initial help from B. Ruck.

RBS: Conducted by P. Murmu and J. Kennedy at the GNS Science.

Magnetic measurement: I was responsible for the magnetic measurements which were conducted at Robinson Research Institute (RRI). I carried out all the measurements and data analysis.

Resistivity measurement: I performed the resistivity measurement in the physical property measurement systems (PPMS) from Quantum Design PPMS.

Hall effect and magnetoresistance measurements: I was responsible for these two measurements conducted at Robinson Research Institute (RRI). Initially I was assisted by S. Chong and S. Granville with sample mounting process and running the sequence file in a physical property measurement system (PPMS), until I was familiar with the complete process.

Room temperature Hall effect measurement: I performed the room temperature Hall effect measurement for the desire samples at the GNS Science.

Thermopower measurement: I was mainly responsible for running this experiment at VUW, Wellington. J. Trodahl and B. Ruck helped me to design and develop the thermopower rig. All thermopower experiments were conducted by myself with initial assistance from J. Trodahl and B. Ruck. 


\subsection{Publications}

\section{Published/Submitted:}

1. T. Maity, H. J. Trodahl, F. Natali, S. Vézian and B. J. Ruck. "Electron Transport in Heavily Doped GdN”, Physical Review Materials 2, 014405 (2018).

2. J. Kennedy, P. Murmu, J. Leveneur, G. V. Williams, G. V, R. L. Moody, T. Maity and S. V. Chong. "Enhanced Power Factor and Increased Conductivity of Aluminum Doped Zinc Oxide Thin Films for Thermoelectric Applications”, Journal of Nanoscience and Nanotechnology, Vol. 18: 1384-1387, (2018).

3. T. Maity, H. J. Trodahl, S. Granville, S. Vézian, F. Natali and B. J. Ruck. “Magnetoresistance of GdN”, - PACS numbers: 72.25.Dc, 73.50. (submitted - 2019).

4. T. Maity, H. J. Trodahl, B. J. Ruck and S. Granville. "Anisotropic Magnetoresistance of GdN”, - PACS numbers: 72.25.Dc, 73.50.Jt 75.50.Pp. (submitted - 2019).

\section{In-Progress:}

5. T. Maity, H. J. Trodahl, S. Granville, S. Vézian, F. Natali and B. J. Ruck. “Magnetotransport Properties of SmN”, - PACS numbers: 72.25.Dc, 73.50.Jt 75.50.Pp.

6. T. Maity, H. J. Trodahl, L. Figueras and B. J. Ruck. "Magnetotransport properties of (Gd,Sm)N alloy films”, - PACS numbers: 72.25.-b 72.25.Dc, 73.50.Jt 75.50.Pp.

7. T. Maity, H. J. Trodahl and B. J. Ruck. "Large Magnetoresistance of Ferromagnetic GdN films”, - PACS numbers: 72.25.Dc, 73.50.Jt 75.50.Pp.

8. T. Maity, H. J. Trodahl, S. Vézian, F. Natali and B. J. Ruck. "Magnetotransport properties of (GdN/SmN) Superlattice”, - PACS numbers: 72.25.Dc, 73.50.Jt 75.50.Pp. 


\section{Chapter 2}

\section{Fundamentals: Magnetism and Transport}

\subsection{Introduction}

This chapter outlines fundamental theories of magnetism and transport properties of materials. Note that, due to the nature of the experimental thesis we discuss theories relevant to interpret our results within the experimental context rather than a theoretical calculation perspective. The chapter is categorised in two main sections. In the magnetism section, we discuss the magnetism in rare-earth nitrides, Hund's rules, and fundamental differences between diamagnetism, paramagnetism and ferromagnetism. A discussion on ferromagnetism and the exchange interaction takes the primary attention in this section.

The transport part starts by defining solids in three major categories, i.e. metal, semiconductor and insulator. The next discussion in this section is based on a classical Drude model of metallic conduction using the simple textbook expression followed by the Hall effect and extraordinary Hall effect. Section 2.2.6 contains details about magnetoresistance. The final Section 2.3 deals with a definition and the physical origin of thermopower followed by specific details of the diffusion thermopower which has been used to interpret our data in the results chapters (Chapter 5 and Chapter 8). 


\subsubsection{Fundamentals of magnetism}

The origin of magnetism in materials is related to the quantum mechanics of many-body systems, while the experimentally measurable magnetisation is governed by crystal structure, shape, growth conditions and quality of the material. Electrons have both spin and orbital angular momentum and the net magnetisation comes from both contributions. Using Hund's rules we can estimate the spin and orbital contributions separately.

The orbital angular momentum quantum number of a single electron is $l$ and the eigenvalue is $\hbar \sqrt{l(l+1)}$. The spin angular momentum quantum number of a single electron is $s$ and the eigenvalue is $\hbar \sqrt{s(s+1)}$, where, ' $s$ ' is always equal to $1 / 2$; either in spin-up or spin-down state. Within an atomic shell there are $(2 l+1)$ levels or orbital states, and a total of $(2 s+1=$ 2) possible spin states (spin-up and spin-down) are available for each orbital state. These orbitals are labelled by the orbital magnetic quantum number, $m_{l}$ and spin magnetic quantum number $m_{s}$, respectively, which are allowed to take integer values from $-l$ to $+l$ or $-s$ to $+s$. The total number of states in a shell is thus equal to $2(2 l+1)$. For example, the $4 f$ shell in rare-earth nitrides, with $l=3$, can have $m_{l}$ values of $-3,-2,-1,0,1,2,3$, and up to 14 electrons can be accommodated in this shell $[127,128]$. Thus, the total angular momentum $\vec{J}=$ $\vec{L}+\vec{S}$ and has magnitude $\hbar \sqrt{j(j+1)}$, where upper case letters $S$ and $L$ are used to denote the net spin angular momentum quantum number and net orbital angular momentum quantum number for all electrons in a particular material. The quantum number $J$ can range from $\mid L+$ $S \mid$ to $|L-S|$ in integer steps.

If a system contains more electrons, then calculating the total magnetic moment will be more complicated. It is important to note here that, for a filled shell of electrons, $\vec{L}, \vec{S}$, and $\vec{J}$ are equal to zero, so there is no net angular momentum which can contribute to the magnetic dipole moment. For example, noble gas atoms have no incomplete shells, thus a permanent dipole moment cannot arise and this type of atom is classified as a diamagnet [127]. Therefore, we only have to consider atoms that contain incomplete or partially filled outer shells of electrons in calculating $J, L$, and $S$. The rare-earth nitrides have partially filled $4 f$ shells which therefore contributes different $J, L$, and $S$ values. As a result the net magnetic moment per unit volume changes across the REN series and makes these materials interesting in potential spintronics applications. 
The importance of the $4 f$ shell in RENs magnetism is already mentioned in Chapter 1 and how the $4 f$ electrons contribute to the magnetism through the exchange interaction is mentioned later in section 2.1.6.

\subsubsection{Hund's rules}

To determine the value of $L$ and $S$ of an individual atom (RE atom in our case), one has to consider Hund's rules. The German physicist Friedrich Hund suggested a series of three empirical rules to identify the lowest-energy configuration for the electrons in a partially filled shell (the rules do not predict the actual energy) [127, 129]. Hund's first rule states that the electrons maximise their total spin $S$. This means that the electrons will firstly occupy orbitals with one electron per orbital and align their spins parallel to each other until all the orbitals contain one electron each. After that, the electrons are forced to "pair up" in orbitals, in pairs of opposite spin consistent with the Pauli exclusion principle. This is because electrons with the same spin are required (by the Pauli exclusion principle) to avoid each other to minimise the energy.

Hund's second rule states that, for a given spin arrangement, energy is minimised by choosing the maximum value for $L$ consistent with the first rule. The meaning of this rule is that, orbitals are populated starting with the highest possible $m_{L}$ values. The final rule is due to the spinorbit coupling and relates $L$ and $S$ to the total angular moment. This rule states that $J$ is minimised if the shell is less than half full $(J=L-S)$, while $J$ is maximised for more than half full shells $(J=L+S)$.

Hund's rules are most applicable to rare-earth elements, which have localised $4 f$ electrons, unlike transition elements which have delocalised $3 d$ electrons. Larson et al. performed the theoretical treatment to estimate the total magnetic moment of several rare-earth nitrides as shown in Figure 2.1 [25]. The Hund's rule solution yields the orbital and spin components of the magnetic moment and thus the total magnetic moment for each rare-earth nitride material. The total magnetic moment is estimated using $\mu_{B}(L+2 S)$, where $\mu_{B}$ is the Bohr magneton. The factor of 2 arises because of the $g$-factor of the electron, which is approximately 2 for spin angular momentum and 1 for orbital angular momentum. The figure shows that lighter rareearth nitrides ( $\mathrm{PrN}-\mathrm{EuN})$ have the total orbital moment opposite to the spin moment whereas 
in the heavier rare-earth nitrides ( $\mathrm{DyN}-\mathrm{YbN}$ ) the orbital moment is parallel to the spin moment. This essentially arises because of Hund's third rule. Note that, this is a calculation and may not be correct for many of RENs.

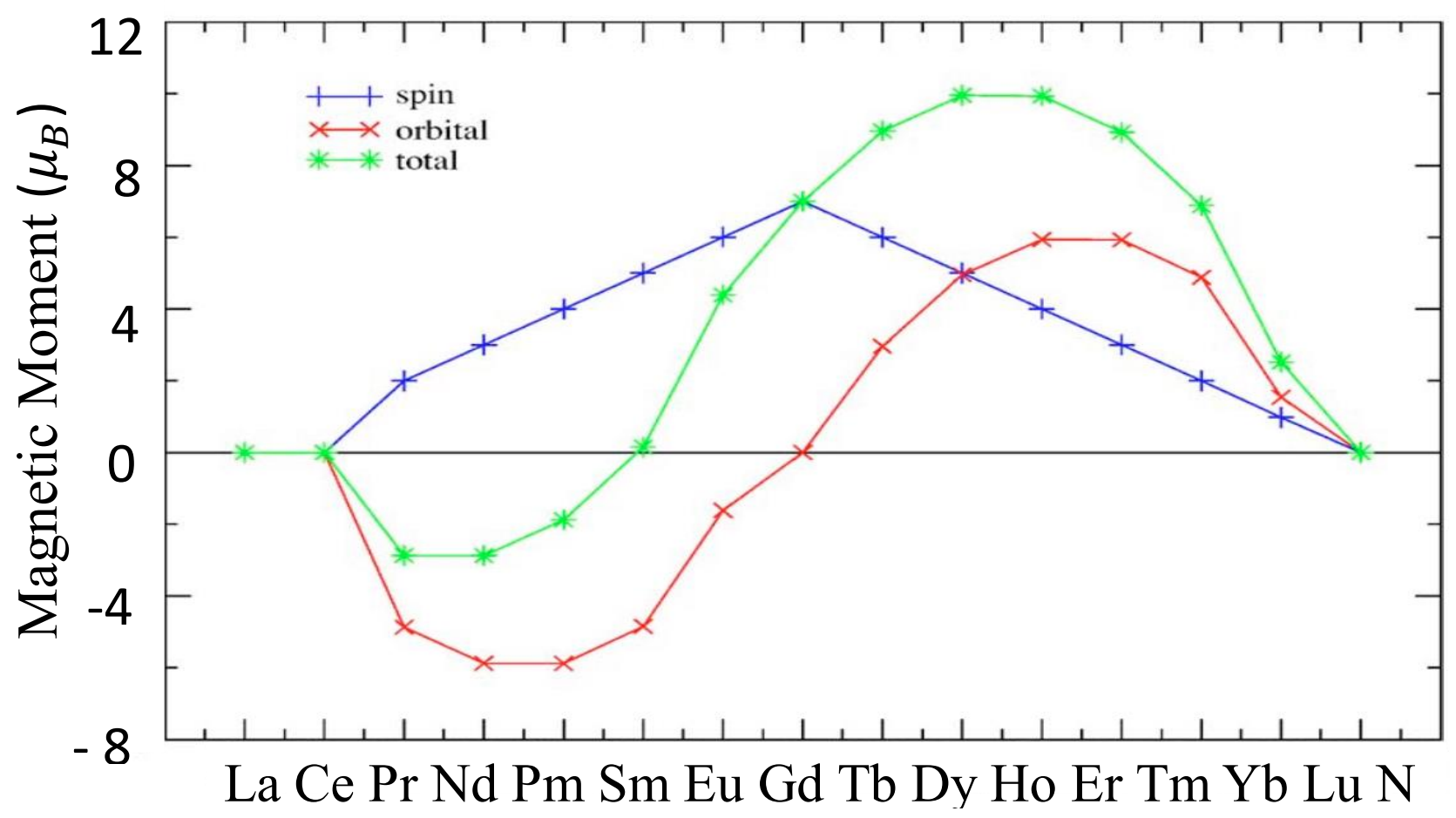

Figure 2.1: Total magnetic moment of rare-earth nitride series. The orbital and spin components of the magnetic moment are indicated by red and blue colours, respectively. This figure is reproduced from Larson et al. [25). 


\subsubsection{Magnetic susceptibility}

The magnetic behaviour of materials can be classified according to their response to an external applied field. The magnetisation $\mathrm{M}$ of a material is defined by the magnetic moment per unit volume and the relation between magnetisation and external field is the magnetic susceptibility, which is given by

$$
\chi=\frac{M}{H}
$$

where $H$ represents the strength of the external magnetic field. Many materials exhibit a spontaneous magnetisation with zero applied field. Therefore, in this particular scenario, Equation 2.1 is less useful. According to the value of magnetic susceptibility, materials can be categorised as diamagnetic, paramagnetic, ferromagnetic or antiferromagnetic.

\subsubsection{Diamagnetism and Paramagnetism}

In a diamagnetic material the magnetic susceptibility is small with weak temperature dependence. The induced magnetic response of a diamagnetic material always occurs in the opposite direction to the applied field, the diamagnetic susceptibility is negative and typically has a value of $\chi \approx-10^{-5}$. Paramagnetism is observed generally in materials with atoms of partially filled electronic shells (e.g., $\mathrm{Fe}^{2+}, \mathrm{Gd}^{3+}$ ). In the case of paramagnetism, the magnetic moments have a tendency to turn towards the direction of the applied field. If no opposing force acts, complete alignment of the atomic moments will occur and the material will show a very large magnetisation in the direction of the applied field. However, thermal agitation of the individual moments counteracts this tendency and tries to keep the magnetic moments at random. The result is only a partial alignment in the field direction. Therefore, a weak magnetisation and a small positive susceptibility appear. Typically, the susceptibility $(\chi)$ of paramagnetic materials ranges between $10^{-3}$ to $10^{-5}$ and depends on temperature $[127,128,130$ $-135]$. 
The magnetisation of a collection of non-interacting magnetic moments as a function of temperature and magnetic field strength is

$$
\mathrm{M}=\mathrm{N} \frac{\sum_{-J}^{+J} M_{J} g \mu_{B} \exp \left(M_{J} g \mu_{B} H / k_{B} T\right)}{\sum_{-J}^{+J} \exp \left(M_{J} g \mu_{B} H / k_{B} T\right)}
$$

where, $\mathrm{N}: \quad$ The number of magnetic ions / unit volume

$\mathrm{J}$ : The total angular momentum quantum number

$M_{J}: \quad$ Total magnetic moment and it values ranges from $\mathrm{J}, \mathrm{J}-1, \mathrm{~J}-2, \ldots . \mathrm{J}+2,-\mathrm{J}+1,-\mathrm{J}$

$k_{B}: \quad$ Boltzmann constant $=1.38 \times 10^{-23} \mathrm{JK}^{-1}$

$g$ : Landé $g$ - factor, defined in terms of the spin, orbital, and total angular momentum quantum numbers. It can be written as

$$
g=1+\frac{J(J+1)+S(S+1)-L(L+1)}{2 J(J+1)}
$$

When the field strength is smaller than the thermal energy, that is $g \mu_{B} H<<k_{B} T$, the above equation reduces to

$$
\mathrm{M}=\frac{N g^{2} J(J+1) \mu_{B}^{2} \mathrm{H}}{3 k_{B} T}
$$

Therefore, the susceptibility $\chi$ for a collection of non-interacting atoms or ions is inversely proportional to the temperature, leading to the well-known result of Curie's law,

$$
\chi=\frac{N \mu_{B}^{2} P_{e f f}^{2}}{3 k_{B} T}
$$

where, $\mathrm{P}_{\text {eff }}=\mathrm{g} \sqrt{J(J+1)}$ is the effective Bohr magneton number.

The Curie constant, $\mathrm{C}$, is defined as

$$
\mathrm{C}=\frac{N \mu_{B}^{2} P_{e f f}^{2}}{3 k_{B}}
$$


However, Curie's law (Equation 2.5) is not valid at low temperatures or larger fields. Therefore, at low temperature or larger field strengths $\left(g \mu_{B} H<k_{B} T\right)$, the magnetisation $\mathrm{M}$, of $\mathrm{N}$ non-interacting ions in volume $\mathrm{V}$ at temperature $\mathrm{T}$ becomes, from Equation 2.2 [132]:

$$
M=\frac{N}{V} g J \mu_{B} B_{J}(x)
$$

where, $x=\frac{g J \mu_{B} H}{k_{B} T}$, and $B_{J}(x)$ is the Brillouin function which is defined as

$$
B_{J}(x)=\frac{2 J+1}{2 J} \operatorname{coth} \frac{2 J+1}{2 J} x-\frac{1}{2 J} \operatorname{coth} \frac{1}{2 J} x
$$

When $T \rightarrow 0$, Equation 2.7 shows a finite magnetisation for a finite number of magnetic atoms. For a semi-classical free particle, where $J \rightarrow \infty$, the $B_{J}(x)$ reduces to

$$
B_{J}(x)=\operatorname{coth} x-\frac{1}{x}=L(x)
$$

where, $L(x)$ is known as the Langevin function.

\subsubsection{Ferromagnetism}

Many materials (e.g., Fe, Co, Ni, Gd) exhibit a spontaneous magnetisation at low temperatures, even in the absence of an external magnetic field. These materials are known as ferromagnets. In a ferromagnetic material, the atomic magnetic moments tend to align parallel to each other and the material shows large positive $\chi$ values, ranging from 50 to 10,000 . The spontaneous magnetisation of a ferromagnetic material can occur only below a certain temperature $T_{c}$ called the ferromagnetic Curie temperature. Above the Curie temperature $\left(T_{c}\right)$, the material typically exhibits a paramagnetic characteristic. This is because the parallel spin aligning energy is insufficient to overcome the thermal randomising effect, which rotates the spin direction. Such materials have a well-defined susceptibility, given by the Curie-Weiss Law

$$
\chi=\frac{c}{T-T_{C}}
$$


Here, $\mathrm{C}$ is the Curie constant described in Equation 2.6. The above Equation 2.10 is derived using "mean-field theory" (MFT) as presented in ref [132]. At low enough temperatures, all the moments in a ferromagnetic material are aligned in the same direction and it shows the highest magnetic response. In this case, materials become fully saturated and the highest value of magnetisation is called the saturation magnetisation. Saturation can also be reached at higher temperatures with large enough applied magnetic fields.

\subsubsection{Exchange interaction}

Ferromagnetic or antiferromagnetic materials exhibit magnetic ordering even in the absence of a magnetic field. Long-range magnetic ordering in these materials arises from the exchange interaction. Thus, the interaction between moments in ferromagnetic or antiferromagnetic materials are very important to understand.

The origin of the exchange interaction can be explained by considering a system of two electrons and calculating the total energy of the system. If the system has two electrons at position $r_{1}$ and $r_{2}$, then the Hamiltonian describing the energy of this system is

$$
H=H_{0}\left(\mathbf{r}_{1}\right)+H_{0}\left(\mathbf{r}_{2}\right)+\frac{e^{2}}{\left|\boldsymbol{r}_{1}-\boldsymbol{r}_{2}\right|}
$$

where $e$ is the electronic charge and $H_{0}$ is the one-electron Hamiltonian, and the third term is the electron-electron interaction.

It is important to mention that the spin states of the electrons need to be addressed in the wavefunctions. If the electron-electron interaction energy in Equation 2.11 is expected to be small compared with $H_{0}$, then this addition to the Hamiltonian can be treated with perturbation theory. It is also imperative to consider the constraint on the wavefunctions imposed by the Pauli exclusion principle. Since fermions such as electrons are indistinguishable, the wavefunctions must be antisymmetric if their positions and spin states are exchanged. The spins of electrons can have two possible combinations, antisymmetric antiparallel spins (singlet state, non-magnetic) or symmetric parallel spins (triplet state, magnetic). A symmetric spin state must be coupled with an antisymmetric spatial wave function and vice versa. The 
difference in energy between the two spatial states determines which spin state is favoured. This energy difference is referred to as the exchange energy $J_{i j}$ for two electrons at $i$ and $j$ sites.

The effect of the exchange interaction can be described by an effective Hamiltonian written directly in terms of the spins, known as the Heisenberg Hamiltonian, which can be represented as

$$
H_{H}=-\sum_{i j} J_{i j} S_{i} . S_{j}
$$

where $i$ and $j$ denote the sites of the two electrons involved and $S_{i}, S_{j}$ are the spin states of the two electrons at $i$ and $j$ sites. The term $J_{i j}$ defines the exchange energy between the two electrons at $i^{t h}$ and $j^{t h}$ sites as stated earlier. The sign of $J_{i j}$ determines whether ferromagnetic (parallel) or antiferromagnetic (antiparallel) arrangement of the spins is the lowest energy state for the system. The factor of 2 is omitted in Equation 2.12 because the summation includes each pair of spins twice. The other way of writing the above equation is

$$
H_{H}=-2 \sum_{i<j} J_{i j} S_{i} . S_{j}
$$

where the $i<j$ avoids the 'double-counting' of spins. Thus the factor of two returns again.

The above description can be explained by a simplified picture for a "direct exchange" interaction where the wavefunctions of the two nearest neighbour atoms or ions overlap with each other in order to cause the magnetic ordering without the need for an intermediary.

The direct exchange interaction is unlikely to be very effective for materials which exhibit insufficient direct overlap between neighbouring magnetic orbitals. Thus it is necessary to consider some kind of indirect exchange interaction to understand the magnetism of many magnetic materials. Especially, for rare-earth nitrides an indirect exchange interaction is important, because the $4 f$ electrons are strongly localised, so one would expect the direct $4 f$ $4 f$ inter-ion exchange interaction is negligible here. Instead, the exchange interaction proceeds indirectly through superexchange mechanism, where unoccupied $5 d$ states interact with the $4 f$ states through intra-ion $4 f-5 d$ exchange. It is believed that, ferromagnetic exchange exists 
between nearest neighbour (nn) rare-earth ions via the $4 f-5 d-5 d-4 f$ chain. A competing exchange channel is also considered for REN materials which is antiferromagnetic superexchange via the $\mathrm{N} 2 p$ states. In this case, the exchange mechanism follows the next nearest neighbour (nnn) chain, i.e. $4 f-5 d-\mathrm{N} 2 p-5 d-4 f$. Therefore, the overall exchange energy in RENs is comprised of two contributions, i.e. ferromagnetic and antiferromagnetic exchange but experimentally it is difficult to distinguish different contributions to $T_{c}$.

\subsubsection{Hysteresis loop and crystal anisotropy}

Ferromagnetic materials exhibit hysteresis in their magnetic response, as shown in Figure 2.2. A typical M-H diagram for a ferromagnet shows that as the applied field increases to a large enough value, the magnetisation of the material reaches the saturation point $\mathrm{C}$. Then as the applied field is reduced to a negative value the magnetisation begins to reverse, eventually producing saturation in the reverse direction at point $\mathrm{F}$, and finally the magnetisation increases back to zero once again $[127,128]$. A symmetrical closed loop known as a hysteresis loop is thus formed. A magnetic induction remains when the applied field reduces from saturation to zero and this is called the remanence magnetisation $M_{r}$. The reverse (negative) field needed to reduce the magnetic induction to zero is known as the coercivity or coercive field, $H_{c}$. Once the magnetisation reaches a maximum $\left(M_{S}\right.$, points $\mathrm{C}$ and $\left.\mathrm{F}\right)$ value, then increasing the field strength does not increase the magnetisation of the material any further. This phenomenon is known as saturation of the magnetic response, when all the moments are aligned in the direction of the field as described earlier [127, 128, 132].

Depending on the value of the coercivity, ferromagnetic materials are classified as either hard or soft. A hard magnet needs a large field to reduce its induction to zero (or conversely to saturate the magnetisation). A soft magnet is easily saturated, but also easily demagnetized.

The domain wall movement can be used to explain the nature of the hysteresis loop in ferromagnetic materials. The "domains", proposed by Weiss, are small regions in the ferromagnetic materials where electrons' magnetic moments are aligned in same direction. These are formed to minimise the free energy associated with magnetostatic energy, anisotropy energy and exchange energy [127, 131]. The magnetostatic energy originates from the interaction of the magnet with its own field or an external field. The anisotropy energy is the 
energy required to align electrons along a preferred axis known as the "easy-axis" in the crystal lattice. The exchange energy is associated with the spin alignment of electrons, i.e. either they are oriented in a same direction or in the opposite direction (see Equation 2.12). There is a competition between magnetostatic and exchange energy which tends to align the moments in antiparallel and parallel directions, respectively. A typical example of domain formation is shown in Figure 2.2(b).

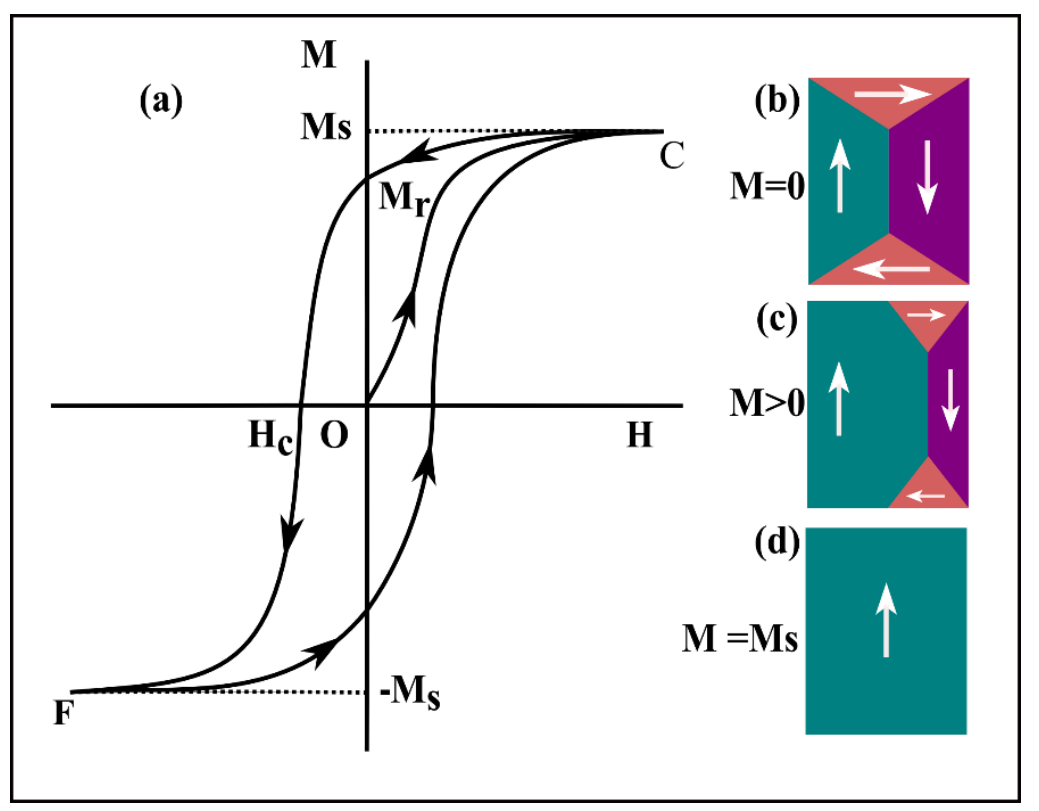

Figure 2.2: Hysteresis loop and domain structure formation for a ferromagnet below $T_{c}$ at varying magnetic field strength (Reproduced from [132]).

The net magnetisation is zero in the absence of a magnetic field as shown in Figure. 2.2(b). There is a finite magnetisation when a small magnetic field is applied as shown in Figure. 2.2(c). It results from the alignment of domains along the applied field direction leading to a non-zero magnetisation. As the magnetic field increases, domains flip towards the easy-axis (see the following section for more details) and at a high enough field all the domains are aligned in the applied field's direction, leading to a saturation condition $\left(M_{S}\right)$. Upon the reversal of magnetic field, the domains relax, resulting in a small magnetisation. There is a non-zero magnetisation even at zero magnetic field known as the remanent magnetisation $\left(M_{r}\right)$. A certain magnetic field, termed the coercivity or coercive field $\left(H_{c}\right)$, is required to completely randomise the domains leading to the net magnetisation once again being zero. Non-zero values 
of $M_{r}$ and $H_{c}$ are usually used as an indication of ferromagnetism in the magnetic materials. It is to be noted that, so far we do not have any clear experimental evidence to clearly identify the intrinsic easy and hard axes of REN materials.

\subsubsection{Shape anisotropy}

The magnetic anisotropy of a material is defined by the material's property to exhibit its saturation magnetisation in a preferred direction. This depends on the crystal structure and the shape of the material in the direction of magnetization. In ferromagnetism, each domain is spontaneously magnetized to saturation where the exchange energy plays a crucial role, but the direction of magnetisation varies from domain to domain. The overall direction of magnetisation could be on the easy axis or hard axis depending on the crystal structure, shape/size of the material and the direction of the applied magnetic field. Typically, for a small size film if the magnetic field is applied parallel to the film surface then most commonly the film will be easily magnetised whereas the perpendicular direction requires a much larger applied magnetic field to show the saturation magnetisation.

For example, we will consider two cases with the magnetic fields applied perpendicular and parallel to the GdN film surface. For the first case $\mathrm{GdN}$ requires a field of around $2.1 \mathrm{~T}(4 \pi M)$ to show the saturation magnetisation which is much higher than the coercive field estimated in this situation. But in the parallel case, the film will be easily saturated in a much lower applied magnetic field.

In this thesis, shape anisotropy is significant when correlating the magnetism and the Hall effect results in the ferromagnetic region. During Hall effect measurements magnetic fields are applied out-of-plane, i.e. perpendicular to the film surface, while the magnetic measurements presented in this thesis were obtained with the field in-plane. Therefore, one would be far away from getting the accurate results if one ignores the importance of shape anisotropy while tackling the Hall effect results. More details about the shape anisotropy correction and the Hall effect data fitting using the magnetic data has been mentioned in Chapter 6 (Low carrier concentration epitaxial GdN film). 


\subsection{Transport properties}

\subsubsection{Electrical transport in materials}

The electrical conductivity of materials arises due to motion of charged particles and it is influenced by the energy band structure. The nature of the energy bands categorises materials into several types and defines their conducting nature. The occupation of electron states in the energy bands follows the Fermi-Dirac distribution function where filling up of states starts from the lowest energy level to the next available energy states.

Crystalline solids are characterised by a perfect or nearly perfect periodicity of atomic structure. Each individual atom has different electronic shells. The closed electron shells, i.e. the core electrons of the individual atoms, are contained fully by the occupied bands with an even number of electrons whereas the higher lying states broaden into energy bands. The electrical properties depend on whether the highest occupied band is partially or completely filled with electrons. The large variation in band structures between different solids causes a wide range of electrical characteristics. Here we qualitatively begin to discuss some basic differences in electrical characteristics of materials caused by variations in band structure by considering some simplified energy bands. Most commonly, solids are classified into three types by their band structures: metal, semiconductor or insulator.

The number of energy states in a band is equal to the number of primitive cells $(N)$ in the crystal. As per Pauli's exclusion principle, there can be a maximum of two electrons to fill up each state. Therefore, $2 N$ valence electrons are needed to completely fill up a band. A monoatomic crystal with one valence electron in each atom can have two electrons per state if there are two atoms in the primitive cell. An atom with two valence electrons in a primitive cell can fill up the band completely. Solids, in which one or more bands are completely filled and all other bands are empty, act as insulators at $0 \mathrm{~K}$ [135].

However, if the number of electrons per primitive cell is an odd number, then the top-most occupied band will only be filled partially. This type of material allows current flow due to an applied electric field and is thus characterised as a good conductor of electricity, i.e. a metal. This is evident in alkali and noble metals which contain one valence electron in each primitive cell where the highest occupied state is exactly half-filled [135]. 
For insulators, the lowest-lying empty band (the conduction band) and uppermost filled band (the valence band) are separated by a significant energy gap with the Fermi level lying between the valence and conduction bands. In this case the valence band is completely full and the electrons available in the band cannot contribute to electrical conduction. On the other hand, the conduction band is empty and no electrons are available to contribute to electrical conduction. A significant amount of activation energy is required to activate the electrons in order to transit them from the valence band into the conduction band.

Semiconductors are a class of insulators having a similar band structure to insulators with the Fermi level lying inside the band gap. But the band gap of semiconductors is relatively narrow compared to that of insulators and lower activation energy is required for the transition of electrons from the valence band into the conduction band. In the ground state at $0 \mathrm{~K}$, the conduction band does not contain any free electrons and the semiconductor acts as an insulator. However, an increase in temperature thermally activates the higher states of the valence electrons to overcome the band gap and enter the conduction band. This implies that conductivity increases with an increase of temperature. In this process the valence electrons leave empty states in the valence band called holes when the transition to the conduction band occurs.

Therefore, two phenomena occur upon the application of an electric field: first, the electrons that are already present in the conduction band acquire additional energy to move to unoccupied levels within the same band and second, the vacancies (holes) created by the electrons (that moved to the conduction band) represent unoccupied levels in the valence band. In this case, the electrons below the holes gain energy to jump up and occupy them. Thus holes move to a lower energy state in the same band (valence band) and contribute to the electric current. Therefore, for semiconductor materials there is a limited flow of current across the crystal and the electrical conductivity lies between metals and insulators. 


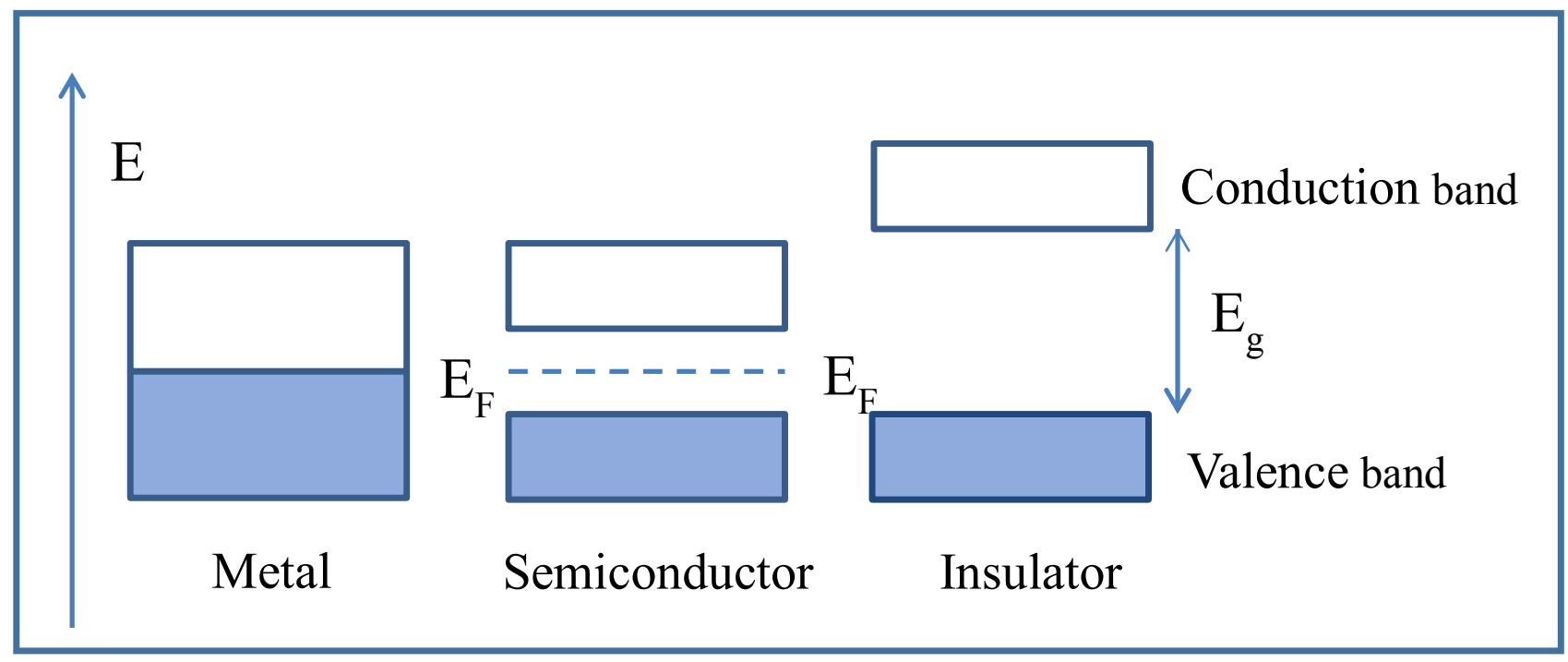

Figure 2.3: Simplified illustration of the electronic energy levels for a metal, semiconductor and insulator. $\mathrm{E}_{\mathrm{F}}$ and $\mathrm{E}_{\mathrm{g}}$ represent the Fermi level and band gap, respectively.

Further discussion of electrical properties of materials are elaborated from the classical Drude model of metallic conduction. The following section will describe the classical Drude model and from this model we will see how the conductivity of a material depends on different parameters.

\subsubsection{Classical Drude model of metallic conduction}

The conductivity of a metallic system was explained by Drude in 1900 by considering the ideas of the kinetic theory of gases [132]. According to his model the metal is considered to consist of a gas of electrons and those electrons tend to move randomly in the absence of an external electric field. As a result, the net motion of electrons is zero; hence no current flows in the metal. When an electric field $\vec{E}$ is applied to a metal, the electrons move in a specific direction under the influence of the electric field. Therefore the distribution function of the electrons in the metal undergoes a change. The Drude model of electrical conductivity assumes that the charge carriers in a metal are free particles that occasionally experience scattering events while propagating throughout the media [132]. Hence the scattering limits the electrons' overall 
motion under an applied electric field [132]. According to the Drude model, the conductivity is given by:

$$
\sigma=\frac{e^{2}}{m} n \tau
$$

and the electrical resistivity $\rho$ is the reciprocal of conductivity and expressed by:

$$
\rho=\frac{1}{\sigma}=\frac{m}{n e^{2} \tau}
$$

Here $n$ is the number of free electrons per unit volume, $m$ is the mass of the electron, and $\tau$ is the average relaxation time between two successive scattering events.

Sommerfeld extended the Drude model in terms of quantum mechanical effects and replaced the electron mass $m$ with an effective mass $\left(m^{*}\right)$ for electrons in the solid [132]. From Equation 2.14 it is clear that the conductivity mainly depends on the number of charge carriers and the relaxation time; both are functions of temperature and magnetic field. In the results chapters we will discuss briefly how the temperature and magnetic field affect the conductivity.

Ideally, if we consider a pure material with no defects, then at absolute zero temperature the electrons can travel through the crystal lattice without any collisions. The mean free path $l=$ $v_{f} \times \tau$ would then be infinite ( $v_{f}$ is the Fermi velocity) and the resistivity becomes zero. In reality, the relaxation time is never infinite.

The classical Drude model considers the relaxation time to be independent of temperature and the mean free path to be of the order of the interatomic distance [128]. The resistance was assumed to be proportional to the square root of the absolute temperature [128]. However, experimental results show that the specific resistance varies more nearly linearly with $T$ in metals due to the electron scattering from phonons (lattice vibrations).

According to Mathiessen's rule $[132,134]$ the total scattering rate of a material is defined as:

$$
\frac{1}{\tau}=\frac{1}{\tau_{i}}+\frac{1}{\tau_{p}}
$$

Here $\frac{1}{\tau_{i}}$ is the scattering rate due to the scattering of electrons from impurities or defects, and $\frac{1}{\tau_{p}}$ is the rate due to electron-phonon scattering. At high temperature there are more phonons 
than at low temperature, so $\frac{1}{\tau_{p}}$ increases with temperature. As a result the resistivity increases with increasing temperature. The electrons still scatter from lattice disorder or impurities even at very low temperatures, so a finite residual resistivity is observed as temperature goes to zero.

A special type of metal is a semimetal. The bands of a semimetal tend to be very similar to semiconductor bands but with a very small indirect overlap between the bottom of the conduction band and the top of the valence band, i.e. a little portion of the conduction band overlaps below the Fermi level and the valence band sticks up just above the Fermi level. Therefore, in the ground state a small number of conduction-band states are occupied and an equal number of valence-band states are unoccupied.

Semiconductors are classified in two categories, namely intrinsic semiconductors or extrinsic semiconductors. First we will discuss pure or intrinsic semiconductors followed by extrinsic semiconductors.

As mentioned earlier, there are two types of charge carriers available in a semiconductor, electrons (with density $n_{e}$ ) in the conduction band and holes $\left(n_{h}\right)$ in the valence band, and they are equal but opposite in sign. The intrinsic carrier concentration of electrons $\left(n_{i}\right)$ and holes $\left(n_{i}\right)$ depends on temperature and is determined by the thermal excitation of electrons from the valence band to the conduction band,

$$
n_{i}=N_{C} \exp \left[\frac{-E_{g}}{2 k_{B} T}\right]
$$

Here $N_{c}$ is a parameter dependent on the semiconducting nature of the material, $E_{g}$ is the band gap, and $k_{B}$ is the Boltzmann constant. The factor of 2 occurs because the Fermi level lies in the middle of the band gap for intrinsic semiconductors.

The conductivity of an intrinsic semiconductor depends on both the carrier concentration and the scattering rate $(\tau)$. The carrier concentration increases exponentially with temperature and this typically dominates the temperature dependence of the scattering rate. Increasing the temperature decreases the resistivity of an intrinsic semiconductor.

When impurity atoms or defects are incorporated into a pure semiconductor crystal, its electrical properties are altered. In this case, additional electronic levels are created from the 
impurity atoms in the band gap. This type of material is called on extrinsic semiconductor or doped semiconductor. For doped semiconductors, the materials' impurity levels play an important role in determining the type of charge carriers. For $n$-type doping impurity levels donate extra electrons to the conduction band, whereas for $p$-type doping impurity levels accept electrons from the valence band, leaving extra holes in the valence band.

Depending on the type of doping, the impurity levels can be created near the conduction band or near the valence band. At low doping concentrations, the isolated localised impurity levels form below the conduction band (for donors) and charge carriers (electrons) transit to the conduction band through thermal excitation. In this case, the position of the Fermi level is determined by the concentration of the impurity atoms and the fraction of the dopant electrons that are excited to the conduction band.

The carrier concentration $(n)$ is then determined by:

$$
n=N_{d} \exp \left[\frac{-\left(E_{C}-E_{F}\right)}{k_{B} T}\right]
$$

Here $N_{d}$ is a parameter that depends on the band structure and temperature of the material, $E_{C}$ is the conduction band minimum energy, and the Fermi level $E_{F}$ is determined by the impurity energy level $E_{d}$.

Similar to intrinsic semiconductors, the conductivity of a doped semiconductor also depends on both the charge carrier concentration and the relaxation time. For doped semiconductors with donors, as temperature increases, more electrons from impurity levels are excited to the conduction band through thermal activation. Therefore, as the temperature increases, the carrier density in the conduction band increases.

As the doping concentration in $n$ - type material increases significantly, the impurity levels form a band near the bottom of the conduction band and it can even merge with the conduction band edge,. In this case, the Fermi level lies very close to the conduction band edge or even inside the conduction band (forming a degenerately doped semiconductor), therefore no thermal activation is required to activate the charge carriers. The degenerately doped semiconductor behaves like a metal with low carrier concentration. 


\subsubsection{Hall effect}

Conductivity measurements alone are not sufficient to uniquely determine both the number or even the sign of the conducting charge carriers $n$ and their mobility $\mu$. The Hall effect measurement is important in this regard, to determine the number of charge carriers in the sample and their sign. When a magnetic field is applied perpendicular to a conductor carrying current, a voltage is developed across the specimen in a direction perpendicular to both the current and the magnetic field. This phenomenon is known as the Hall effect [128, 132 - 135].

\subsubsection{Hall voltage and Hall coefficient}

Consider a slab of material subjected to an external electric field $\vec{E}_{x}$ along the x- direction and a magnetic field $\vec{B}_{z}$ along the z- direction as shown in Figure 2.3. Due to the electric field a current density $\vec{j}_{x}$ will flow in the direction of $\vec{E}_{x}$. For simplicity, first we will consider the case in which the current is carried by electrons. Later we will explain it for both charge carriers, that is for electrons and for holes.

The electric force on the electron having a charge $-e$, is $-e \vec{E}$. Under the influence of a magnetic field, the conduction electrons experience a Lorentz force

$$
\vec{F}_{B}=-e \vec{v} \times \vec{B}
$$

Thus, the total force acting on the electron is

$$
\vec{F}_{B}=-e \vec{E}-e \vec{v} \times \vec{B}
$$

where, $\vec{v}$ is the drift velocity of the electrons and $\vec{B}$ is the applied magnetic field. The Lorentz force tends to deflect the electrons downwards and this results in the build-up of a negative charge density on the lower surface and a positive charge on the upper surface of the conductor. The accumulation of charge on the surface of the conductor continues until the force on charge carriers due to the electric field associated with it is large enough to cancel the force exerted by the magnetic field. Finally, a steady state is obtained when the current along the y-axis vanishes and a field $\vec{E}_{y}$ is set-up. 


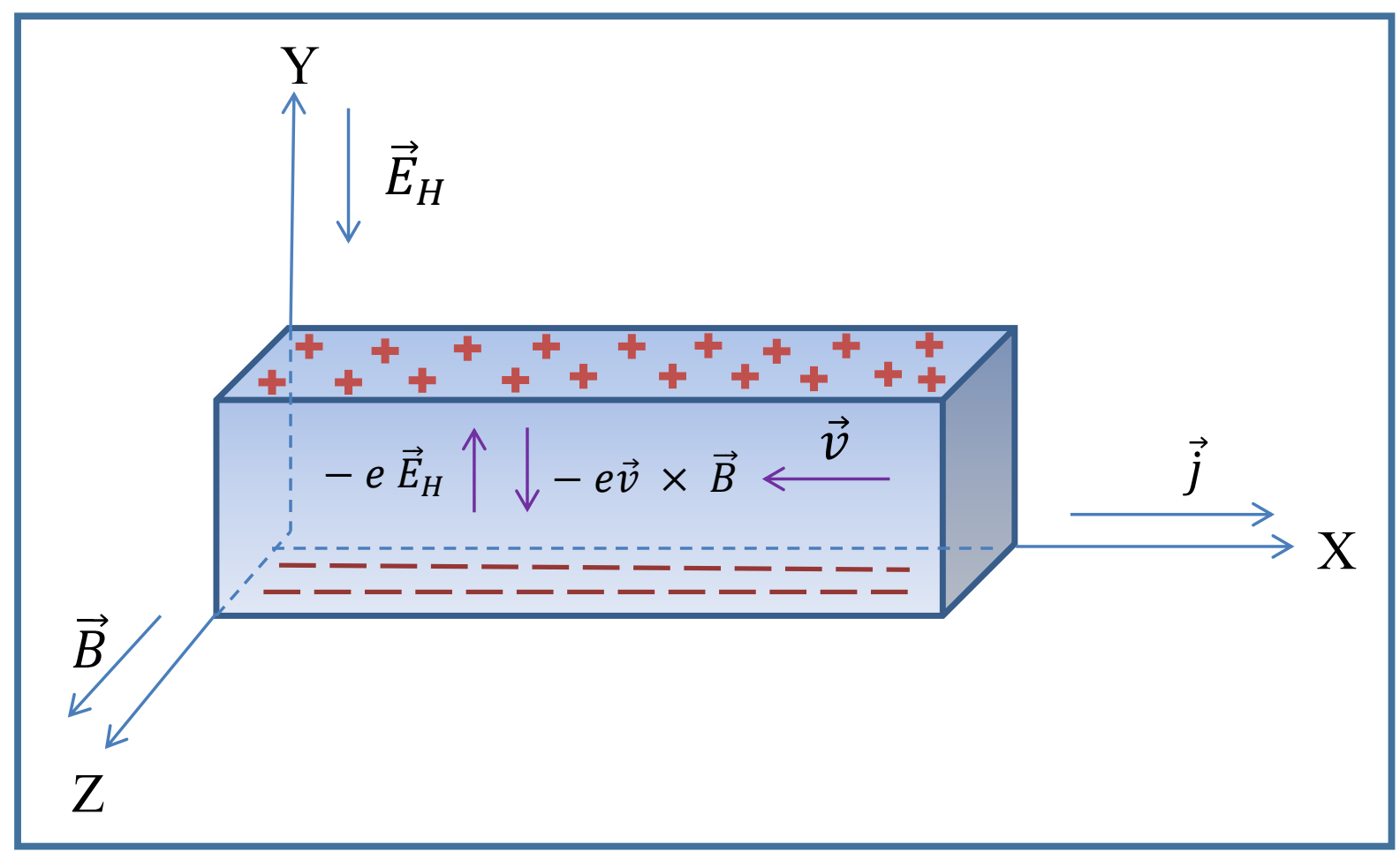

Figure 2.4: Geometry of the Hall effect. The Lorentz force $-e \vec{v} \times \vec{B}$ on the electrons is balanced by the force $-e \vec{E}_{H}$ due to the Hall field $\vec{E}_{H}$.

In our case,

$$
\vec{F}_{y}=-e \vec{E}_{y}+e \vec{v}_{x} \vec{B}_{z}
$$

In steady state, $\vec{F}_{y}=0$, hence Equation 2.21 can be written as,

$$
0=-e \vec{E}_{y}+e \vec{v}_{x} \vec{B}_{z}
$$

From the above equation, we can write,

$$
\vec{E}_{y}=\vec{v}_{x} \vec{B}_{z}
$$

This $\vec{E}_{y}$ gives rise to the Hall voltage $\left(\vec{V}_{H}\right)$ or the Hall field.

The current density is given by:

$$
\begin{aligned}
& \vec{j}_{x}=-n e \vec{v}_{x} \\
& \vec{v}_{x}=-\frac{\vec{j}_{x}}{n e} \ldots
\end{aligned}
$$

Substituting the value of $\vec{v}_{x}$ in Equation 2.23 we get

$$
\vec{E}_{y}=-\frac{\vec{j}_{x} \vec{B}_{z}}{n e}
$$




$$
\begin{aligned}
& R_{H}=\frac{\vec{E}_{y}}{\vec{j}_{x} \vec{B}_{z}}=\frac{1}{-n e} \\
& R_{H}=-\frac{1}{n e} \ldots \ldots \ldots
\end{aligned}
$$

Equation 2.28 represents the Hall coefficient, whose measurement can be used to obtain $n$, the charge carrier density. The above expression 2.28 is derived for the ordinary Hall effect that applies to non-magnetic materials, containing only one type of charge carrier. The carrier concentration may be temperature and magnetic field dependent, so

$$
R_{H}(T, \vec{B})=-\frac{1}{n(T, \vec{B}) e}
$$

\subsubsection{Anomalous Hall effect (AHE)}

Another significant contribution to the Hall effect is the anomalous Hall effect (AHE) or extraordinary Hall effect (EHE) which is usually observed in magnetic materials and it depends directly on the magnetisation of the material. Hence, the Hall resistance of magnetic materials appears due to two contributions, the ordinary Hall effect and the anomalous Hall effect, as given by:

$$
\rho_{x y}=R_{H} \vec{B}+R_{A} M(T, \vec{B})
$$

where $R_{H}$ represents the ordinary Hall effect which is proportional to the magnetic field $B$ and inversely proportional to the number of charge carriers in the sample. The term $R_{A}$ stands for the anomalous Hall effect, which is proportional to the magnetisation of the sample and has a significant effect below the $T_{c}$.

The physics behind the origin of anomalous Hall effect is quite complicated, being related to the spin-orbit interaction. Initially, the literature suggested that the AHE was either an extrinsic effect due to spin-dependent scattering of the charge carriers or an intrinsic effect related to their Berry phase curvature originating from the time-dependent electron motion in ferromagnets [136, 137].

The AHE has been most routinely investigated in magnetic metals and in conventional semiconductors doped heavily with magnetic ions [137]. Significant research has not yet been done in defect-free materials with controlled electron concentrations to reveal fully the 
influence of the spin-orbit interaction on the transport of charge and spin. In this regard, the RENs are promising candidates for this study because they offer a systematically varying spinorbit interaction strength across the 14-member series. Recent attempts to understand the anomalous Hall effect on rare-earth nitrides within the experimental context and a semiclassical theoretical treatment has potentially identified the highly spin polarised nature of the conducting channel [138]. Below we explain the core concept behind it.

The magnitude of the anomalous Hall conductivity $\sigma_{x y}^{E H}$ is proportional to the spin difference between the concentration of spin-up $\left(n_{\uparrow}\right)$ and spin-down $\left(n_{\downarrow}\right)$ conduction electrons, i.e. $\left(n_{\uparrow}-n_{\downarrow}\right)$. Once a sample has been fully magnetised its spin difference is also maximised, and as a result one would expect to see the contribution of the anomalous Hall effect is also maximised. In contrast, the longitudinal conductivity is proportional to the total carrier concentration, i.e. $\left(n_{\uparrow}+n_{\downarrow}\right)$. The Hall resistivity is $\rho_{x y}=\frac{\sigma_{x y}}{\sigma_{x x}^{2}} \propto \frac{\left(n_{\uparrow}-n_{\downarrow}\right)}{\left(n_{\uparrow}+n_{\downarrow}\right)^{2}}$, [138] where it is assumed that the perpendicular conductivity $\left(\sigma_{x y}\right)$ is much smaller than the longitudinal conductivity $\left(\sigma_{x x}\right)$, i.e. $\left(\sigma_{x x}\right) \gg\left(\sigma_{x y}\right)$. If we have electrons with only a single type of spin, i.e. majority-spin or spin-up $\left(n_{\uparrow}\right)$ electrons, then the contribution from the minority-spin or down spin is zero $\left(n_{\downarrow}=0\right)$. This will give us $\rho_{x y} \propto \frac{\left(n_{\uparrow}\right)}{\left(n_{\uparrow}\right)^{2}} \propto \frac{1}{n}$, which is inversely proportional to the carrier concentration similar to the ordinary Hall effect. Understanding this concept can potentially tell us which spin channel (majority or minority) dominates the conductivity of the film. Especially, this is important for moderately doped RENs where conductivity is dominated by a single spin channel before the minority-spin band begins to fill which. We will see this in our RENs samples presented in the result sections. 


\subsubsection{Magnetoresistance}

Apart from the Hall effect, the applied magnetic field also has a significant effect on the resistivity of a sample which is known as magnetoresistance. The magnetoresistance (MR) of a material is defined as the ratio of change in the resistance under an applied magnetic field to the resistance in zero field $[128,132]$. It is expressed as

$$
\mathrm{MR}=\frac{\Delta R}{R}=\frac{\rho(H, T)-\rho(0, T)}{\rho(0, T)}
$$

where $\rho(H, T)$ and $\rho(0, T)$ represent the electrical resistivities at temperature $T$ with and without the applied magnetic field $H$, respectively. Magnetoresistance can be longitudinal or transverse according to the direction of the applied magnetic field. When the magnetic field is applied normal (perpendicular) to the current flow, the effect is termed as transverse magnetoresistance whereas longitudinal magnetoresistance occurs when the applied magnetic field is applied parallel to the current [134]. There are many sources that could influence the magnetoresistance of a material, such as changes in carrier concentration, relaxation time, or changes in the distribution of energy states $[139,140]$.

Magnetoresistance can be positive or negative. Positive magnetoresistance often occurs in nonmagnetic metals originating from the Lorentz force, where electrons are forced to move on spiral trajectories. This process subsequently reduces the longitudinal path of the electrons between two successive collisions. Therefore an increase in resistance is observed [133]. In this scenario the magnitude of the magnetoresistance is typically proportional to $H^{2}$ in which $H$ is the applied magnetic field perpendicular to the current direction.

Several explanations have been proposed to describe the occurrence of negative magnetoresistance. In magnetic materials it can originate from spin-dependent scattering of conduction electrons from localised magnetic moments such as in dilute metallic alloys [141, 142]. This phenomenon also occurs for ferromagnets [7, 139, 143] and in semiconducting films [144]. The non-degenerate Anderson model and the Kondo model have been considered to describe this spin-dependent scattering interaction of conduction electrons. In this case the spin flip scattering of the localised magnetic ions is dominated by the applied magnetic field and this domination causes the negative magnetoresistance [145]. 
A few decades ago (1968), B. T. Beal-Monod and R. A. Weiner studied the negative magnetoresistance in dilute alloys [146]. Their study found that the effect of magnetic impurities in dilute alloys was the main the cause of negative magnetoresistance. In the same study, authors calculated the MR value using the second Born approximation using an $s$ - $d$ exchange model where the negative MR at low field is proportional to the square of the magnetization [146]. Later on R. P. Khosla et al. proposed a semi-empirical expression of the form

$$
\frac{\Delta \rho}{\rho}=-B_{1} \ln \left(1+B_{2}^{2} H^{2}\right)
$$

to describe the negative magnetoresistance in the low field regime in degenerate CdS samples [144]. The terms, $B_{1}, B_{2}$ in Equation 2.32 represents fitting parameters and $H$ stands for applied magnetic field, respectively.

There are other several possibilities origins of negative MR. Majumdar et al. observed a negative MR in ferromagnetic metals [140]. According to their interpretation, the suppression of spin disorder scattering under an applied magnetic field causes a negative MR and a maximum negative MR value was shown near the transition temperature $\left(T_{c}\right)$ [140]. A strong negative MR near $T_{c}$ can also be seen in ferromagnetic semiconductors where an applied magnetic field enhances the exchange splitting and reduces the band gap; causing a significant reduction of resistance. For example, the resistance of EuO changes by many orders of magnitude in the presence of a magnetic field [147].

Spin-dependent tunnelling between magnetic domains separated by insulating grain boundaries has also been used to explain the cause of negative magnetoresistance in several granular ferromagnetic systems [148, 149]. An applied field aligns the domains and enhances the tunnelling probability and thereby the resistance of the sample reduces. This effect is most prominent near the transition temperature $\left(T_{c}\right)$ where one can see the strongest magnetic disorder. 


\subsection{Thermopower}

\subsubsection{Definition and the physical origin of thermopower}

The thermopower or thermoelectric effect is the direct conversion of a temperature difference to electric voltage and vice-versa. It is often said to measure the "entropy per carrier" [150].

If a continuous circuit is formed between two dissimilar electrical conductors, as shown below in Figure. 2.5, a temperature difference between the two junctions produces a voltage difference between the two substances and an electric current will flow continuously in the closed circuit. This is the famous Seebeck effect, and its magnitude is determined by the scattering rate and the density of the conduction electrons [151, 152].

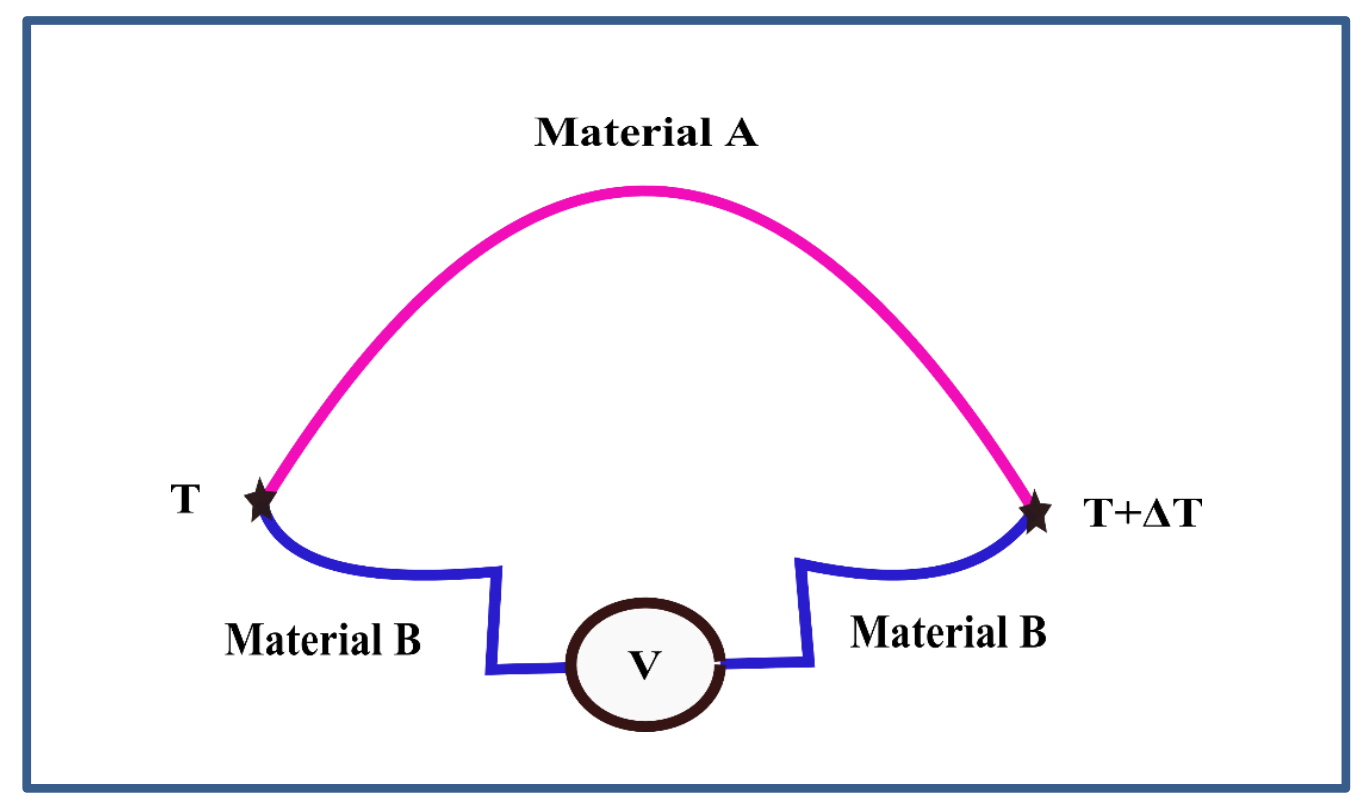

Figure 2.5: A thermoelectric circuit.

For an isolated conductor, when a temperature gradient $(\vec{\nabla} T)$ develops along the conductor, then an electric field $(\vec{E})$ will be generated along the conductor according to the Seebeck law. This electric field $\vec{E}$ is proportional to the $\vec{\nabla} T$ across the conductor. The Seebeck coefficient, $S$, is then defined as the ratio of the electric field $\vec{E}$, to the temperature gradient $\vec{\nabla} T$.

$$
\vec{E}=(S \vec{\nabla} T)_{\vec{J}=0}
$$


The proportionality constant $S$ is called the thermopower or the Seebeck coefficient of the material which is mostly written in the following format

$$
S=\frac{\Delta V}{\Delta T}
$$

where $\Delta V$ is the voltage difference and $\Delta T$ is the temperature difference across the sample.

The voltages produced by the Seebeck effect are typically small, usually only a few microvolts per Kelvin of temperature difference.

The physical origin of the thermopower can be described in the following manner. At finite temperatures, we can consider the electrons as particles that can move continually in a random direction. If the two ends of the material are in a thermal equilibrium $(\Delta T=0)$, then the probability of an electron moving from one end to the other is the same as for an electron travelling the opposite direction. Thus, there will be no net flux of electrons.

If an electrically isolated conductor $(\vec{J}=0)$ is placed under the influence of a temperature gradient $\vec{\nabla} T$, the electrons at the hot end have a higher average energy than those at the cold end (Figure 2.6). Hence, the average speed of the electrons is greater at the hot end than the cold end. As a consequence there is a net diffusion of electrons down the temperature gradient, which piles up negative charge and creates a surplus of electrons at the cold end and positive charge at the hot end, leading to a thermopower sign that corresponds to the carrier sign. This charge distribution generates an internal electric field $\vec{E}$.

This process will continue until the induced electric potential between the two ends is large enough to halt the net flow of electrons. The steady state is achieved, once the temperature gradient and the electrostatic repulsion are equal, as a result the net current flow will be stopped.

The proportionality factor between the electric field $\vec{E}$ and the temperature gradient $\vec{\nabla} T$ in the steady state is called the diffusion thermopower, $S_{d}$, [153] which is discussed in more detail in the following section. 


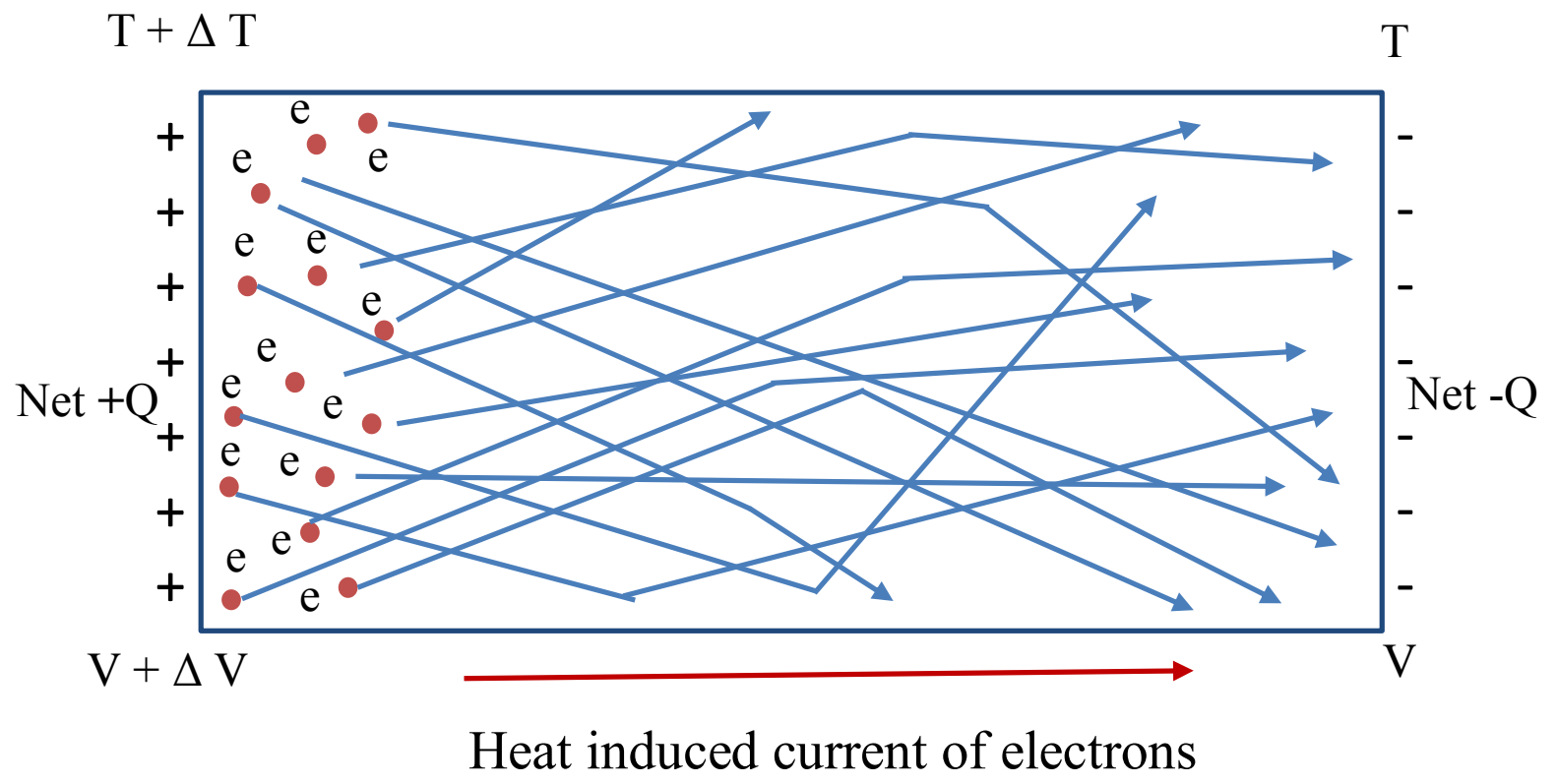

Figure 2.6: Simplified illustration of the diffusion of electrons caused by a temperature gradient. An electrically isolated slab of material is heated on the left $(\Delta T)$ causing a net flux of electrons towards the right.

\subsubsection{Diffusion thermopower}

The diffusion thermopower $\left(S_{d}\right)$, is most commonly used in the Mott formula which was derived by Mott (Mott and Jones 1936) from the Boltzmann equation in the relaxation time approximation [153]. It is expressed as:

$$
S_{d}=\frac{\Pi^{2} k_{B}^{2} T}{3 e}\left(\frac{\partial \ln \sigma\left(\varepsilon_{f}\right)}{\partial \varepsilon_{f}}\right)
$$

where $k_{B}$ is the Boltzmann constant, $-e$ is the electronic charge, $T$ is the absolute temperature and $\sigma\left(\boldsymbol{\varepsilon}_{f}\right)$ is the electrical conductivity as a function of hypothetical Fermi level, $\boldsymbol{\varepsilon}_{f}$. 
The conductivity in turn depends on the Fermi energy via the carrier concentration and the relaxation time. Using the simplest argument, one might expect these parameters to vary on an energy scale given by the energy separation between the band extrema and the Fermi energy, i.e. the Fermi energy measured, as is anyway conventional, from the closest band extremum.

The derivative of $\frac{\partial \ln \sigma\left(\varepsilon_{f}\right)}{\partial \varepsilon_{f}} \sim \frac{\alpha}{\varepsilon_{f}}$, so the thermopower is then of order

$$
S_{d} \sim \frac{\Pi^{2} k_{B}^{2} T}{e}\left(\frac{\alpha}{\varepsilon_{f}}\right)
$$

The above Equation 2.36 could guide us to obtain the diffusion thermopower $S_{d}$ from where one can roughly estimate the Fermi energy. The value of $\alpha$ is close to unity at high temperatures and crudely assumed to be $\frac{1}{3}$ at low temperatures $[153,154]$. Thus, if the thermopower is proportional to the absolute temperature it is probable that this degenerate-gas interpretation is appropriate.

One can further build up an argument concerning the value of $\alpha$. The bits of conductivity that depend on $\boldsymbol{\varepsilon}_{f}$ are the electron density and relaxation time, i.e. $n$ and $\tau$, respectively. In a real band with real scattering the conductivity strongly depends on the product of $n \tau$ which is eventually proportional to the value of $\varepsilon_{f}^{\alpha}$ for $\alpha$ close to 1 [154].

The above discussion makes clear that the thermoelectric measurement is useful to study the sign of the charge carriers, roughly estimate the Fermi energy and the relaxation time. Especially, the thermopower measurement process is in contrast to the other transport measurement, e.g., resistivity (as a function of temperature), where it simply measures the sum of the total scattering (electron - electron, electron - phonon and phonon - phonon) and cannot provide us any clue about the Fermi energy. Therefore, the thermopower experiment provides a unique opportunity to understand the conducting state of a material. 


\section{Chapter 3}

\section{Sample preparation and characterisation}

This chapter describes sample preparation and characterisation techniques of rare-earth nitride (REN) samples grown for this thesis. Both polycrystalline and epitaxial films were used to carry out the magnetic and transport measurements. The epitaxial GdN and $\mathrm{SmN}$ were obtained from our collaborators in Valbonne, France, whereas polycrystalline GdN and SmN films were grown at the Victoria University of Wellington (VUW). Note that our collaborators in France have been successfully growing epitaxial REN films over the last few years in their wellequipped and most advanced MBE lab. Their state-of-the-art research facilities and cuttingedge research techniques for REN films growth help them to successfully produce a series of good quality epitaxial films. Some of their research results are already highlighted in the peer review article $[108,110]$.

This chapter has two main parts, sample preparation and characterisation. The chapter has been structured as follows: In the first part, we describe the general overview of sample preparation. Under this part we have discussed the important growth parameters and how they have been considered for RENs growth. Section 3.2 and 3.3 sketches the structure of the ultra-high vacuum (UHV) growth chamber and growth procedure, respectively.

Section 3.4 presents the structural and compositional characterisation of the films. This section has categorised in two subsections i.e. 3.4.1 and 3.4.2, respectively. The first subsection contains the study of X-ray diffraction (XRD) whereas the emphasis has been given to Rutherford backscattering spectrometry (RBS) in the last subsection. X-ray diffraction was used extensively to determine the crystalline nature of the RENs, whereas Rutherford backscattering spectrometry was used to determine the film composition and thickness estimation. 


\subsection{General overview of sample preparation}

The growth of rare-earth nitride thin films is a particularly challenging task. Preparing good quality thin films of rare-earth nitrides is required to obtain reliable experimental data. Growing REN films demands a very clean atmosphere because oxygen impurities could have detrimental effects on the properties of the RENs. There are several challenges to achieve a good quality film, such as preparing the samples with the correct stoichiometry between the rare-earth metal and nitrogen, low defect density and low impurity concentration [15, 24]. These difficulties can be minimized by following a careful REN preparation technique.

Different techniques have been followed for preparing the RENs, but pulsed laser deposition, sputtering and physical vapour deposition (PVD) methods are the most common. One wellknown preparation technique (PVD) is the evaporation of rare-earth elements at a high temperature, with a partial pressure of approximately $1 \times 10^{-4}$ mbar pure nitrogen gas [15, 44].

The growth of REN thin films depends on a range of free parameters. The choice of the substrate on which the film will be deposited, the growth temperature, the nitrogen partial pressure and the capping layer are all very important for producing (growing) a high-quality REN film for transport, magnetic or optical measurements. The following section describes how different parameters can significantly influence the quality and properties of REN samples.

\subsubsection{Substrate}

The choice of a suitable substrate is very important for REN growth, especially it is quite challenging for epitaxial growth due to lattice mismatch of the RENs with the substrate. The crystallographic orientation of a substrate influences the growth orientation of the film and the substrates lattice constant affects the strain on the first layers of growth. Most commonly we use sapphire $\left(\mathrm{Al}_{2} \mathrm{O}_{3}\right)$, silicon, $\mathrm{GaN}, \mathrm{MgO}$ and yttria stabilized zirconia (YSZ), as they are commercially available in large quantities $[15,24,69,92,105]$. In this thesis, we have used a range of substrates according to our research requirements, such as $\mathrm{Si}, \mathrm{GaN}, \mathrm{YSZ}, \mathrm{Al}_{2} \mathrm{O}_{3}$ and glass. At the beginning of each result chapter, we have mentioned which substrate was used for the particular $\mathrm{GdN}$ and $\mathrm{SmN}$ films growth. 
Si has relatively low lattice mismatch (around 6\% for the case of GdN and $\mathrm{SmN}$ ) but this substrate has a tendency to form a rare-earth silicide at high temperatures at the interface between the rare-earth nitrides and the silicon, which is a major drawback of using it for preparing REN films. To overcome this difficulty AlN buffer layers were grown on Si substrate before depositing REN layers. This technique has successfully tested and reported in the literature $[88,107,108,110]$.

The other promising material yittria stabilized zicronia (YSZ) has a face centre cubic structure and shows low lattice mismatch for $\operatorname{GdN}(3 \%)$ and $\operatorname{SmN}(2 \%)$ but it can develop a thin oxide layer at the interface at higher temperatures. It was found that at a temperature close to $600^{\circ} \mathrm{C}$, oxygen mobilise in YSZ resulting in the formation of an oxide layer at the interface [109]. But REN grown below this temperature can provide reliable results which we have shown in the results section (Chapter 8). Note that this substrate exhibits lower thermal conductivity [155] which makes it an ideal candidate for the thermopower measurement of thin films. The brief discussion about it has been mentioned later in the thermopower Section 4.3.7.

$\mathrm{Mg}_{\mathrm{g}} \mathrm{O}$ substrate which also exhibits cubic structure similar to the RENs has been used for epitaxial growth. But the lattice mismatch with the RENs is very high, about $20 \%$ in the case of GdN [156].

\subsubsection{Capping layer}

The RENs react easily with the ambient atmosphere $[15,21]$. So before attempting any ex-situ measurement we need to protect the film by an extra layer, which is called the capping layer, the passivating layer or the protecting layer. An informed choice of a capping layer (like substrate) is important to constrain the range of experiments that will be possible with that film. Mostly AlN or GaN were reported as a capping layer due to various reasons [24, 53, 107, 110]. Especially, their very small conductivities make them more reliable for conducting transport measurements $[15,24,53,96]$. As this thesis is mainly focused on transport properties, all our samples were capped by GaN. GaN shows good chemical stability over time which ensures us to keep samples safe from oxidisation for a longer period to conduct many measurements. 


\subsubsection{Growth temperature and pressure}

The temperatures and pressure are two important parameters for RENs growth. Epitaxial growth must be performed at temperatures of several hundred degrees in contrast to polycrystalline growths which occur at room temperature. At higher temperatures $\left(400^{\circ} \mathrm{C} \sim\right.$ $750^{\circ} \mathrm{C}$ ) the mobility of atoms is enhanced significantly leading to greater re-arrangements of the atoms on the substrates.

The growth pressure can change the films' properties significantly. During the growth, the nitrogen pressure inside the growth chamber was controlled by the instrument operator in such a way to produce films known to have a significant concentration of nitrogen vacancies. Otherwise, we would have produced a REN film heavily doped with nitrogen vacancies. The main aim of this thesis is to investigate the transport properties of RENs where nitrogen vacancies play a crucial role in controlling the carrier concentration as described briefly in the result section (Chapter 5, Section 5.1). The specific growth conditions used for films studied here were empirically chosen based on previous film growths [107 - 110] in order to get carrier concentrations within a desired range $\left(10^{19} / \mathrm{cm}^{3} \sim 10^{21} / \mathrm{cm}^{3}\right)$.

\subsection{Structure of the UHV growth chamber}

Two polycrystalline REN films were grown at VUW using a Thermionics ultra-high vacuum (UHV) system. Figure 3.1 shows the schematic diagram of the ultra-high vacuum deposition chamber used for rare-earth nitride growth in the MBE lab at VUW. 


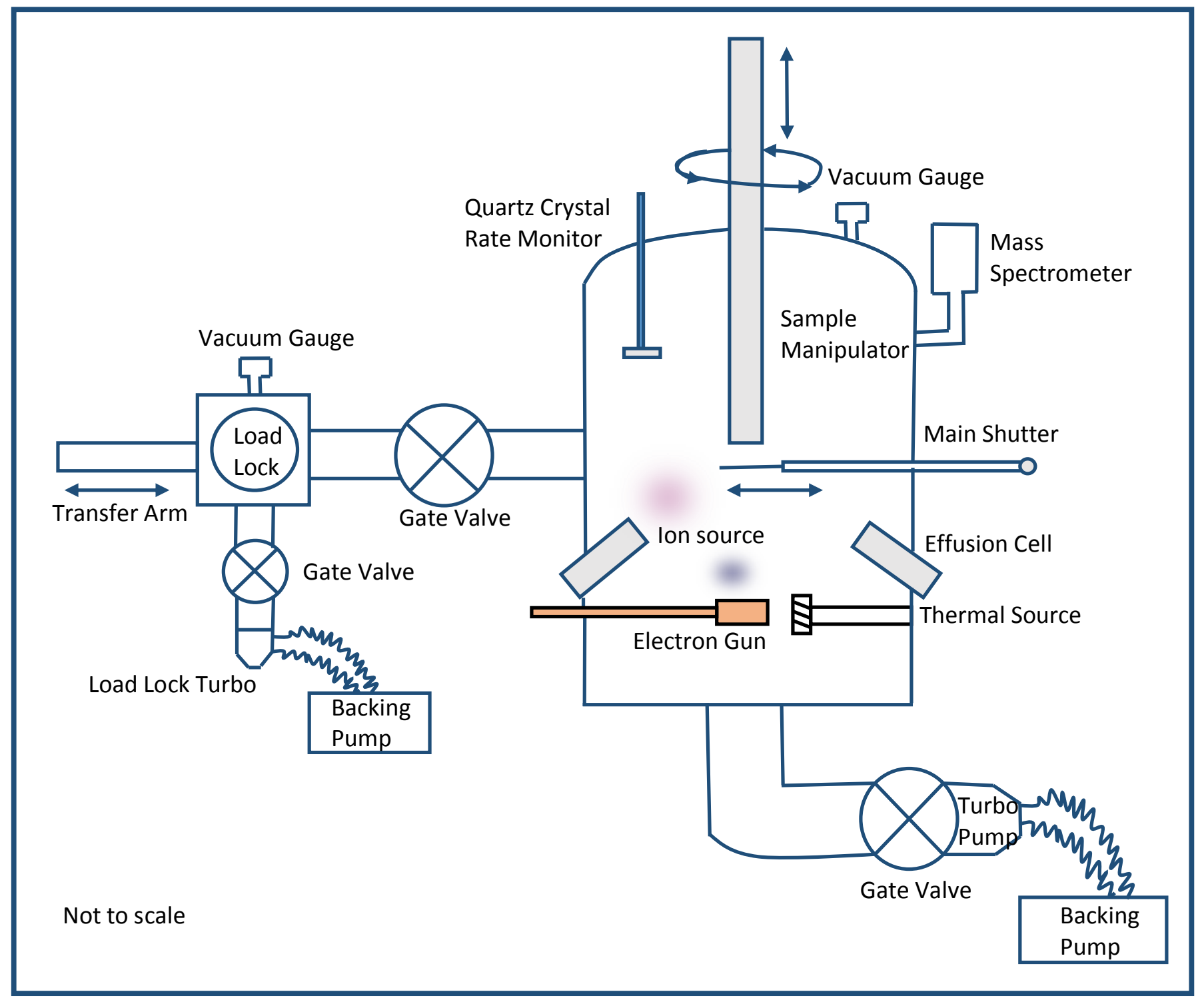

Figure 3.1: Schematic diagram of an ultra-high vacuum deposition chamber at thin film lab,

The Victoria University of Wellington, New Zealand.

This system consists of two chambers; one is known as the main chamber or growth chamber. This chamber is connected to a backing pump and a turbo pump to achieve a high vacuum $\left(10^{-7}\right.$ to $10^{-8}$ mbar). This chamber fitted with a substrate holder and a shutter. This shutter protects the substrate from unnecessary deposition during the outgassing process of RE metals and capping layer's materials. The other chamber is a load-lock chamber and it is also 
connected to backing and turbo pumps. A gate valve separates the growth chamber from the load lock chamber and maintains the different pressure in each chamber. The load lock part of the main chamber plays a crucial role of an airlock to allow transferring of samples in and out of the chamber without changing the pressure in the growth chamber. As a result, we can save time getting the required base pressure and avoid unnecessary baking of the system for each sample preparation.

A mass spectrometer was connected to the growth chamber and run by computer software. This is used to identify the different species of gas contained in the UHV chamber which helped us to achieve a better-quality sample. Inside the growth chamber, two quartz crystal microbalances were connected for monitoring the thickness of the films. The UHV chamber is equipped with a reflection high energy electron diffraction (RHEED) facility which allows monitoring of the surface structure of the substrate and film before and during the growth. Unfortunately, RHEED was down for the maintenance and not used while we grew the REN films. A heater was attached to the substrate holder for the high temperature sample growth. During the growth, the substrate holder rotates to ensure the film surface is uniform.

\subsection{Growth procedure}

The high purity gadolinium (Gd) and samarium (Sm) (99.9\% pure) were bought from the Kurt J. Lesker Company in the form of $3 \mathrm{~mm}-6 \mathrm{~mm}$ size pieces. Ingots of $\mathrm{Gd}$ and $\mathrm{Sm}$ were stored in mineral oil to prevent oxidization. Before placing them in the growth chamber, we cleaned them with either ethanol or isopropanol. The substrate also cleaned chemically in the sonicator bath using acetone. Later they were cleaned with nitrogen gas before mounting onto a substrate holder. The substrate holder was then installed into a load lock chamber prior to transfer into the main growth chamber. After installing the sample in the load lock chamber, we sealed and evacuated it using rotary and turbo pumps for several hours. We then transferred the sample into the growth chamber using a magnetically-coupled linear transfer arm as shown in Figure 3.1. Finally, the transfer arm is retracted and the gate valve between load-lock and growth chamber closed for achieving a lower pressure in the growth chamber.

Once the system is ready for the REN growth, we follow a standard practice, such as outgassing the rare-earth metals sources and capping layer materials at a high temperature. During this 
process, the substrate was protected by the shutter to prevent depositing the outgassing material on the substrate. This process expelled water or oxygen on the surface of the rare-earth metals and the capping layer materials. Generally, the RE metal and capping layer materials were slowly evaporated for approximately 15 minutes by varying the applied current. The remaining $\mathrm{O}_{2}$ molecules or $\mathrm{H}_{2} \mathrm{O}$ molecules were mostly pumped from the chamber before deposition began. This careful outgassing procedure significantly reduced the $\mathrm{O}_{2}$ pressure and other contamination inside the chamber.

In order to grow a REN thin film, we evaporated RE metal in the presence of high purity $\mathrm{N}_{2}$ gas. The flow of $\mathrm{N}_{2}$ gas was controlled by a mass flow controller. For the thermal evaporation technique, the electrode current was increased gradually. As a result, the temperature of the RE metal increased at a constant rate. When the growth pressure $\left(10^{-4}\right.$ to $10^{-5}$ mbar $)$ became stable, the shutter was opened to expose the substrate to the evaporated RE metals.

The two main sources of evaporation available in the growth chamber are thermal evaporator and electron beam evaporation. We followed different heating methods for $\mathrm{Gd}$ and $\mathrm{Sm}$. Electron beam evaporation was used to evaporate Gd which shows a high melting point of $1312^{\circ} \mathrm{C}$ while the thermal evaporator was employed to evaporate $\mathrm{Sm}$ as it exhibits a lower melting point of $1072^{\circ} \mathrm{C}$ and much higher vapour pressure at lower temperatures.

For the electron beam evaporation process, the rare-earth nitride and capping layer materials were put directly in the four pockets available inside the growth chamber. The electron gun emits a high energy electron beam that is directed by magnetic fields to focus in the centre of the desired evaporating material. This process is mainly used for samples with high melting points, such as gadolinium. Apart from using the electron beam evaporation we also use resistively heated thermal source for RENs growths. In this case, REN and capping layer materials are kept in crucibles which are surrounded by a tungsten heating coils and covered by shutters. In our thin film deposition lab, Sm and Ga are more commonly kept in two boron nitride (which has a high melting point) crucibles for the thermal evaporation process.

The deposited rare-earth metal reacted with the nitrogen to form a nitride film on the substrate. The growth rate and the stoichiometry of the films were heavily dependent on the ratio between the rare-earth flux and the nitrogen flux incident on the substrate. The typical growth rate for RE metal was about $\sim 0.1 \mathrm{~nm} \mathrm{~s}^{-1}$. The growth rate $(\AA / \mathrm{s})$ and thickness of the film was monitored in-situ by one of two quartz crystal microbalances. 
After achieving the desired thickness for a REN film, we used the thermal evaporation technique to grow the capping layer. In our case, polycrystalline GdN and SmN films were capped by GaN. Description of the growth of GaN can be found in reference [157]. Once the growth process was completed, we closed the shutter, turned off the nitrogen supply, stopped the water flow and secured all connections. Before removing the sample from the chamber, we allowed it to cool down to room temperature. Then we turned off the turbo pump of load lock chamber and waited around two hours for it to stop completely. Finally, prior to opening the load lock chamber, we shut the backing pump and ensured that all safety procedures were followed. Once the sample was removed from the chamber, it was quickly transferred to the desiccator to avoid oxidation.

\subsection{Growth procedure of epitaxial films in France}

This section presents the growth procedure of epitaxial GdN and $\mathrm{SmN}$ films grown by our collaborator in France.

The high purity gadolinium (Gd) and samarium $(\mathrm{Sm})$ (99.9\% pure) were bought from the American Elements Company in the form of rods of $5 \mathrm{~mm}$ diameter and $10 \mathrm{~mm}$ length. Rods of Gd and Sm were stored in dry nitrogen environment to prevent oxidization. We used AlN (0001) templates grown on silicon (111) substrates (2 inches) to reduce the lattice mismatch with depositing GdNs and SmNs. The other reason is to avoid the formation of silicides layers when we try to grow RENs directly on Si at high temperatures.

Before transferring the substrate to the UHV chamber it was cleaned properly. First it was deoxidized and passivated (with hydrogen terminations) by using a chemical wet etching of hydrofluoric acid (HF 5\% for 2 minutes). Then the substrate was rinsed using ultrapure water (18 M Ohms) and dried with nitrogen gas. After completing the cleaning process the substrate was mechanically mounted on molybdenum sample holder. The sample mounted in such a way to allow direct infrared heating of the substrate from a heater filament when inside the vacuum chamber.

After loading the sample in the introduction chamber, the chamber was pumped with an Alcatel Drytel 100 (oil free) pumping station composed of a diaphragm pump and a turbo pump. Once the introduction chamber reached to the desire pressure, the substrate was transferred to the outgassing chamber and perform the outgassing process at $200^{\circ} \mathrm{C}$ around one hour. In the MBE 32 Riber system, substrates are placed on a carriage trolley which can convey eight holders at 
one time. This trolley, which is pulled along rails by a chain manipulated from outside, can convey the substrate from the introduction chamber to the buffer chamber (or growth chamber). The outgassing chamber is located between the introduction and buffer chambers. The substrate was moved into the growth chamber by using a magnetically-coupled linear transfer rod once we completed the outgassing process. All the details of the growth procedure of AlN on silicon (111) substrates can be found in the article published by our collaborator [158]. The growth of $\mathrm{GdN}$ and $\mathrm{SmN}$ was performed at high temperature.

Basically we used solid sources for the metallic elements ( $\mathrm{Ga}$ and $\mathrm{Al})$ and ammonia $\left(\mathrm{NH}_{3}\right)$ as a nitrogen precursor for the growth of $\mathrm{GaN}$ (capping layer) and AlN (buffer layer). Contrary to the RENs growth, using molecular nitrogen $\left(\mathrm{N}_{2}\right)$ is not possible for $\mathrm{AlN}$. $\mathrm{NH}_{3}$ is thermally cracked to provide atomic nitrogen species for GaN and AlN instead. Typical growth temperatures for $\mathrm{GaN}$ and $\mathrm{AlN}$ are $800^{\circ} \mathrm{C}$ and $900^{\circ} \mathrm{C}$, respectively. However the lower growth temperature was used for the $\mathrm{GaN}$ capping layer because growing at $800^{\circ} \mathrm{C}$ leads to a columnar and not coalesced $\mathrm{GaN}$ layer. By decreasing the growth temperature to $650^{\circ} \mathrm{C}$, a compact $\mathrm{GaN}$ capping layer was achieved. Although the structural quality of GaN, assessed by X-ray diffraction, was decreased compared to the growth at $800^{\circ} \mathrm{C}$, due to an increase of the mosaicity. But this was not affected the RENs as the main goal of this layer was to cover the REN layer to prevent from the oxidation.

The Riber system needs to be cooled down after completing all growth process (RENs and Capping layers) and then we followed all standard procedure to transfer the sample from the growth chamber to the introduction chamber. Finally, the sample was taken out from the introduction chamber and put it in a desiccator. The following table shows a list of REN films which have been grown for this Ph.D. project. 
Table 3.1: Growth parameters for $\mathrm{GdN}$ and $\mathrm{SmN}$ films.

\begin{tabular}{|c|c|c|c|c|c|c|}
\hline Sample & $\begin{array}{c}\text { Nitrogen } \\
\text { Pressure } \\
\text { (Torr) }\end{array}$ & $\begin{array}{c}\text { Thickness } \\
\text { (nm) }\end{array}$ & $\begin{array}{c}\text { T}_{\text {Growth }} \\
\left({ }^{0} \mathrm{C}\right)\end{array}$ & $\begin{array}{c}\text { Growth } \\
\text { Rate (A/s) }\end{array}$ & Substrate & Capping layer \\
\hline Epitaxial GdN_HC & $2.7 \mathrm{E}-5$ & $120 \pm 10$ & 740 & $0.33 \mathrm{~A} / \mathrm{s}$ & $\begin{array}{l}\text { Si(111)/50nm - } \\
\text { AlN }\end{array}$ & $120 \pm 10 \mathrm{~nm} \mathrm{GaN}$ \\
\hline $\begin{array}{l}\text { Epitaxial GdN_HC } \\
\text { (For thermopower) }\end{array}$ & $2.7 \mathrm{E}-5$ & $120 \pm 10$ & 740 & $0.33 \mathrm{~A} / \mathrm{s}$ & GaN & $120 \pm 10 \mathrm{~nm} \mathrm{GaN}$ \\
\hline Epitaxial GdN_LC & $2.7 \mathrm{E}-5$ & $120 \pm 10$ & 650 & $0.33 \mathrm{~A} / \mathrm{s}$ & $\begin{array}{l}\text { Si(111)/50nm - } \\
\text { AlN }\end{array}$ & $120 \pm 10 \mathrm{~nm} \mathrm{GaN}$ \\
\hline Polycrystalline GdN & $9.5 \mathrm{E}-5$ & $135 \pm 15$ & RT & $0.5 \mathrm{~A} / \mathrm{s}$ & DSP Sapphire & $50 \pm 5 \mathrm{~nm} \mathrm{GaN}$ \\
\hline Epitaxial SmN & $3.0 \mathrm{E}-5$ & $90 \pm 10$ & 400 & $0.25 \mathrm{~A} / \mathrm{s}$ & YSZ & $110 \pm 10 \mathrm{~nm} \mathrm{GaN}$ \\
\hline Polycrystalline SmN & $4.9 \mathrm{E}-5$ & $50 \pm 5$ & RT & $0.3 \mathrm{~A} / \mathrm{s}$ & Glass & $100 \pm 10 \mathrm{~nm} \mathrm{AlN}$ \\
\hline
\end{tabular}

* HC stands for high carrier concentration and LC stands for low carrier concentration

\subsection{Structural and compositional characterization}

\subsubsection{X-ray diffraction $(\mathrm{XRD})$}

$\mathrm{X}$-ray diffraction (XRD) is a commonly used technique to characterise the structural properties of a material. X-rays are electromagnetic waves with associated wavelengths, or beams of photons with associated energies, which are generated by a cathode ray tube. They are filtered to produce monochromatic radiation, collimated form a beam, and are directed towards a sample [128, 159].

When a monochromatic X-ray beam irradiates the sample, part of the beam is transmitted, part is absorbed by the sample, part is refracted and scattered, and part is diffracted. The interaction of the incident rays with the sample produces constructive interference (and a diffracted ray). The detector measures the diffraction pattern and stores the information in the particular XRD - software. The diffraction pattern obtained includes the position (angles) and the intensities of 
the diffracted beam and carries information about the sample. The possible diffraction directions of the lattice can be achieved by doing $\theta-2 \theta$ scanning. The position of the diffracted peaks provides insight into the atomic arrangement of the sample (unit cell size or lattice parameters and crystal structure). When certain requirements are met, scattered X-rays interfere constructively to produce the diffraction pattern, and we determine the distance between the planes of the atoms by applying Bragg's law:

$$
d=\frac{n \lambda}{2 \sin \theta}
$$

Where the integer $n$ is the order of the diffracted beam.

$d$ - Distance between adjacent planes of atoms (the d-spacing) which is measured in $\AA$.

$\lambda$ - Wavelength of the incident X-ray beam and

$\theta$ - Angle between the incident beam and the normal to the plane.

The lattice parameter $(a)$ of a cubic structure crystal can be calculated using

$$
a^{2}=d_{h k l}^{2}\left(h^{2}+k^{2}+l^{2}\right)
$$

where $d_{h k l}$ is the inter-planar distance for the plane described by Miller indices $h, k, l$.

All these values (lattice parameter, $d$-spacing, miller indices) generally compare to a standard diffraction pattern database from the International Centre for Diffraction Data (ICDD). By comparing the peaks appearing in the XRD pattern with the ICDD database, the crystal structure of the material being investigated can be identified.

In addition, the average crystallite size of the material is also determined by using Debye Scherrer formula

$$
D=\frac{k \lambda}{\beta \cos \theta}
$$

where $D$ is the average crystallite size, $k$ is the shape factor (generally taken as $\sim 0.9$ ) and $\beta$ is the full width at half maxima (FWHM) of a peak located at an angle $\theta$.

Our X-ray diffraction measurements were carried out using a panalytical (X'Pert-PRO) diffractometer equipped with a $\mathrm{Cu} \mathrm{K \alpha}$ radiation source operating at $30 \mathrm{kV}$ and $30 \mathrm{~mA}$. The 
spectra were collected for $2 \theta=20^{\circ}-80^{\circ}$ with a total scanning time of 20 - 25 minutes for each sample. XRD patterns of REN thin films were identified with the help of X'Pert high score plus software supplied with the instrument. The measurement was performed at the Victoria University of Wellington.

\subsubsection{XRD results}

This section describes the structural characterisation results of a $\mathrm{GdN}$ film grown at room temperature (polycrystalline) by me at the Victoria University of Wellington and other epitaxial GdN and SmN films grown at high temperature by Valbonne, France group. Figures 3.2, 3.3 and 3.4 show XRD spectra of epitaxial GdN_HC film, polycrystalline GdN film and epitaxial SmN film, respectively. Epitaxial GdN_HC and GdN_LC films were grown on AlN template on a silicon substrate and polycrystalline GdN film was grown on a double side polished sapphire substrate. The SmN film was grown on yttria stabilized zirconia (YSZ) substrate. Substrates were chosen carefully after considering the requirement of experiments. The XRD result of epitaxial GdN_LC film was not available as the XRD system was down for the maintainance. All prominent peaks are labelled in XRD figures. The other small unlabelled peaks arise from the GaN capping layer and XRD sample holder.

The epitaxial GdN_HC film was grown at high temperature $\left(750^{\circ} \mathrm{C}\right)$ onto silicon (111) substrate coated with a $50 \mathrm{~nm}$ thick AlN template and the epitaxial $\mathrm{SmN}$ film was grown at $400^{\circ} \mathrm{C}$ onto YSZ substrate. Films grown in this way have been shown to be epitaxial quality before $[107,108]$. It was found that the GdN films are oriented along the (111) axis evident from main XRD peak at $2 \theta=31^{\circ}$. This is consistent with the previous literature reports where (111) was observed as a major peak in GdN [108]. 


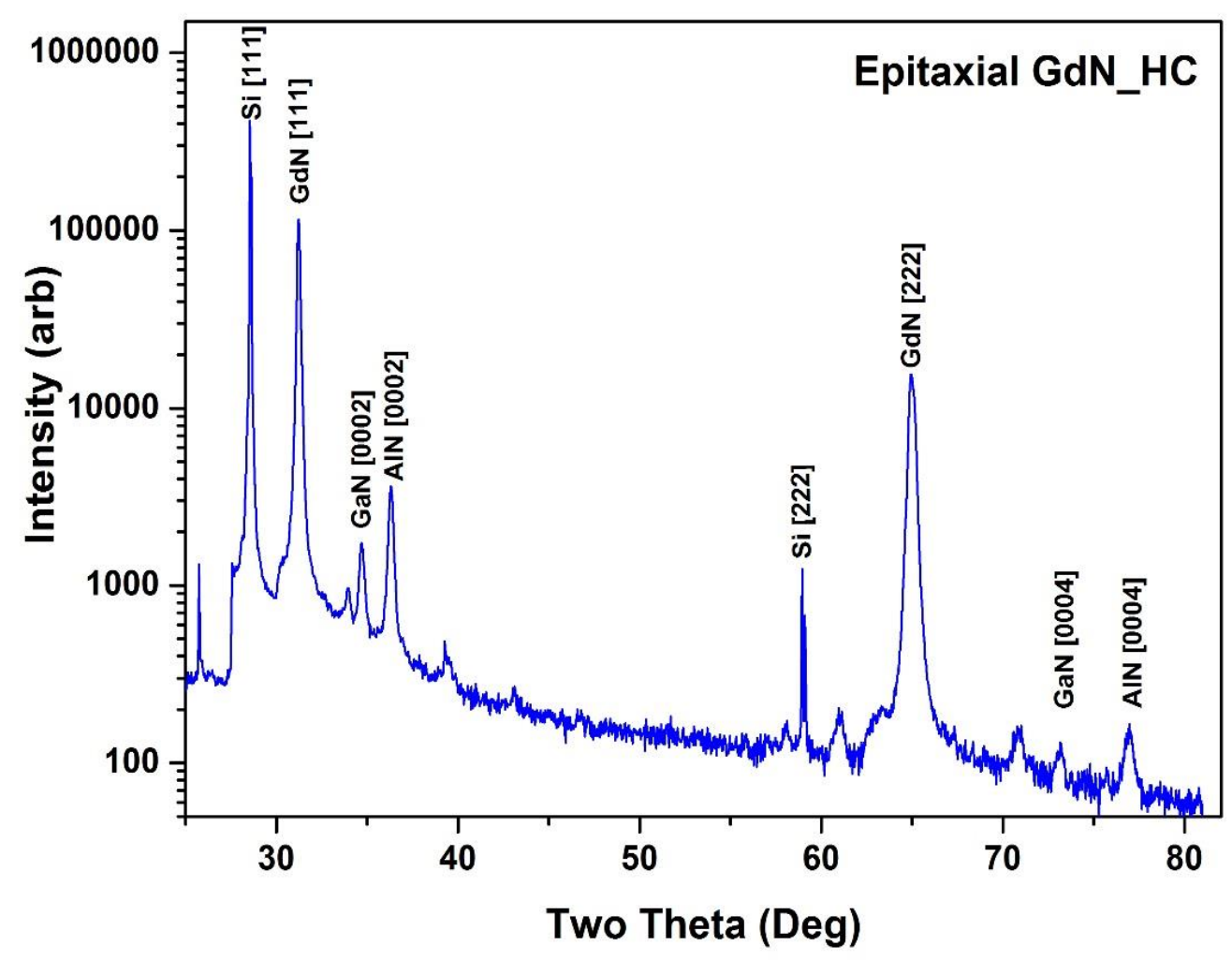

Figure 3.2: XRD scan of an epitaxial GdN film grown on an AlN template on a silicon substrate at high temperature. 


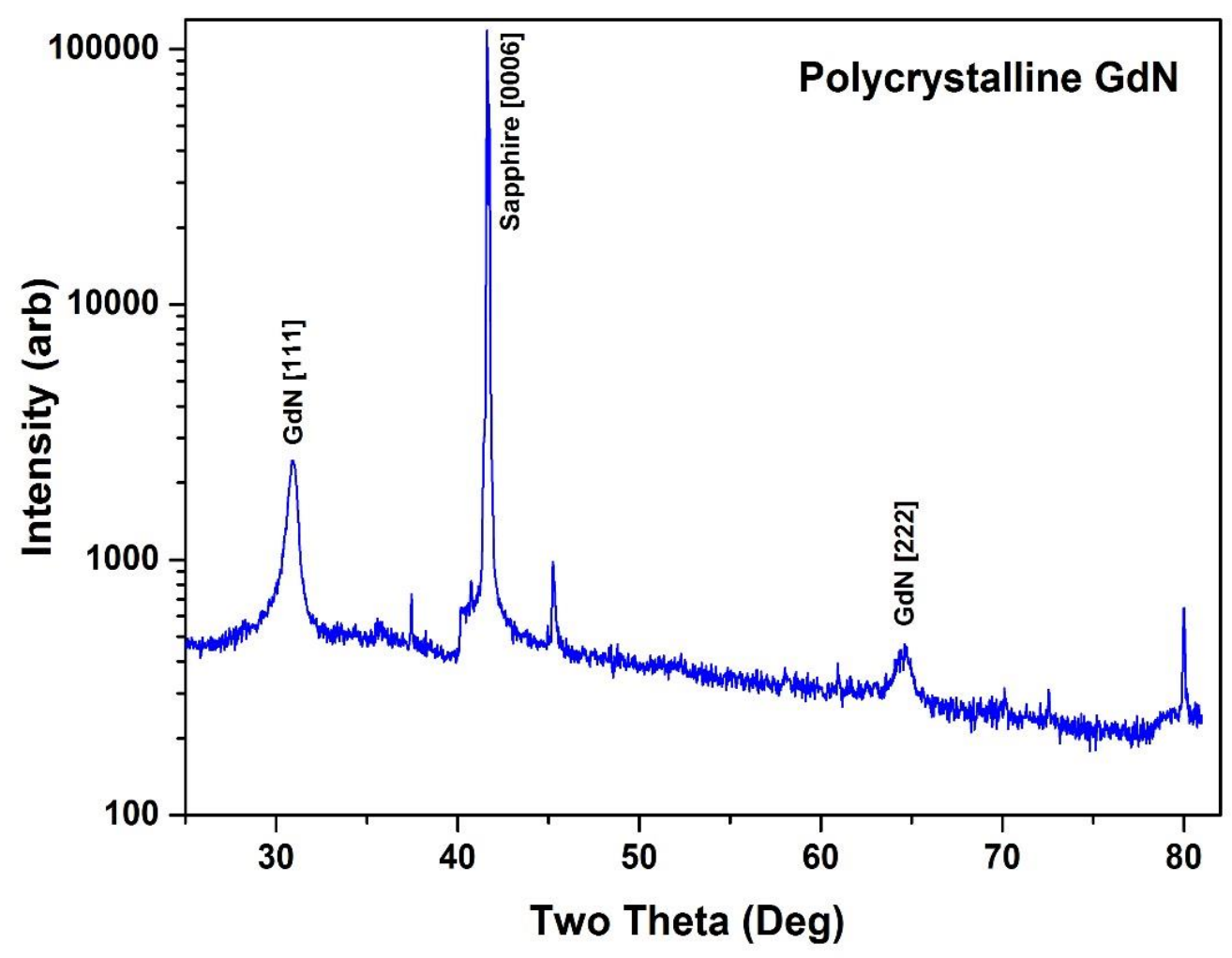

Figure 3.3: XRD scan of a polycrystalline GdN film grown on a sapphire substrate at room temperature.

The lattice constant of the $\mathrm{GdN}$ and $\mathrm{SmN}$ films were calculated from the diffraction pattern using Bragg's law. The lattice constants were found to be $4.99 \pm 0.5 \AA$ and $5.02 \pm 0.5 \AA$ for the epitaxial and the polycrystalline GdN films and $5.05 \pm 0.5 \AA$ for the epitaxial SmN film, respectively. For the SmN film we observed that substrate peak merged with the SmN peak which we often see while both peaks appear nearly the same position. Therefore extra care is required for calculating the lattice constant from the same peak. These measured lattice constants are close to the values reported in the literature [15, 24], which gives us confidence about the sample quality. The lattice constant can deviate from the standard value due to the presence of lattice mismatch with the substrate or intrinsic defects such as nitrogen vacancies.

The average crystallite size was extracted from the Scherrer formula using (111) peak in a $\theta$ $2 \theta$ scan and was found to be around $14 \mathrm{~nm}$ for the polycrystalline sample and have larger crystallite size $(\sim 28 \mathrm{~nm})$ for the epitaxial film. The epitaxial $\mathrm{SmN}$ film has been shown to have a crystallite size $(\sim 12 \mathrm{~nm})$. 


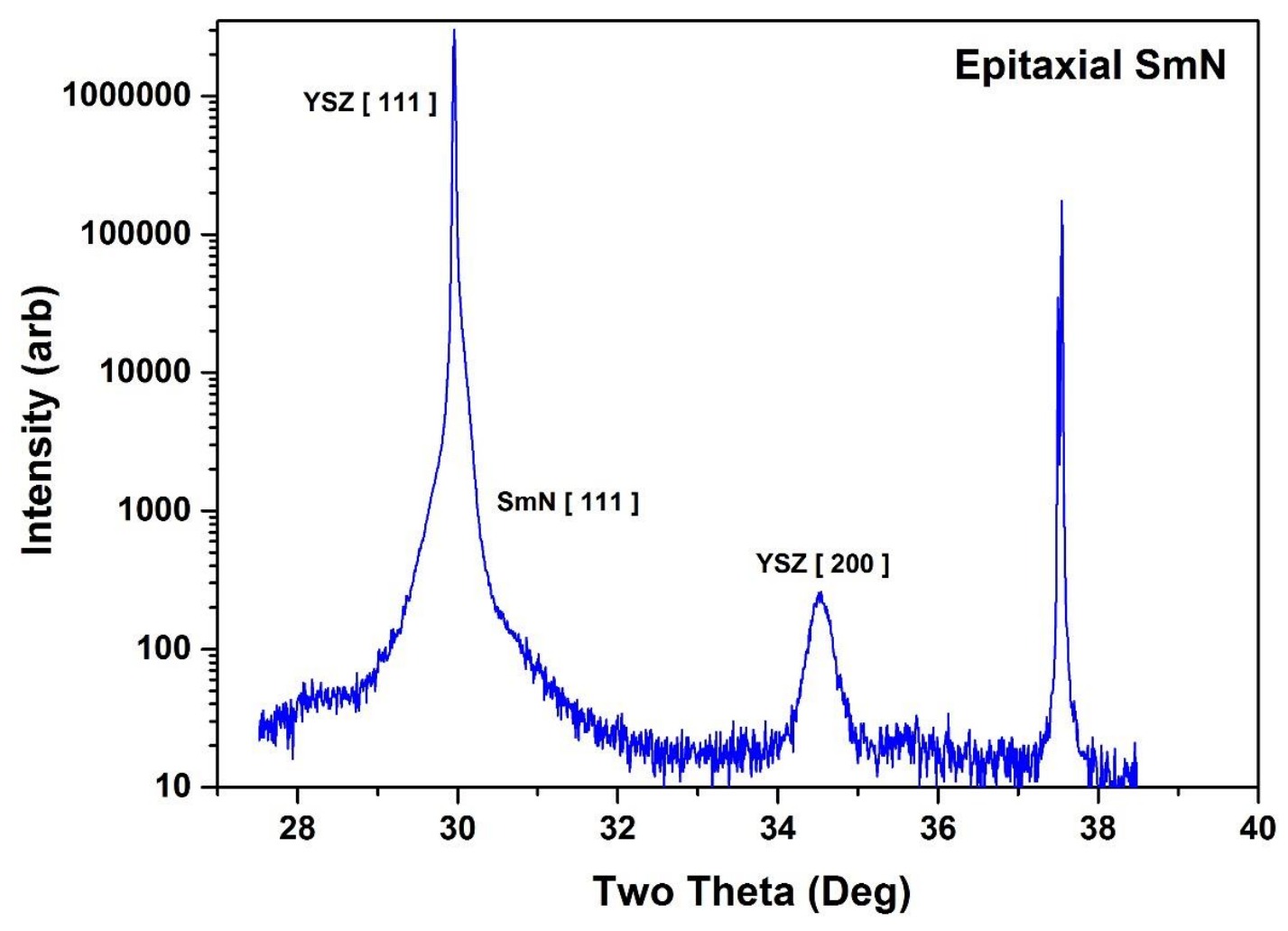

Figure 3.4: XRD scan of the epitaxial $\mathrm{SmN}$ film grown on an YSZ substrate at high temperature.

\subsubsection{Rutherford backscattering spectrometry (RBS)}

Rutherford backscattering spectrometry (RBS) is an analytical technique widely used to determine the elemental composition, depth profiling of individual elements, thickness calculation, impurity analysis, foreign atom location and to determine the crystalline quality of a thin film [160 - 163]. RBS technique was successfully developed between 1909 and 1914 by Geiger-Marsden to identify the structure of an atom. In 1911, Rutherford originally used the backscattering of alpha particles from a gold film to determine the fine structure of the atom and the experiment resulted in discovery of atomic nucleus. In 1957, Rubin et al. [161] first demonstrated that RBS could be used for materials analysis. The modern application of RBS is widespread in the field of material, nuclear, surface, geology, archaeology, biology and environmental sciences due to its versatile features. We used RBS to determine the elemental composition and the thickness of REN thin films. 
In our RBS experiments, the thickness of the REN films was obtained in terms of atom $\mathrm{cm}^{-2}$ and the elemental composition of the REN films was calculated from the RBS spectrum by using the Rutherford Universal Manipulation Program (RUMP) (simulation code developed in 1983 by M. Thompson at Cornell University, USA) [164]. The sample composition and thickness were derived in terms of areal density (number of atoms per unit area) in the layer. The experimental results contain 5-10\% uncertainty arising from statistical errors. RBS experiments were performed at the GNS Science, Gracefield with a 3 MV Van-de-Graaff accelerator.

\subsubsection{RBS result}

Figure 3.5 shows a typical RBS spectrum of the polycrystalline GdN film grown at room temperature on a $\mathrm{Al}_{2} \mathrm{O}_{3}$ substrate with $\mathrm{GaN}$ capping layer. In $\mathrm{RBS}$ spectrum the $\mathrm{X}$-axis is usually channel number which basically is the backscattered particle energy. The backscattered particle energy depends on mass of the elements as well as on the location of the particular atom in the film. The $\mathrm{Y}$-axis represents the cross-section of the elements which is proportional to $\mathrm{Z}^{2}$. Here $\mathrm{Z}$ is the atomic mass of elements which determines intensity of the signal.

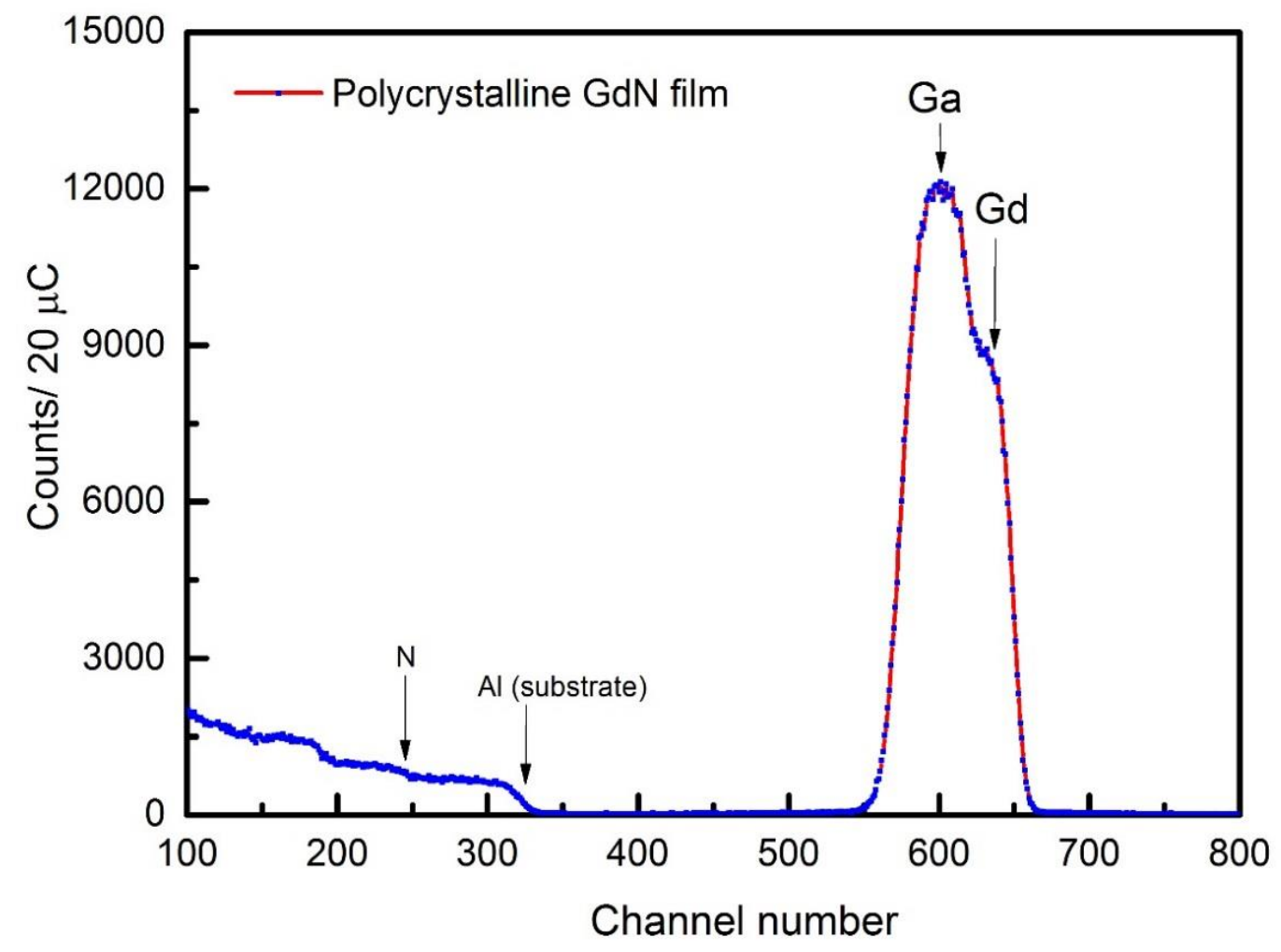

Figure 3.5: RBS spectrum of the polycrystalline GdN film. 
The RBS peak associated with Ga is seen near channel number 640. The Gd peak starts from channel number 660 and overlaps with the Ga peak. If $\mathrm{Gd}$ atoms were in the first layer (i.e. no GaN cap), the Gd signal would have appeared at slightly higher energy (e.g., around 700 channel number). Because Ga (atomic mass - 31) atoms are lighter than Gd (atomic mass - 64) and its backscattered particle energy is lower than $\mathrm{Gd}$.

The RBS peak from $\mathrm{Al}$ in the $\mathrm{Al}_{2} \mathrm{O}_{3}$ substrate appears from channel number 330 and extends to much lower channel number due to a thick layer. The $\mathrm{N}$ peak overlaps with the $\mathrm{Al}$ peak below channel number 250. N signal is much lower than Ga or Gd even though it sits on top of the $\mathrm{Al}$ signal from the substrate, even though, $\mathrm{N}$ concentration is similar to $\mathrm{Ga}$ or $\mathrm{Gd}$. For $\mathrm{N}$ the $Z^{2}$ is 49 whereas for $\mathrm{Gd}$ it is $4096\left(\mathrm{Z}^{2}=64 \times 64\right)$ which is way higher than 49 , hence we see much bigger signal.

The thickness of each layer was calculated in terms of atoms $/ \mathrm{cm}^{2}$ using RUMP code [164] and the total thickness of this film is calculated to be $135 \pm 15 \mathrm{~nm}$. The composition and thickness of the epitaxial films were obtained from scanning electron microscope measurements in Valbonne, France within the typical uncertainty of $\sim 10 \%$. 


\section{Chapter 4}

\section{Experimental details}

This chapter describes the experimental techniques used in the thesis. The working principles of the equipment used for sample analyses are highlighted in detail. In particular, emphasis has been given to the study of transport and the thermoelectric properties of rare-earth nitrides. The chapter has been structured as follows:

Section 4.1 is devoted to the equipment used for magnetic measurements to understand the nature of the magnetic ordering and the transition temperature of the materials. The Quantum Design superconducting quantum interference device (SQUID) magnetometer at the Robinson Research Institute (RRI), Gracefield has been used to perform the magnetic measurements of REN films. The temperature dependent magnetic measurements were performed between $2 \mathrm{~K}$ and $300 \mathrm{~K}$ and the field dependent measurements were performed between $-6 \mathrm{~T}$ and $+6 \mathrm{~T}$.

Section 4.2 describes the charge transport measurement which contains a few subsections. The contact deposition, temperature dependent resistivity, the magnetoresistance and the Hall effect measurement process are discussed in 4.2.1, 4.2.2, 4.2.3 and 4.2.4 subsections, respectively. All experiments were conducted at RRI. Finally, the associated hardware, experimental set-up and process for the thermopower measurement are described fully in the charge/heat transport Section 4.3. This experiment was carried out at the low temperature lab in the Victoria University of Wellington (VUW), New Zealand. 


\subsection{Magnetic measurement: SQUID}

The magnetic properties of REN samples were measured by a magnetic property measurement system (MPMS) from Quantum Design, which utilises a superconducting quantum interference device (SQUID) [165]. The SQUID is a very sensitive magnetometer and can measure a very small magnetic field ( $\left.\sim 10^{-21} \mathrm{Oe}\right)$ at cryogenic temperatures [166]. The higher sensitivity of this device is useful to determine the magnetic properties of a sample exhibiting a very small magnetization.

To carry out the measurements, samples were inserted into a nonmagnetic plastic straw and then transferred to the SQUID dewar with the help of a sample transfer rod. The plastic straw was used to avoid any stray magnetic moments. It is also recommended to use plastic or brass tweezers instead of using steel tweezers during the sample mounting process. This is because steel tweezers may transfer magnetic impurities to the sample which could significantly interfere with the measurement. In addition, the samples needed to be located in the centre of the SQUID measurement coils to obtain a better signal. Once the sample was installed properly we performed a measurement at room temperature by applying a magnetic field of $1 \mathrm{~T}$ to allow the software to centre the sample. The sample was moved up and down through the coil and the SQUID signal was converted into a sample magnetisation. We performed many scans for a particular measurement and counted the average scans to minimize error.

Two types of magnetic measurement as a function of temperature were mainly carried out for our REN samples. To observe the magnetic behaviour of a REN sample we performed zero field cooled (ZFC) and field cooled (FC) measurements. In the ZFC measurement, the sample was first cooled by sweeping the temperature between $300 \mathrm{~K}$ and $2 \mathrm{~K}$, without an applied magnetic field. Then the sample's temperature was increased from $2 \mathrm{~K}$ to $300 \mathrm{~K}$ in the presence of a small magnetic field and the magnetic moment of the REN sample was recorded as a function of temperature. For a field cooled (FC) measurement, the magnetic moment was recorded while the sample was cooled down to $2 \mathrm{~K}$ in the presence of a magnetic field.

The other type of measurement was magnetic field dependent. In order to perform this measurement at a given temperature (e.g., $5 \mathrm{~K}, 10 \mathrm{~K}, 20 \mathrm{~K}, 30 \mathrm{~K}$ ), the applied magnetic field was swept in the whole range from $-6 \mathrm{~T}$ to $+6 \mathrm{~T}$ and the magnetic response of the sample (hysteresis loop) was recorded as a function of the applied magnetic field. Additional information can be revealed from this measurement, such as the degree of magnetisation of the 
sample when the applied field was reduced to zero (the remanence), and how easily the magnetisation of the sample can be reversed (the coercive field). All REN films were measured with the applied magnetic field in the direction of the plane of the film (or in-plane field measurements).

The total magnetic moment of the REN sample was measured by the SQUID in electromagnetic units (emu). In the CGS units, the magnetisation or magnetic moment per unit volume of the sample is often mentioned in emu/ $\mathrm{cm}^{3}$. The other common unit is Bohr magneton per magnetic ion $\left(\mu_{\mathrm{B}} /\right.$ ion), which generally we use to analyse our REN samples. The Bohr magneton is approximately the magnetic dipole moment of a single electron and this unit is more easily compared with the number of electrons contributing to the magnetic moment. The magnetization/ unit volume of the sample in terms of Bohr magneton is given by,

$$
\mathrm{M}\left(\# \mu_{\mathrm{B}}\right)=\frac{m[e m u]}{\mu_{B}} / \mathrm{RE} \text { ions }
$$

where, $\mu_{\mathrm{B}}=9.274 \times 10^{-21} \mathrm{emu}$ and $m$ is the magnetic moment of the sample measured in CGS units from SQUID magnetometer. In order to calculate this we need to know the volume of the sample, which is the largest source of uncertainty in the results.

\subsection{Charge transport measurement}

In this section we will discuss various transport measurements, such as resistivity, magnetoresistance, Hall effect and thermopower measurements. Charge transport measurements of our REN samples as a function of temperature and field were performed mostly between $3 \mathrm{~K}$ and $300 \mathrm{~K}$. Before we discuss briefly each of these measurements, we will shed light on the electrical contact deposition which plays an important role for measuring the transport properties of rare-earth nitride films. Especially, significant attention should be taken with depositing reliable contacts which will work at low temperatures. 


\subsubsection{Electrical contact deposition}

For transport measurements of the sample, it is necessary to make electrical contacts on the REN samples. However, it is challenging to directly contact the REN films due to their propensity to react with oxygen, requiring a protective capping layer. There are a few methods described below to make electrical contact to the REN film even when coated with an insulating capping layer.

Firstly, electrical contacts can be deposited before substrates are loaded into the chamber for rare-earth nitride growth, referred to as pre-deposited or bottom contacts. This process is a bit complicated and required a special mask, substrate holder and thermal evaporator chamber. In this case, four electrical contacts (generally 70 - $100 \mathrm{~nm} \mathrm{Au} \mathrm{-} \mathrm{Ag} \mathrm{contacts)} \mathrm{were} \mathrm{grown} \mathrm{in} \mathrm{the}$ four corners of the square (usually $1 \mathrm{~cm}$ x $1 \mathrm{~cm}$ ) substrate using an Angstrom Engineering evaporator. The contacts are partially covered by a mask and the REN and capping layer are deposited on the substrate in the MBE chamber.

For charge transport measurements, electrical contact could then be made to the exposed predeposited contact using a small amount of silver paste or indium to connect thin $\mathrm{Cu}$ wires.

The second process is more straightforward and it allows us to perform the charge and heat transport measurements of REN samples without growing pre-deposited contacts on the substrate. For this method, we used silver paste or pressed indium to make contact directly on the top of the REN film through the resistive capping layer. Provided the capping layer is thin enough and not overly insulating, the probing current passes vertically through the capping layer and horizontally along the sample length through the REN layer. 


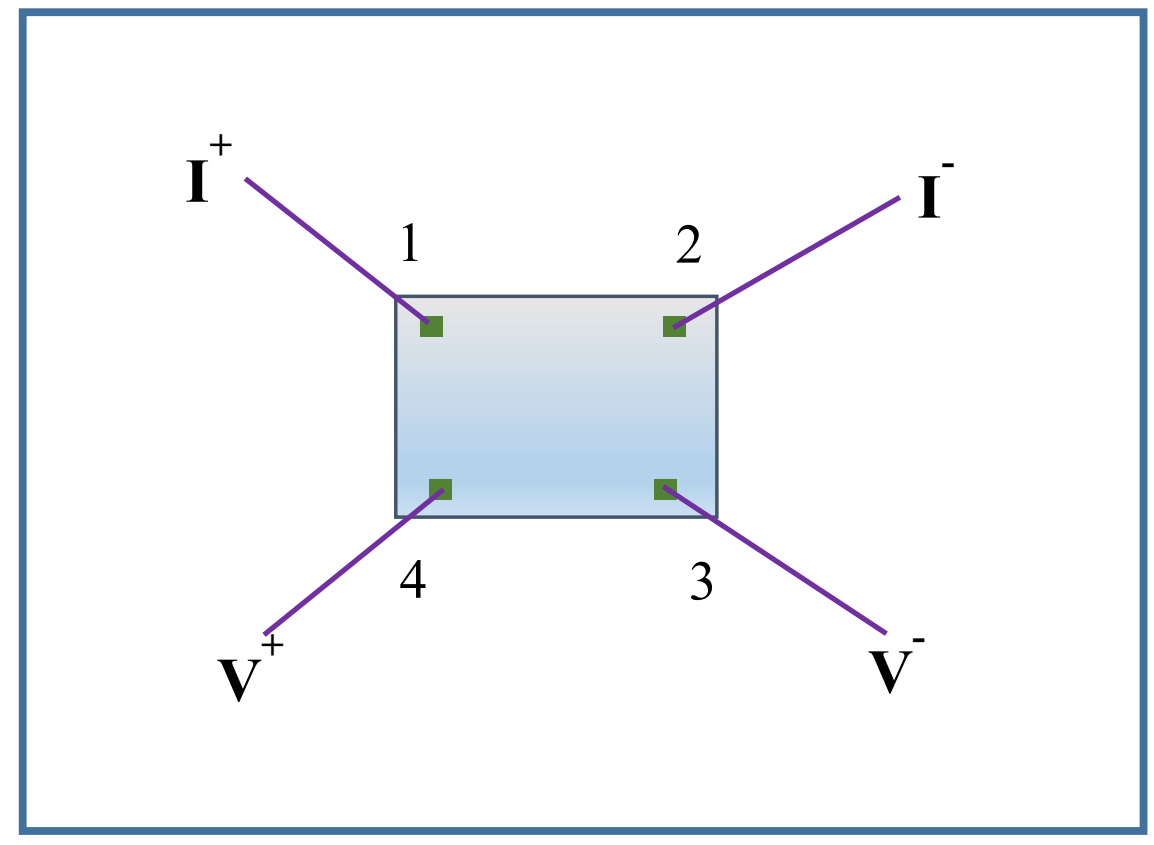

Figure 4.1: Illustration of wire connection for resistivity measurement.

Pre-deposited contacts are generally preferred and were used wherever possible for the samples grown in our MBE lab at Victoria University of Wellington (VUW). However the majority of samples presented in this thesis were epitaxial films grown by our collaborator for which predeposited contacts could not be used. Top contacts through the capping layer was thus unavoidable for these samples. An advantage of using pressed indium is that the contacts are far less likely to fail when the system is cooled. In contrast, silver paint has a tendency to crack at low temperature because of a mechanical failure, causing the contacts to fail.

The van der Pauw geometry for making contacts is used due to the following: An applied current $(I)$ is passed through the two terminals $(1,2)$ and the potential difference is measured across the remaining two terminals $(3,4)$ as shown in the Figure 4.1. The very high impedance value of the voltmeter means that the voltage leads draw very little current. Generally, there is negligible or no current conduction through the voltage terminals and the current is primarily conducted through the sample. This method eliminates the lead and contact resistances. Hence both the current and the voltage drop across the sample can be measured to a high degree of accuracy. 


\subsubsection{Physical property measurement system (PPMS)}

A Quantum Design made physical property measurement system (PPMS) is a commonly used commercial instrument for performing charge transport and magnetic measurements [167]. This flexible system offers a wide range of measurements including resistivity, magnetoresistance and Hall effect measurements [167]. The magnetoresistance and the Hall effect measurements can be done in the presence of applied magnetic fields between - $9 \mathrm{~T}$ and $+9 \mathrm{~T}$ and at temperatures ranging from $1.9 \mathrm{~K}$ to $400 \mathrm{~K}$. In addition, magnetic measurements can be performed in the same mentioned magnetic fields and temperature regions. Initially we checked all the parameters for a given experiment and then after a certain period of time the experiment performed automatically by executing a sequence file. In the next section we first explain the resistivity and magnetoresistance measurements followed by the description of the Hall effect measurement.

\subsubsection{Resistivity and magnetoresistance measurements}

The resistivity measurement of REN samples in the PPMS system is more straightforward than other transport measurements performed in this thesis. After making the contacts onto the REN sample, the sample was mounted on a resistance bridge sample holder board (Figure 4.2) (often called PPMS PUCK) to be used with the PPMS horizontal rotator. The reason for using the horizontal rotator is that the sample can be rotated $\left(180^{\circ}\right)$ around an axis perpendicular to the magnetic field of the longitudinal PPMS magnet to get the preferred orientation of the applied magnetic field and current during magnetoresistance measurements. The sample needs to be cut properly to fit within the sample space of the board as seen in Figure 4.2. The sample holder board was then attached to the horizontal rotator probe and inserted into the PPMS sample chamber to do the measurements. 


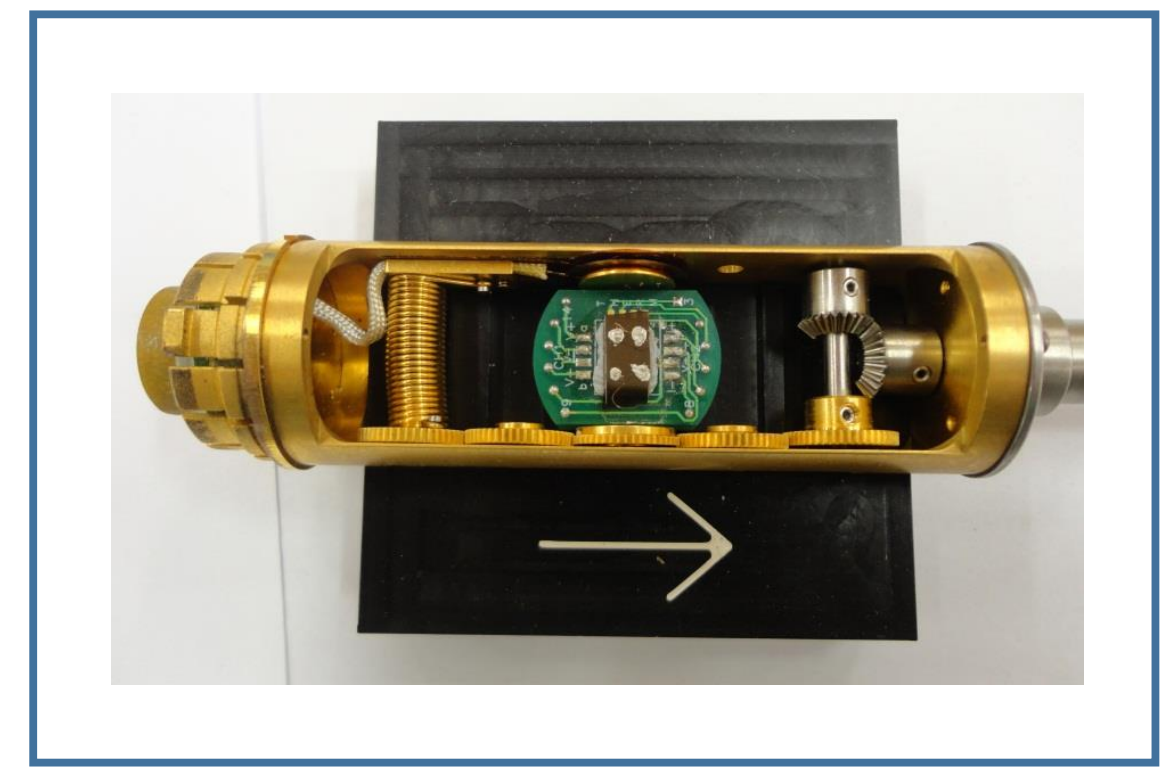

Figure 4 2: REN sample was mounted on a resistance bridge sample holder board inside the PPMS rotator probe for four-wire resistance measurements.

After transferring the REN sample inside the PPMS chamber, we sealed the chamber and then turned on the pump to achieve the desired pressure. Once the chamber achieved the required pressure, the rotator thermometer for temperature control was activated, and the appropriate configuration files were sent to the PPMS system to initialise the horizontal rotator. The rotator thermometer was deactivated on completion of the experiment by the automatic sequence setting. All instructions for the desired measurement were written in a sequence file and then the sequence file was executed using MultiVu software. The resistivity measurements were typically done from $3 \mathrm{~K}$ up to $300 \mathrm{~K}$, without an applied magnetic field and the uncertainty of this measurement mainly comes from the thickness of the sample.

When a resistivity measurement is performed in the presence of an external magnetic field, it is called a magnetoresistance (MR) measurement. In this experiment, we record the change in electrical resistivity of a material at different temperatures in the presence of an external magnetic field. In-plane and out-of-plane magnetoresistance measurements were performed with the horizontal rotator was set to the $0^{\circ}$ and $90^{\circ}$ positions, respectively. The arrow signs in Figure 4.2 indicates the direction of the sample rotation. The magnetoresistance of all our REN samples was carried out by sweeping the magnetic field from $-8 \mathrm{~T}$ to $+8 \mathrm{~T}$ at different set temperatures between $3 \mathrm{~K}$ and $300 \mathrm{~K}$, at the RRI, Gracefield. 


\subsubsection{Hall effect measurement}

Hall effect measurements were first used experimentally in the semiconductor industry to characterize bulk silicon [168]. After which the experiment did not achieve much attention until complicated problems arose in the semiconductor industry. A Hall effect measurement is generally used to determine electrical transport parameters of the materials. Among these parameters, the Hall voltage $\left(V_{H}\right)$, the Hall co-efficient $\left(R_{H}\right)$, the mobility of major charge carriers, carrier density and carrier type ( $\mathrm{N}$ or $\mathrm{P}$ ) are most important.

Our REN samples were grown with different parameters and doping levels (nitrogen vacancy concentration); these parameters can vary the carrier concentration of the REN samples. Using the Hall effect measurement we successfully obtained the type of carriers, carrier density and carrier mobility in each REN sample. We used the commercially available physical property measurement system (PPMS) [169] at the RRI, Gracefield to perform this experiment. Again the van der Pauw geometry was followed to make the contacts on the sample.

Four small contacts were deposited in the four corners of the sample (Figure 4.1). Two diagonal contacts (contact 1 and contact 3) were used for the current source. The Hall voltage was measured perpendicular to the current between the remaining two diagonal contacts (contact 2 and contact 4) at a finite magnetic field and zero magnetic field. A REN sample was attached to the horizontal rotator to transfer inside the PPMS chamber following the same procedure as in the case of resistivity and MR measurements (see the Section 4.2.3). The horizontal rotator probe was utilised to rotate the sample in two different directions to eliminate the effect of a magnetoresistive contribution to the Hall voltage. The first set of data was recorded at $0^{\circ}$ position and then the sample was rotated by $180^{\circ}$ to get the second set of data. The Hall voltage was calculated by subtracting the $180^{\circ}$ data from the $0^{\circ}$ data and then dividing by 2 . The concept behind this process is quite interesting and discussed briefly in the following paragraph.

It is quite difficult to deposit the perfectly aligned contacts when we determine the Hall coefficient. In practice, there will always be some imperfect alignment of the contacts, even if we use careful patterning of the sample into a Hall bar. If the material is non-magnetic, we would measure a Hall voltage plus an extra offset voltage. The offset voltage is a resistive one. The Hall voltage would vary with the field, and the offset voltage would be independent 
of field. If the contacts were perfectly aligned, there should be no voltage in zero field, so we could simply subtract the zero-field voltage to get the Hall voltage vs field.

But the thing becomes more complicated for a magnetic material due to the contribution of magnetoresistance in the Hall voltage result. In this case the offset voltage will also vary with the field, and we can no longer subtract a constant to get the Hall voltage. However, we know that the magnetoresistance is symmetric around zero field, i.e. $M R(+H)=M R(-H)$, whereas the Hall voltage is antisymmetric around zero field, i.e. $V_{\text {Hall }}(+H)=-V_{\text {Hall }}(-H)$. That means we can cancel out the magnetoresistance by measuring the voltage with the field in the positive direction, and then subtracting the voltage measured with the field in the negative direction. Let us consider that an ideal Hall effect measurement in the van der Pauw geometry, measuring the Hall voltage in a $0^{\circ}$ orientation (i.e. the field pointing up, out-of-plane of the film) and then $180^{\circ}$ orientation (i.e. the field pointing down, out-of-plane of the film).

So we could write:

$$
V_{0}=V_{\text {Hall }}+V_{M R}
$$

and then in $180^{\circ}$ the sign of $V_{\text {Hall }}$ is reversed,

$$
V_{180}=-V_{\text {Hall }}+V_{M R}
$$

So by subtracting one from the other, this removes the $V_{M R}$ part and leaving only $2 \times V_{\text {Hall }}$, i.e. $V_{0}-V_{180}=\left(V_{M R}-V_{M R}\right)+2 V_{\text {Hall }}=2 V_{\text {Hall }}$

Now we could write the Hall voltage is given by,

$$
V_{\text {Hall }}=\frac{\left(V_{0}-V_{180}\right)}{2}
$$

The Hall voltage as a function of a magnetic field was measured at different temperatures from $5 \mathrm{~K}$ to $300 \mathrm{~K}$ at the RRI, Gracefield. From the experimental data we work out the Hall coefficient, carrier concentration and mobility of the REN films to understand their conducting nature. 
The uncertainty of this measurement mainly arises from the sample thickness. We use it to calculate the carrier concentration and put the uncertainty bar in the carrier concentration data. The other thing is how accurately we estimate the Hall resistance slope from the experimental data. One could take the best fit and least fit of the slope and then consider the average value of the slope to estimate the carrier concentration. It is to be noted that sometimes we do see the noisy Hall effect data. This is could be related to the contacts issue or associated with the resistive nature of the sample. Maybe one or more contacts wires were not connected properly to the sample which therefore influence the magnitude of the Hall voltage. We also see the noisy data if samples become too resistive and the offset voltage is found to be much higher than the sample Hall voltage. In the results chapters (Chapter $5-8$ ) we have shown the temperature dependent Hall effect results of epitaxial GdN and SmN films whereas only the room temperature Hall effect measurement was only possible for the resistive polycrystalline GdN film. In addition it is also seen that the Hall effect data of relatively high resistive epitaxial GdN film is more noisy than the other less resistive epitaxial GdN film.

\subsection{Charge/heat transport measurement (Thermopower measurement)}

One of the interesting transport properties commonly used to understand electron transport is the thermoelectric power. In Section 2.3 we already discussed the theory and the importance of thermopower measurement of REN samples. In this section, the experimental details of thermopower are described in detail, beginning with a low temperature cryostat in which the REN samples were mounted and the measurements made between temperatures of $5 \mathrm{~K}$ and $300 \mathrm{~K}$. 


\subsubsection{Experimental apparatus}

The following experimental apparatus was used for the thermopower measurements:

i. $\quad \mathrm{SHI}-4 \mathrm{H}$ cryostat from Janis Research Co, Inc. Wilmington, USA

ii. Compressor - CSW - 71 D (SHI - Sumitomo Heavy Industries Ltd, Japan)

iii. BOC Edwards backing and turbo pumps

iv. Lakeshore 340 temperature controller

v. Lakeshore 101 current source

vi. Keithley 181 nanovoltmeter

vii. Yokogawa 7651 programmable DC source

viii. Two Tektronix DMM 40406 - 1/2 digit precision multimeter

ix. Two silicon diode thermometers from Lakeshore Cryotronics

X. Two copper blocks

xi. One heater made by the VUW mechanical workshop

xii. One plastic block

xiii. Manganin wire as reference leads

xiv. Constantan wire for the heater

xv. $\quad 0.05 \mathrm{~mm} \mathrm{Cu}$ wires

xvi. Heat sink cable and connector

xvii. One bobbin 


\subsubsection{Cryostat}

A SHI - 4H closed cycle cryostat from Janis Research Co., Inc, Wilmington, USA was used for our thermopower experiments. Figure 4.3 shows the schematic diagram of the closed cycle cryostat.

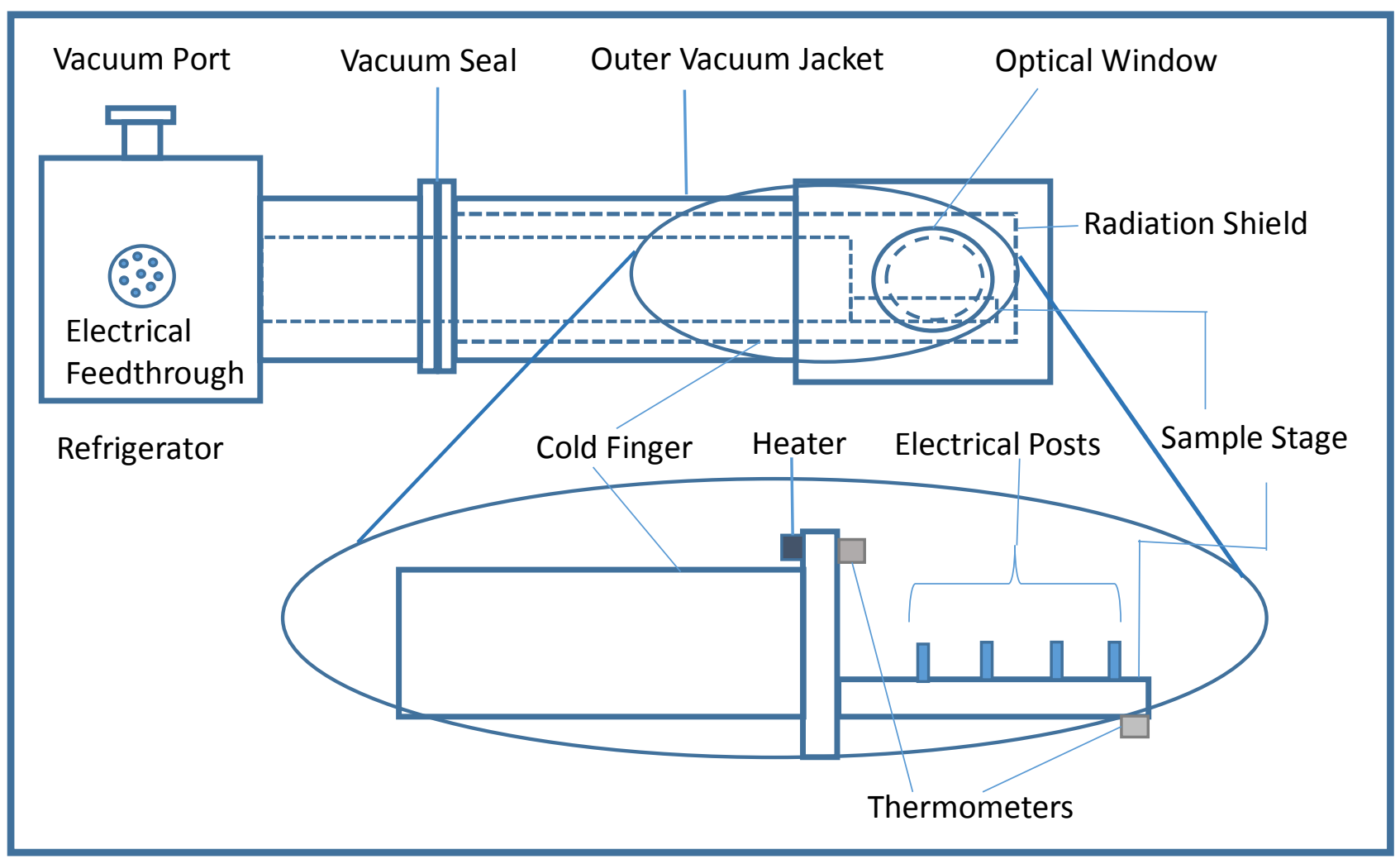

Figure 4.3: Schematic diagram of the closed cycle cryostat used for thermopower measurements.

The lower portion of the diagram is an expanded view of the sample stage.

The cryostat consists of a heat exchanger through which cold helium vapour was pumped. An external mechanical compressor extracted the warmer helium exhaust vapour, which was cooled and recycled. A medium size helium bottle was attached to the compressor to supply the compressor's helium, so that compressor can run continuously for an indefinite period without refilling. During the experiment the compressor required a continuous supply of cooling water to prevent it from overheating. 
The cryostat is constructed of stainless steel (outer jacket - aluminium) and copper. Stainless steel was used for the shields/casing to minimize thermal conduction, whereas copper was used for the cold stage. The main part of the cryostat was the metallic cold stage inside the vacuum chamber which was in thermal contact with the helium vapour chamber. The REN samples were thermally connected with the cold stage via a copper block. A Lakeshore 340 temperature controller was connected with the cryostat to control the temperature of the cold stage. The cryostat allows performing the experiment between $2 \mathrm{~K}$ to $300 \mathrm{~K}$.

\subsubsection{Wiring}

Electric wiring was required to set-up the thermopower rig inside the cryostat. The following experimental tools were utilised to build the thermopower set for the REN thin films:

i. Heater - The heater used to maintain the temperature of the hot end of the sample was made by us. One small bobbin $(0.7 \mathrm{~cm} \times 0.5 \mathrm{~cm})$ was wound with an insulated constantan wire and then wrapped in Teflon tape. The heater was attached to a small copper block and its resistance was 52 ohms.

ii. Silicon diode thermometers - Two silicon diode thermometers were attached to the two $\mathrm{Cu}$ blocks and required a minimum current of $10 \mu \mathrm{A}$ to operate. Figure 4.4 shows the schematic diagram of the DT - 470 - CU silicon diode thermometer used for the thermopower rig.

iii. Thermopower rig - Two Cu blocks made in our mechanical workshop were used to build the rig. The dimension of these two Cu blocks was $(0.8 \mathrm{~cm} \times 1.1 \mathrm{~cm} \times 1.1 \mathrm{~cm})$ and $(0.8 \mathrm{~cm} \times 0.8 \mathrm{~cm} \times 1.1 \mathrm{~cm})$. The smaller and the bigger sized blocks were used for the hotter and the colder side of the sample respectively. Figure 4.5 shows the block diagram of the thermopower rig developed for this thesis.

iv. Reference lead - Two manganin wires of equal length were taken and passed through a bobbin to minimise the spurious thermal voltage (see Section 4.3.6. Page number 81 for a further explanation). 


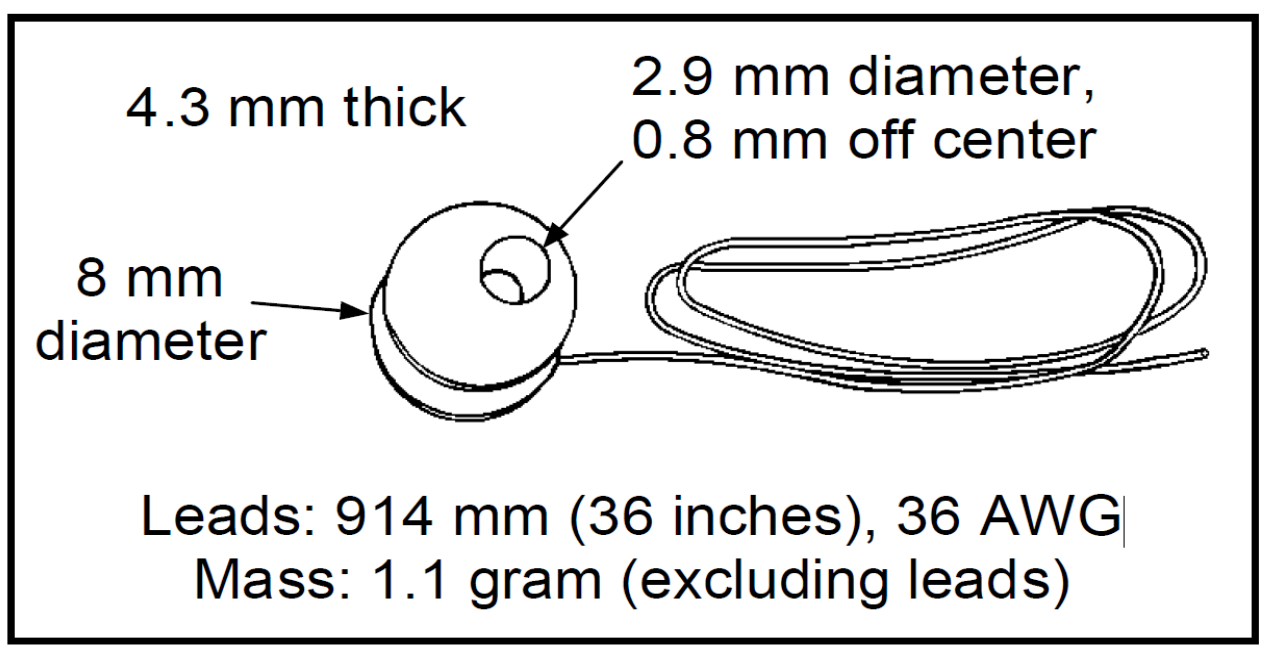

Figure 4.4: DT - 470 - CU silicon diode thermometer [Adapted from 170].

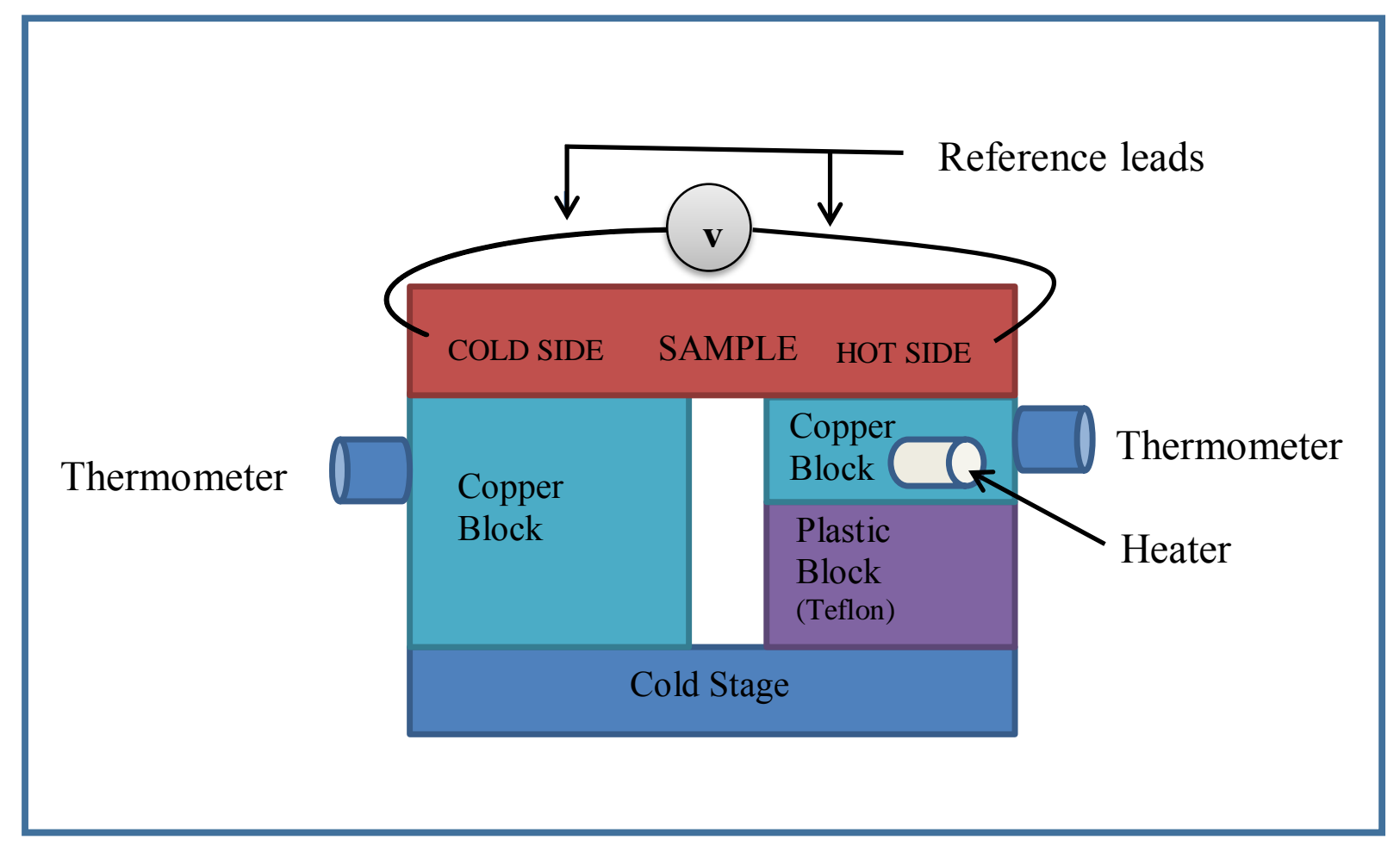

Figure 4.5: Block diagram of the thermopower rig. 


\subsubsection{Thermopower measurement}

The thermopower or Seebeck coefficient $(S)$ is the direct conversion of temperature difference to electric voltage and vice-versa. $S$ can be written as

$$
S=\frac{\Delta V}{\Delta T}
$$

In order to compute $S$, we need to calculate $\Delta V$ and $\Delta T$ from the thermopower experiment. The absolute thermopower of a material cannot be measured directly as a closed circuit is required to obtain the potential difference of the sample. This potential difference also includes the thermal voltage in the reference leads. In order to measure the thermal voltage of the sample alone, it is necessary to determine the relationship between the $\Delta V$ measured, and the thermal voltages of the sample and reference leads. Consider the thermopower circuit in Figure 4.6 to calculate $\Delta V[154,171]$.

$$
\begin{aligned}
\Delta V & =V_{a}-V_{b} \\
& =\left(V_{a}-V_{d}\right)+\left(V_{d}-V_{c}\right)+\left(V_{c}-V_{b}\right) \\
& =\int_{d}^{a} d V+\int_{c}^{d} d V+\int_{b}^{c} d V \\
& =-\int_{d}^{a} S_{\text {leads }} d T-\int_{c}^{d} S_{\text {sample }} d T-\int_{b}^{c} S_{\text {leads }} d T \\
& =-\int_{T 1}^{T 0} S_{1} d T-\int_{T 2}^{T 1} S \mathrm{~s} d T-\int_{T 0}^{T 2} S_{1} d T \\
& =-\int_{T 1}^{T 2} S_{1} d T-\int_{T 2}^{T 1} S \mathrm{~s} d T \\
& =\int_{T 1}^{T 2}\left(S_{S}-S_{1}\right) d T \\
& =\int_{T 1}^{T 2}\left(S_{\text {relative }}\right) d T
\end{aligned}
$$

So for a small temperature difference, $\Delta T=T_{2}-T_{1}$

$$
S_{r}=S_{s}-S_{1}=\frac{\Delta V}{\Delta T}
$$


Now it is clear that $\Delta V$ is the difference between the thermal voltages in the sample and the reference leads. In order to obtain the absolute thermopower of the sample, the thermopower of the reference leads must be known. In our case, we have used manganin wire as reference leads. The absolute thermopower value of manganin has been taken from D. Rathnayaka's article [172]. Manganin's thermopower has been shown in the Appendix A (Figure A.1).

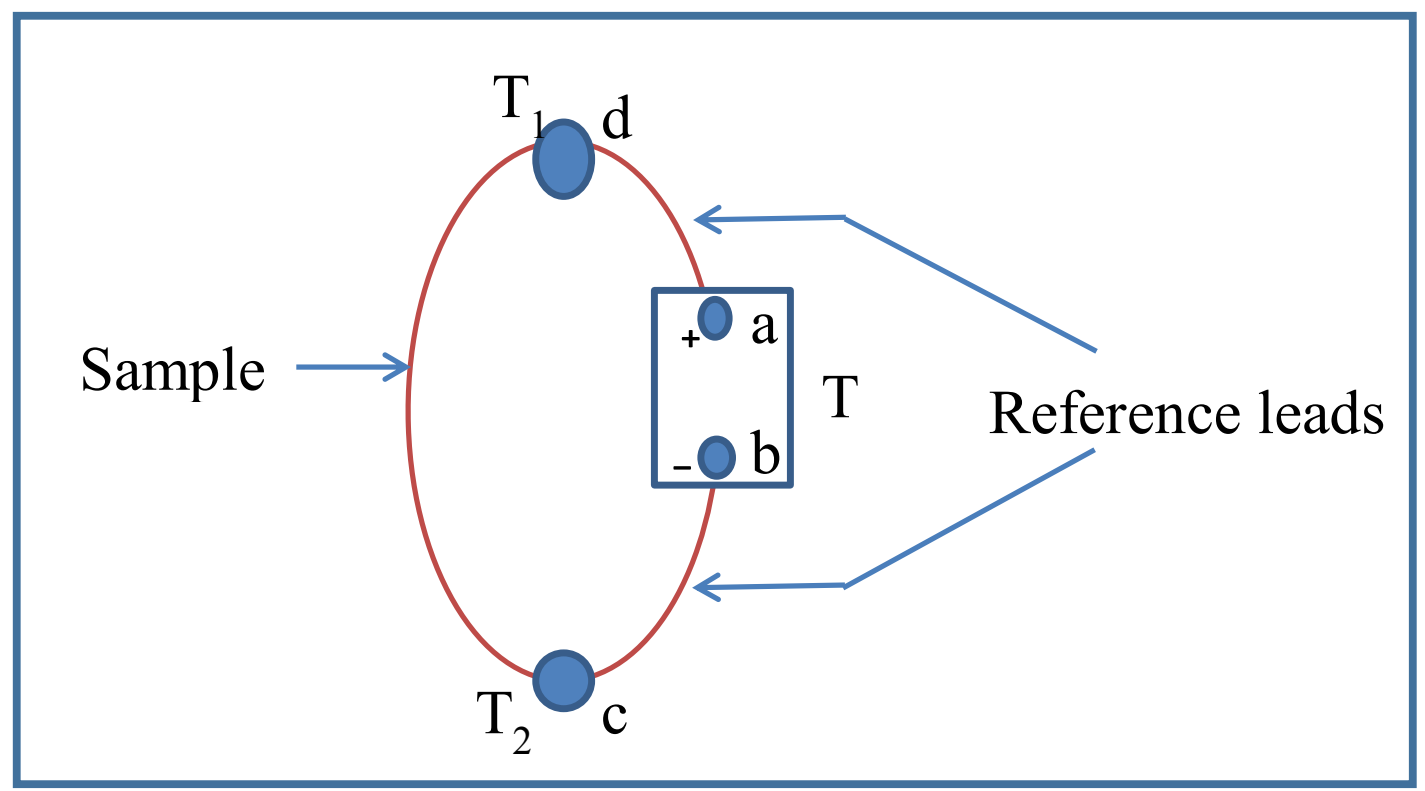

Figure 4.6: Thermopower circuit.

\subsubsection{Methods for measuring thermopower}

There are two different methods to measure the thermopower of a sample: the integral method and the differential method $[154,171]$. Both are based on

$$
\Delta \mathrm{V}=\int_{T 1}^{T 2}\left(S_{\text {relative }}\right) d T
$$

I. The Integral Method: In this method one end of the sample is kept at a known constant temperature $T_{1}$ while the temperature at the other end is increased to $T_{2}\left(T_{2}>T_{1}\right)$. The thermal voltage $V\left(T_{2}\right)$ across the sample and the temperature of the hot end are recorded over the defined range. The relative thermopower of the sample at the higher temperature $\left(T_{2}\right)$ is obtained by subtracting this data. 
II. The Differential Method: In this method a small temperature difference, $\Delta T=T_{2}-T_{1}$, is maintained across the sample, and the corresponding thermal voltage $\Delta V$ is measured. Generally the temperature of one side of the sample (hot end) is increased at a relatively faster rate than the other side (cold end) to create a temperature difference.

The relative thermopower of the sample at the average temperature $\frac{1}{2}\left(T_{1}+T_{2}\right)$ is then calculated using $S=\frac{\Delta V}{\Delta T}$.

We used the differential method in our measurements since it has some advantages over the integral method. Firstly, the thermal voltage $\Delta V$ can be measured directly using the differential method, whereas in the integral method one measures $\Delta V$ as the difference of two relatively large voltages. Secondly, the time interval in the differential method is small during the measurement, so the drift of the voltage has a relatively small effect on the accuracy of $\Delta V$. Any zero drifts in the thermal voltage that build up while the sample comes to an equilibrium temperature can be nulled off before heating the hot end of the sample to apply the temperature gradient.

\subsubsection{Thermal voltage measurement technique inside the cryostat}

The experiment was performed inside the cryostat under a vacuum. Two DT - 470 - CU silicon diode thermometers were attached to two copper blocks to monitor the temperature difference between the two ends of the sample as shown in Figure 4.7. These thermometers can measure temperatures between $1.4 \mathrm{~K}$ and $375 \mathrm{~K}$ with high accuracy. We bought these thermometers from Lake Shore Cryotronics. The instructions, provided from Lake Shore Cryotronics, were followed to mount these thermometers to the copper blocks. To ensure good thermal contact between the sensor (thermometer) and $\mathrm{Cu}$ mounting surface, we used a thin layer of Apiezon $\mathrm{N}$ grease between the mounting and $\mathrm{Cu}$ surface. It is important to note that an overabundance of grease can increase the thickness of the thermal barrier, which makes the temperature uncertain. To avoid this problem, we kept the thickness of grease to $0.05 \mathrm{~mm}$ or less [170]. The two thermometers were connected in series and required 10 microamperes current to operate. A Lakeshore 101 constant $10 \mu \mathrm{A}$ current source was used for these thermometers. 


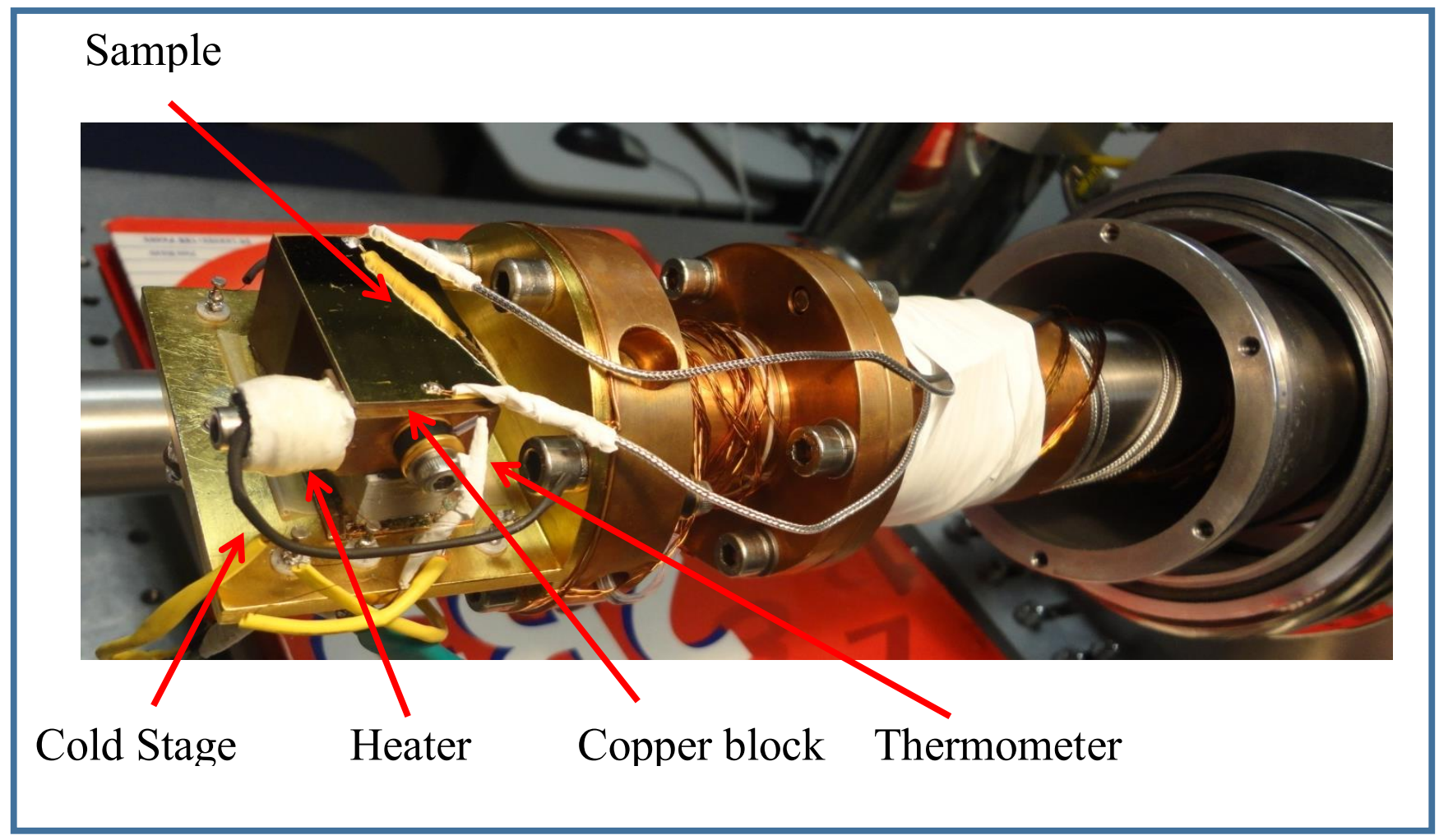

Figure 4.7: Thermopower set-up inside the cryostat in the low temperature lab.

Both thermometers recorded the voltage reading and each voltage reading corresponded to a specific temperature. The voltage and temperature relationship we obtained from the standard Curve 10 data (see Appendix B.1), supplied with the thermometers. For a small temperature range, the voltage vs temperature curve was treated as a straight line (Appendix Figure B.1). The sensitivity, $d V / d T$ was the same order for both thermometers and was considered as a constant value. For each set of readings, the average temperatures of the hot and cold ends $\left[T_{C}\right.$ (ave) and $T_{h}$ (ave)] were evaluated by taking the average voltage reading of each thermometer through LabView programming.

One heater was attached to a small copper block to maintain the temperature gradient. It was tightly bound by Teflon tape, otherwise the inside constantan wires would fall off. The Yokogawa 7651 programmable DC current source was used to maintain the heater current. We used a piece of a plastic Teflon block as a good insulator between the cold stage and the small copper block. 
The sample was placed on the top of the two copper blocks. We needed glue to stick the sample on the copper block. The glue had to be a good thermal conductor, otherwise the sample and copper block would be at different temperatures. GE 7031 varnish was used for the thermal contact. The voltage of the thermometers of the two sides of the sample was recorded by a Tektronix DMM 40406 digit precision multimeter and then this recorded voltage compared with the standard Curve data through the LabView programming in order to show which voltage corresponds to what temperature (Appendix Figure B.1). The thermal voltages were measured using a Keithley 181 nanovoltmeter, using the thermopower reference leads. The two reference leads were made the same length, glued together and passed by a small bobbin, so that the inhomogeneities were uniformly distributed and the spurious thermal voltages would be cancelled out. Indium was used to make contacts on the top of the sample to connect the reference leads, as it is reliable at low temperatures. It was also necessary to maintain all the leads and connections outside the cryostat at the same temperature to minimise the spurious thermal voltages. This was done by keeping them well shielded and thermally anchored.

After mounting the sample on the top of the copper block, we checked all connections and performed the experiment at room temperature. Initially, the temperature of the hot side and the cold side was approximately equal and the initial values of $V_{1}, V_{2}$ (thermometer voltages) and the corresponding thermal voltage $V$ were recorded. Then the temperature of the sample's hot side was increased in small steps and at each step the values of $V_{1}, V_{2}$ and $V$ were taken when the hot side's temperature was stabilized. The temperature difference $(\Delta T)$ had to be small enough so that the $\Delta V-\Delta T$ plot remained linear over one set of readings.

After successfully measuring the test experiment at room temperature, we closed the cryostat and ran the backing pump until the pressure inside the cryostat reached around $1 \times 10^{-2}$ mbar. Once it achieved the desired pressure we turned on the turbo pump to get a lower pressure of around $1 \times 10^{-8}$ mbar and performed the test experiment again under the vacuum. After verifying the RT result, we allowed the sample to cool down to $4 \mathrm{~K}$ by running the compressor overnight. During this Ph.D., the LabView code has been written for this measurement that effectively and automatically operated the system and control measurement processes. 


\subsubsection{System calibration, substrate contribution and uncertainty measurement}

There are a few things which we need to consider carefully for the thermopower measurement. Firstly, one would argue about the system calibration. The calibration of the thermopower setup is essentially impossible because there are no known materials for which the thermopower is sufficiently independent of small defect concentration. It depends on how the film was grown and various other parameters [151 - 154]. As a consequence, what one needs to do is make sure that the measurement is done correctly. It is necessary to make sure we have measured the temperature difference and voltage difference correctly between two ends of the sample and use reference leads of which the thermopower value is known.

Choosing the right substrate is very crucial for thermopower measurement as it can dominate the magnitude of the measured thermopower. In order to ensure both sides of the films are at the same temperature we have to have good thermal conducting layers between the $\mathrm{Cu}$ blocks and sample as stated earlier. In addition to that, we need to maintain the poor thermal conduction along the substrate. Otherwise, heat will flow along the substrate and affect the temperature difference between the two ends of the film.

Therefore, substrates with low thermal conductivity are the ideal candidates for depositing REN films for conducting this measurement. Otherwise, wrong thermopower result will be achieved. To perform this measurement, we have to be extremely cautious about the heat path and in the direction of the temperature gradient. For instance, silicon and sapphire substrates have shown to have higher thermal conductivity and found to affect the measurements of films on these substrates. Thus they significantly dominate the thermopower measurement of the REN films. In contrast, glass and YSZ substrates are a better choice to perform this experiment because they exhibit lower thermal conductivity.

All experimental measurements contain some sort of uncertainty. The uncertainty of this measurement arises mainly from the calibration of the thermometer, the attached heater, the length of reference leads, temperature variation of the system and sample mounting process. It is difficult to predict each of this contribution accurately. The best we could say the uncertainty of our experiment is $\pm 1 \mu \mathrm{V} / \mathrm{K}$. 


\section{Chapter 5}

\section{Magnetotransport properties of high carrier concentration epitaxial gadolinium nitride film (GdN_HC)}

\subsection{Concept of nitrogen vacancies}

In the next three chapters, we will discuss the results from three different GdN films grown using different growth conditions to see the correlation between the nitrogen vacancies $\left(V_{N}\right)$ concentration and film properties. Thus, before we start showing our results it is useful to consider some basic concepts associated with the $V_{N}$ states.

Figure 5.1(a) shows a simple schematic illustration of the electronic band structure of GdN. The valence band of $\mathrm{GdN}$ is predominantly comprised of $\mathrm{N} 2 p$ orbitals and the conduction band is formulated from Gd $5 d$ orbitals as stated earlier (Chapter 1). When the temperature is reduced and the ferromagnetic state is achieved, the bands spin-split due to the exchange interaction that originates from the half-filled Gd $4 f$ band.

The Fermi level of an experimentally prepared GdN film is defined by the presence of impurity states that are generally associated with nitrogen vacancies $\left(V_{N}\right)$ that give rise to the $n$-type doping of the films. According to a theoretical calculation by Punya et al. [173] each nitrogen vacancy can provide up to three electrons. Among them two electrons of opposite spin are bound strongly in impurity states located in the GdN band gap while the third electron works as a free carrier in the conduction band. The authors also noted that at more modest $V_{N}$ concentrations the third electron is more likely to occupy a shallow donor level below the conduction band, from where it can be thermally excited to the extended conduction band states 
[173]. It is of significant interest to test whether the validity of this description for films with different nitrogen vacancy concentrations.

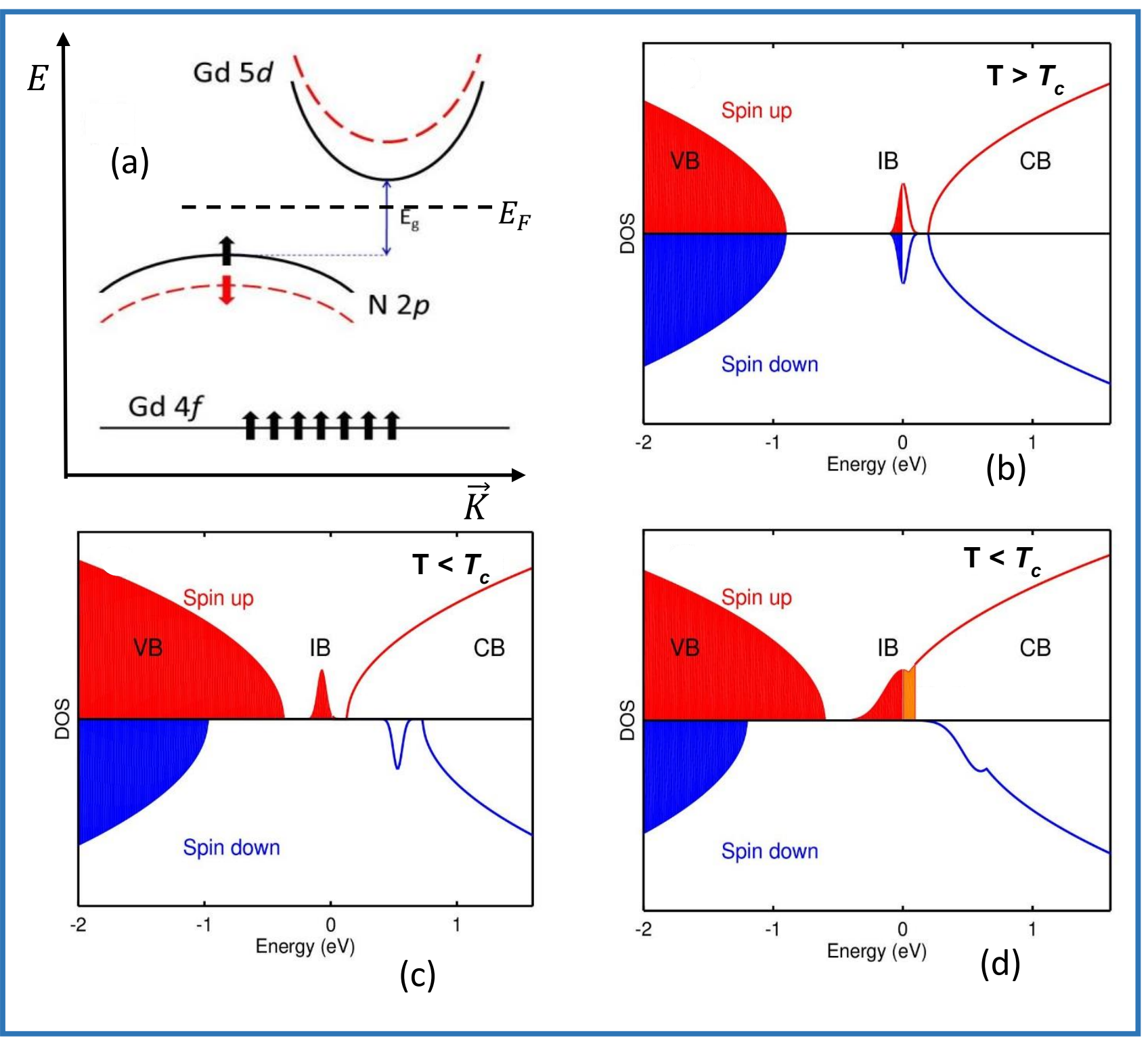

Figure 5.1: (a) Schematic band diagram of GdN. (b) Schematic density of states, where the Fermi level is at $\mathrm{E}=0$ when $T>T_{c}$, no spin spliting. (c) Fermi level is at $\mathrm{E}=0$ when $T<T_{c}$, less nitrogen vacancy concentration. (d) Fermi level is at $\mathrm{E}=0$ when $T<T_{c}$, high nitrogen vacancy concentration. Spin-up $($ red color $)=$ majority-spin, spin-down $($ blue color $)=$ minority-spin .

We begin by considering how nitrogen vacancies might affect the density of states using a model based on conventional semiconductor physics involving only impurity states in the band gap. This model has formed the basis of a description involving magnetic polarons of the magnetism in $\mathrm{GdN}$ [97]. We will later show that the results of the present thesis require a 
revision of this picture to include impurity states lying above the conduction band minimum. Figure 5.1 (b,c,d) illustrate a schematic spin-resolved density of states for GdN including a contribution from a vacancy induced impurity level. The red and blue colours show the spinup and spin-down states, respectively. The shaded areas represent occupied states below the Fermi level.

For low $V_{N}$ concentrations it is assumed that a narrow impurity band forms in the gap just below the conduction band. Figure 5.1 (b) represents the paramagnetic state for the low impurity concentration case when the temperature is much higher than the transition temperature so there is no splitting between the up and down spins. The impurity band is assumed to be partly occupied for each spin. Below the transition temperature, exchange splitting plays a significant role and narrows the band gap for majority-spins. Thus, the impurity band extends closer to the minimum of the conduction band. The impurity band states also experience a strong exchange interaction and resulting spin splitting comparable to that in the conduction band. This situation is illustrated in 5.1 (c), where only the impurity band of majority-spin is occupied, and the lower conduction band is also purely majority-spin. For a high level of nitrogen vacancies $\left(\sim 10^{20} \mathrm{~cm}^{-3}\right)$ the impurity levels will begin to overlap strongly, and the impurity band will broaden and merge with the conduction band minimum as shown in Figure 5.1 (d). States in the bottom of the conduction band may be localised (shaded in orange here) leading to a mobility edge.

This chapter presents the result of magnetic and electron transport phenomena on a high carrier concentration epitaxial gadolinium nitride $(\mathrm{GdN})$ film which is grown by molecular beam epitaxy (MBE) using ammonia $\left(\mathrm{NH}_{3}\right)$ as the nitrogen precursor. A $120 \pm 10 \mathrm{~nm}$ thick GdN film was deposited at $750{ }^{\circ} \mathrm{C}$ on a $50 \mathrm{~nm}$ thick AlN template coated respectively on a silicon substrate in the MBE laboratory at Valbonne, France.

There are two main sections in this chapter to report the magnetic and transport results of the epitaxial GdN_HC (HC stands for high carrier concentration) film, followed by a summary and conclusion. The main emphasis has been put on the electron transport results where some magnetic results are required to elaborate the overall discussion. As transport results are the heart of this chapter, thus we have comprised this lengthy section with five different subsections where first and second subsections discuss the temperature dependent resistivity and the magnetoresistance results of this film, respectively. The third and fourth subsections report the Hall effect result and the interpretation of this result. A fifth subsection is employed to describe the first-ever thermopower result along with the interpretation of the result. 


\subsection{Magnetic results of the GdN_HC film}

Magnetisation measurements of the epitaxial GdN film were carried out from $2 \mathrm{~K}$ to $300 \mathrm{~K}$ using a Quantum Design SQUID magnetometer with the field oriented in-plane. The experiment was performed in the presence of a small applied magnetic field of $250 \mathrm{Oe}$, which is higher than the typical value of the coercive field of the film. A plot of the magnetisation as a function of temperature for the epitaxial GdN_HC is shown in Figure 5.2.

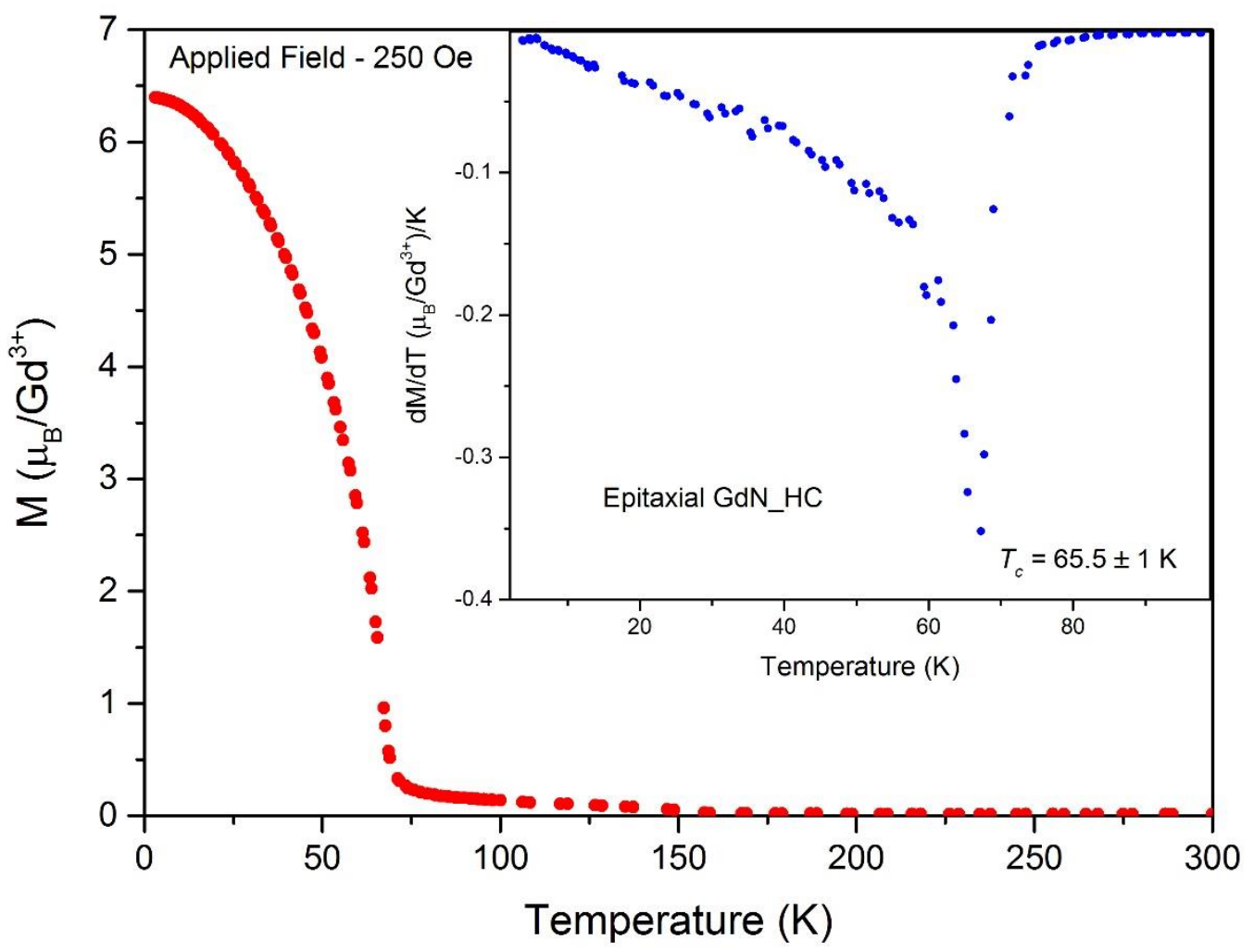

Figure 5.2: Magnetisation curve and first derivative (inset) of the epitaxial GdN film_HC as a function of temperature.

The result shows classical ferromagnetic behaviour. At low temperature, the magnetisation shows an upturn below $65.5 \pm 1 \mathrm{~K}$, indicating the ferromagnetic Curie temperature is in this region. To further verify the transition temperature, the first-order derivative of the magnetisation with respect to the temperature was calculated as shown in the inset of Figure 5.2. A minimum is seen at $65.5 \pm 1 \mathrm{~K}$ which is taken as the ferromagnetic transition temperature of this film. Near this temperature strong magnetic fluctuations are prominent which 
significantly affect the transport properties, as we will see in our magnetoresitance and mobility results discussed in Sections 5.3.2 and 5.3.3, respectively.

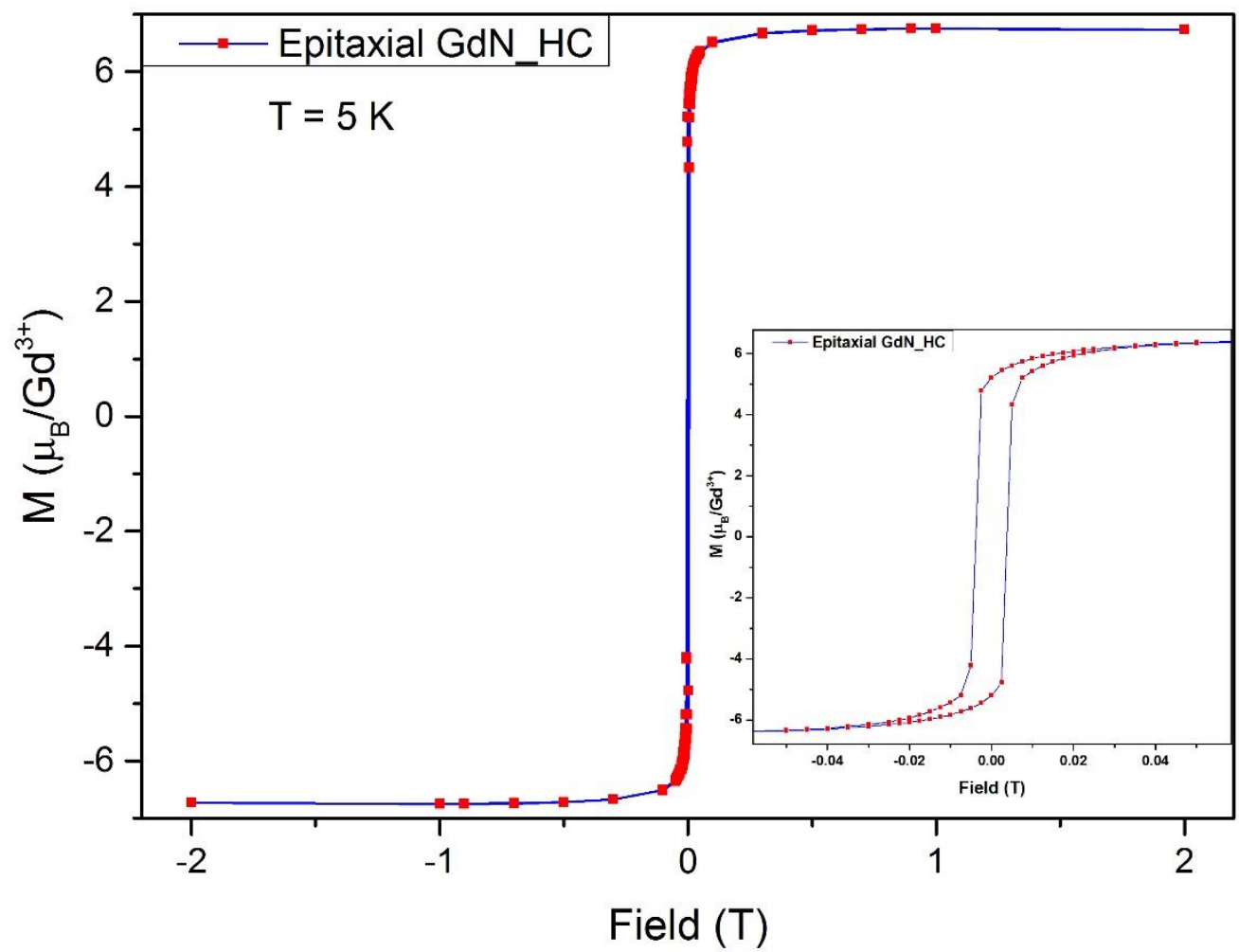

Figure 5.3: Magnetisation data of the epitaxial GdN_HC film as a function of field at $5 \mathrm{~K}$. Inset: the hysteresis loop of the epitaxial GdN_HC film at $5 \mathrm{~K}$.

Figure 5.3 shows the field dependent magnetisation measurements performed at $5 \mathrm{~K}$. The external magnetic field was applied along the film's surface. The magnetisation of the film rises with weaker applied fields (below 2T) and then saturates in the presence of magnetic fields above $\pm 2 \mathrm{~T}$.

It is clear from Figure 5.3 that the magnetisation becomes saturated very quickly with the applied field directed in-plane at low temperature. The saturation magnetisation at $5 \mathrm{~K}$ was found to be $6.5 \pm 0.5 \mu_{\mathrm{B}} / \mathrm{Gd}^{3+}$ for this epitaxial film which is close to the expected value of 7 $\mu_{\mathrm{B}} / \mathrm{Gd}^{3+}$. In order to determine the magnetisation in Bohr magnetons per gadolinium ion $\left(\mu_{\mathrm{B}} / \mathrm{Gd}^{3+}\right)$, the volume of the film must be known, which dominates the uncertainty in this experiment. As we cannot measure the thickness of the film in-situ, we have used the thickness obtained from SEM measurements. The area of the film was calculated manually using a ruler 
and optical microscope, where one would further expect a smaller contribution of uncertainty from this measurement.

Hysteresis curves provide further evidence about the ferromagnetic state of the GdN_HC film as shown in Figure 5.3 inset. The film shows a very narrow hysteresis and a small coercive field. A coercive field of $40 \mathrm{Oe}$ and remanence of $5.5 \mu \mathrm{B} / \mathrm{Gd}^{3+}$ were extracted from the hysteresis curve at $5 \mathrm{~K}$ for this epitaxial $\mathrm{GdN}$ film. The small coercive fields indicate that the film requires only 40 Oe field to demagnetise which is characteristic of soft magnetic nature of the film. 


\subsection{Transport results of the GdN_HC film}

\subsubsection{Resistivity result}

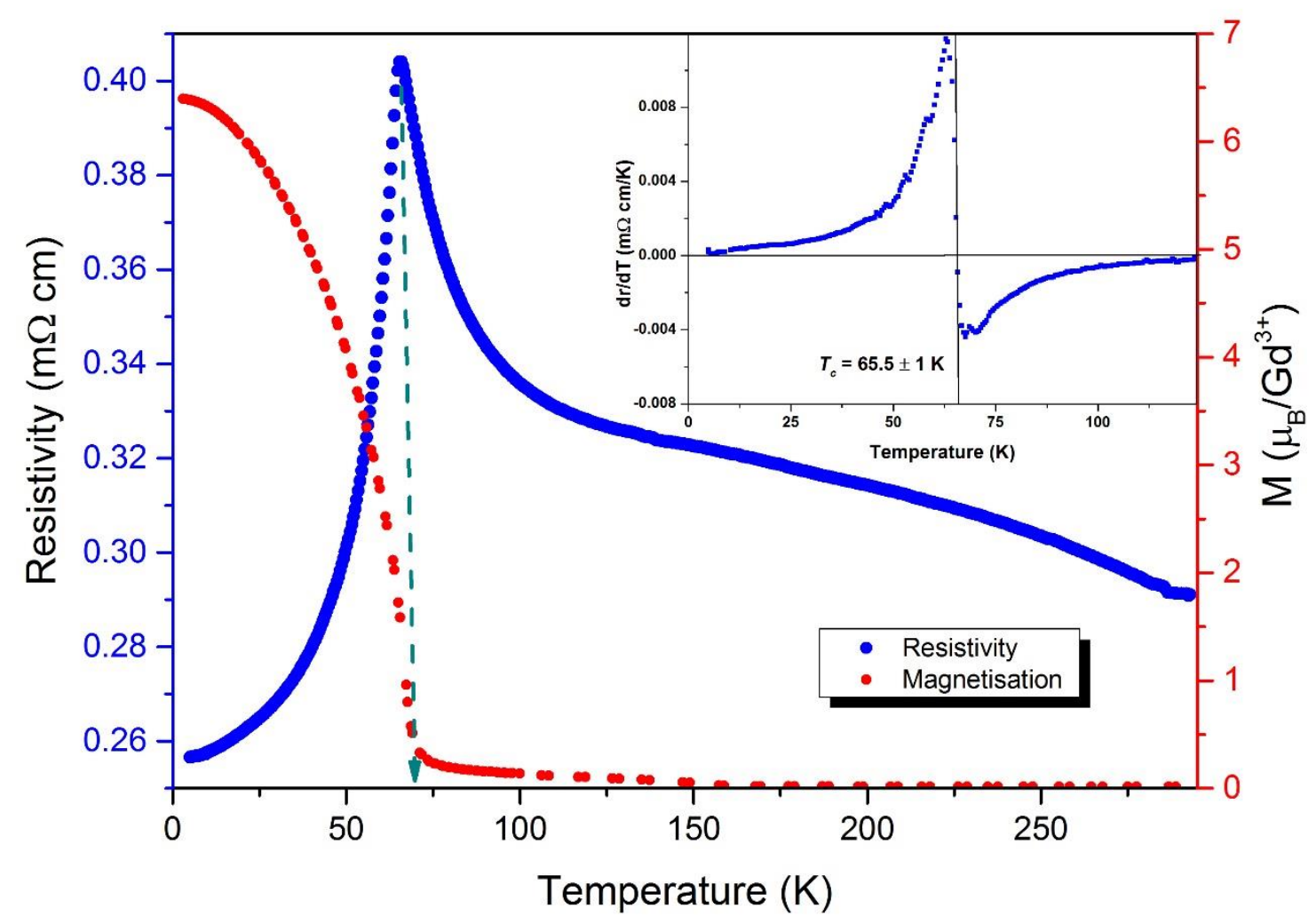

Figure 5.4: Temperature dependent resistivity and magnetisation at 250 Oe of the epitaxial GdN_HC film. Inset: Temperature derivative of the resistivity of the same GdN film.

Figure 5.4 shows the resistivity and magnetisation results as a function of temperature for the epitaxial GdN_HC film. The inset figure shows the temperature derivative of the resistivity that displays the inflection point at $65.5 \pm 1 \mathrm{~K}$ which correlates nicely with the Curie temperature obtained from the magnetic measurement.

The resistance of this film is found to be low. As the temperature decreases from ambient temperature, the resistivity of the sample increases and shows a very sharp cusp at around 65.5 $\pm 1 \mathrm{~K}$. In the paramagnetic region the negative temperature coefficient of resistance (TCR) is too weak to ascribe to carrier activation, in agreement with Hall measurements reported later 
in this chapter. In the ferromagnetic state below the transition temperature the resistivity decreases sharply with the lowering of temperature, showing a strong positive temperature dependence of resistance (TCR). It will be confirmed later that the carrier concentration does not show a corresponding rapid change near $T_{c}$, so the resistivity peak must be associated with a peak in the scattering rate. Since the peak occurs where magnetic fluctuations are strongest it is natural to assume it is caused by magnetic disorder scattering. Many years ago the resistivity anomaly at the transition temperature of a metallic ferromagnet had been studied by de Gennes and Friedel [174]. They have predicted that the cusp like peak in the resistivity results from long-range fluctuations of the magnetisation near the transition temperature. Later Fisher et al. suggested that it is actually the short-range fluctuations which make the dominant contribution to the temperature dependent part of the resistivity [175]. The same authors assumed in their calculation of scattering of conduction electrons that the spin fluctuations were relatively slow, i.e. the amount of energy that an electron exchanges in a spin-flip scattering is negligible. The shape of the resistivity near $T_{c}$ depends on the relative density of charge carriers and magnetic scattering centres [53]. The magnetic scattering centres are basically every magnetic ion in the material. When the density of charge carriers is similar to the density of scattering centres there is no sharp resistivity peak. It can only occur when the carrier concentration is much smaller than the scattering centre density.

It is to be noted that this type of a very sharp cusp is not seen in other metallic ferromagnetic metals (e.g., Ni, Fe) or dilute magnetic semiconductors (e.g., GaMnAs) [97, 176] where the density of magnetic sites is very similar to the density of conduction electrons. For GdN every Gd ion acts as a magnetic scattering centre ( 7 electrons available in the $f$ orbital of Gd) whereas conduction electrons density is similar to the nitrogen vacancy concentration which is much less than the $\mathrm{Gd}$ concentration. $\mathrm{GdN}$ is thus in a new regime with low carrier concentration but high magnetic ion density. Note that the low resistivity value and the sharp resistivity cusp imply that the film is behaving essentially as a metal with conductivity defined by fully delocalised electrons in the conduction band. 


\subsubsection{Magnetoresistance result}

The magnetic results show that the strong magnetic disorder scattering maximises at the Curie temperature so that the resistivity falls strongly in the ferromagnetic state below $\sim 65 \mathrm{~K}$. This result clearly indicates that magnetic scattering can significantly influence the conductivity of the film, even in the presence of an external magnetic field. Thus, we have performed magnetoresistance (MR) measurements on the same epitaxial film at various temperatures as a function of field applied both parallel and perpendicular to the film surface.

It is to be noted that we were mainly interested to collect the MR data especially in the ferromagnetic region as magnetic fluctuation plays a very significant role to dominate the resistance in this region. Thus the sequence file has been written in a way to collect the maximum set of data mainly between $80 \mathrm{~K}$ and $3 \mathrm{~K}$ temperature regions whereas $180 \mathrm{~K}$ was set as the starting temperature. 
The influence of strong magnetic scattering is clearly prominent in the magnetoresistance. Turning the field on reduces the magnetic disorder of the system which leads to negative MR value as seen our results.

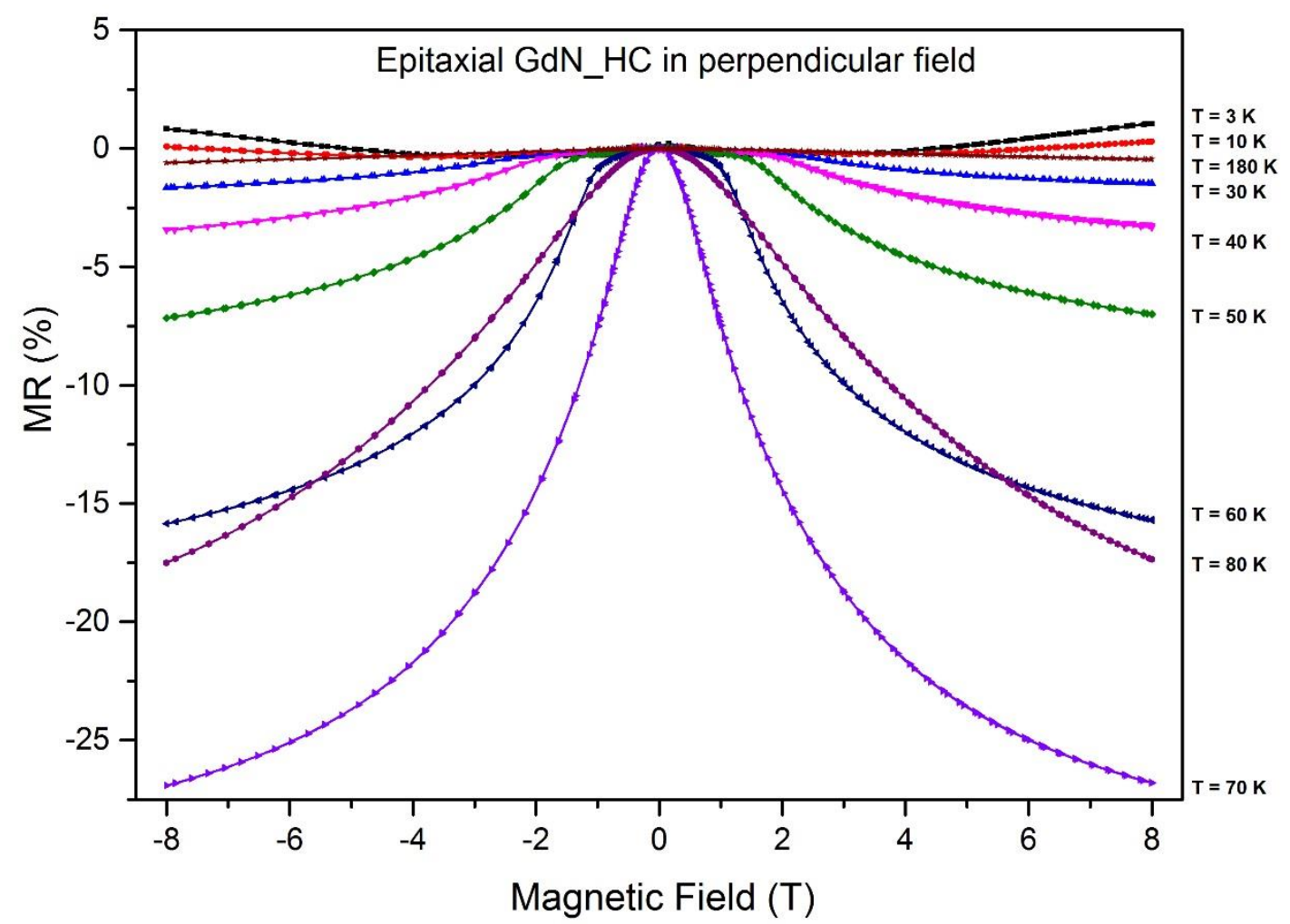

Figure 5.5: Magnetoresistance of the epitaxial GdN_HC film in applied fields perpendicular to the film surface. 


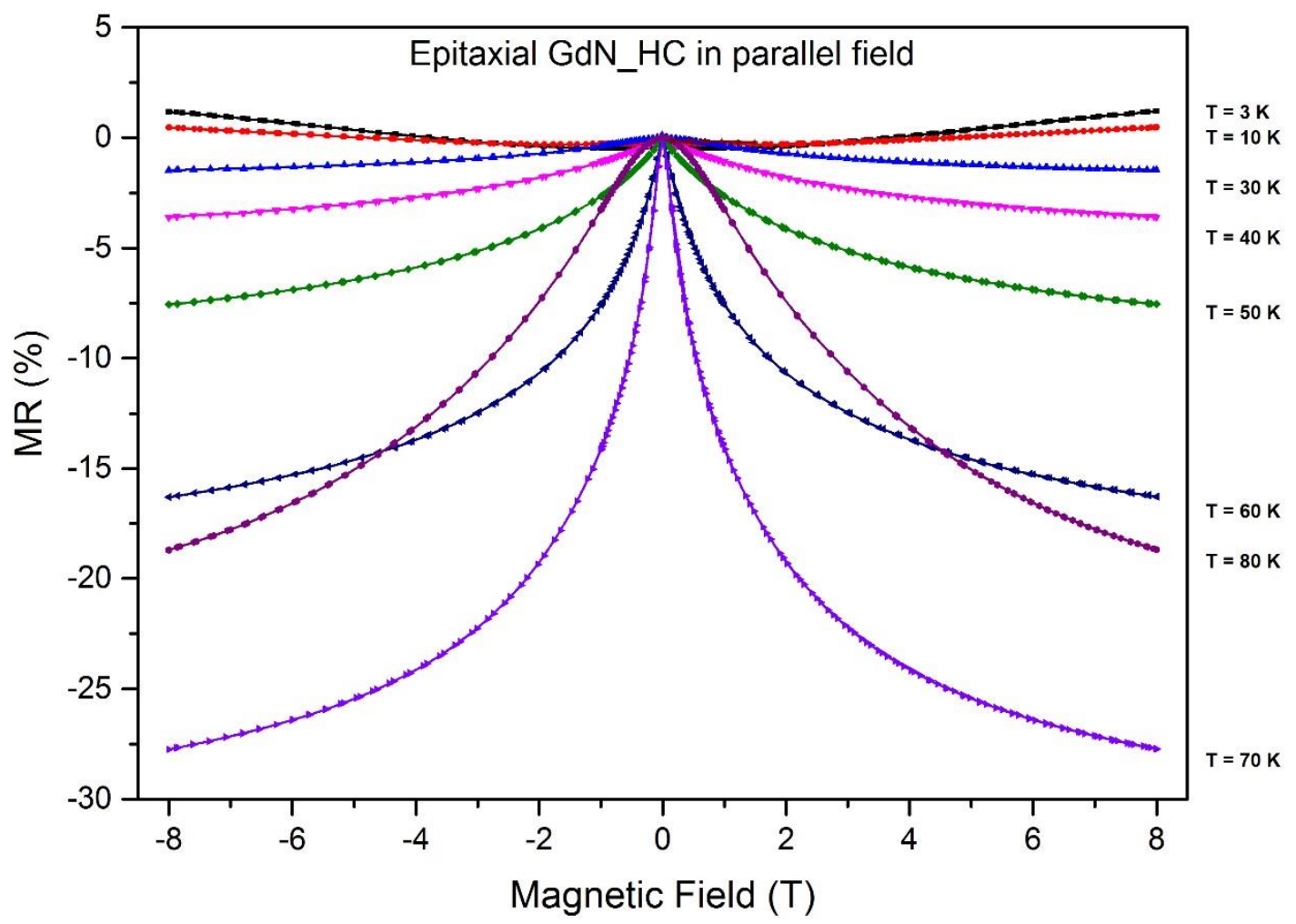

Figure 5.6: Magnetoresistance of the epitaxial GdN_HC film in applied fields parallel to the film surface.

Figures 5.5 and 5.6 show the magnetoresistance of epitaxial GdN_HC film measured at various temperatures from $3 \mathrm{~K}$ to $180 \mathrm{~K}$ for fields applied perpendicular and parallel to the film surface. The magnitude of the magnetoresistance is strongly temperature dependent. The maximum MR value $(-27 \%)$ is observed near the transition temperature in the presence of magnetic field of \pm $8 \mathrm{~T}$. All peaks below the transition temperature are much broader in the perpendicular field and a knee is clearly seen near the $2 \mathrm{~T}$ magnetic field. This is because of the shape anisotropy which requires a minimum $2.1 \mathrm{~T}(4 \pi \mathrm{M})$ magnetic field to achieve a saturation magnetisation for a GdN film. 
The change of MR as a function of temperature is shown in Figure 5.7. The Figure shows there is a little difference in the MR values obtained from the highest magnetic fields of $\pm 8 \mathrm{~T}$ when applied perpendicular and parallel to the sample surface. Interestingly, a significant difference can be seen for the two different field directions if we consider the MR value below $\pm 2 \mathrm{~T}$ due to the effect of shape anistropy. For example, at $70 \mathrm{~K}$, in the presence of $\pm 2 \mathrm{~T}$ field, the changes of MR values are $-15 \%$ and $-20 \%$, for the perpendicular and parallel applied field directions, respectively.

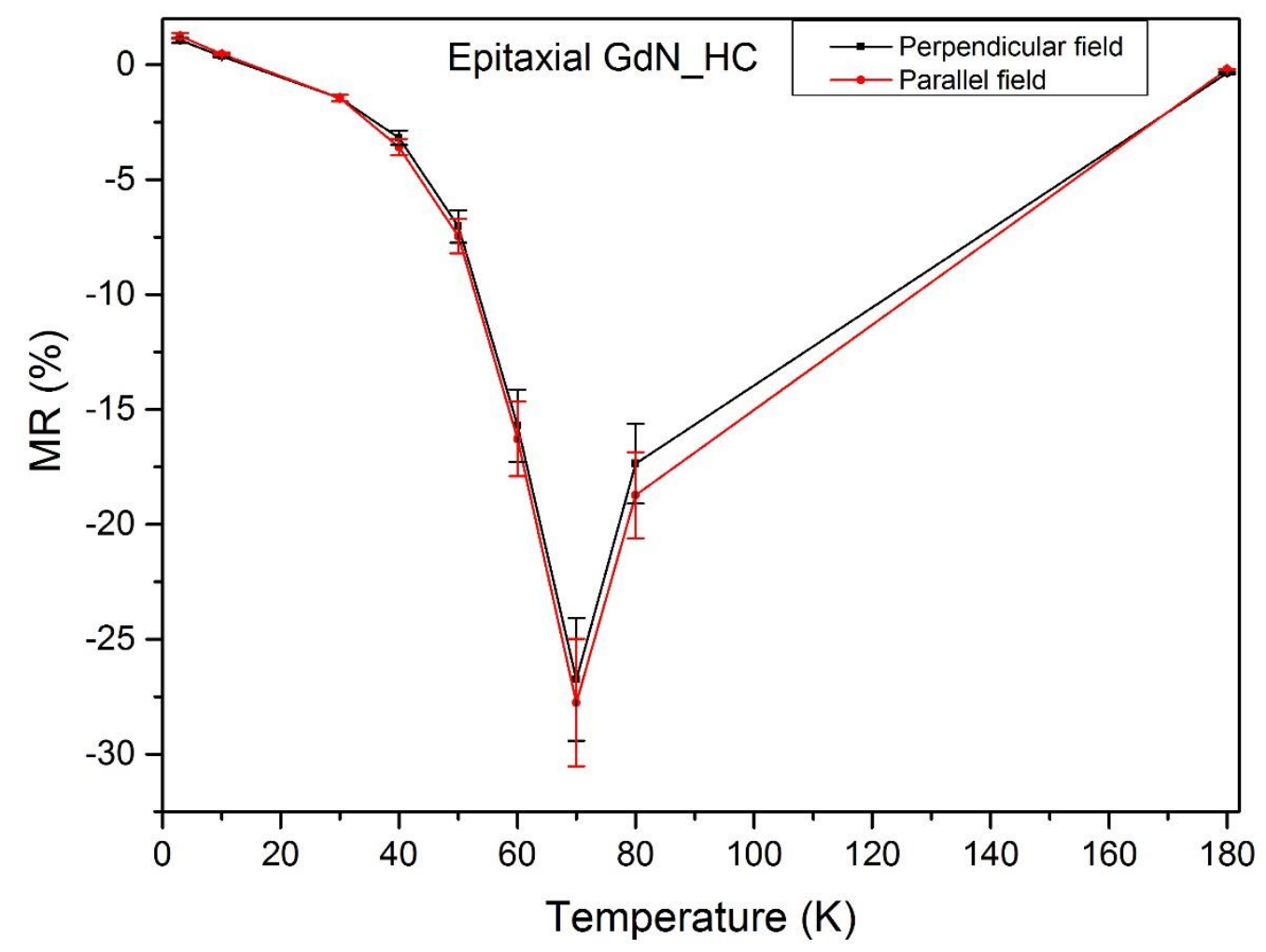

Figure 5.7: Temperature dependent magnetoresistance at different field orientation of the epitaxial GdN_HC film at $\pm 8 \mathrm{~T}$. 
An interesting feature is observed when the temperature is reduced below $15 \mathrm{~K}$. The sample shows a hysteresis in the magnetoresistance with an applied field parallel to the film surface at low temperature $T=10 \mathrm{~K}$ and $T=3 \mathrm{~K}$. At low temperature, the parallel field MR of the epitaxial film shows a sharp negative dip before turning positive [Figure 5.8 and 5.9], while in perpendicular field a hysteresis is seen in fields near $2 \mathrm{~T}$ and less than $0.5 \mathrm{~T}$. The first hysteresis loop at $2 \mathrm{~T}$ indicates a complicated interplay between the resistance and the domain structure in this field orientation whereas near $0.5 \mathrm{~T}$ it is related to the sample misalignment as discussed briefly in the following paragraphs.

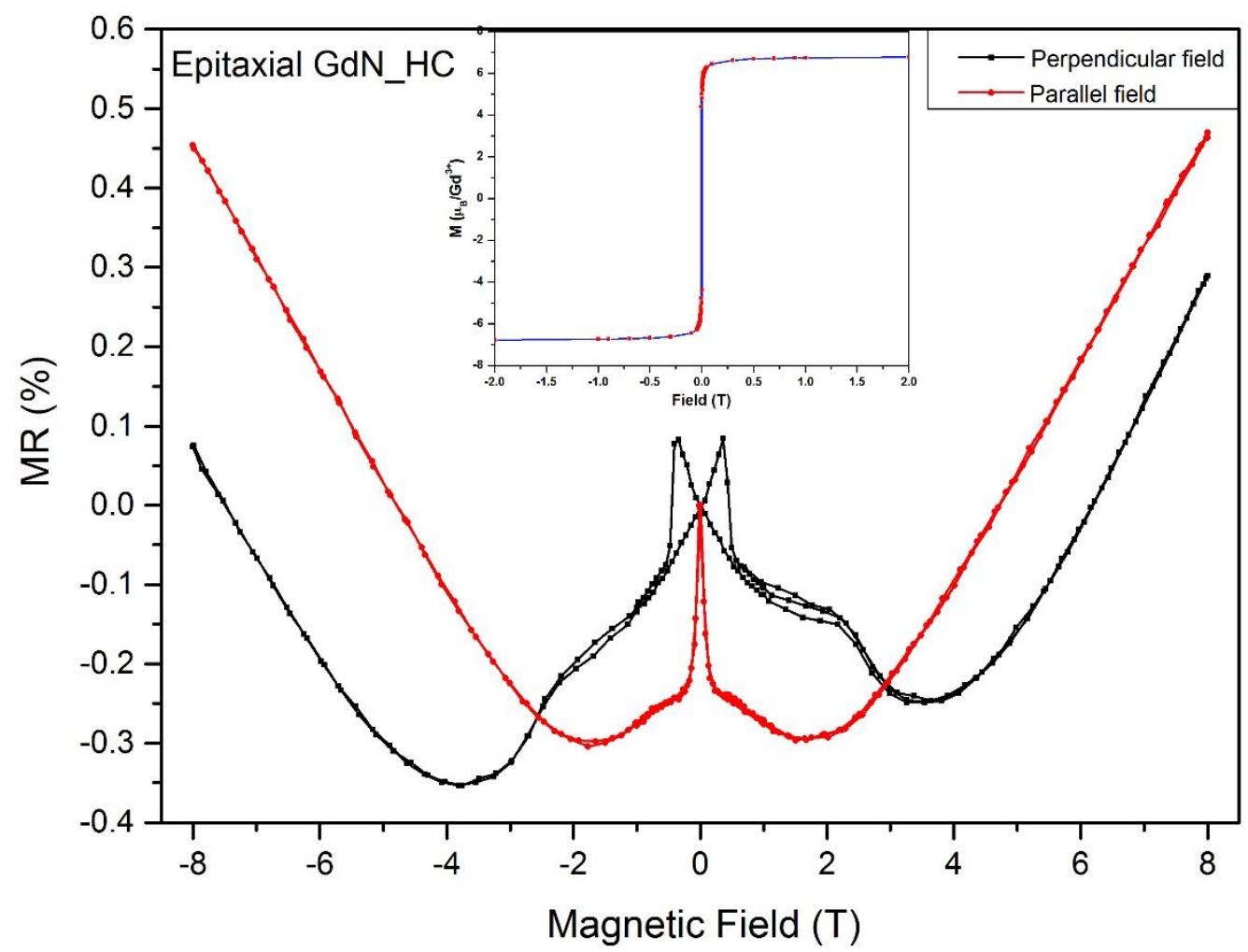

Figure 5.8: Magnetoresistance of the epitaxial GdN_HC film at $10 \mathrm{~K}$ measured with the applied magnetic field perpendicular (black line) and parallel (red line) to the film surface. Inset: magnetisation as a function of applied magnetic field parallel to the film surface at $10 \mathrm{~K}$. 


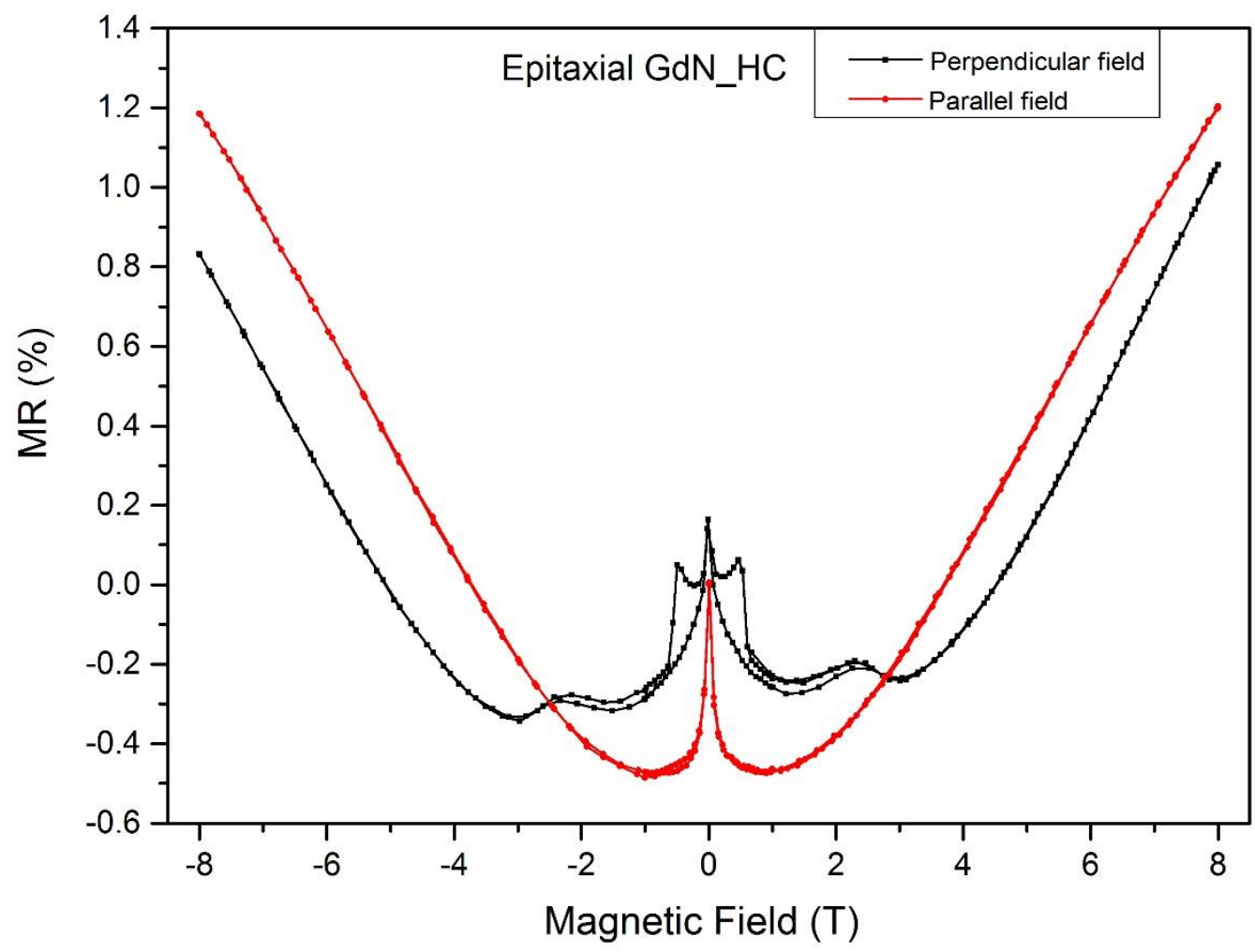

Figure 5.9: Magnetoresistance of the epitaxial GdN_HC film at $3 \mathrm{~K}$ measured with the applied magnetic field perpendicular (black line) and parallel (red line) to the film surface.

The peaks appearing around $\pm 0.5 \mathrm{~T}$ in Figure 5.9 are related to a small misalignment of the sample, so that the peak at $\sim 0.5 \mathrm{~T}$ signals the alignment of the magnetisation in-plane. If we consider a typical coercive field of epitaxial $\mathrm{GdN}$ of $100 \mathrm{Oe}$, then the misalignment of the sample could be estimated around $1^{\circ}$, which would then lead to peaks near $\pm 0.5 \mathrm{~T}$. It is then possible that the field is a few degrees off from normal to the film surface. This is likely to arise during the sample mounting process. 


\subsubsection{Hall effect and mobility results}

In order to explore the influence of the carrier concentration and mobility on the conductivity we measured the Hall effect as a function of magnetic field at different temperatures, shown for a few temperatures in Figure 5.10.

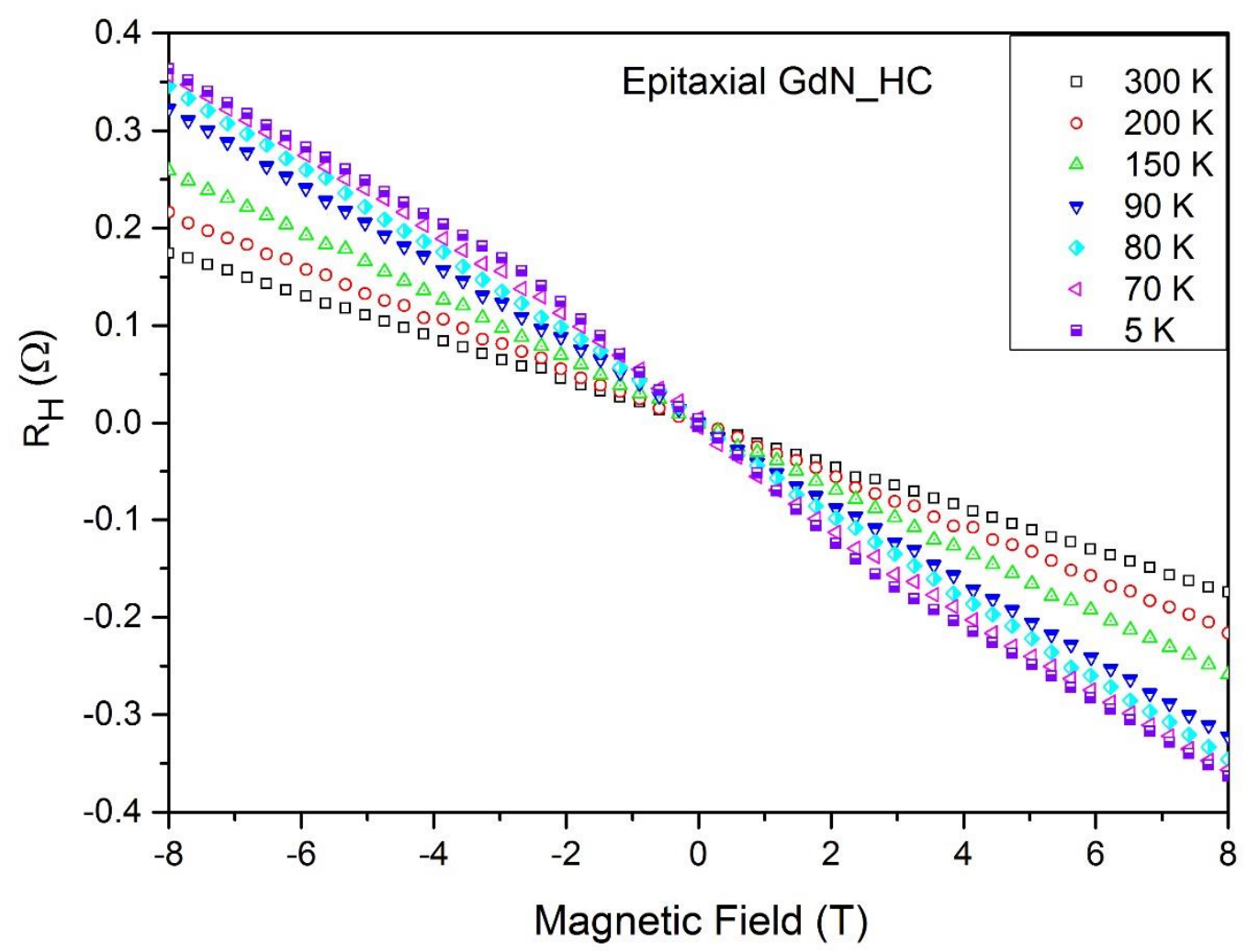

Figure 5.10: Hall resistance of the epitaxial GdN_HC film as a function of applied magnetic field at different temperatures.

The Hall effect was measured at different temperatures from $300 \mathrm{~K}$ down to $5 \mathrm{~K}$ and up to the highest applied magnetic field of $\pm 8 \mathrm{~T}$. The Hall data demonstrates a negative slope, indicating that the charge carriers are electrons, expected to originate from nitrogen vacancies.

The Hall resistance $\left(R_{H}\right)$ in the paramagnetic phase i.e. the temperatures at $300 \mathrm{~K}$ and $90 \mathrm{~K}$, shows a simple linear dependence, but below $80 \mathrm{~K}$ the field-linear component is augmented by the anomalous Hall effect which is proportional to the magnetisation and rises linearly with field until it saturates above $2.1 \mathrm{~T}$, i.e. for the out-of-plane configuration used for the Hall effect 
measurement, the shape anisotropy prevents full magnetic alignment until $\mathrm{B}=4 \pi \mathrm{M}(\sim 2.1 \mathrm{~T}$ for $\mathrm{GdN})$.

Within the usual single-band model, the ordinary Hall effect $(\mathrm{OHE})$ relates uniquely to the sign and concentration of the charge carriers. We thus use the OHE in the present case for obtaining the approximate concentration of the carriers from the high field slope to see how it influences the conductivity of the film at different temperatures. The OHE is extracted from the high-field slope, where the magnetisation is saturated and the AHE represents only a constant offset to the Hall resistance. We will show in the following chapter that one must in general be careful when using this procedure at tempertures just above $T_{c}$, where the magnetisation may not saturate even in $8 \mathrm{~T}$. For the present film the most interesting results come in the regions below and well above $T_{c}$. It is to be noted that, the uncertainty of the value of the carrier concentration is $\pm 10 \%$ which mainly arises from uncertainty in the thickness of the film and is shown by the error bar in the same figure. The resulting inverse of the slope (or carrier concentration) at different temperatures as a function of temperature is shown in Figure 5.11.

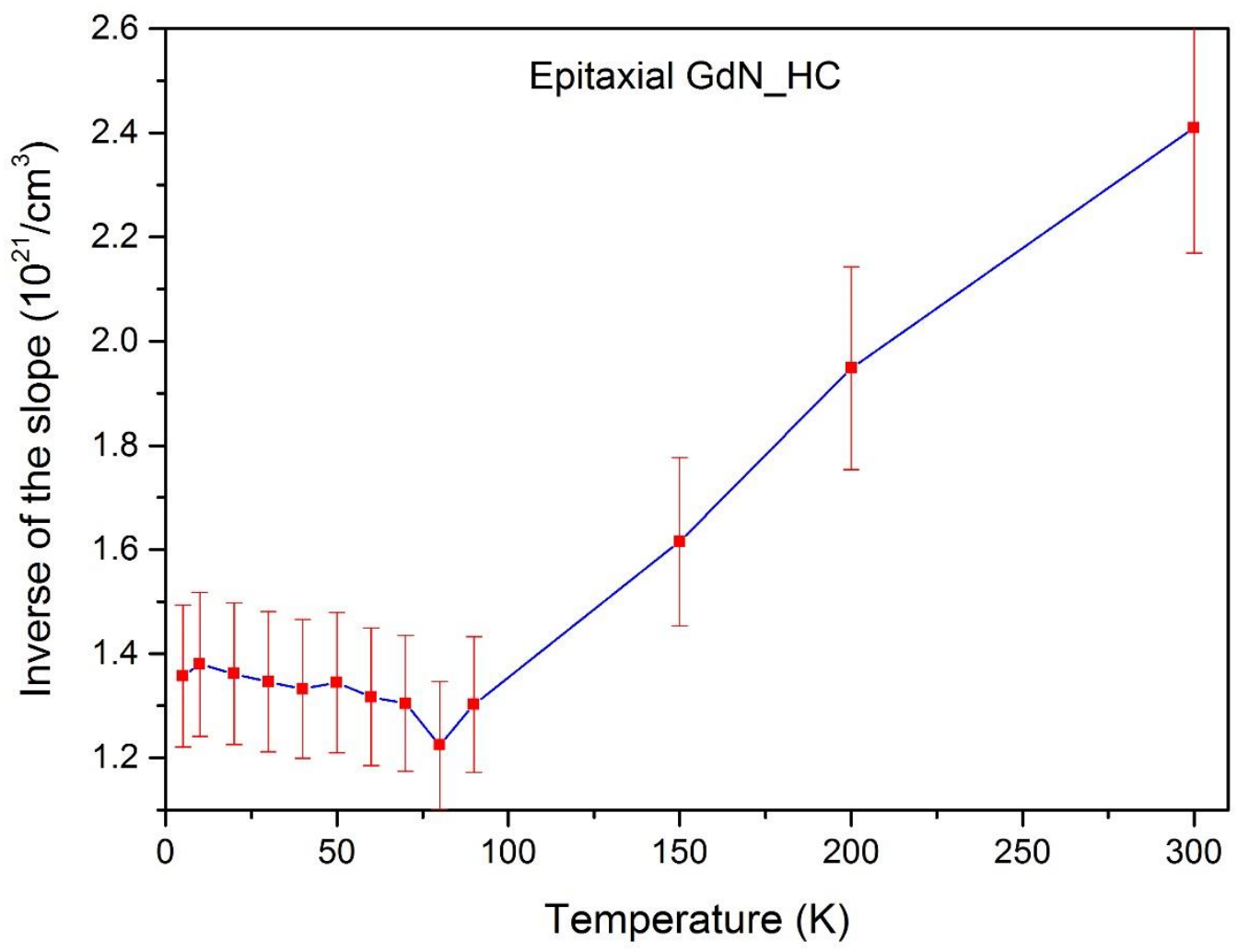

Figure 5.11: Inverse of the slope as a function of temperature retrieved from the Hall effect data. 
Figure 5.11 shows that the carrier concentrations which are typically large for this film and almost constant in the ferromagnetic state. On entering the paramagnetic phase there is no signature at all of a discontinuous change in the carrier concentration at the transition, rather there is a weak rise as temperature is increased. Such a high carrier concentration and very weak temperature dependence is characteristic of a material with the Fermi level lying inside the conduction band, with no Arrhenius excitation of charge carriers. The temperature dependence of the carrier concentration at the higher temperatures is somewhat surprising.

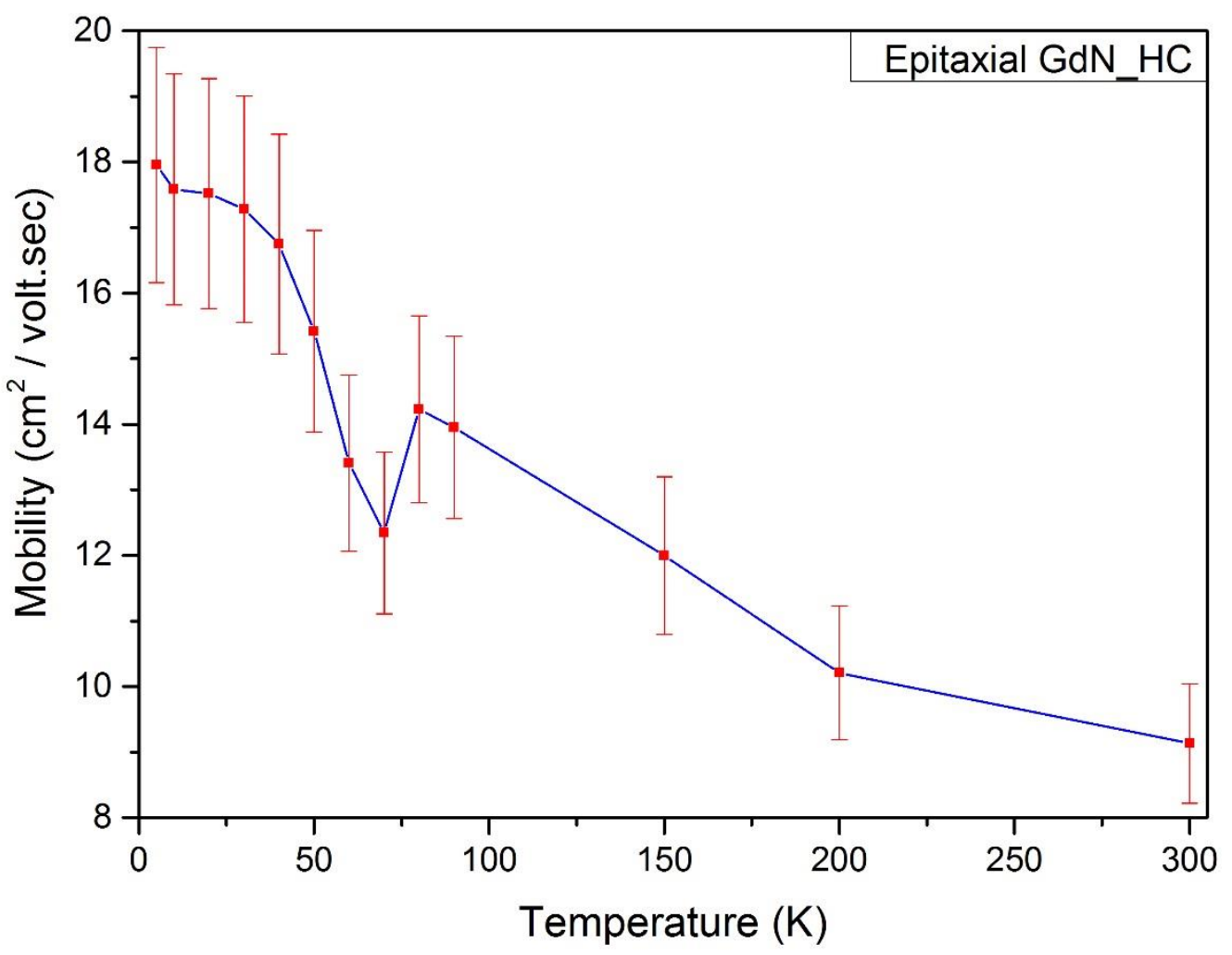

Figure 5.12: Mobility as a function of temperature of the epitaxial GdN_HC film.

A better understanding can come from looking at the mobility data which could expose the dominant mechanism for electron scattering that strongly influences the resistivity of the sample. Figure 5.12 illustrates the temperature dependent mobility data of the epitaxial GdN_HC film. The mobility is a respectable $18 \mathrm{~cm}^{2} \mathrm{~V}^{-1} \mathrm{~s}^{-1}$ at the lowest temperatures, suggesting an electron mean-free path of $10-30 \mathrm{~nm}$. It is evident that the mobility decreases as temperature increases towards ambient temperature and rapidly falls close to the Curie temperature, signalling the very strong contribution from $4 f$ spin-disorder scattering. The 
temperature dependence of the mobility well above $T_{c}$ likely results from electron-phonon scattering.

\subsubsection{Interpretation of Hall effect results}

The interpretation of Hall effect results is rather complicated for this film as we have not seen any previously reported GdN films which contain typically large carrier concentrations and interestingly they are also showing weakly temperature dependent characteristics in the paramagnetic region. As seen in Figure 5.11, the carrier concentration of this film is large, $\mathrm{n} \sim$ $2.4 \times 10^{21} \mathrm{~cm}^{-3}$ at room temperature, which reflects the larger nitrogen vacancy concentration occurring at the higher growth temperature. An estimate of the corresponding Fermi energy can be made based on the calculated band structure of $\mathrm{GdN}$, which yields $0.7 \mathrm{eV}$ for this sample, relative to the conduction band minimum. The film is clearly degenerately doped, so a conventional description in terms of impurity levels that result in a Fermi level lying in the band gap is clearly not applicable (e.g., figure 5.1). Instead carriers reside in the conduction band and the conductivity is dominated by mostly the usual single-band model as discussed more briefly in the following section.

We mentioned in the beginning of this chapter that the main source of carriers in GdN are nitrogen vacancies. Now we show that the transport properties are better explained by a model where each nitrogen vacancy contributes a level resonant with the conduction band, i.e. the relevant $V_{N}$ level lies above the conduction band minimum. It is interesting to see what this would mean for the carrier concentration and see how the carrier concentration could change with the temperature. 

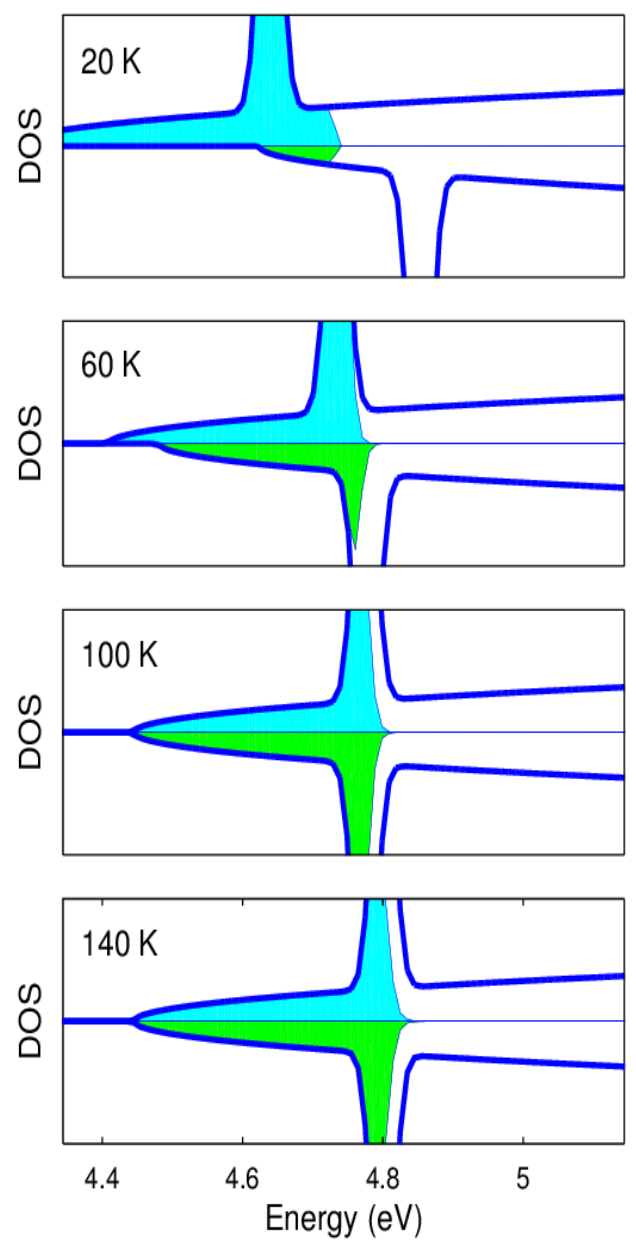
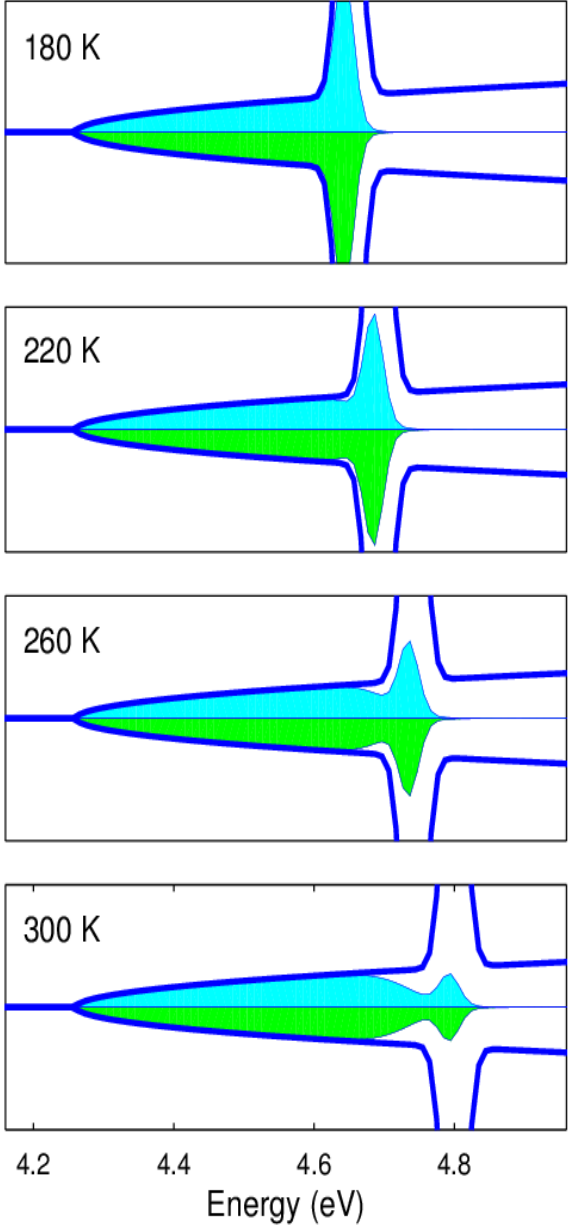

Figure 5.13: Schematic illustration of the density of states of $\mathrm{GdN}$ assuming an imprity level lying above the conduction band minimum. The shaded regions represent occupied states.

In Fig. 5.13 we sketch a pictorial representation of the spin-up and spin-down densities of states to visualise how the carrier concentration might change with temperature. The GdN conduction band is represented by a parabolic density of states, with a narrow feature sketched several tenths of an $\mathrm{eV}$ above the conduction band minimum representing an impurity state. This is consistent with the calculations of Punya et al. [173] which found some impurity states in the gap and a state inside the conduction band. For simplicity no impurity states have been included within the band gap in the schematic diagram. The shaded regions represent occupied states. The total density of impurity states has been chosen such that the Fermi level lies close to the resonant state, so almost all of the electrons are in delocalised conduction band states. This allows for the observed degenerate doping and high mobility. At low temperatures the bands spin-split so that conduction is dominated by only majority-spin electrons. This model can provide a speculative explanation for the temperature dependence of the carrier concentration 
if we assume that the energy of the resonant state is temperature dependent. If the resonant state moves closer to the conduction band minimum then it will capture electrons from the conduction band and decrease the carrier concentration.

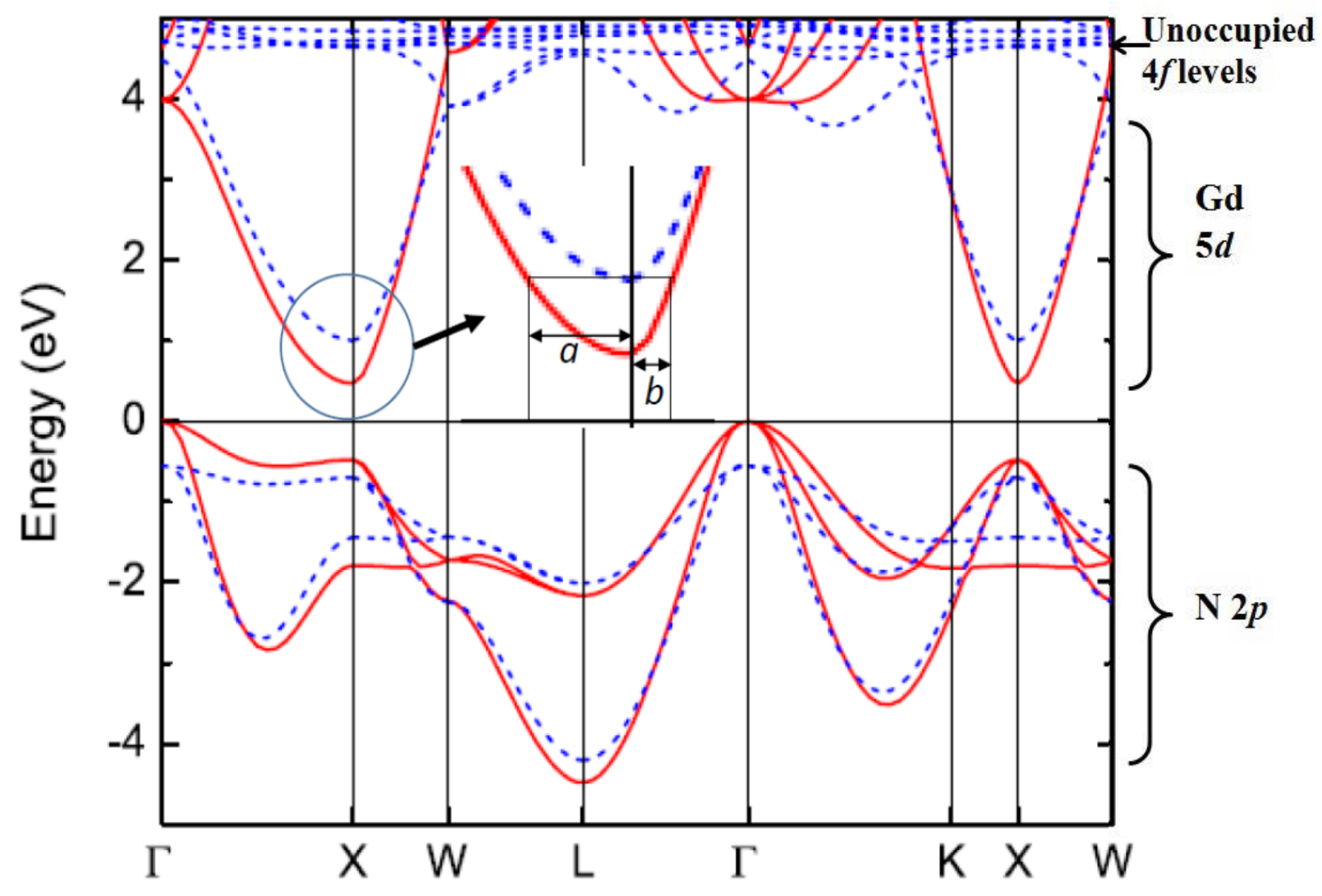

Figure 5.14: The band structure of GdN calculated within the LSDA $+U$ approximation [116].

The expanded view shows the axes of the prolate spheroid in the majority-spin conduction-band pocket. The solid lines represent majority-spin bands, and dashed lines the minority-spins.

We finish the discussion of the carrier concentration in this film with a simple calculation of the number of carriers that can exist before the minority-spin band becomes occupied. The LSDA $+U$ computed band structure for GdN shows (Figure 5.14) three prolate-spheroid electron pockets at the $X$ points, with a spin splitting of around $1 \mathrm{eV}[53,116]$. We have calculated the necessary carrier concentration to influence the conductivity by both channels, i.e. the carrier concentration at which the Fermi energy reaches the minority-spin conduction band. This is determined by calculating the volume of the majority-spin pockets at the Fermi energy corresponding to the bottom of the minority-spin pockets from Figure 5.14. Our calculation shows that the bands in Figure 5.14 can accommodate $3 \times 10^{21} \mathrm{~cm}^{-3}$ in each $X$-point 
pocket, so that the total majority-spin electron concentration in the three $X$-point pockets would be $\sim 10^{21} \mathrm{~cm}^{-3}$, which is in satisfactory agreement with the carrier concentration obtained from the Hall measurements.

\subsubsection{Thermopower results}

In order to get a more detailed picture of the electrical state of this GdN film, we have performed thermopower measurements on a film grown in a similar condition except using a different substrate. We cannot use silicon or sapphire substrates for the thermopower measurement because those substrates exhibit high thermal conductivity which introduces significant error in the thermopower measurement (as discussed in Chapter 4, Section 4.3.7). Measurements on substrate-supported films demand the utmost care to ensure that we measure the temperature difference along the substrate and between the two ends of the substrate. A good thermal layer needs to be put between substrate and $\mathrm{Cu}$ blocks and thermopower of known reference-lead must be used otherwise one would get spurious thermopower results. Thus we require a substrate which exhibits lower thermal conductivity but which is still suitable for epitaxial film growth. Our collaborator in France has grown a separate piece of GdN_HC film on a GaN substrate for conducting thermopower measurements [53]. 


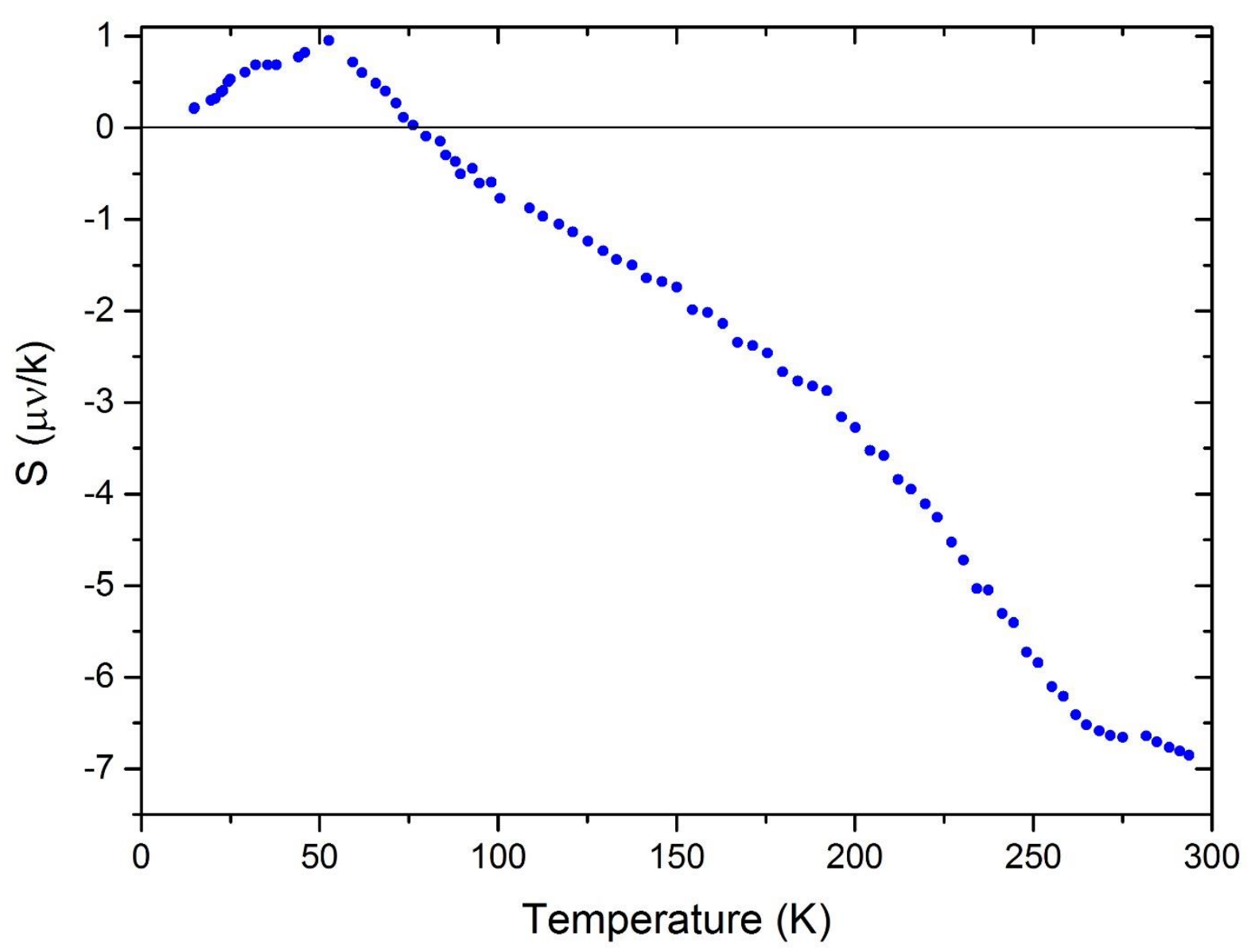

Figure 5.15: Temperature dependent thermopower of the epitaxial GdN_HC film.

Figure 5.15 depicts the temperature dependent thermopower results on the epitaxial GdN_HC film. The experiment was performed in the temperature range of $12 \mathrm{~K}$ to $300 \mathrm{~K}$. The thermopower data is presented here after correction for the reference leads which were manganin in our case [172]. The uncertainty of the measurement was $\pm 1 \mu \mathrm{v} / \mathrm{K}$ which mainly arose from the experimental procedures as discussed in Chapter 4, Section 4.3.7.

Figure 5.15 shows a negative thermopower in the paramagnetic phase, consistent with the sign of the charge carriers obtained from the Hall effect result. The magnitude of thermopower increase as temperature increases toward room temperature. 
Near $280 \mathrm{~K}$ a small bump appears. This arises from the measurement process where the sequence file has been tuned near this temperature to adjust the ramp rate (cooling and heating) of the film which could take some time to settle down to an equilibrium temperature prior recording the raw data. The standard practice for this experiment was to increase the ramp rate at high temperature to reduce the experiment time. In the present case, the automatic process was not completely synchronised with the LabView programming and temperature controller which leads to a small offset near RT.

The simplest model for the Seebeck coefficient in a degenerate electron gas (i.e. a metal or degenerately doped semiconductor) which shows a thermoelectric power is one that follows the well-known Mott formula [152 - 154, 171]

$$
S=\frac{\Pi^{2} k_{B}^{2} T}{3 e}\left(\frac{\partial(\ln \sigma)}{\partial E}\right)_{E_{F}}
$$

where, $k_{B}$ is the Boltzmann constant, $e$ is the electronic charge, $T$ is the absolute temperature and $\sigma$ is the electrical conductivity of the material as a function of the Fermi energy.

Equation 5.1 assumes that the parameters that determine the conductivity, i.e. carrier density and relaxation time, show no intrinsic temperature dependence, so that the increased diffusion at higher temperatures follows from the widening of the Fermi occupation number within $k_{B} T$ of the Fermi energy. It is common to argue that the energy scale over which the conductivity varies is given by the Fermi energy, e.g., $\sigma \sim \frac{\beta}{E_{F}}$, in which case Equation 5.1 reduces to

$$
S \sim \frac{\Pi^{2} k_{B}^{2} T}{3 e}\left(\frac{\beta}{E_{F}}\right)
$$

It is common to assume that $\beta=1$, and this provides an order of magnitude estimate for the Fermi energy:

$$
E_{F} \sim 284 \mu V K^{-1} \frac{k_{B} T}{S}
$$

The above Equation 5.3 guides us to obtain the diffusion thermopower slopes of $S$ from Figure 5.15. We obtain an average slope across the paramagnetic region of $\approx 3 \times 10^{-2} \mu V K^{-2}$, giving 
a Fermi energy of $\approx 0.8 \mathrm{eV}$ which again indicates the heavily conducting nature of the film where the Fermi level lies well above the bottom of the conduction band.

The thermopower decreases quite linearly below $55 \mathrm{~K}$ and approaches zero when temperature is reduced towards zero. A small positive peak is observed near $55 \mathrm{~K}$ and the thermopower changes sign near the transition temperature being positive down to the lowest temperature. Now we will shed light on this feature. The thermoelectric effect is driven by a temperature dependent diffusion of the charge carriers, which then accumulate at the location for which the diffusion is weaker. That is normally the colder end of the sample. Within this simple picture one can immediately observe that at higher temperature more charge carriers diffuse from the high temperature region and accumulate at the cold end of the sample, leading to the negative Seebeck coefficient. In the ferromagnetic region, below $T_{c}$ the mobility is limited by spin disorder, imposing a temperature dependent relaxation time. In this case the relaxation time decreases as the temperature rises toward $T_{c}$, which we have exactly seen in our mobility data. Thus in this situation the electron diffusion is higher at lower temperatures, leading to the observed positive Seebeck coefficient.

We have presented the first thermoelectric results of the epitaxial GdN_HC film. A high Fermi energy is estimated from the diffusion thermopower slope which is in agreement with the Fermi energy approximately calculated from the density of states and measured carrier concentration. Both values suggest that the Fermi level is well inside the conduction band, which suggests the film is a degenerately doped semiconductor showing metallic characteristics [53]. This finding contrasts with the most commonly identified semiconducting GdN films where the Fermi level lies below the conduction band. 


\subsection{Summary and Conclusions}

This chapter reports measurements of magnetic and electron transport phenomena in the intrinsic ferromagnetic semiconductor $\mathrm{GdN}$ doped with $10^{21} \mathrm{~cm}^{-3}$ electrons. The magnetic measurements found that the transition temperature and saturation magnetisation are $65.5 \pm 1$ $\mathrm{K}$ and $6.5 \pm 0.5 \mu_{\mathrm{B}} / \mathrm{Gd}^{3+}$ respectively, which are close to the most reported values in the literature. Near the transition temperature magnetic fluctuations play an important role in dominating the electron scattering of the film which then changes the resistance of the film as seen in the magnetoresistance results.

The conductivity, carrier concentration and thermoelectric power have been measured from 5 to $300 \mathrm{~K}$. The resistivity of this film is very low because of the high carrier concentration, as measured from the Hall effect. The carrier concentration shows a weak temperature dependence in the paramagnetic region. The results below the Curie temperature are in agreement with transport dominated by the majority-spin pockets in the conduction band of GdN. At the lowest temperatures the mobility is $18 \mathrm{~cm}^{2} \mathrm{~V}^{-1} \mathrm{~s}^{-1}$, but shows a rapid reduction as spin-disorder scattering set in near $T_{c}$

For the first time thermopower measurements on $\mathrm{GdN}$ have been reported. The thermoelectric power is positive in the ferromagnetic state, where spin-disorder scattering imposes a strong temperature dependent reduction of electron diffusion at higher temperature. It changes sign across the ferromagnetic-paramagnetic phase transition, afterward sharing the negative sign of the carriers as expected for an electron diffusivity that increases with temperature. Furthermore, its magnitude in the paramagnetic phase is in agreement with the Fermi energy for such a heavily doped film.

The overall picture that emerges is of a film doped such that the Fermi level is well inside the conduction band. This is not consistent with doping induced by states inside the band gap, and instead implies that the nitrogen vacancies must introduce states well up inside the conduction band. This picture goes beyond previous descriptions of experimental results that have focussed on in-gap states, but it is closer to the picture that emerges from theoretical calculations of the electronic band structure of $\mathrm{GdN}$ with nitrogen vacancies. 


\section{Chapter 6}

\section{Magnetotransport properties of low carrier concentration epitaxial gadolinium nitride film (GdN_LC)}

\subsection{Introduction}

In this chapter we will present the magnetic and transport results of an epitaxial GdN film which exhibits lower carrier concentration than the previous epitaxial film as discussed in the earlier Chapter 5. Throughout this chapter we will mostly mention this film as a GdN_LC film, where the term 'LC' stands for the lower carrier concentration. This chapter has four main Sections, i.e. 6.2, 6.3, 6.4 and 6.5 respectively, where magnetic results have been discussed in Section 6.2. Section 6.3 presents the resistivity and magnetoresistance results and later Section 6.4 comprised Hall effect results. Note that transport results are limited to resistivity, magnetoresistance and Hall effect results. We do not have any thermopower results of this film as the film was broken before such measurements could be made.

We note that this film required an innovative Hall effect interpretation. The Hall effect data interpreted with the help of magnetic data where experimental SQUID data helps us to understand the anomalous Hall effect in the ferromagnetic region. In the paramagnetic phase there was still a contribution from the anomalous Hall effect for which we had to use the Brillouin function in the fitting procedure. Under the Hall effect Section 6.4, we put a separate subsection 6.4.1 to discuss the fitting procedure in the paramagnetic and ferromagnetic regions, respectively. Data fitting in the ferromagnetic region is compromised with the shape anisotropic effect which needs to be considered while we utilise the SQUID data. The 
interpretation of Hall effect results has been presented under the subsection 6.4.2. We have summarised the entire results of this chapter in the last Section 6.5.

The primary intention of this chapter is to understand the transport properties of $\mathrm{GdN}$ film in terms of carrier concentration which is influenced by the nitrogen vacancies as we change the growth temperature. Therefore, in the present case we have only changed the growth temperature to control the nitrogen vacancies and stick with the rest of the other growth parameters as used for the high carrier concentration GdN_HC film. This epitaxial GdN_LC film was grown at temperature $\left(650^{\circ} \mathrm{C}\right)$ by the molecular beam epitaxy (MBE) method using ammonia $\left(\mathrm{NH}_{3}\right)$ as the nitrogen source. Similar to the other epitaxial GdN_HC film, the $120 \pm$ $10 \mathrm{~nm}$ thick epitaxial GdN_LC film was deposited on a $50 \mathrm{~nm}$ thick AlN template on a silicon substrate in the MBE laboratory at Valbonne, France.

\subsection{Magnetic results of the GdN_LC film}

Similar to the previous epitaxial GdN film, we have performed the magnetic measurements of this epitaxial GdN film from $2 \mathrm{~K}$ to $300 \mathrm{~K}$, in the presence of a 250 Oe magnetic field. The magnetic measurements were carried out at the Robinson Research Institute, Gracefield, using the Quantum Design SQUID magnetometer. During the experiment the magnetic field was applied in the in-plane direction, i.e. magnetic field applied parallel to the film surface. We would like to note here that the magnetic results of this film are pretty much similar to the previous epitaxial film. Therefore, here we will only highlight the key magnetic results of this film. 
Figure 6.1 shows the magnetisation as a function of temperature. As temperature reduces below $65 \mathrm{~K}$, the magnetisation curve changes the behaviour and shows an upturning characteristic as expected. This feature suggested the ferromagnetic Curie temperature is in this region which is obtained from the Curie-Weiss fitting as shown in the inset. The Curie-Weiss plot indicates the film exhibits the ferromagnetic nature below the $64.5 \pm 1 \mathrm{~K}$ temperature which is around 5 $\mathrm{K}$ lower than the usually reported transition temperature of $70 \mathrm{~K}[24,79,81]$ and $1 \mathrm{~K}$ lower than the GdN_HC film. Interestingly, we have observed the anomaly behaviour of the magnetisation curve near $50 \mathrm{~K}$ which has been reported but rarely seen. This feature is apparently showing the double transition temperature as seen in the previous research [97] and interpreted as signalling magnetic polarons.

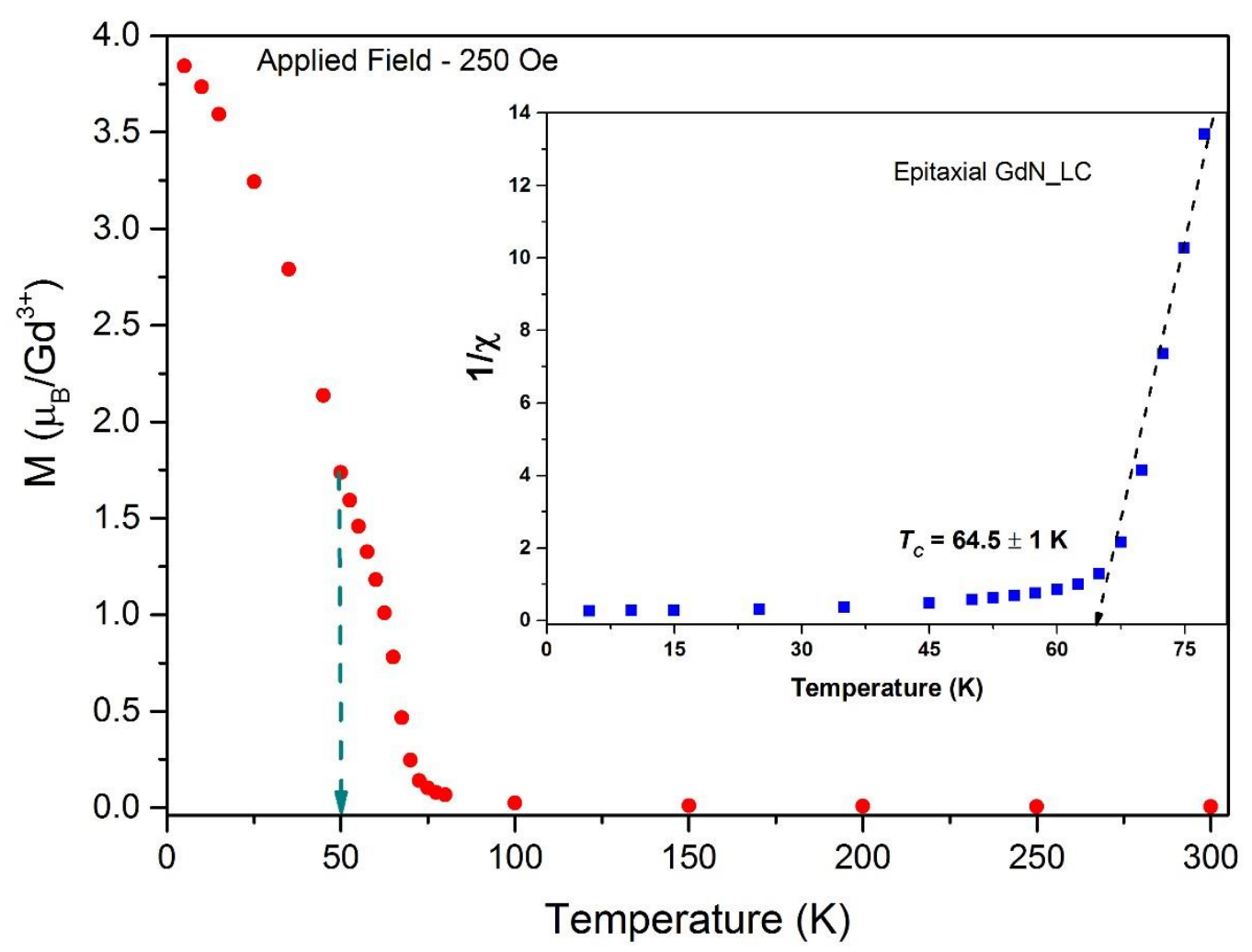

Figure 6.1: Magnetisation curve and Curie-Weiss fitting (inset) of the epitaxial GdN_LC film as a function of temperature. 
We have plotted the magnetisation as a function of the field in Figure 6.2. The magnetisation of the film rises steeply in the presence of lower field and then saturates at the high magnetic field. This result is expected as the magnetisation arises from the $4 f$ electrons which orient in a similar direction to the applied magnetic field and leads to the saturation magnetisation. The $10 \mathrm{~K}$ saturation magnetisation of this film is found to be $7 \pm 0.5 \mu_{\mathrm{B}} / \mathrm{Gd}^{3+}$ which is most commonly reported in the literature $[108,110]$. In the next two sections we will utilise these magnetic results to interpret the transport results of this film.

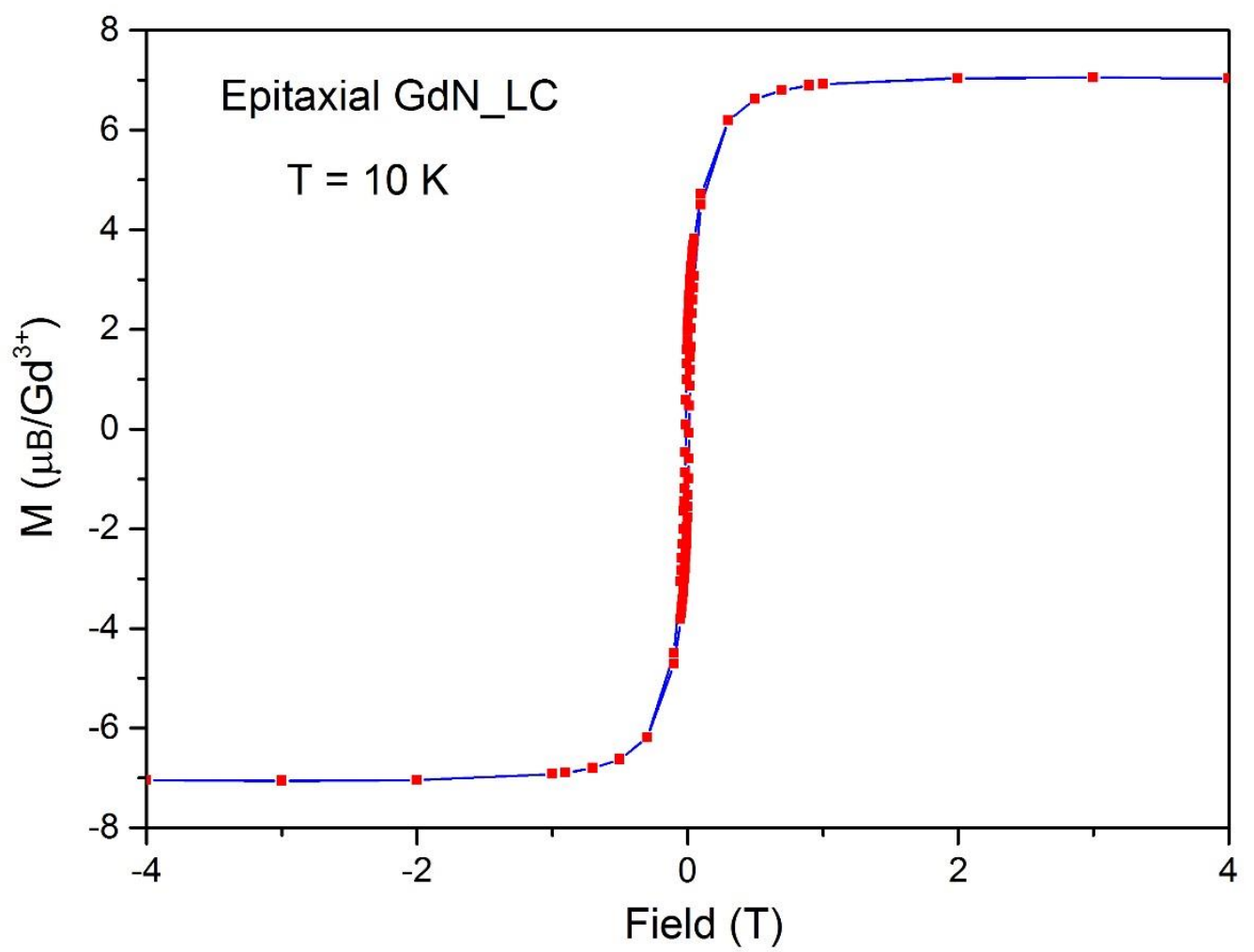

Figure 6.2: Magnetisation data of the epitaxial GdN_LC film as a function of field at $10 \mathrm{~K}$. 


\subsection{Resistivity and magnetoresistance results}

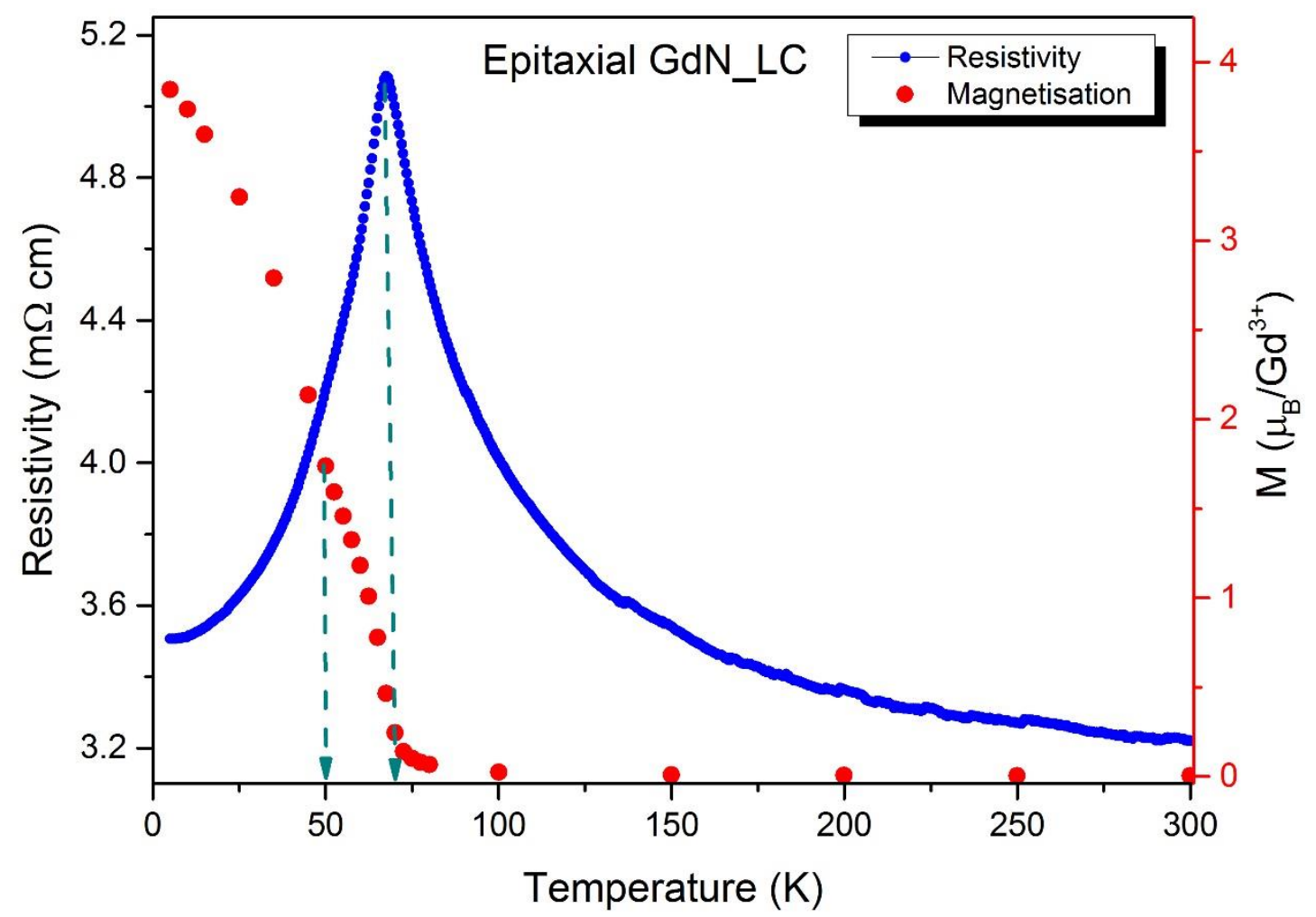

Figure 6.3: Temperature dependent resistivity and magnetisation of the epitaxial GdN_LC film.

The resistivity and magnetisation as a function of temperature of this film were performed from $2 \mathrm{~K}$ to $300 \mathrm{~K}$ temperature region and presented in Figure 6.3. The lowest resistivity of this sample at room temperature is $3.25 \mathrm{~m} \Omega \mathrm{cm}$ which is a factor of 10 higher than the previous epitaxial GdN_HC film. As the temperature decreases from ambient temperature the resistivity of this sample increases and reaches a maximum around $64.5 \pm 1 \mathrm{~K}$, then drops rapidly at lower temperatures, giving a positive temperature coefficient of resistivity as expected for a resistivity dominated by the magnetic disorder scattering. 


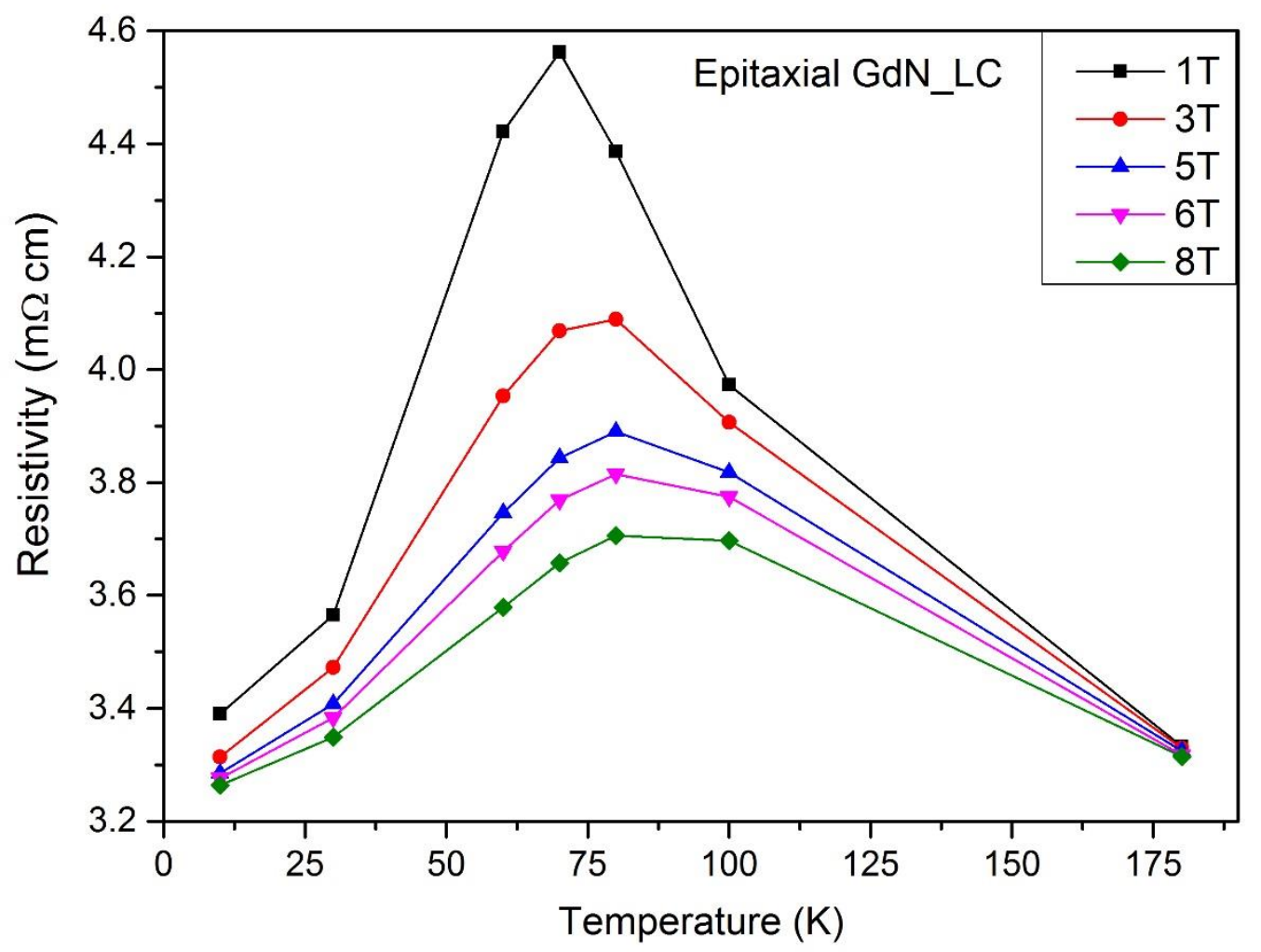

Figure 6.4: Temperature and field dependent resistivity of the epitaxial GdN_LC film.

As seen also in the GdN_HC film of Chapter 5, the resistivity peak at $T_{c}$ is strongly related to the magnetic disorder [53] which is clearly signalled also in our magnetoresistance results as shown in Figure 6.4. The strong resistivity peak is seen near the transition temperature where magnetic disorder reduces significantly as the strength of the external magnetic field increases. The figure shows that in the presence of the highest magnetic field of $8 \mathrm{~T}$ the resistivity reduces by around $25 \%$ due to the reduction of magnetic disorder scattering. 
To further support this we have compared the magnetic disorder of both epitaxial films as shown in Figure 6.5 at $30 \mathrm{~K}$. The extensive study allows a magnetization-magnetoresistance comparison, revealing a clear correlation between the magnetization deficit $\left[M_{S a t}-M(\vec{B}, T)\right]$ and magnetic disorder scattering.

It is significant that the MR is small at fields below $\sim 1 \mathrm{~T}$, the field range at which the domains grow rapidly without significantly affecting the short-range disorder. At larger fields the two measures are colinear within uncertainty, suggesting that indeed the major source of the magnetisation deficit at these fields is related to short-range disorder. Thus at higher fields, $\geq$ $1 \mathrm{~T}$, the magnetic disorder at the scale of a few interatomic distances is reduced by the Zeeman interaction with the $4 f$ elecrons on $\mathrm{Gd}^{3+}$ sites, reducing in turn the resistivity.

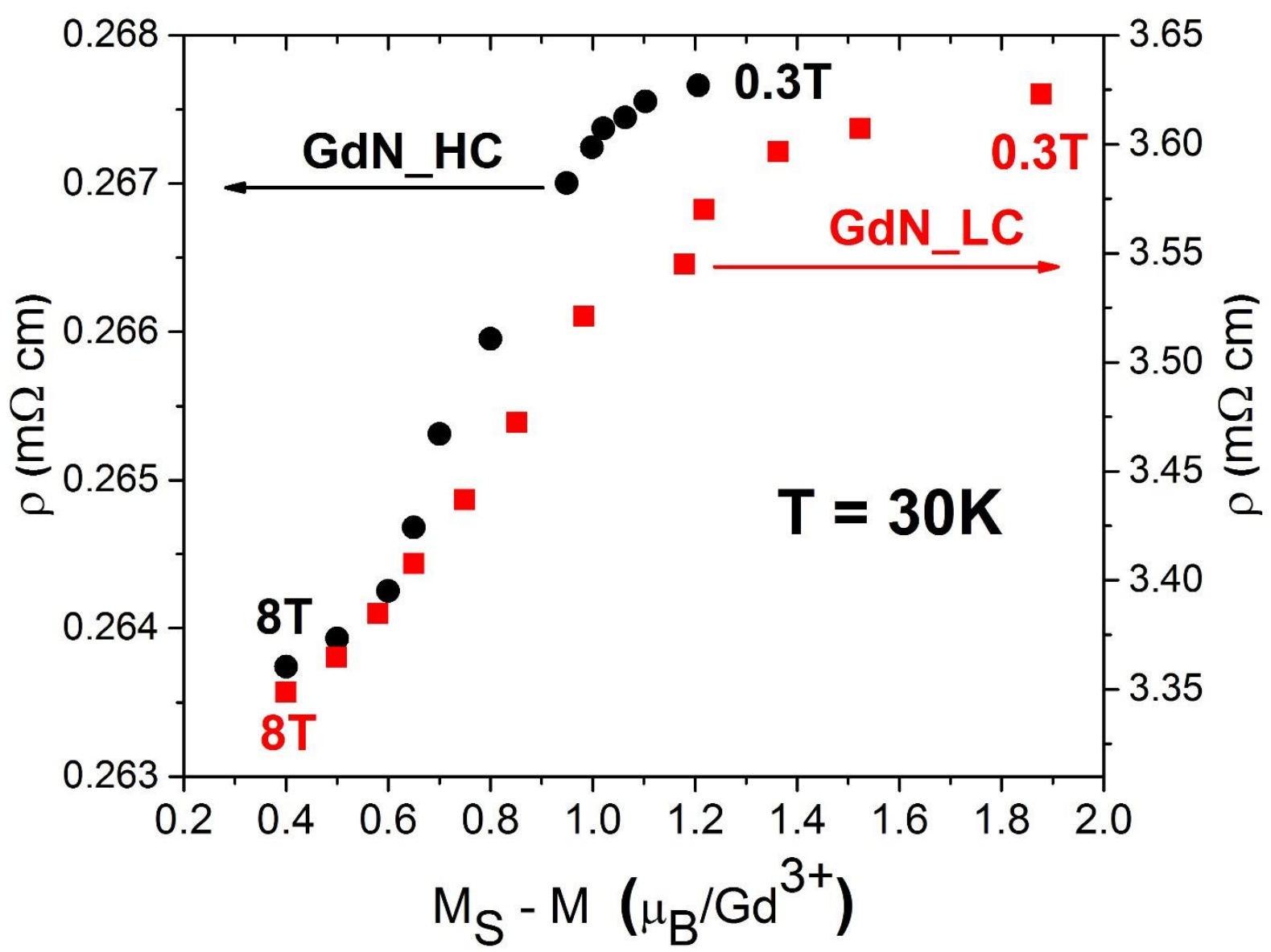

Figure 6.5: The resistivity of the two epitaxial films at the intermediate temperature of $30 \mathrm{~K}$ plotted vs the departure of the magnetisation from its saturation value. 


\subsection{Hall effect results}

In order to establish more details about the transport properties we have also measured the Hall effect at different temperatures. The experiment was performed from $300 \mathrm{~K}$ down to $5 \mathrm{~K}$ with varying magnetic fields. Figure 6.6 shows the Hall resistance of the epitaxial GdN_LC film as a function of the applied field at various temperatures. The Hall resistance shows a negative slope, suggesting charge carriers are electrons, as expected for carriers which originate from nitrogen vacancies.

At room temperature, the Hall resistance $\left(R_{H}\right)$ slope is approximately linear, and the slope gives the carrier concentration of $5.7 \pm 0.5 \times 10^{20} / \mathrm{cm}^{3}$. Once we reduce the temperature from $300 \mathrm{~K}$ to $100 \mathrm{~K}$, the slope becomes larger which might be interpreted as a reduction of carrier concentration. But below we would argue that this is rather a matter of contribution of an anomalous Hall effect (AHE) or an extraordinary Hall effect (EHE). Below the $T_{c}$ we observed an offset in the plotted Hall resistance as a function of field line; this was an anomalous Hall effect (AHE) which is proportional to the magnetisation of the film and saturated at higher field above the 2.1 T because of the shape anisotropy as mentioned earlier (Chapter 2, Section 2.1.8).

Earlier Hall effect experiments [15, 24] simply focused on obtaining the carrier concentrations from the inverse of the high field slope and probably missed the opportunity to find any significant relationship between magnetisation of the film and the Hall effect results. Technically, the ignorance of the influence of the magnetisation on slopes must provide the pseudo carrier concentration which then lead to a wrong interpretation of transport results. For the first time we have successfully identified that the Hall effect slopes also affected by the magnetisation of the film. Therefore, the interpretation of results are performed utilising the magnetisation results of this film and presented in Sections 6.4.2. 
But, before we present the interpretation of the Hall effect results, it is necessary to understand how we fit the data in the ferromagnetic and paramagnetic regions to eliminate the contribution of magnetisation which has been discussed briefly in the next section.

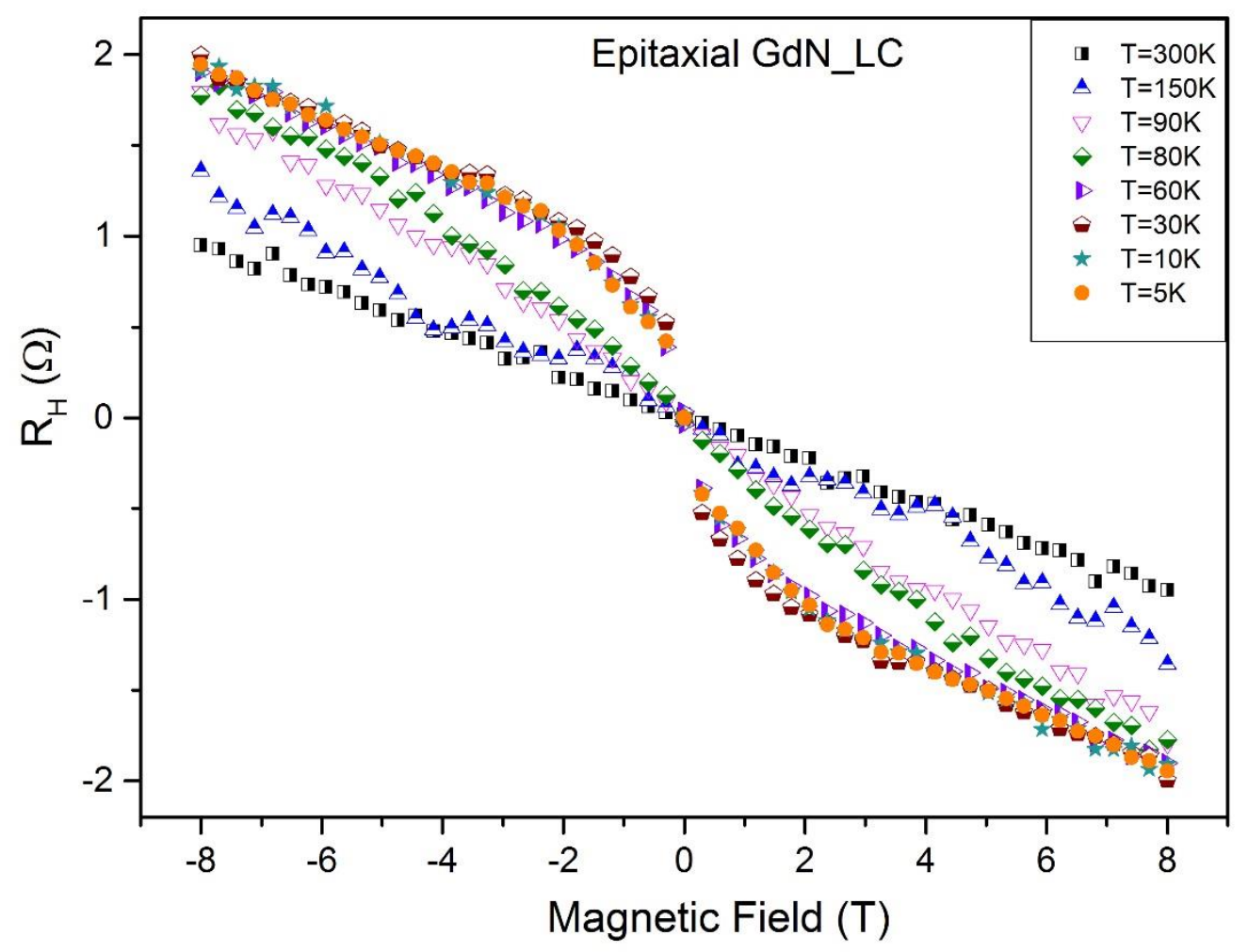

Figure 6.6: Hall resistance as a function of applied magnetic field for the epitaxial GdN_LC film.

\subsubsection{Model to fit the Hall effect data}

The interpretation of Hall effect data of this sample is undoubtly complicated. As we indicated earlier, magnetisation is significant in the paramagnetic region, so that an anomalous Hall contribution enhances the slope and can lead to an underestimate of carrier concentration. Therefore, a careful correction is required to obtain the actual carrier concentration. To tackle this anomaly we have considered a phenomenological model to explain the observed anomaly in Hall effect results. 
The following paragraphs will give us guidance to deal with the magnetisation of the film at various temperatures. We have recalled a few terms here from Chapter 2 for a better explanation. To further elaborate our discussion, we will first express the simple equation for the Hall resistance of magnetic materials, which can be written as:

$$
\rho_{x y}=R_{H} \vec{B}+R_{A} M(T, \vec{B})
$$

The term $R_{H}=(-1 / n e)$, represents the ordinary Hall effect, which is proportional to the magnetic field $\vec{B}$ and inversely proportional to the number of charge carriers in the sample. The term $R_{A}$ of the right-hand side stands for an anomalous Hall effect, which is proportional to the magnetisation of the sample. Below $T_{c}$ we utilised the experimental SQUID results to obtain the value of $M(T, \vec{B})$. In the paramagnetic phase there was still remanent magnetisation prominent, thus we have used the Brillouin function to estimate the value of $M(T, \vec{B})$ in this region. The term $R_{A}$, plays a very significant role towards the results and it represents the amplitude of anomalous Hall effect and found to be temperature dependent in our result. So, the total Hall effect has a contribution of normal Hall effect plus the anomalous Hall effect, which is large below the $T_{c}$ and has a significant effect at lower temperatures. The above discussion emphasizes that the data need to be treated separately in the ferromagnetic and paramagnetic regions to obtain the correct carrier concentration.

\subsubsection{Fitting in the ferromagnetic region}

The experimental result shows, the high field Hall resistance slopes [Figure 6.6] at $30 \mathrm{~K}$ and $10 \mathrm{~K}$ are found to be nearly the same and the carrier concentration does not depend on temperature. This gives the constant carrier concentration. The extrapolation to zero gives the amplitude of anomalous Hall effect and we find that it does depend on temperature. As noted earlier in Chapter 5, Section 5.2.3 (GdN_HC), the slope of high field in that film gives the carrier concentration and it turned out amazingly to be the same at $30 \mathrm{~K}$ and $10 \mathrm{~K}$. 
In the ferromagnetic region the anomalous Hall effect seen in Figure 6.6 is prominent which follows the magnetisation. We will see this evidence once we fit the Hall effect data in the ferromagnetic region using the SQUID data. It is to be noted that, the SQUID measurement was performed for the in-plane direction, i.e. the magnetic field was applied parallel to the film's surface whereas for the Hall effect measurement the field was applied in the perpendicular direction to the film's surface. The saturation field in the field normal Hall effect configuration is dominated by the shape anisotropy, which requires a field around $2.1 \mathrm{~T}(4 \pi M)$ to show the saturation magnetisation as stated earlier [53]. Therefore, we have considered the value of magnetisation in the out-of-plane direction for the low temperature Hall effect data fitting. Figure 6.7 shows the SQUID results in the in-plane direction whereas dashed line is the pictorial representation of SQUID data in the out-of-plane orientation.

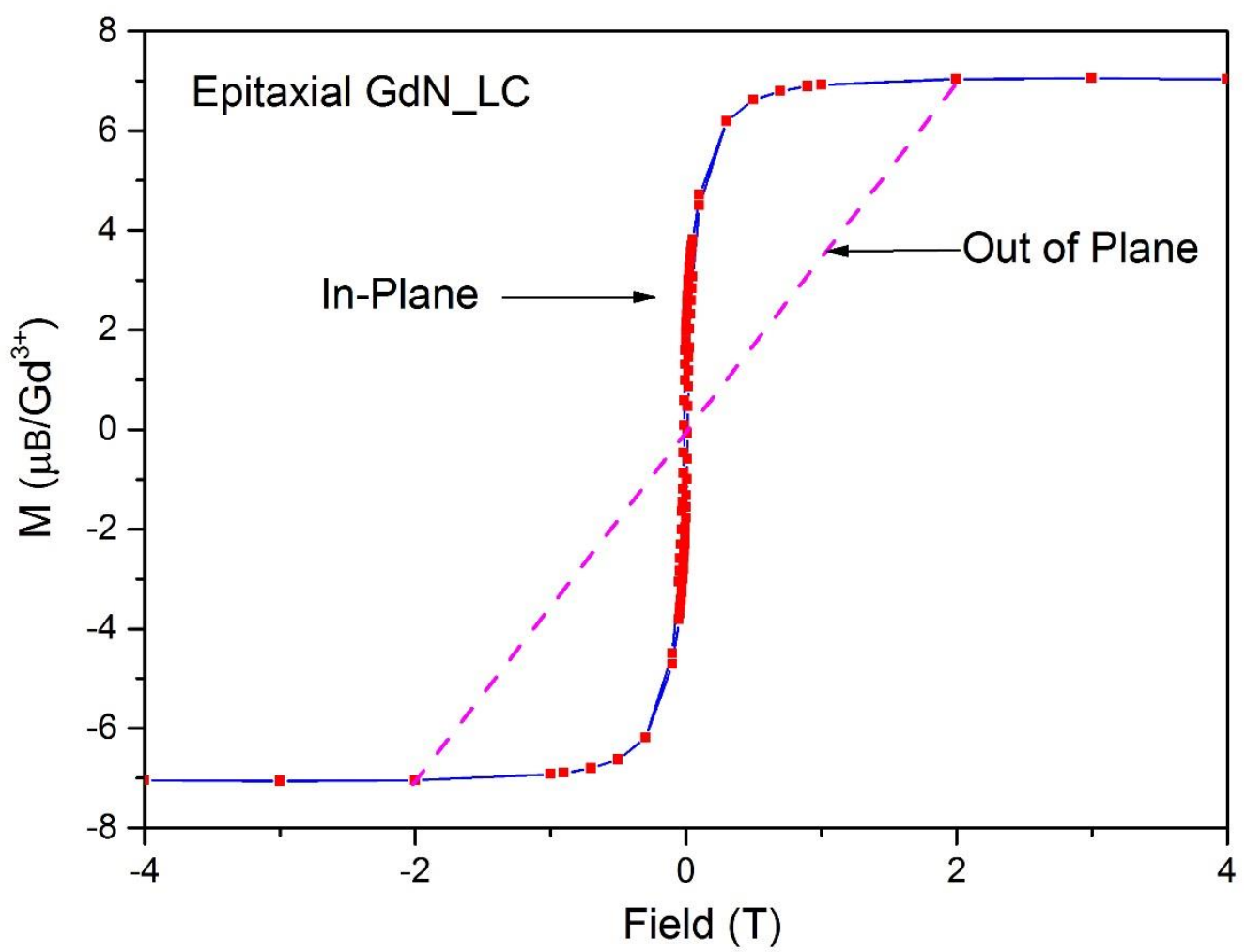

Figure 6.7: In-plane and out-of-plane magnetic data for the shape anisotropy correction. 
To further elucidate Equation 6.1 has been used to fit the low temperature data and a satisfactory fit was observed. Figure 6.8 shows a low temperature fitting for data collected at $10 \mathrm{~K}$. The inset figure shows the fitting at $30 \mathrm{~K}$. There are two parts involved in the fitting process, i.e. fitting in the high field slope (Normal Hall effect $\left(R_{H}\right)$ - refer to Equation 6.1) and fitting in the low field slope (Anomalous Hall effect $\left(R_{A}\right)$ - refer to Equation 6.1).

The term $(-1 / n e)$ was kept constant for both temperatures $(30 \mathrm{~K}$ and $10 \mathrm{~K})$ whereas the amplitude of the anomalous Hall effect " $R_{A}$ " was found to be temperature responsive in order to match the fitting result with the experimental data. Our calculated results fit reasonably well with the experimental result which certainly indicates that the carrier concentration of this film is constant at low temperatures.

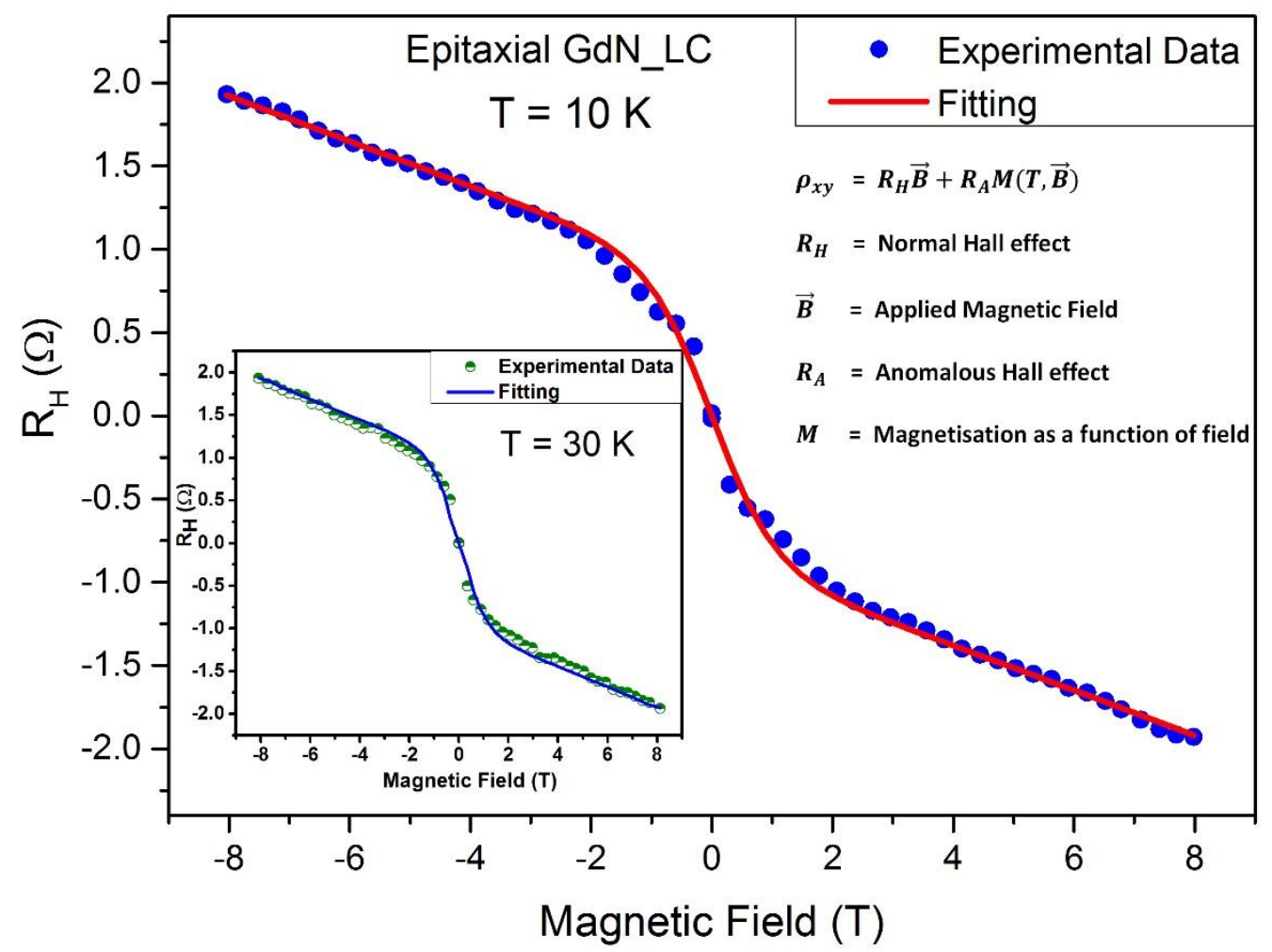

Figure 6.8: Experimental and fitting data of Hall resistance as a function of field at $10 \mathrm{~K}$ and $30 \mathrm{~K}$ (inset). 
It is important to note that the strength of the anomalous Hall effect (value of $R_{A}$ ) changes at the lowest temperatures which can be attributed to the mobility and scattering of the charge carriers [138]. We have seen in our data that the strength of the anomalous Hall effect has changed a lot even when the carrier concentration is almost the same. For example, the strength of the anomalous Hall effect (value of $\left.R_{A}\right)$ for $30 \mathrm{~K}\left(R_{A}=-0.260 \pm 0.013\right)$ is much larger than the $10 \mathrm{~K}\left(R_{A}=-0.170 \pm 0.008\right)$ data. The uncertainty in the strength of AHE mainly comes from how accurately we estimate slopes from the experimental data. We had taken the best fit and least fit of slopes and then considered the average value of slopes which is accurate within the 5\% uncertainty. The difference of the strength of AHE is quite substantial and clearly above the noise. This is really interesting to understand but at present we do not have any clear explanation. We could speculate from our experimental result that there might be a significant relationship between mobility and strength of the anomalous Hall effect which needs to be investigated thoroughly to get on top of this.

\subsubsection{Fitting in the paramagnetic region}

We now consider the paramagnetic region. Here one would see in the data (Figure 6.6) above $60 \mathrm{~K}$, the slope appears to increase as we decrease the temperature. Our first interpretation is that there is a temperature dependent carrier concentration, but then it is identified that there is a potential contribution of the anomalous Hall effect to the slope. In this section we will argue that the changes of apparent carrier concentration are quite likely associated with the magnetisation of the film. In order to discuss that one needs to know what is the temperature and field dependence magnetisation of the sample. We do not have a complete set of magnetisation data for that but we can rely on the Curie-Weiss law approach. To see how the magnetisation data of this film might look, we show the Curie-Weiss model magnetisation in the Figure 6.9 as a function of temperature and field. And one can see that by increasing the magnetic field, one gets a significant increase in the magnetisation as the magnetic field reduces the magnetic fluctuation. We have obtained the value of magnetisation for the selected temperatures in the paramagnetic region, i.e. $T=150 \mathrm{~K}, 90 \mathrm{~K}$ and $80 \mathrm{~K}$ for using our fitting process.

The model we use assumes that the magnetisation follows the modified Brillouin function or the Curie Weiss function which is used in the ferromagnetic phase by replacing the temperature with $T-T_{c}$, instead of $T$ and we now model such a system. 


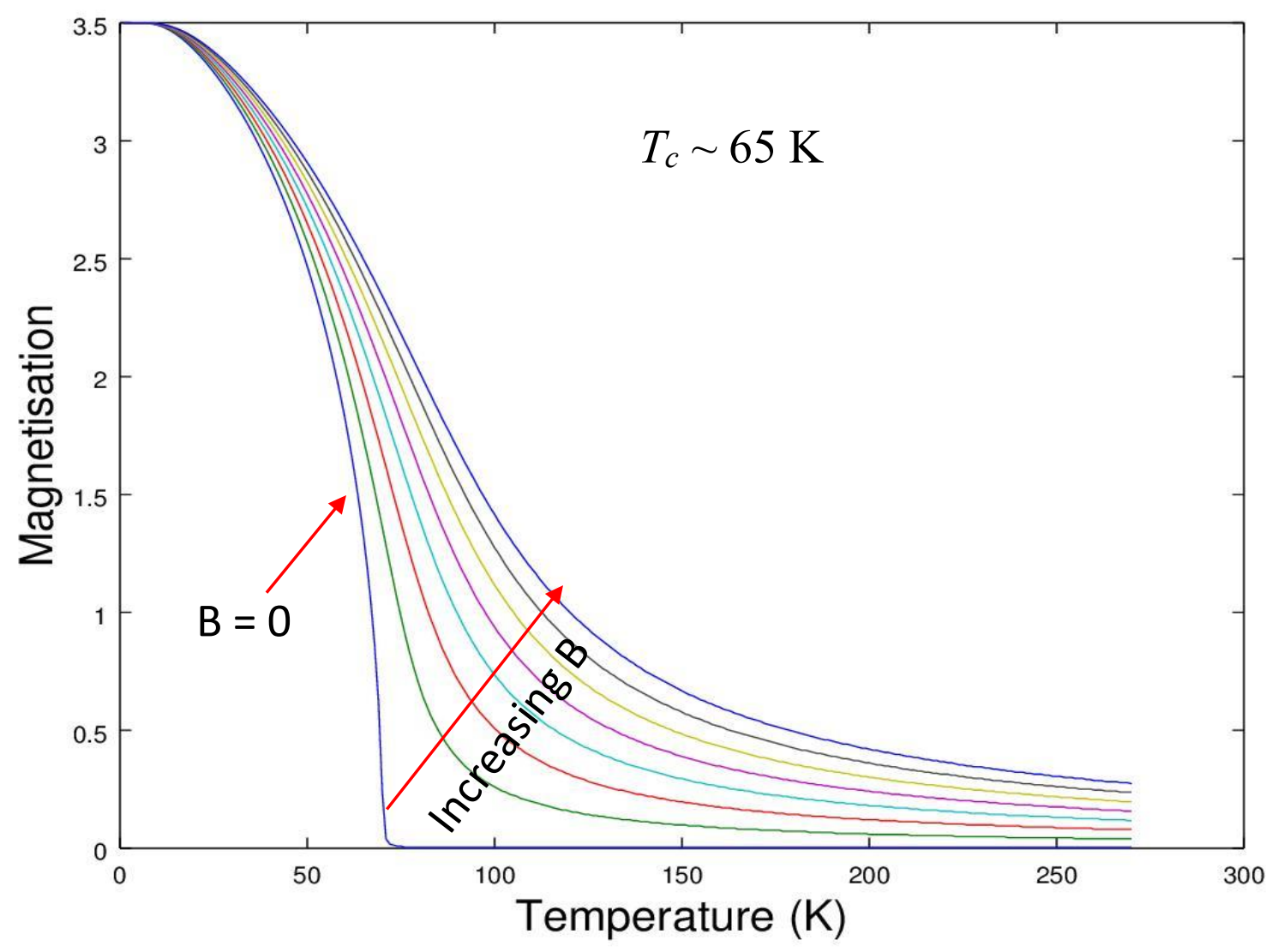

Figure 6.9: Estimated magnetisation as a function of temperature and field for the epitaxial GdN_LC film. 
Figure 6.10 shows the comparison of experimental and fitted Hall resistance data plotted as a function of field for selected temperatures. Equation 6.1 has been used to fit the calculated data with the experimental Hall effect data. Unlike the ferromagnetic region, the process is a bit complicated here which we tried to explain step by step in a simple manner. Firstly, it is assumed that the slope at $300 \mathrm{~K}$ is least affected by the magnetisation of the film; it is then worthwhile to compare how at the other temperatures the slope is affected by the magnetisation of the film.

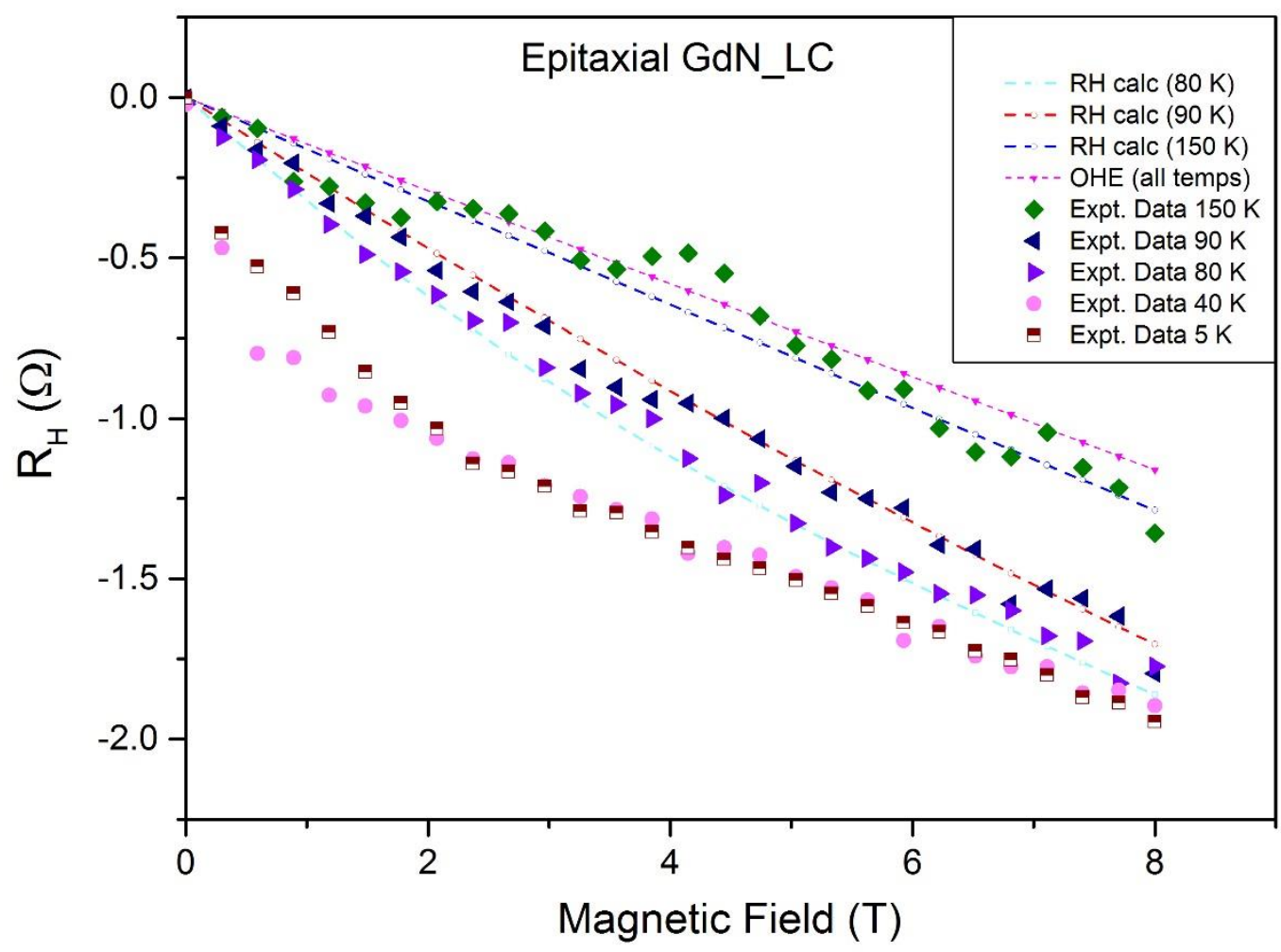

Figure 6.10: Comparison of experimental and fitted Hall data of the epitaxial GdN_LC film in the paramagnetic region. 
To understand this, we have started playing with three different temperatures, i.e. $150 \mathrm{~K}, 90 \mathrm{~K}$, $80 \mathrm{~K}$ in the paramagnetic region. During the fitting process, we kept constant the Hall resistance data of $300 \mathrm{~K}$ for all three temperatures and then added this data (dashed pink line) with the estimated magnetisation of each selected temperature multiplied by the strength of anomalous Hall effect " $R_{A}$ ". If data fits well then it clearly signals that variation of high field slopes at different temperatures is observed due to the changes of the magnetisation of the film.

To make it clearer, here we will present an example of data fitting for one temperature, i.e. 150 $\mathrm{K}$. We know the value of magnetisation as a function of field at $150 \mathrm{~K}$ from Figure 6.9. Then we multiplied the magnetisation $(M)$ with the strength of anomalous Hall effect which is " $R_{A}$ ". Finally, we add the product of $M \times R_{A}$ with the ordinary Hall resistance data of $300 \mathrm{~K}$. The fit was performed by adjusting the value of " $R_{A}$ " until the calculated Hall data matched the measured data of this epitaxial GdN_LC film. The most important thing to note here that, we have not added the Hall resistance data of $150 \mathrm{~K}$ with the value of $M \times R_{A}$ in the fitting process, instead $300 \mathrm{~K}$ Hall resistance data was added. The carrier concentration at $150 \mathrm{~K}, 90 \mathrm{~K}$ and 80 $\mathrm{K}$ were then obtained from the linear part of the high field that also matches the carrier concentration at room temperature. A reasonably good fitting of the higher temperature data indicates the strength of the slope varies due to the magnetisation of the sample. The carrier concentration is assumed constant in the paramagnetic region similar to the ferromagnetic region and they are temperature independent which we will discuss briefly in the interpretation section.

Now one would be curious to know why this model has not been utilised for the other epitaxial GdN_HC film. As we know the Hall resistance has two components, i.e. ordinary Hall effect and Anomalous Hall effect (AHE). We estimate the carrier concentration from the high field slope of the ordinary Hall effect. Because the magnetisation becomes saturated at the high field $($ for $\operatorname{GdN}=(4 \pi M)=2.1 \mathrm{~T})$.

For GdN_LC film, the high field Hall resistance slope for $300 \mathrm{~K}$ and $5 \mathrm{~K}$ look similar, which suggest the carrier concentration are the same at these two temperatures. At high temperature magnetisation is zero and no obvious contribution comes from the AHE. At low temperature ( $5 \mathrm{~K})$ magnetisation has a constant value and it is saturated, so it just adds a constant AHE and does not change the slope of Hall resistance. 
But in the intermediate temperature region, especially near the transition temperature region, the Hall resistance slope changes. This is more prominent near the low field which suggested a strong contribution of AHE. Near the transition temperature, the magnetisation has little value as the sample started to show the ferromagnetic behaviour. Thus it is possible that the magnetisation has some contribution in the Hall resistance slope which needs to be corrected. Hence the magnetisation model has been applied in this case.

But for GdN_HC film the high field slope at $5 \mathrm{~K}$ is much steeper than the $300 \mathrm{~K}$ slope which suggests that the carrier concentration is changing because as discussed above in these regions the AHE does not vary with field enough to noticeably change the slope. Technically we could use the same model for the GdN_HC film but it will not explain why the $5 \mathrm{~K}$ slope differs from the $300 \mathrm{~K}$ slope.

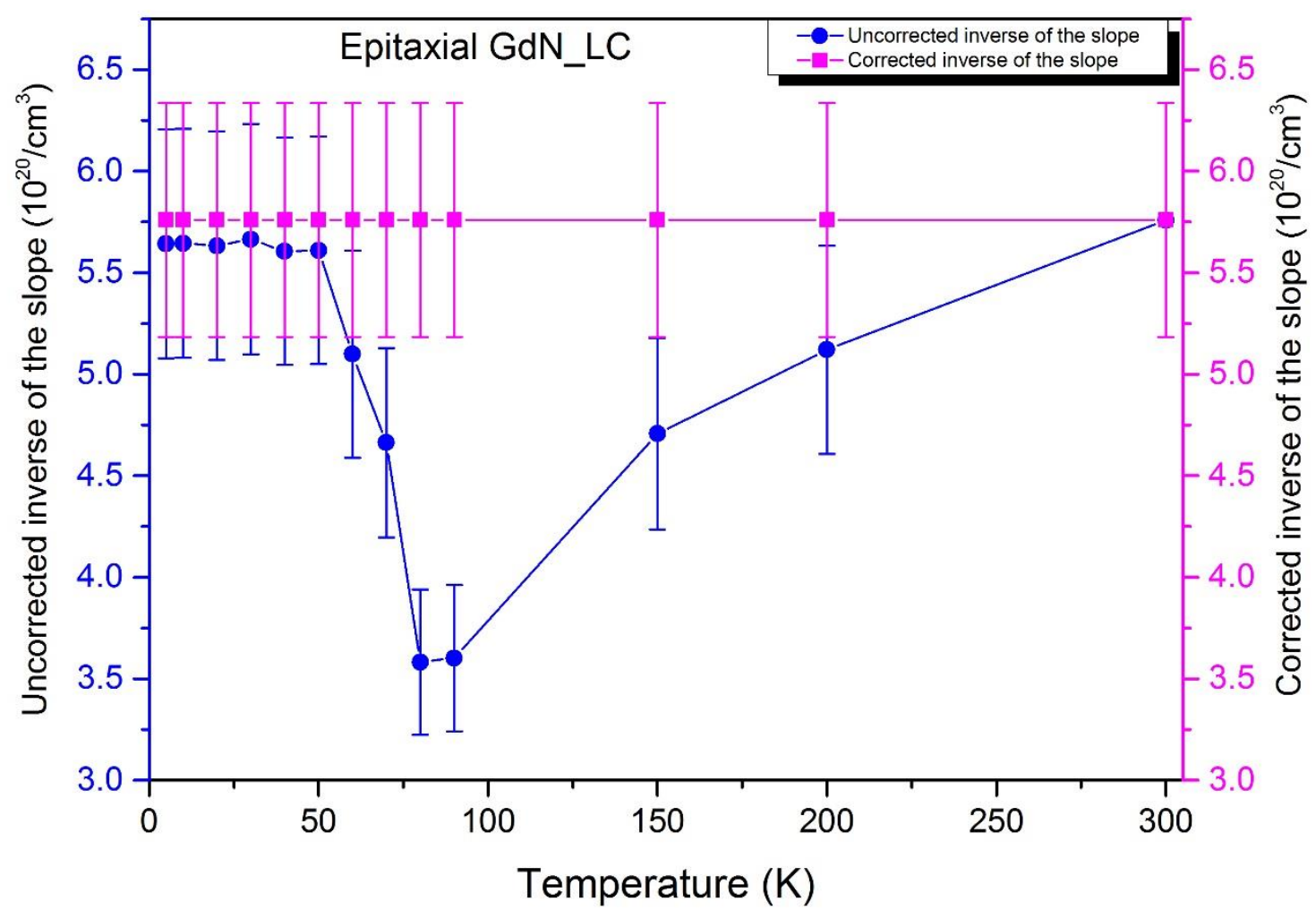

Figure 6.11: The uncorrected and corrected inverse of the slope as a function of temperature for the epitaxial GdN_LC film. 


\subsubsection{Interpretation of Hall effect results}

We have seen the carrier concentration implied by the slope is confused by a contribution from the anomalous Hall effect in the paramagnetic region. The uncorrected carrier concentration (i.e. inverse of the slope, experimental result without using our model) and corrected carrier concentration (after fitting with magnetisation data) obtained from the high field measurements as a function of temperature are shown in Figure 6.11. It is clear that the uncorrected carrier concentration is more dependent on temperature and its magnitude reduces as the temperature decreases from $300 \mathrm{~K}$ down to $70 \mathrm{~K}$ and then increases steeply until the temperature reaches $50 \mathrm{~K}$. Below $50 \mathrm{~K}$, the uncorrected carrier concentration remains fairly constant. This trend raised the question of whether the carrier concentration was really temperature dependent or if it appeared in such a way due to the magnetic contribution affecting the behaviour as we already discussed in the previous section. The data agree with a carrier concentration that is constant across the entire temperature range. The corrected carrier concentration is plotted in Figure 6.11 by a pink line and error bars.

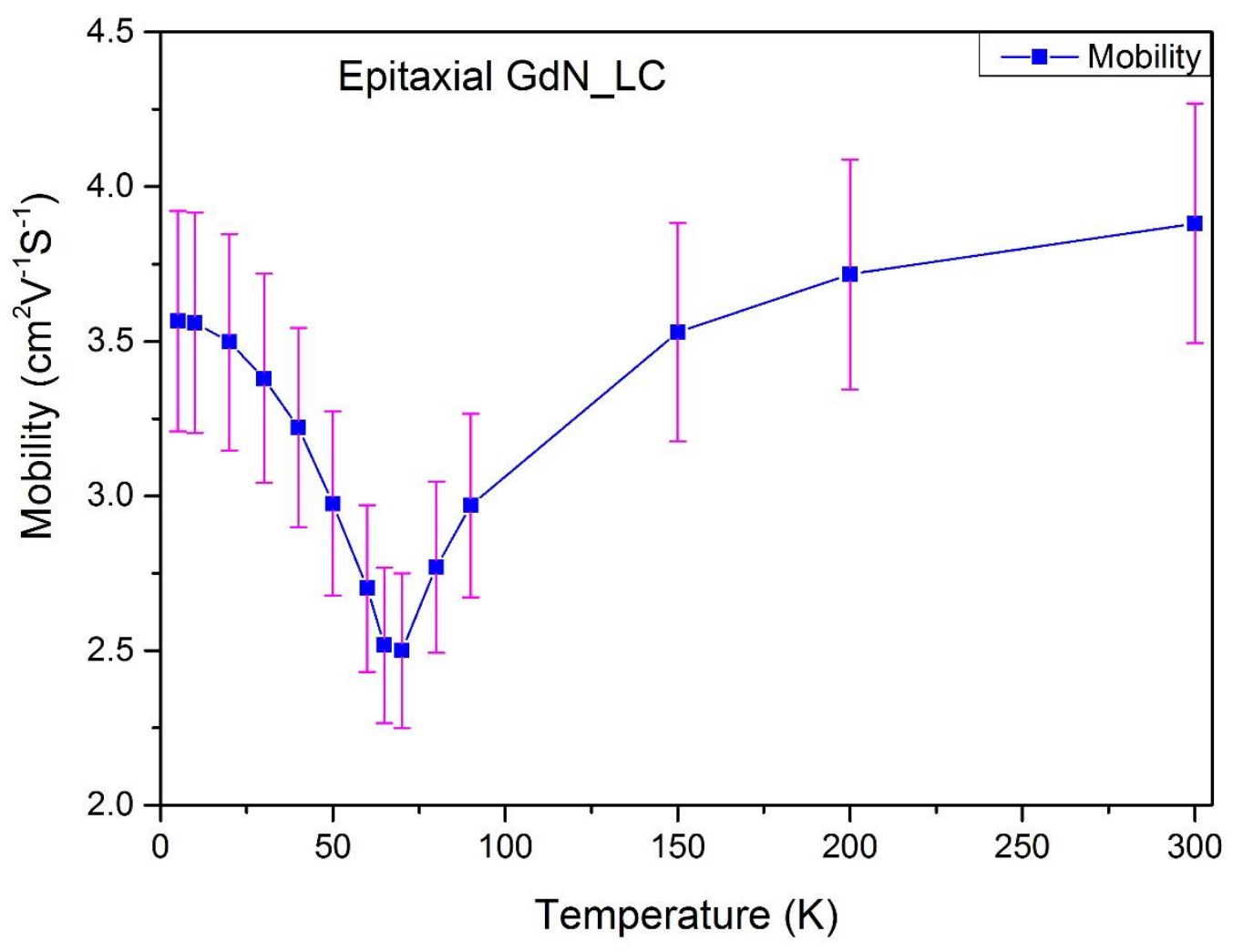

Figure 6.12: Mobility as a function of temperature of the epitaxial GdN_LC film. 
Figure 6.12 shows mobility as a function of temperature within the constant carrier concentration scenario. At the lowest temperature the mobility is obtained $\sim 3.5 \mathrm{~cm}^{2} \mathrm{~V}^{-1} \mathrm{~S}^{-1}$ which is around five times lower than the high carrier concentration film. We do not have any good explanation for why the mobility is much larger in the more heavily doped film. It could be possible that in the present case the free carriers scatter much more with the occupied state whereas in the other case carriers mostly scatter with the empty impurity state. As a result the velocity of the electrons reduces while they encounter the relatively heavier occupied state than the lighter empty state. Most notably, the mobility result at the transition temperature clearly suggests that the resistance anomaly is a signature of spin-disorder scattering while the carrier concentration is found to be constant at all temperatures.

The carrier concentration of this film at RT is of the order of $5.7 \pm 0.5 \times 10^{20} / \mathrm{cm}^{3}$ which suggest that insufficient free carriers are available to overfill the majority band and conductivity is dominated by the single-band carriers [53]. When compared with the epitaxial GdN_HC film, the higher resistivity of this film is attributed to its lower carrier concentration, which is associated with a relatively lower level of doping. The high carrier concentration indicates that the Fermi level lies above the bottom of the conduction band and the typical thermal activation model is simply not possible for this type of moderately high carrier concentration film.

The Hall effect result clearly indicates that the temperature dependent response of the uncorrected inverse of the slope is influenced by the magnetisation of the film in both paramagnetic and ferromagnetic regions. We conclude that the carrier concentration of this film is most likely constant and does not change with the temperature. 


\subsection{Summary and conclusion}

In this chapter we have presented the magnetic and transport results of a low carrier concentration epitaxial GdN film. The magnetic measurement was performed between $5 \mathrm{~K}$ to $300 \mathrm{~K}$ temperature regions in the in-plane direction. The magnetic results show that the transition temperature and saturation magnetisation are $64.5 \pm 1 \mathrm{~K}$ and $7 \pm 0.5 \mu_{\mathrm{B}} / \mathrm{Gd}^{3+}$, respectively. The experimental results show that near the transition temperature the magnetic fluctuation significantly contributes to the magnetic disorder scattering.

The resistivity, magnetoresistance and Hall effect have been measured from $5 \mathrm{~K}$ to $300 \mathrm{~K}$ temperature region. The higher resistivity of this sample as compared with the other epitaxial GdN_HC film is associated with the lower carrier density as obtained from the Hall effect measurement. Scattering from strong magnetic fluctuation has been observed near the transition temperature region which is reduced in the presence of a strong magnetic field. The resistance anomaly at $T_{c}$ is a signature of spin-disorder scattering which is supported by the mobility results. The magnetoresistance results show the maximum reduction of resistivity near the transition temperature region in the presence of highest magnetic field.

The initial Hall effect treatments found that the inverse of the slope is strongly temperature dependent which is questionable. Our interpretation based on the Brillouin function model in the paramagnetic region and SQUID results in the ferromagnetic region reveals that the Hall effect slopes are strongly influenced by magnetisation of the film. This is absolutely a new finding in the field of rare-earth nitride research and so far it has not been identified or reported by any other research group in this field.

The corrected Hall effect slopes indicate that the carrier concentration was shown to be independent of temperature for this epitaxial film which is a typical characteristic of a metallic film. Its metallic nature is further implied by the high carrier concentration which is obtained from the inverse of the slope is around $5.7 \pm 0.5 \times 10^{20} / \mathrm{cm}^{3}$ and the conductivity is only dominated by the majority-spin band. The result of this film is very interesting as one would see that the lower carrier concentration sample (GdN_LC) does not show temperature dependence whereas the higher carrier concentration sample (GdN_HC) did. This is very much the opposite of what we commonly see in the semiconductor. In conclusion, this GdN film is a moderately doped semiconductor with a metallic characteristic. 


\section{Chapter 7}

\section{Magnetotransport properties of a polycrystalline gadolinium nitride film}

\subsection{Introduction}

In this chapter we will present the magnetic and transport results of a polycrystalline GdN film. We would like to make it clear that the primary attention of this chapter is to understand the transport properties of this polycrystalline $\mathrm{GdN}$ film within the existing band structure scenario. In the previous two chapters we have discussed the results of higher carrier concentration and lower carrier concentration epitaxial films grown at elevated temperature. In both cases we have seen that the Fermi level lies well above the minimum of the conduction band and conductivity is most readily interpreted by the majority band. In the present case the situation is a bit different. This film has lower carrier concentration than the two epitaxial GdN films and shows the evidence of thermally activated behaviour at low temperature.

This chapter has three main Sections, i.e. 7.2, 7.3 and 7.4 whereas Section 7.3 comprised with two subsections, i.e. 7.3.1 and 7.3.2, respectively. In the first Section 7.2, the results of magnetisation measurements as a function of temperature and field are presented followed by the interpretation of the results in a separate subsection 7.2.1. The magnetisation measurement was initiated in order to understand the conduction process more systematically as in previous GdN films. Note that here we have compared the different transition temperatures of all GdN films presented in this thesis and the apparent reason for it has been discussed based on the magnetic polarons scenario. 
Section 7.3 begins with presenting the resistivity result as a function of temperature followed by an interpretation of this result discussed under a subsection 7.3.1. We have encountered difficulties to carry out the temperature dependent Hall effect measurement for this film and thus only room temperature Hall effect measurement was performed. The second subsection 7.3.2 contains the magnetoresistance results of this film. The magnetoresistance measurements were mainly performed in the ferromagnetic regions to see how the conductivity of the film is influenced in the presence of magnetic fields. The thermopower measurement was not performed on this film as the design of the thermopower rig was not completed by that time. The last Section 7.4 summarised all magnetic and transport results of this film.

Unlike the previous two GdN films, this film was grown at the Victoria University of Wellington, New Zealand, using a UHV system in the Molecular Beam Epitaxy Lab. The $135 \pm 15 \mathrm{~nm}$ polycrystalline film was deposited at room temperature onto a sapphire substrate by thermal evaporation of high purity Gd metal in the presence of a partial nitrogen pressure of $9.5 \times 10^{-5}$ Torr. To avoid oxidation on exposure to air, the film was capped with a protective layer of $50 \mathrm{~nm}$ of $\mathrm{GaN}$. 


\subsection{Magnetic results of the polycrystalline GdN film}

Magnetisation measurements of this polycrystalline $\mathrm{GdN}$ film were carried out from $2 \mathrm{~K}$ to 300 K using a Quantum Design SQUID magnetometer with the field oriented in-plane direction at the Robinson Research Institute, Gracefield.

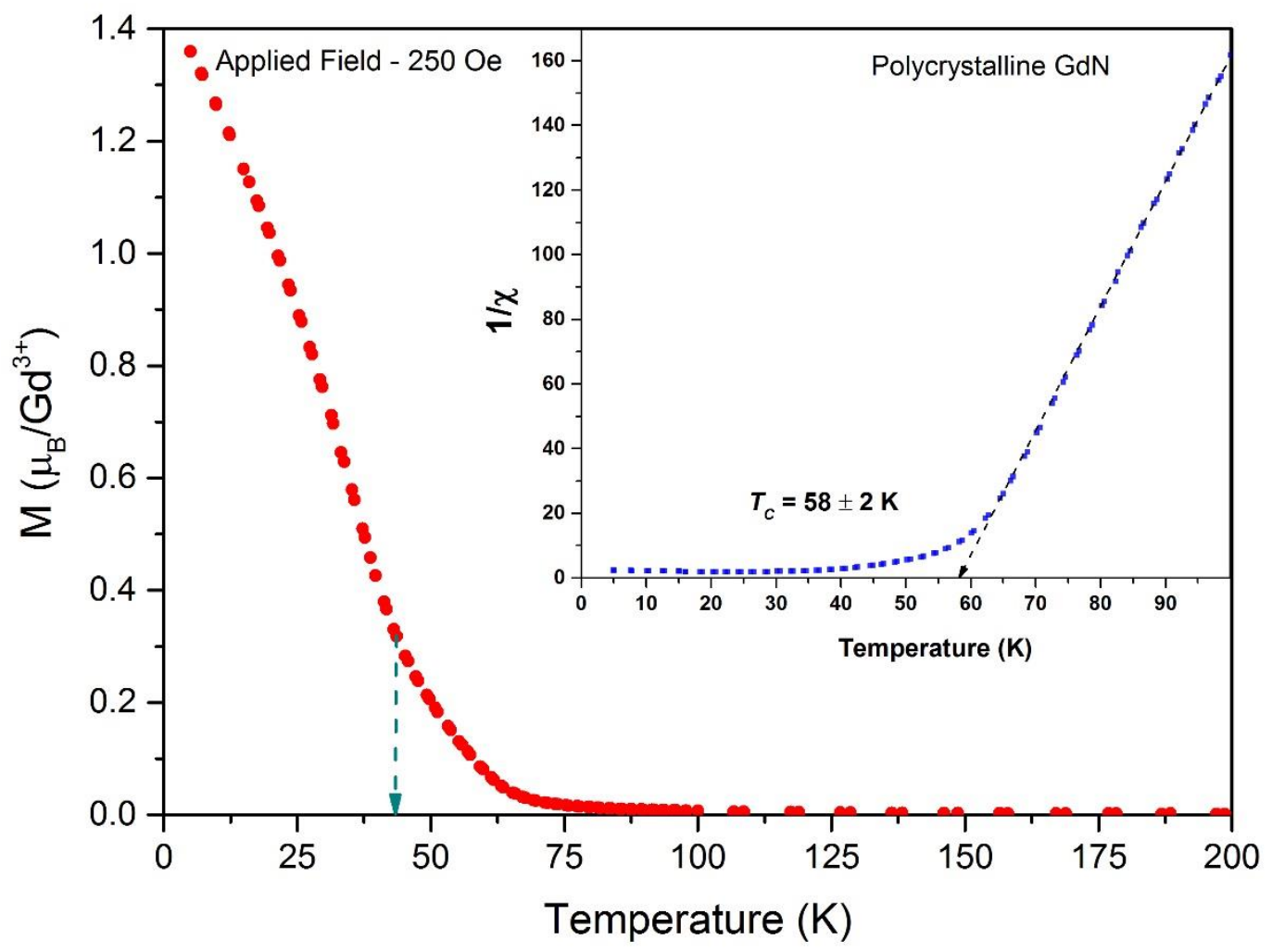

Figure 7.1: Magnetisation curve and Curie-Weiss fitting (inset) of the polycrystalline GdN film as a function of temperature.

Figure 7.1 shows the magnetisation as a function of temperature. The experiment was performed in the presence of 250 Oe magnetic field. At low temperature, below $60 \mathrm{~K}$, the polycrystalline GdN film shows an upturning characteristic, indicating the qualitative features of the ferromagnetic Curie temperature is in this region. To further obtain the transition temperature, Curie - Weiss fitting was performed as shown in the inset. The Curie - Weiss fit shows a ferromagnetic transition temperature is closer to $58 \pm 2 \mathrm{~K}$ which is lower than the commonly reported value of $70 \mathrm{~K}$ and also lower than the previous two epitaxial GdN films reported in Chapter 5 (Section 5.2) and 6 (Section 6.2), respectively. 
Interestingly, the polycrystalline GdN film shows a clear curvature in the magnetisation at low temperature, around $45 \mathrm{~K}$ coinciding with the double transition temperature of this film as marked by a little arrow. This feature we have seen in our epitaxial films presented in this thesis and also in previous research on epitaxial GdN film [97] but it was not identified in any polycrystalline GdN films by earlier researchers [44, 105]. This feature clearly suggests that although the transition temperature of $\mathrm{GdN}$ film is most widely recognised at $70 \mathrm{~K}$, the intrinsic transition temperature may be much lower, somewhere between $40 \mathrm{~K}$ to $50 \mathrm{~K}$.

Field dependent magnetisation measurements were performed at $5 \mathrm{~K}$ to obtain the saturation magnetisation of the film. The external magnetic field was applied along the film's surface. At higher fields, $4 f$ spins tend to align along the applied field, leading to the saturation magnetisation of the GdN film. Figure 7.2 shows the magnetisation as a function of field at 5 $\mathrm{K}$. The saturation magnetisation for the polycrystalline film is $5.3 \pm 0.7 \mu_{\mathrm{B}} / \mathrm{Gd}^{3+}$, significantly less than its commonly reported value of saturation moment $7 \mu_{\mathrm{B}} / \mathrm{Gd}^{3+}$.

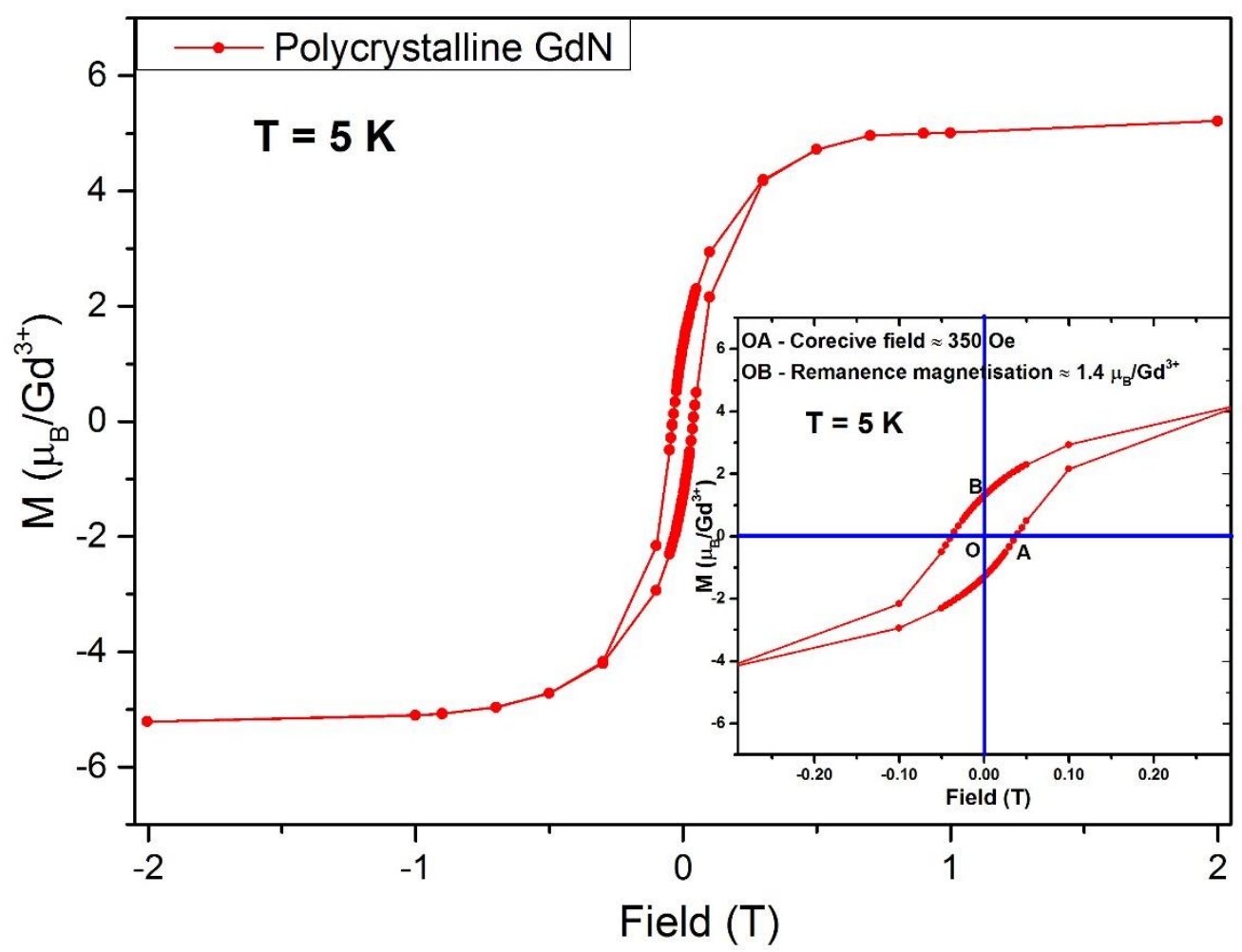

Figure 7.2: Magnetisation data of the polycrystalline $\mathrm{GdN}$ film as a function of field at $5 \mathrm{~K}$. Inset in the bottom-right panel shows the hysteresis loop at $5 \mathrm{~K}$. 
There are several factors which generally alter the saturation magnetisation of the film. Firstly, in order to determine the magnetisation in Bohr magnetons per gadolinium ion $\left(\mu_{\mathrm{B}} / \mathrm{Gd}^{3+}\right)$, the volume of the film must be known, which carries a significant uncertainty in this experiment. As we cannot measure the thickness of the film in-situ, we have considered using the RBS measurements. The area of the film was calculated manually using a ruler and optical microscope, which again introduced an additional uncertainty in the result. Therefore, we would like to acknowledge that the primary source of uncertainty comes from the thickness and the area of the film which is already included in the uncertainty result. In the present case, the saturation magnetisation is around $40 \%$ lower than the commonly reported value of $7 \mu_{\mathrm{B}} / \mathrm{Gd}^{3+}$. This signals that except for the uncertainty there must be a systematic error for showing such a low saturation magnetisation which we discuss later in the interpretation Section 7.2.1.

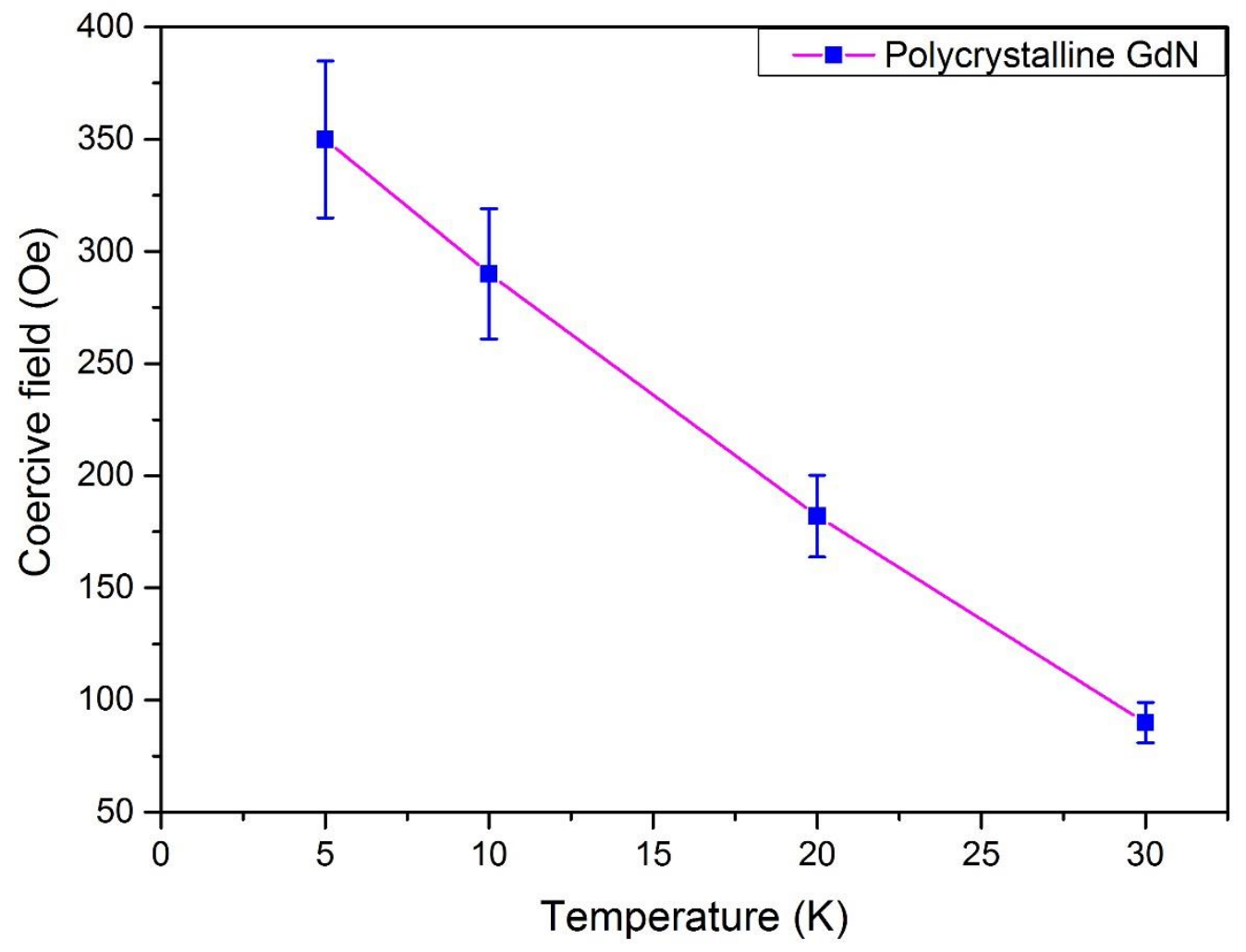

Figure 7.3: Coercive field of the polycrystalline $\mathrm{GdN}$ film as a function of temperature. 
Hysteresis curves provide further evidence about the ferromagnetic state of GdN. A coercive field of $350 \mathrm{Oe}$ and remanence of $1.4 \mu_{\mathrm{B}} / \mathrm{Gd}^{3+}$ were extracted from the hysteresis curve at $5 \mathrm{~K}$, shown clearly in inset Figure 7.2, where the value of OA and OB define the coercive field and remanence magnetisation of the film, respectively. Figure 7.3 shows the coercive fields obtained at different temperatures. As the temperature decreases the magnitude of the coercive field is increased as is commonly found. We expect the coercivity to change with temperature due to change in magnetic anisotropy energy.

\subsubsection{Interpretation of magnetic results}

In this section we will discuss the apparent reason for the lower transition temperature and lower saturation magnetisation of this film and compare results with our previous two epitaxial films. There is a wide number of reports quoting different values of the Curie temperature for GdN films and several interpretations have been proposed to explain this variation. Interestingly, the significant reason which causes the variation of transition temperature has not taken the primary attention earlier, until recently our research group had proposed the magnetic polaron concept which works nicely on epitaxial films. Therefore, it would be worth considering the magnetic polaron scenario which is developed based on the nitrogen vacancy concept and can be utilised in the present case.

This variation of transition temperatures of all GdN films presented in this thesis is directly related to the nitrogen vacancy. A previous study of epitaxial GdN films [97, 177] showed an interplay between magnetism and the density of carriers. It was reported that the nitrogen vacancies promote the formation of magnetic polarons in GdN. Note that the previous study was only focused on the epitaxial GdN films where all films were grown at the elevated temperature. The present author had an opportunity to carry out the magnetic measurement of one of the GdN films whose results have been highlighted in the published article [97]. It is worth seeing how effectively this model can be utilised for explaining the lower transition temperature of the present polycrystalline GdN film here. In the following paragraphs we will see that this model also works nicely for the low nitrogen vacancy polycrystalline GdN film. 
Magnetic polaron picture for $T_{c}$

In Chapter 1, we already have discussed that the source of electrons in GdN films are the nitrogen vacancy $\left(V_{N}\right)$. Punya et al. performed theoretical calculations and speculate that the $V_{N}$ can provide up to three electrons out of which two are closely bound to $V_{N}$ in a singlet state [173]. Only the third electron binds very weakly to the $V_{N}$, which acts as a free carrier in the conduction band at high temperatures. These free carriers are the main source of the conductivity of GdN films. If the temperature decreases, then the carrier from $V_{N}$ starts to freeze out from the conduction band and occupy the third $V_{N}$ level, resulting in a large electron cloud or magnetic orbit forming around the $V_{N}$. Similarly, the neighbouring $\mathrm{Gd}^{3+}$ ions can also have their exchange mediated within the electron cloud. Therefore, we have seen a magnetic polaron $[97,178]$.
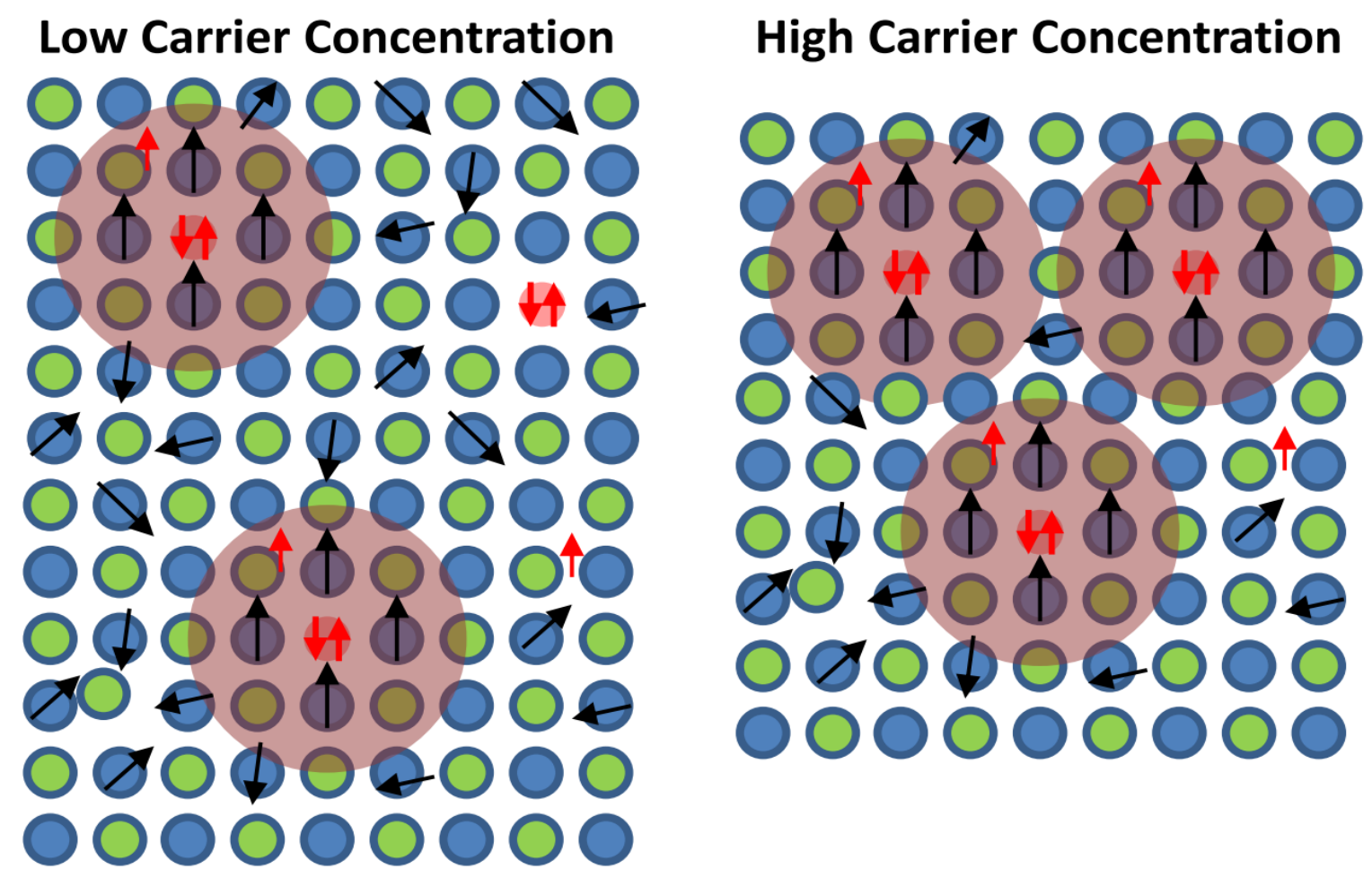

Figure 7.4: A diagram of the magnetic ordering by polarons, for low and high carrier concentration GdN films. 
To visualise the idea, we have presented the schematic illustration of the polaron scenario in Figure 7.4. The $\mathrm{Gd}^{3+}$ and $\mathrm{N}^{3-}$ ions are shown by blue and green circles, respectively, and the small red circles represent nitrogen vacancies $\left(V_{N}\right)$. The black arrows show the spins of $\mathrm{Gd}^{3+}$ and the red arrows are the spins of the electrons donated by $V_{N}$. Magnetic polarons are believed to form near the transition temperature which is around $65 \mathrm{~K}$ for our epitaxial films. The formation of polarons are indicated by the large brown circle around $V_{N}$ in Figure 7.4. Once the sample temperature reaches to its transition temperature, the spins of $\mathrm{Gd}^{3+}$ ions are well ordered only near $V_{N}$ but outside the electron clouds, the spins of $\mathrm{Gd}^{3+}$ ions are still randomly orientated. As a result the ferromagnetic behaviour is seen inside the electron cloud whereas paramagnetic nature is prominent outside the electron cloud. For higher carrier concentration films, i.e. high $V_{N}$ density, the polaron density is also high enough such that more electron clouds are formed and ultimately they start to overlap with each other. Therefore, once all electron clouds are merged with each other the polarons coalesce, resulting that most of $\mathrm{Gd}^{3+}$ spins are well ordered and exhibits a uniform ferromagnetic phase. In contrast, for a lower carrier concentration film like the present polycrystalline $\mathrm{GdN}$ film, the polaron density is also low. The transition temperature of this film is found to be lower than both epitaxial films, suggesting that here the electron clouds do not overlap with each other around $65 \mathrm{~K}$. Once the temperature reaches near its transition temperature $(\sim 60 \mathrm{~K})$, the polarons nucleate, where we expect only the spins of $\mathrm{Gd}^{3+}$ ions near $V_{N}$ will order in a systematic way. The rest of the $\mathrm{Gd}^{3+}$ ions in the film will not contribute to ferromagnetism until the intrinsic critical temperature of $\mathrm{Gd}$ is reached which is believed between $40 \mathrm{~K}$ to $50 \mathrm{~K}$.

Based on the polaron model, we have explained the ferromagnetic behaviour observed for all epitaxial and polycrystalline GdN films. The epitaxial GdN_HC and GdN_LC films show a uniform magnetic transition near $67 \mathrm{~K}$ and $65 \mathrm{~K}$ respectively, indicating that the nitrogen vacancy concentration is sufficient for the polarons to overlap and all the $\mathrm{Gd}^{3+}$ spins to order when polarons nucleate. Thus, we see the strongest ferromagnetic response near $67 \mathrm{~K}$ and 65 K for epitaxial GdN_HC and GdN_LC films. The polycrystalline GdN film shows a relatively weak onset of ferromagnetism near this temperature, followed by a stronger magnetic transition below $50 \mathrm{~K}$. Therefore, we conclude that the formation of polarons strongly influences the transition temperature of all GdN films presented in this thesis. 


\section{Lower saturation magnetisation}

We have speculated a few possible reasons for the lower saturation magnetization observed in this film. First is related to film's thickness which is obtained from the RBS experiment and accurate within $10 \%$ uncertainty. After growing the film, we cut it into a few separate pieces for carrying out several measurements. It is possible that the sample that went to the SQUID for the magnetic measurement was a different thickness than the piece used for the thickness measurement (RBS experiment). This introduces another uncertainty in the measurement which however is not included in the uncertainty estimated earlier because we have no way to estimate how much this might be, but it is unlikely to come near to the $40 \%$ that might explain the decrement we observe.

Secondly, it is possible that some Gd ions do not order magnetically. We are uncertain whether there are any other phases that exists in the sample, but it is possible that grain boundaries do not order magnetically in the way the bulk of material does. As a result, the ferromagnetic interaction fails at the grain boundaries and exhibits the lower saturation magnetisation of the film.

Finally, we note that it is unclear whether the polaron scenario of the previous section might explain the lower saturation magnetisation in films with low nitrogen vacancy concentration. 


\subsection{Transport results of the polycrystalline GdN film}

\subsubsection{Resistivity result}

This section reports the temperature dependent resistivity and magnetoresistance results of the polycrystalline GdN film for which the room temperature Hall effect measurement gave the carrier concentration of $(3 \pm 0.5) \times 10^{19} \mathrm{~cm}^{-3}$ and it leads to the mobility of the film which is around $6 \mathrm{~cm}^{2} / \mathrm{V}$. sec.

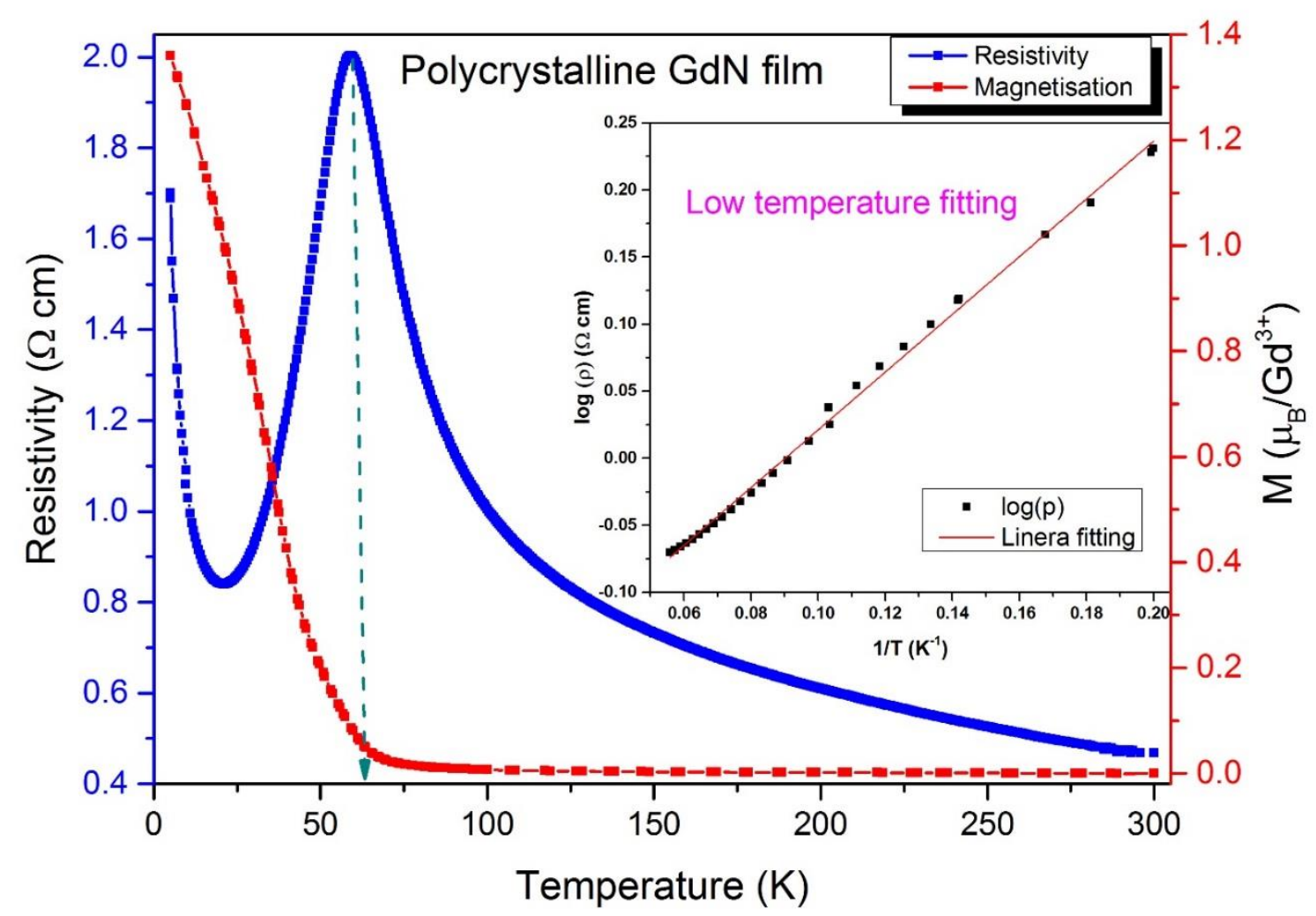

Figure 7.5: Temperature dependent resistivity and magnetisation of the polycrystalline GdN film.

Inset: Arrhenius plot at low temperature. 
The corresponding temperature dependent resistivity and magnetisation are shown in Figure 7.5. The resistivity experiment was performed between $300 \mathrm{~K}$ to $5 \mathrm{~K}$. The magnetisation data has been shown again in the same figure for purpose of clarity to see how well the resistivity peak $(\sim 59 \mathrm{~K})$ matches with the transition temperature $(58 \pm 2 \mathrm{~K})$. The resistivity graph shows a similar shape to the epitaxial GdN films although a substantially larger resistivity with more pronounced temperature dependence is seen in this case. The figure shows, in the ferromagnetic phase the anomaly becomes stronger, with the resistivity dropping by up to a factor of two below $T_{c}$. In contrast to the epitaxial films, in this film there is a sudden rise in resistance at low temperatures which we interpret in terms of Arrhenius excitation. Approximately below $15 \mathrm{~K}$, the activation energy is estimated using the Arrhenius equation is about $0.18 \mathrm{meV}$ and shown in the inset Figure 7.5.

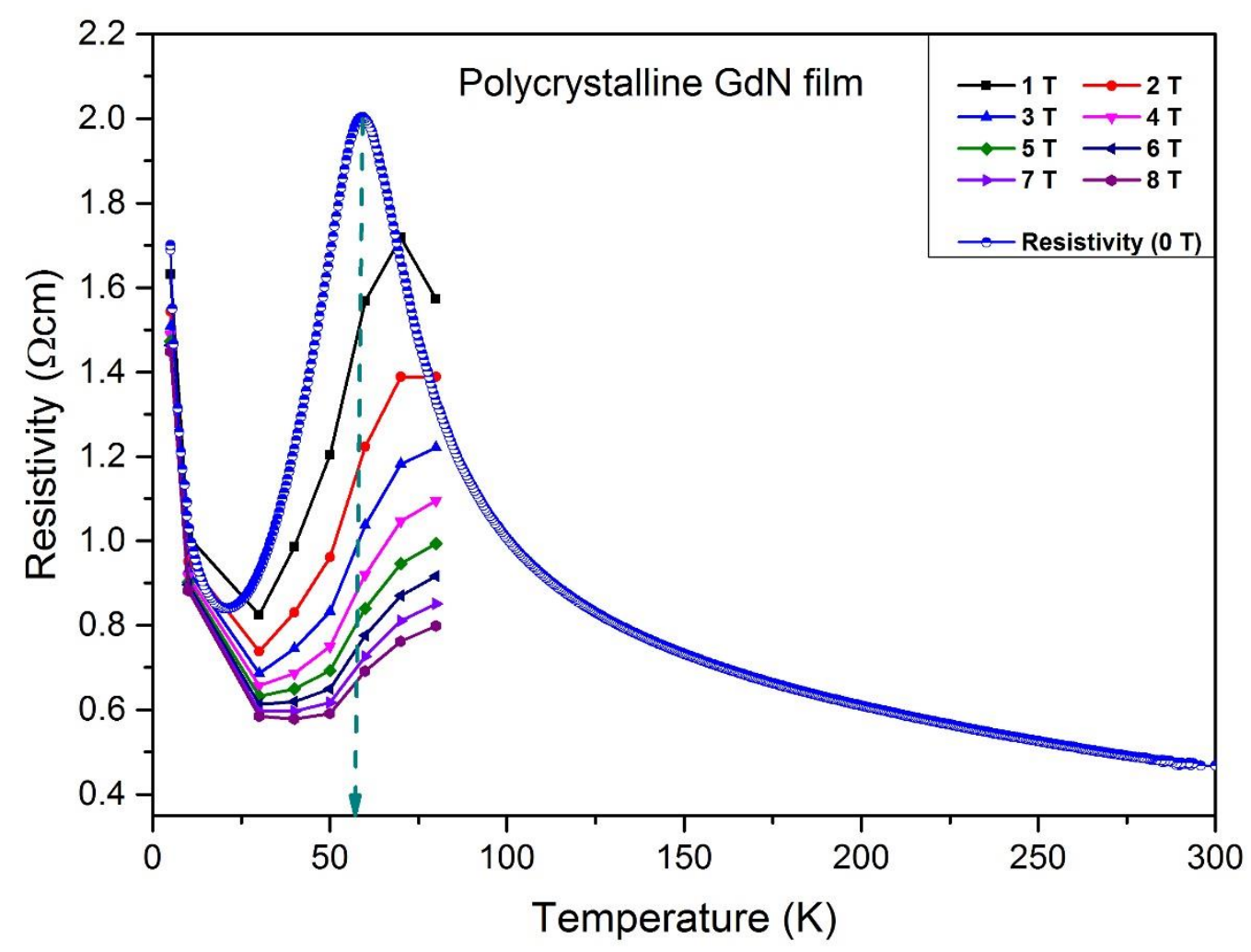

Figure 7.6: Resistivity of the polycrystalline GdN film at different fields as a function of temperature. 
The influence of magnetic fields in the resistivity measurement has also been seen in the present case. Figure 7.6 shows the resistance as a function of the strength of the magnetic field. The resistivity reduces significantly in the presence of the highest magnetic field (8T), as expected because the field suppresses magnetic disorder scattering. The magnetic field dependence of the resistivity result apparently disappears below $15 \mathrm{~K}$. This experimental result clearly indicates that the whole resistivity peak is associated with magnetic scattering, in disagreement with the interpretation of it being associated with a change in the carrier concentration used by Granville et al. [44]. The fuller description of the field dependency resistivity result has been examined thoroughly and reported in Section 7.3.2.

\subsubsection{Interpretation of resistivity results}

This film is found to be much more resistive than both of the epitaxial films. Resistivity is affected by the carrier concentration and the scattering; remarkably in this case we can separate them. In earlier Chapters, 5 (Epitaxial GdN_HC) and 6 (Epitaxial GdN _LC), we have seen that the resistivity peak occurs due to the magnetic disorder scattering [53] and this correlation we have also seen in the present case. The low mobility $\left(6 \mathrm{~cm}^{2} / \mathrm{V} . \mathrm{sec}\right)$ of the carriers in the present case estimated at RT indicates the carriers experience more frequent scattering and reduces the conductivity of the film.

The resistivity of this sample cannot be simply explained as in our previous two epitaxial films as it has shown two contrasting phenomena just above and below $15 \mathrm{~K}$. Hence, the situation is unique for this small carrier concentration film as discussed later. Below $15 \mathrm{~K}$, the resistivity rises gradually (Figure 7.5) with the reduction of temperature and suggested its semiconducting character in the ground state. In contrast, both epitaxial films discussed earlier (Chapter 5 and Chapter 6) show little evidence of such an upturn below $15 \mathrm{~K}$.

To tackle this anomaly we interpret the results based on the experimental data. Figure 7.6 does not show any significant changes of resistance below $15 \mathrm{~K}$ in the presence of the magnetic field. As expected for low temperature, no magnetic disorder scattering is observed, and the resistivity result does not show any evidence of the magnetic field dependence. This is because of that impurity states are localised just below the conduction band and one electron is not fully unbound in these states which then adds $0.18 \mathrm{meV}$ energy. If there are more nitrogen vacancies, 
then this free electron comes into the conduction band as we have seen in the epitaxial films where the nitrogen vacancy concentration is so high that all unbound electrons are found to be in the conduction band. Thus in this case we believe we reduced the carrier concentration by reducing the number of nitrogen vacancies and presumably they are no longer overlapping below $15 \mathrm{~K}$, and we have thermally activated behaviour.

The situation is different when we consider behaviour above $15 \mathrm{~K}$. Even at the modest temperature of $100 \mathrm{~K}$, those impurity states or nitrogen vacancy states are completely ionised. When the carrier concentration gets above a certain number then polarons begin to overlap; as a result free electrons (from nitrogen vacancies) travel through the impurity band and they are no longer localised. This causes the conduction process through the impurity band rather than electron excited from impurity states to the conduction band. This is exactly what is happening in the present case. The temperature dependence of the resistivity peak only appears due to the scattering unless the band change at the transition temperature.

The resistivity results of this film suggest that the film grown at room temperature and having the carrier concentration lower than $10^{20} / \mathrm{cm}^{3}$; most likely has a small energy gap between the occupied localised states and the extended states below $15 \mathrm{~K}$, whereas the activation behaviour becomes irrelevant well above this temperature and the conduction process occurs through the impurity band. We now concluded that the polycrystalline sample is showing a metallic nature above $15 \mathrm{~K}$ and found to be semiconducting below this temperature. The estimated lower carrier density of this film suggests this film is a better representation of a weakly doped semiconductor. 


\subsubsection{Magnetoresistance (MR) results}

We have performed the magnetoresistance measurement on a polycrystalline GdN film as a function of temperature under two different magnetic field directions. The results show the highest reduction of the resistance as compared to any other GdN films reported in the literature so far $[24,44]$. We have focused on the magnetoresistance measurement between $5 \mathrm{~K}$ to $80 \mathrm{~K}$ temperature regions where magnetic fluctuations play a crucial role towards changing the resistance of the film.

Figures 7.7 and 7.8 illustrate the magnetoresistance of the polycrystalline film measured at various temperatures for fields applied perpendicular and parallel to the film plane. The result shows the strong negative magnetoresistance near the transition temperature, similar to the epitaxial GdN_HC film presented in Chapter 5 (Section 5.3.2). The magnitude changes by about $70 \%$ at $T_{c}$ and drops to $15 \%$ at the lowest temperature of $5 \mathrm{~K}$. Such a huge change is noticeable and it worthwhile considering this material for a magnetic sensing device.

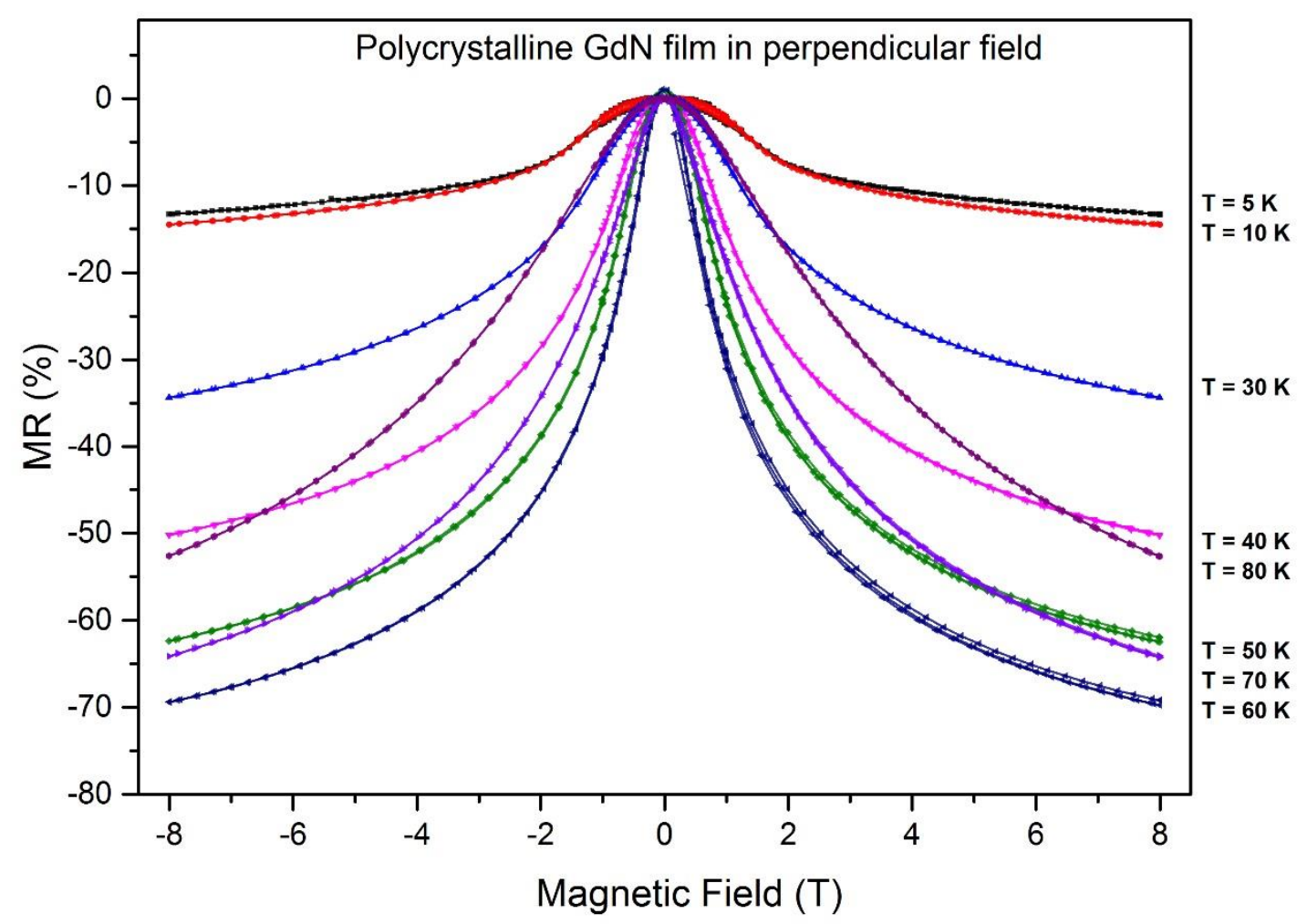

Figure 7.7: Magnetoresistance for the polycrystalline GdN film in applied fields perpendicular (outof-plane) to the film surface at different temperatures. 


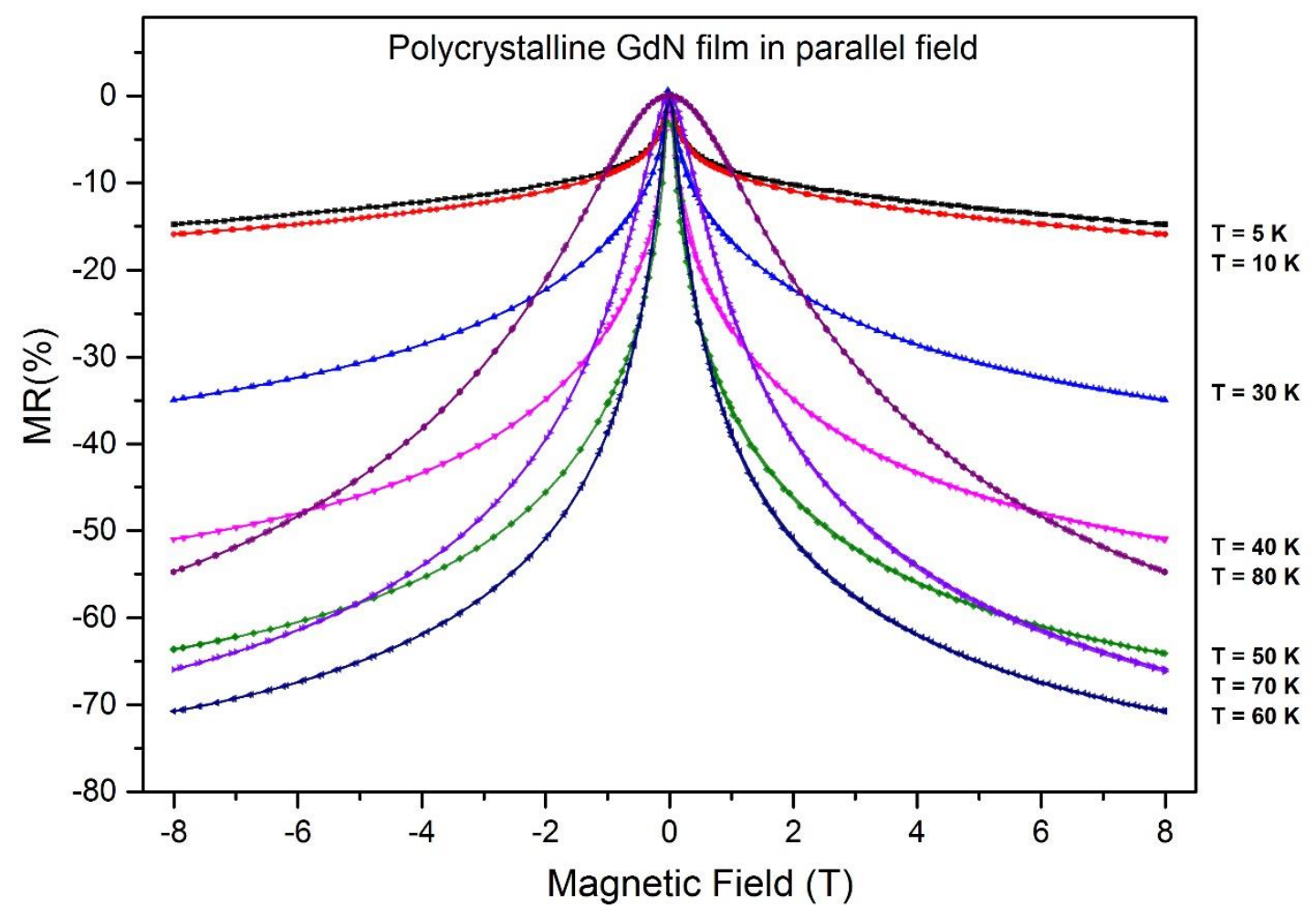

Figure 7.8: Magnetoresistance for the polycrystalline $\mathrm{GdN}$ film in applied fields parallel (in-plane) to the film surface at different temperatures.

The largest values observed near $T_{c}$, approaching negative $70 \%$ in a field of $\pm 8 \mathrm{~T}$ is the results from magnetic field suppression of the spin disorder scattering. This feature clearly signals that the mobility increases significantly as magnetic fluctuations reduce in the presence of a magnetic field. Figure 7.6, shown in the resistivity section also supports this evidence where we have seen the dominating contribution of scattering is reduced in the presence of a magnetic field. At high temperature, carriers are not well oriented and significantly experience scattering, which reduces the mean free path. The scattering contribution can be especially strong in low carrier concentration materials where even small wave-vector fluctuations are sufficient to reverse the carrier momentum [176]. Similar behaviour is expected for this GdN film which exhibits lower carrier concentration than the epitaxial GdN_HC film and also leads to negative magnetoresistance. 
At low temperatures (Figure 7.9) a cusp is seen for small parallel fields while a much broader maximum is observed in small perpendicular applied fields. This appears to be due to the shape anisotrophy as discussed a few times earlier which requires a minimum of $2.1 \mathrm{~T}$ magnetic field in the perpendicular direction to show the saturation magnetisation. In this case the two curves (black colour) for the magnetic field going up and down are merged together far below $2.1 \mathrm{~T}$ which is again noteworthy to mention and most likely relate with the films' saturation magnetisation as it is found to be lower than the commonly expected value of $7 \mu_{\mathrm{B}} / \mathrm{Gd}^{3+}$.

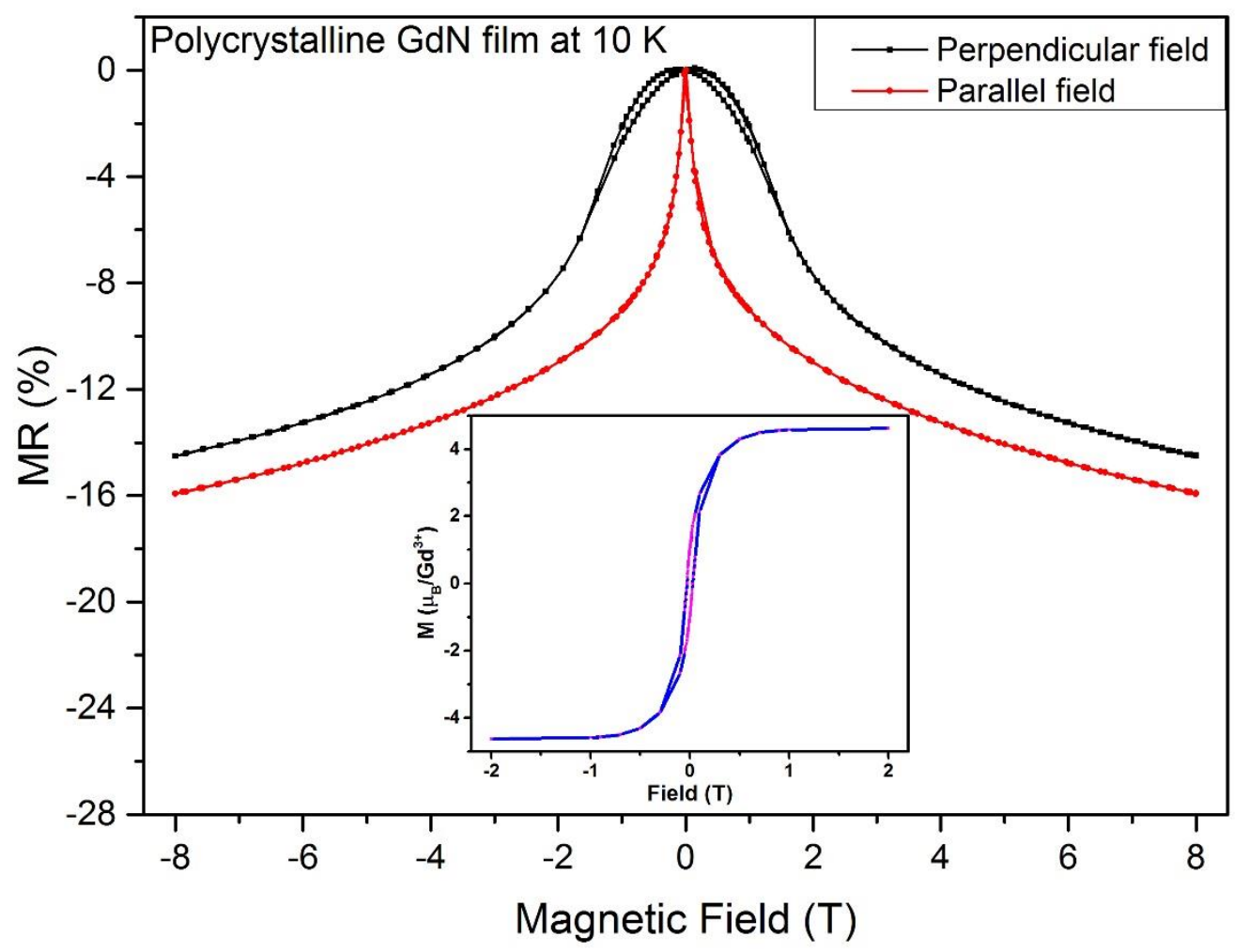

Figure 7.9: Magnetoresistance of the polycrystalline $\mathrm{GdN}$ film at $10 \mathrm{~K}$ measured with the applied magnetic field perpendicular (black line) and parallel (red line) to the film surface. The hysteresis loop under the perpendicular applied field has a coercive field $0.3 \mathrm{~T}$ while under the parallel applied field it is only about $0.03 \mathrm{~T}$. Inset: magnetisation as a function of applied magnetic field parallel to the film surface, where the coercive field estimated from the hysteresis loop is approximately $0.03 \mathrm{~T}$. 
Figure 7.9 presents the negative magnetoresistance of polycrystalline samples at $T=10 \mathrm{~K}$ under two orthogonal applied fields: a perpendicular field, and a parallel field to the applied current direction to the films' area. The hysteresis loop is observed when the field is perpendicular to films' surface area, for which the coercive field is obtained to be about $0.3 \mathrm{~T}$. This is much bigger than the in-plane coercive field of $0.03 \mathrm{~T}$. The inset shows the magnetisation as a function of the parallel applied field at $10 \mathrm{~K}$ for which the coercive field is obtained to be about $0.03 \mathrm{~T}$. The interpretation of hysteresis curve and the associated coercive field in MR results are difficult and needs further thought.

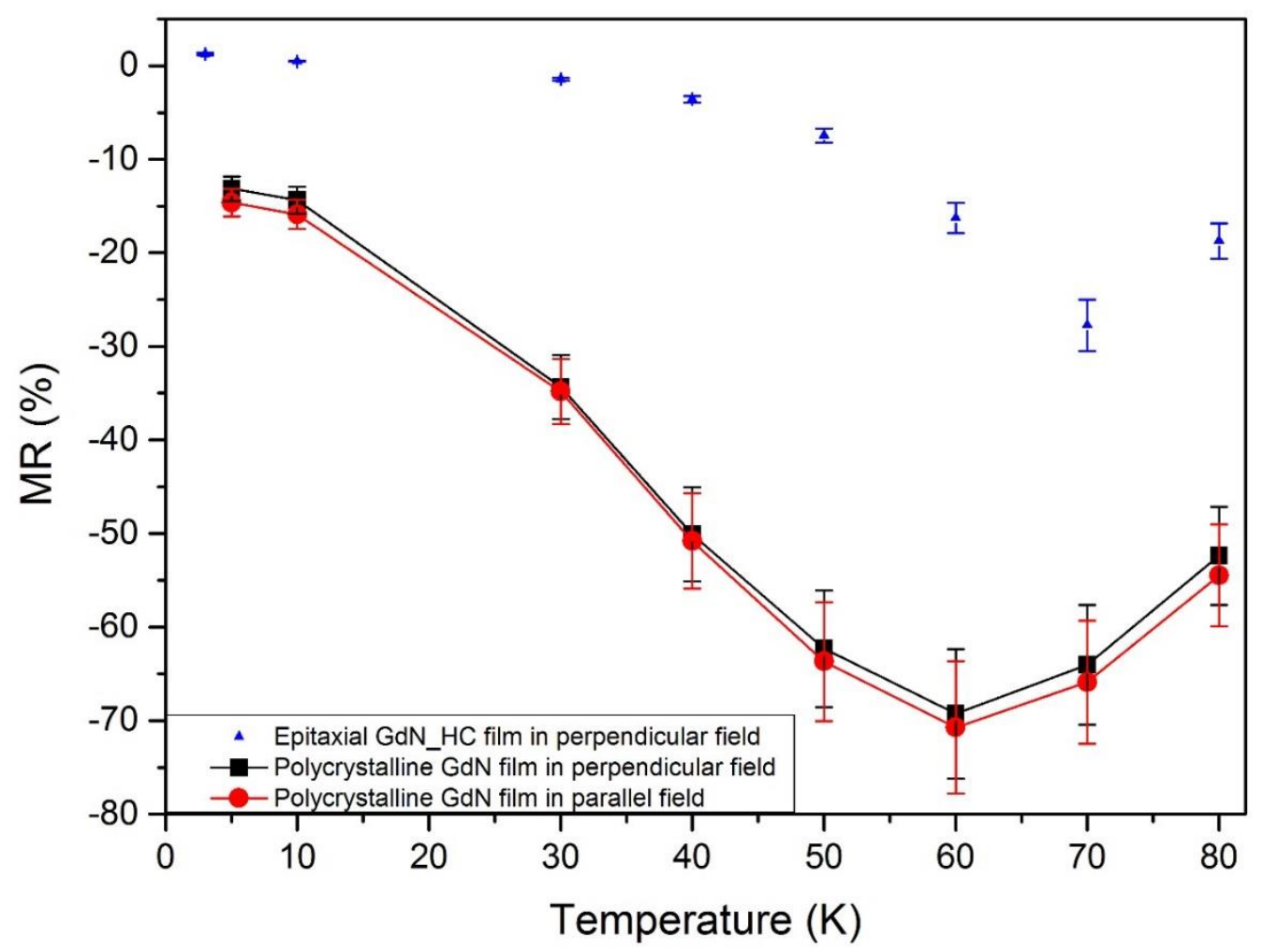

Figure 7.10: Comparison of high-field MR results of the epitaxial GdN_HC film in perpendicular field and the polycrystalline GdN film in perpendicular and parallel field orientations.

The magnetoresistance of polycrystalline $\mathrm{GdN}$ film has been performed at different temperatures and two different field orientations. The magnitude of MR is huge, but we have not seen any noticeable difference of MR in perpendicular and parallel field directions as shown in Figure 7.10. It would be nice to compare the magnitude of MR for both epitaxial GdN_HC and polycrystalline films. The magnitude of MR changes more dramatically for the polycrystalline film than the epitaxial film GdN_HC which shows the magnitude of MR larger 
than that of the epitaxial GdN_HC film as presented in the same Figure 7.10. As the polycrystalline film is more disordered than the epitaxial GdN_HC film, thus one would expect a significant dominating contribution from the grain boundaries which is suppressed in the presence of magnetic field.

Our experimental results clearly show that scattering plays a significant role in determining the magnitude of MR and it affects the relative contributions to the resistivity of GdN films; an innovative and one of the fundamental discoveries on this material.

\subsection{Summary and conclusion}

In this chapter we report the magnetic and transport properties of a polycrystalline GdN film grown at room temperature. The transition temperature of this film is obtained $58 \pm 2 \mathrm{~K}$, lower than the both epitaxial films and commonly reported value of $70 \mathrm{~K}$. The different value of $T_{c}$ is strongly influenced by the nitrogen vacancies or carrier concentration, which has been explained based on the existing magnetic polaron concept. Near the transition temperature the magnetic disorder scattering reduces which significantly influences the mobility and conductivity of the film. The saturation magnetisation of this film is found to be $5.1 \pm 0.7$ $\mu_{\mathrm{B}} / \mathrm{Gd}^{3+}$, less than its commonly reported value of saturation moment $7 \mu_{\mathrm{B}} / \mathrm{Gd}^{3+}$. The lower saturation magnetisation of the film is likely related to some of the Gd ions do not order ferromagnetically.

The polycrystalline GdN film is the most resistive among all GdN films discussed in this thesis. The Hall effect data shows that the carrier concentration at room temperature is about $(3 \pm 0.5$ $\left.\mathrm{x} 10^{19}\right) \mathrm{cm}^{-3}$ which is lower than both epitaxial films and insufficient to overfill the majority band. The lower carrier concentration which primarily arose from the low nitrogen vacancies, is one of the reasons showing the higher resistivity of this film as compared to other GdN films reported in previous Chapters 5 and 6. It is to be noted that the enhanced disorder scattering due to the polycrystalline structure of the film dominates the resistance of the film near the transition temperature region which is mostly suppressed under the magnetic field. The resistivity result below $15 \mathrm{~K}$ temperature has been interpreted based on the thermal activation model where we believe at least one electon is not fully unbound in the nitrogen vacancy state and adds $0.18 \mathrm{meV}$ energy to excite to the conduction band. The continuation of the thermal 
activation behaviour becomes flat once we get to the temperature well above the energy difference $(0.18 \mathrm{meV})$ between the donor state and the conduction band. In this circumstance polarons start to overlap and conduction process occurs through the impurity band. Within this scenario, above $15 \mathrm{~K}$ the film displays the typical characterestic of a metallic film as the carrier concentration is found to be approximately fixed. The two different behaviour of this film, i.e. below $15 \mathrm{~K}$ and above $15 \mathrm{~K}$ are quite unique and have not been identified and reported earlier.

The negative magnetoresistance of the polycrystalline film (Figures 7.7 and 7.8) is strongest around the transition temperature, similar to the epitaxial GdN_HC film. Interestingly, the magnitude of MR for the polycrystalline film is much bigger than the epitaxial GdN_HC film and it is around $70 \%$ in a field of $8 \mathrm{~T}$ at $60 \mathrm{~K}$ which is a quite large value and obtained for the first time on any REN material. Such an enhancement in the magnetoresistance is particularly noteworthy given that the resistivity of the polycrystalline film exceeds that of the epitaxial GdN_HC film by a factor of about 40. The experimental results suggest that the magnetic fluctuation reduces in the presence of an applied field which therefore minimise the scattering rate and enhances the conductivity of the film. 


\section{Chapter 8}

\section{Magnetotransport properties of samarium nitride films}

\subsection{Introduction}

Preliminary transport measurements were carried out to investigate the conducting nature of SmN film and this chapter will present the results. This chapter has two main sections. The first section reports the resistivity, temperature dependent Hall effect, magnetoresistance and thermopower results of the epitaxial $\mathrm{SmN}$ film. In addition, in the thermopower section we show the thermopower result of a polycrystalline $\mathrm{SmN}$ film and compare the result with the epitaxial one. We are unable to make the resistance measurement of polycrystalline SmN film because the film was reacted; however, that is not affecting the thermopower measurement as there was no current flowing through that high resistance.

For the purposes of the present study, the $90 \pm 10 \mathrm{~nm}$ thick epitaxial $\mathrm{SmN}$ film was grown at temperature $\left(400^{\circ} \mathrm{C}\right)$ by the molecular beam epitaxy (MBE) method using the nitrogen source by our collaborator in France. After the growth, the film was capped by $110 \pm 10 \mathrm{~nm} \mathrm{GaN}$ to prevent oxidation. This film was deposited on the YSZ substrate (100) as the thermal conductivity of this substrate is found to be very low which makes it an ideal candidate for the thermopower measurement [155].

The polycrystalline $\mathrm{SmN}$ film was grown at the thin film deposition lab in the Victoria University of Wellington using a vacuum chamber evacuated to a base pressure in the range of $10^{-8}$ mbar. This film was deposited on a glass substrate with pre-deposited Au contacts on top of the substrates. Sm metal was evaporated in the presence of an atmosphere of pure $\mathrm{N}_{2}$ gas at a pressure between $10^{-4}$ and $10^{-5}$ mbar. In contrast to the other SmN film, it was deposited at an ambient-temperature, exhibiting polycrystalline nature. To prevent oxidation, all ex-situ measurements were performed on a film protected by a $100 \mathrm{~nm}$ AIN capping layer. 


\subsection{Transport results of the epitaxial $\mathrm{SmN}$ film}

\subsubsection{Resistivity and Hall effect results}

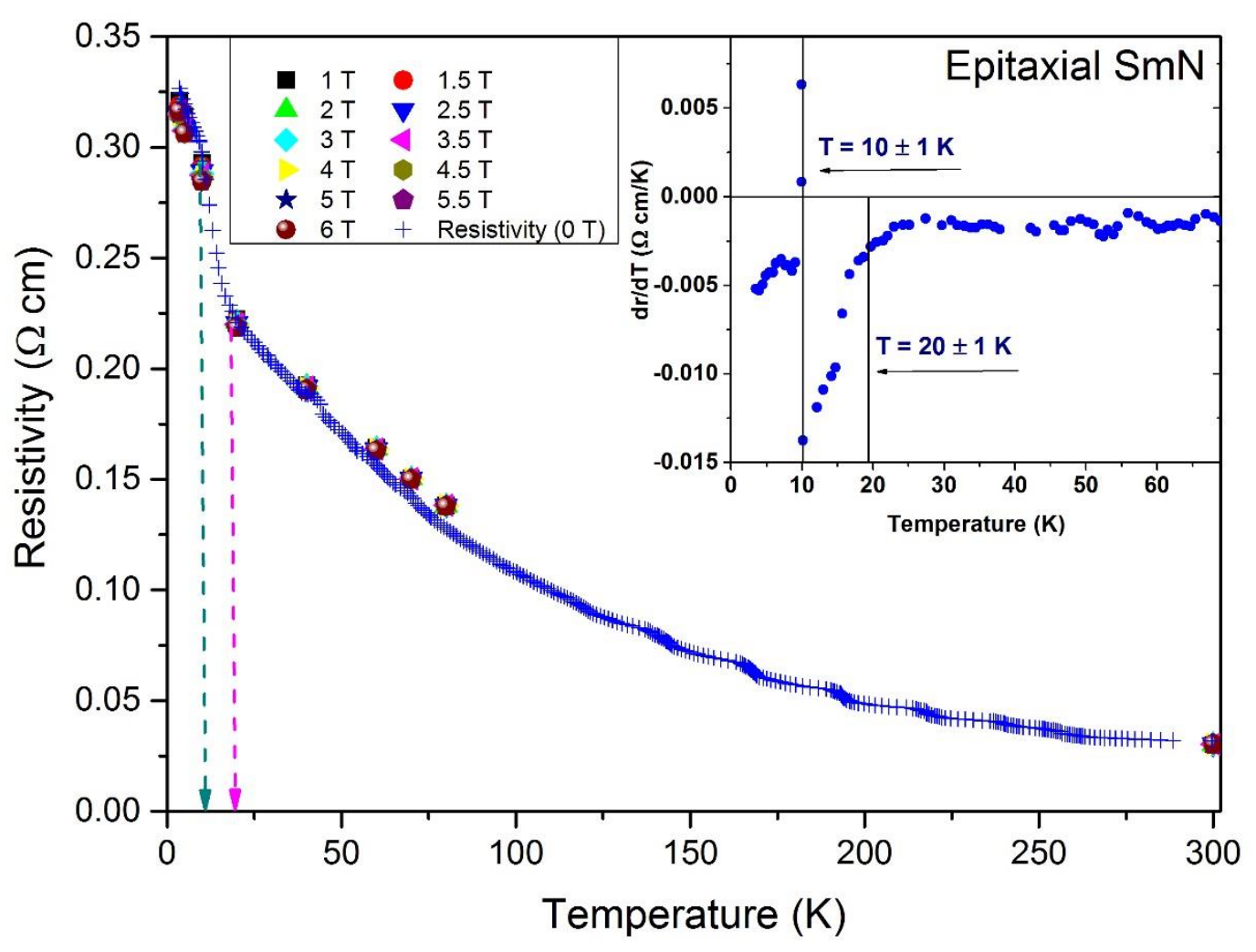

Figure 8.1: Temperature dependent resistivity of the epitaxial $\mathrm{SmN}$ film. The inset shows the temperature derivative of the resistivity.

Figure 8.1 shows the resistivity as a function of temperature and the influence of magnetic field on the resistivity results at various temperatures. The resistivity experiment was performed between $300 \mathrm{~K}$ to $2 \mathrm{~K}$ temperature regions whereas field dependent resistivity was carried out in the presence of $\pm 6 \mathrm{~T}$ magnetic field. The resistivity increases as temperature decreases and shows a steep rise below $20 \mathrm{~K}$. There is a transition at low temperature. This deviates from a nice curve when we reached $20 \mathrm{~K}$ as shown in the inset figure. It is to be noted that there is a discontinuity near this temperature and possible $T_{c}$ is $20 \mathrm{~K}$ in this sample but we do not have the magnetisation data to confirm it. Interestingly, we have seen a steep rise near $10 \mathrm{~K}$ which is again prominent in the temperature derivative of the resistivity. This temperature could be 
related to a double transition temperature of this film. Some $\mathrm{SmN}$ films show the resistivity peak like GdN films but it is clearly absent in the present case [20].

The magnetoresistance is very small in the present case. This is due to a weak Zeeman term $(\vec{m} . \vec{B})$ as $\mathrm{m}$ is very small. So one expects the applied magnetic field to have a much weaker effect on this material and indeed it does as shown in Figure 8.1.

Next to the resistivity, we have carried out the Hall effect experiment as a function of temperature and field in order to obtain the carrier concentration of the film. The experiment was performed between $80 \mathrm{~K}$ to $10 \mathrm{~K}$ temperature region in the presence of $\pm 6 \mathrm{~T}$ magnetic field. There are no data obtained at room temperature due to a contact issue. Unlike the epitaxial GdN films, here we have faced difficulties to perform this experiment and extract the carrier concentration from the inverse of the slope due to the Hall effect slopes were noisy as prominent in Figure 8.2. As stated earlier in Chapter 4 (Section 4.2.4), this type of noisy data can be seen if one of the contacts are not in the perfectly square position in the van der Pauw geometry which thus produce the high offset Hall voltage.

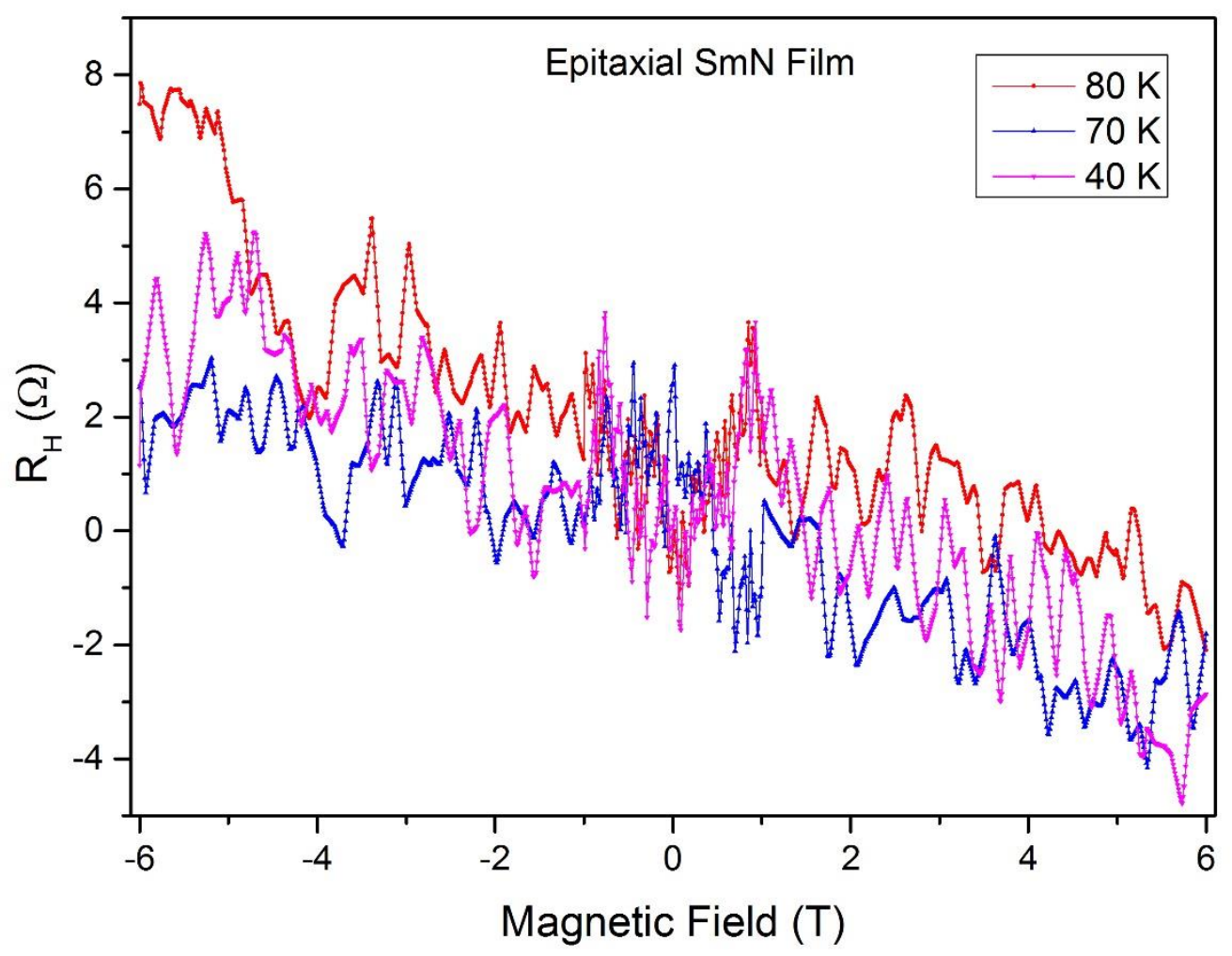

Figure 8.2: Hall resistance as a function of applied magnetic field for the epitaxial $\mathrm{SmN}$ film. 
The negative gradient of the Hall effect suggested that the charge carriers are electron as expected for n-type materials. Figure 8.3 shows the inverse of the slope as a function of temperature. The carrier concentration extracted from the inverse of the slope is found to be nearly constant below $100 \mathrm{~K}$ and it is around $1.2 \pm 0.5 \times 10^{20} / \mathrm{cm}^{3}$. The carrier concentration is smaller than the $10^{21} / \mathrm{cm}^{3}$ which we often get in this material. A weak variation in carrier concentration was seen over the range of $\mathrm{SmN}$ films typically grown in this condition suggests the conductivity is dominated by a simple single-band model.

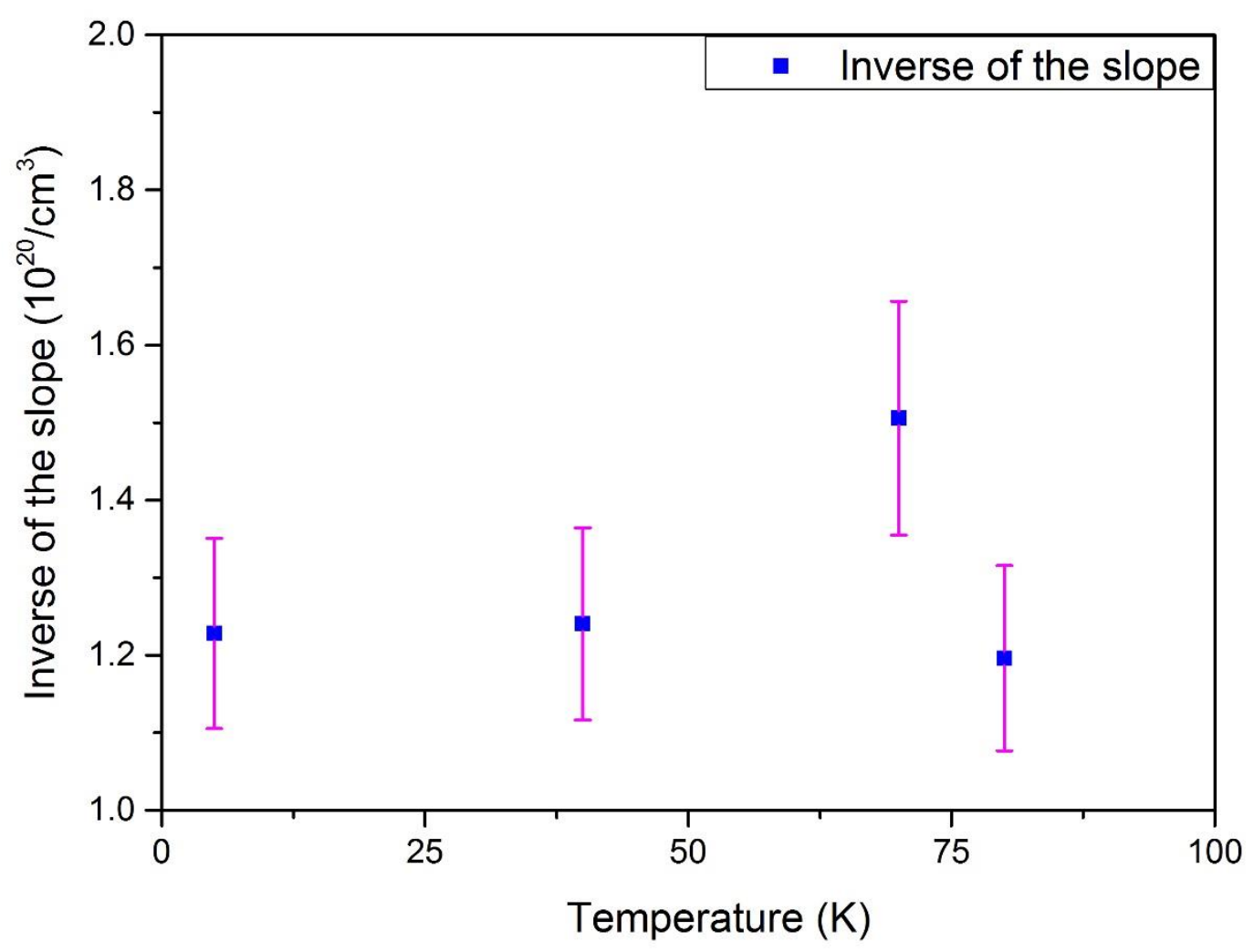

Figure 8.3: Inverse of the slope of the epitaxial $\mathrm{SmN}$ film at different temperatures. 


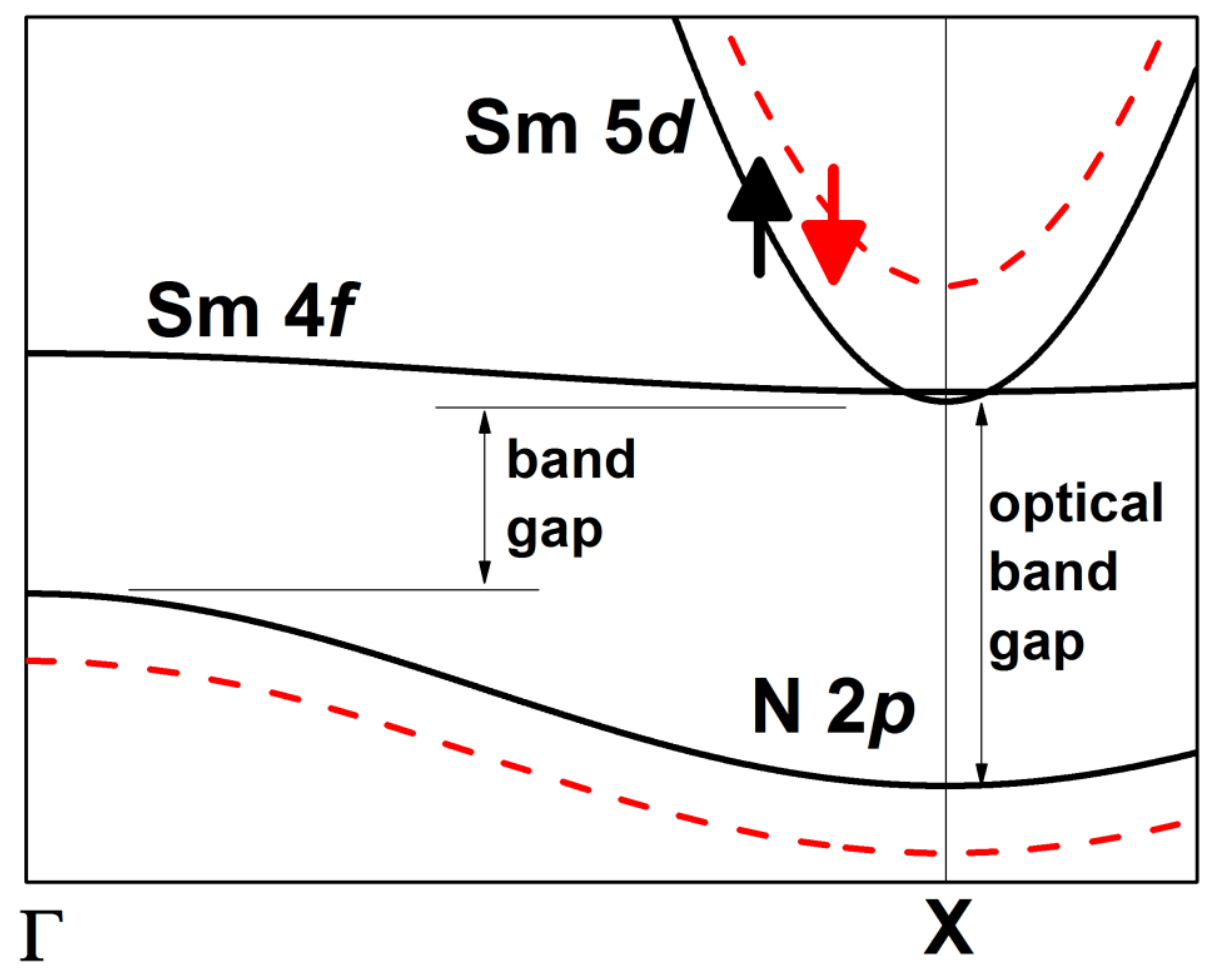

Not to scale

Figure 8.4: The schematic band structure pattern of SmN film drawn from the reference [25].

We have tried to understand our results based on the schematic band structure shown in Figure 8.4. This simplified figure is drawn from the reference [25] which was actually produced from the LSDA $+U$ calculation. Note that there is a big difference with the band structure of GdN and $\mathrm{SmN}$ in terms of the $4 f$ level. Unlike the $\mathrm{GdN}$ films, here $4 f$ bands may play an important role in the conduction process. The conduction band of SmN has two empty majority-spin $4 f$ bands, in addition to the $5 d$ band. It is quite possible that $4 f$ bands hybridized with the $5 d$ band or lie lower than the $5 d$ band and the conduction process occurs via this channel $(4 f)$. This feature is supported by the superconductivity nature of $\mathrm{SmN}$ published by our research group [126]. The minority-spin $4 f$ band lies somewhere $5 \mathrm{eV}$ higher, which again ensures that the conduction process is dominated by the majority-spin electrons in either band. 


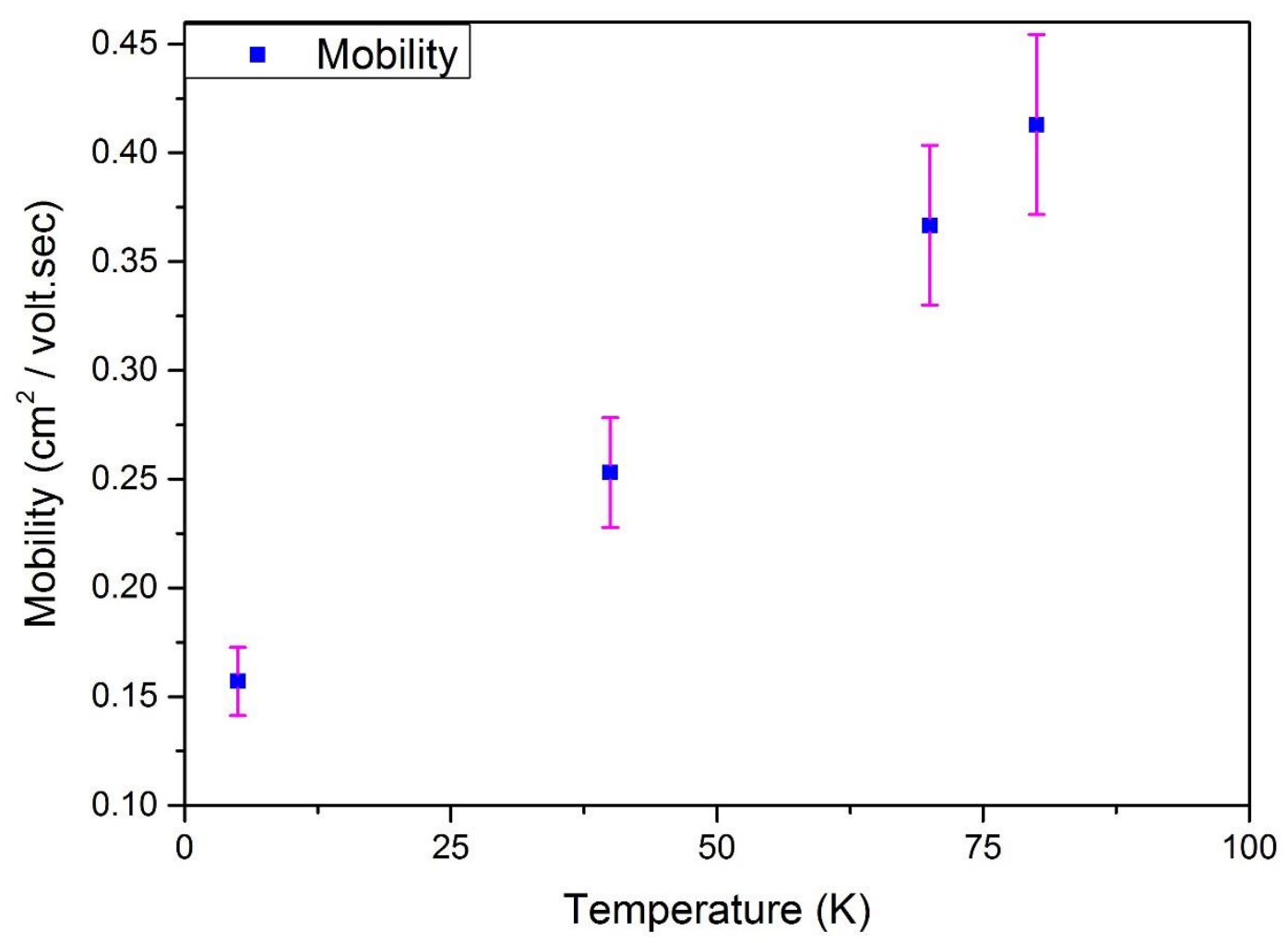

Figure 8.5: Mobility as a function of temperature of the epitaxial $\mathrm{SmN}$ film.

Figure 8.5 shows mobility as a function of temperature. Mobility increases with the increment of temperature from lower temperature up to $80 \mathrm{~K}$. Nonetheless one might expect that if we raise the temperature above $T_{c}$ then we will get the smaller mobility. In the present case, the mobility rises about a factor of 3 from the lower temperature which suggests the sample is already above the Curie temperature and the magnetic fluctuation reduces. This would also suggest the lower Curie temperature of this sample. 


\subsubsection{Magnetoresistance results}

Transport measurements have been further investigated by doing magnetoresistance measurements. Figures 8.6 and 8.7 show magnetoresistance of the epitaxial SmN film in applied fields perpendicular and parallel to the film surface, respectively. The experiment was performed at different temperatures, ranging from $80 \mathrm{~K}-3.5 \mathrm{~K}$.

A magnetic field reduces the magnetic fluctuation as also seen in GdN films. The maximum decrement of resistance is $4 \%$ at $5 \mathrm{~K}$. The magnitude of MR is much smaller than the reported GdN films in this thesis as one would expect.

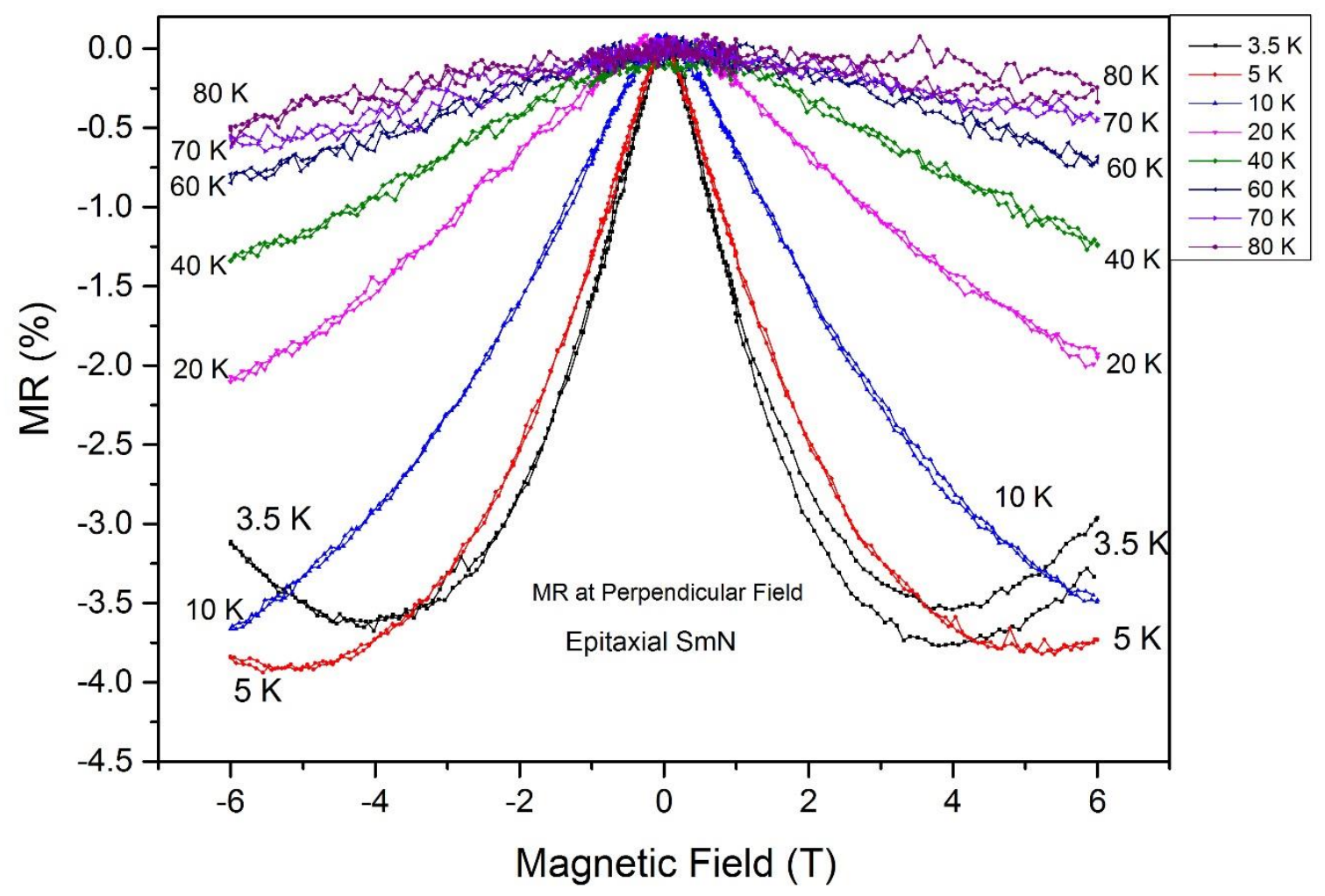

Figure 8.6: Magnetoresistance for the epitaxial $\mathrm{SmN}$ film in applied fields perpendicular to the film surface at different temperatures. 


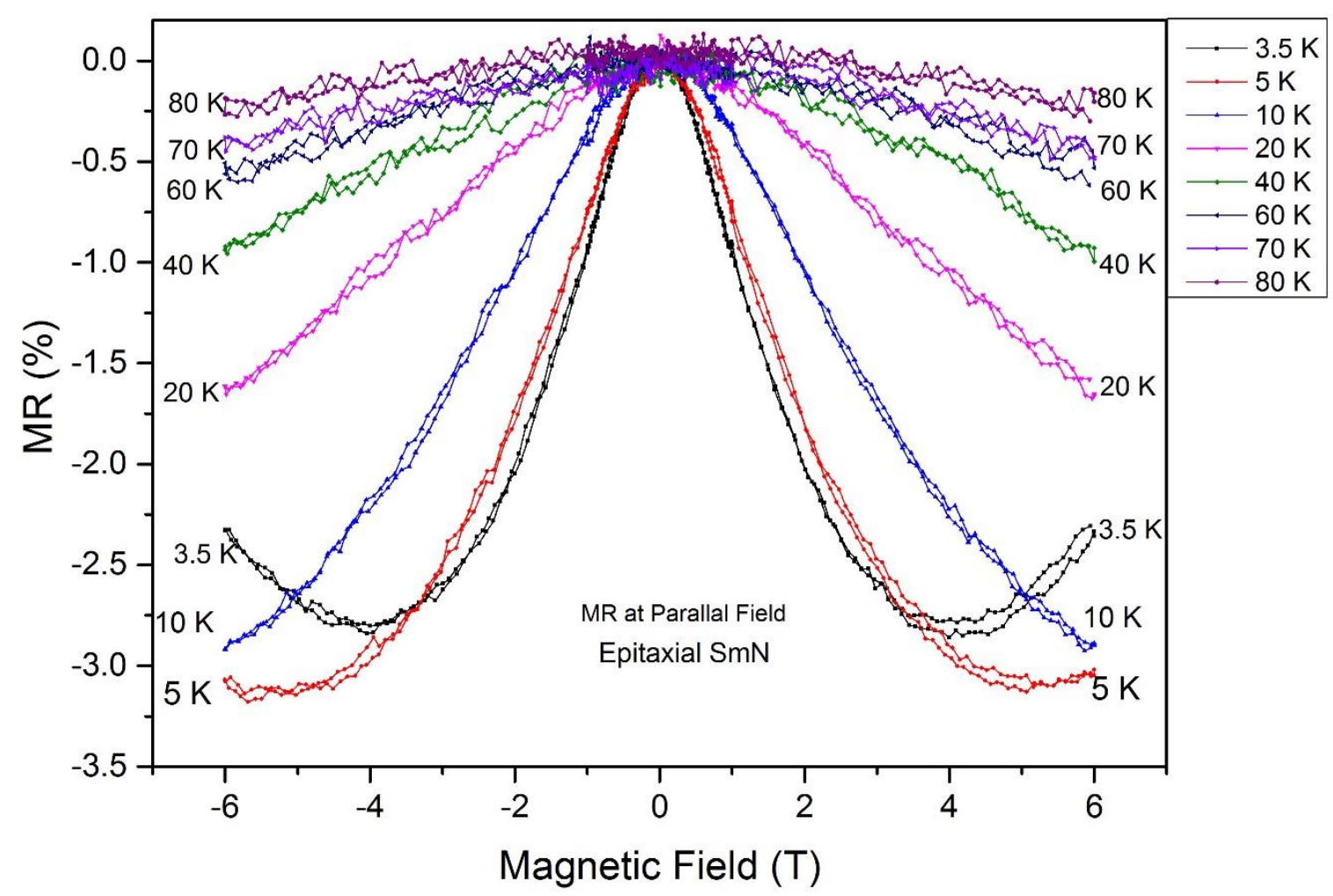

Figure 8.7: Magnetoresistance for the epitaxial $\mathrm{SmN}$ film in applied fields parallel to the film surface at different temperatures. 


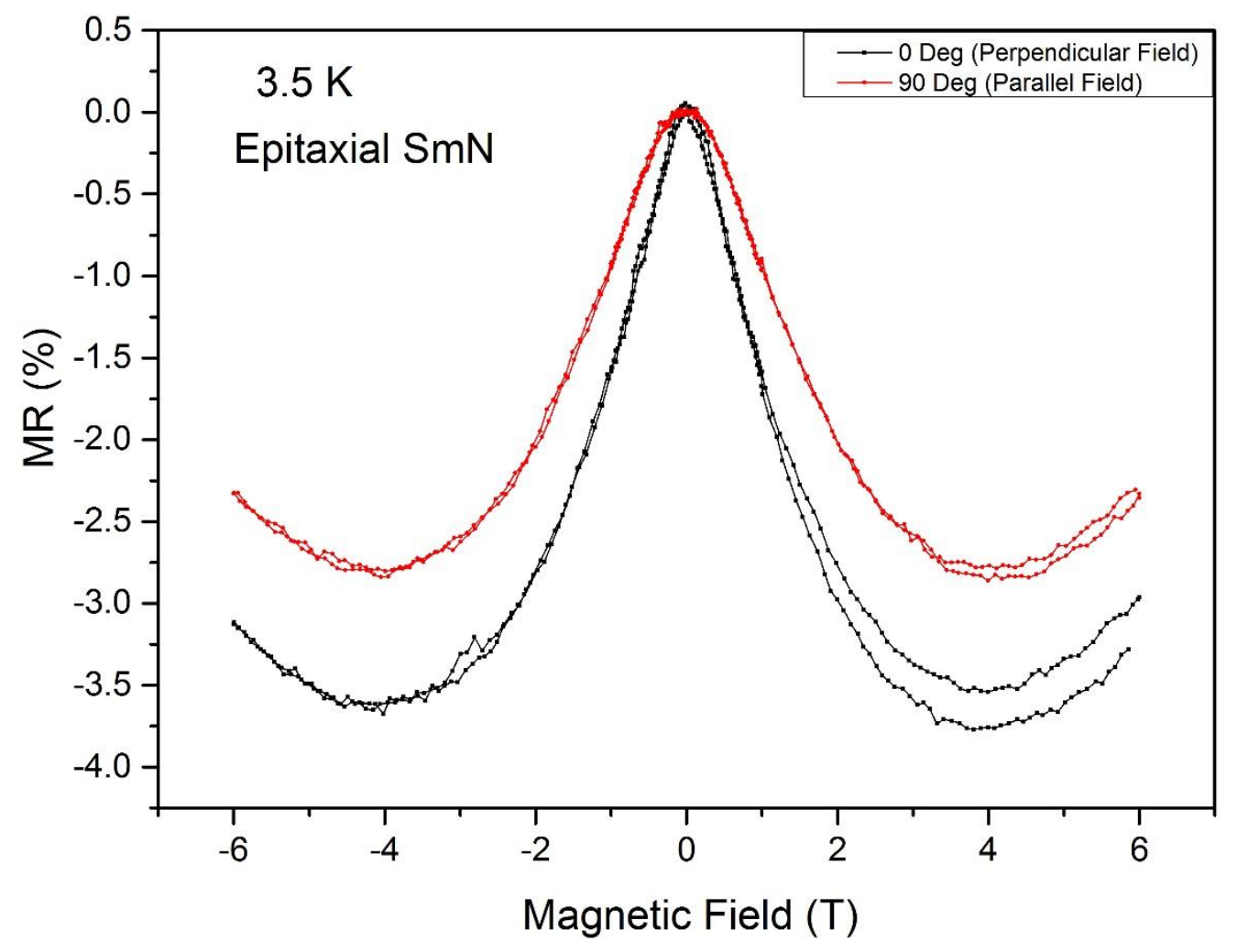

Figure 8.8: Magnetoresistance of the epitaxial $\mathrm{SmN}$ film at $3.5 \mathrm{~K}$ measured with the applied magnetic field perpendicular (black line) and parallel (red line) to the film surface.

MR results are not even saturated in the presence of a highest applied magnetic field of $\pm 6 \mathrm{~T}$. Again this is because the magnetisation is very small for $\mathrm{SmN}$ and needs large fields to demagnetised the film [125]. For $3.5 \mathrm{~K}$ temperature at $\pm 4 \mathrm{~T}$ magnetic fields MR results show upturning nature. We speculate that it has to do with the fact that the conventional positive MR eventually becomes dominated at high fields and low temperatures.

The width of the MR curve is narrower in the field perpendicular case, exactly the opposite what one would expect from shape anisotropy. However, the shape anisotropy is unimportant in $\mathrm{SmN}$ because magnetisation is found to be very small for SmN. The field perpendicular application is along the crystallographic easy axis. This film is predominately [100] which now suggests the easy axis is [100]. 


\subsubsection{Thermopower results of the epitaxial and polycrystalline SmN films}

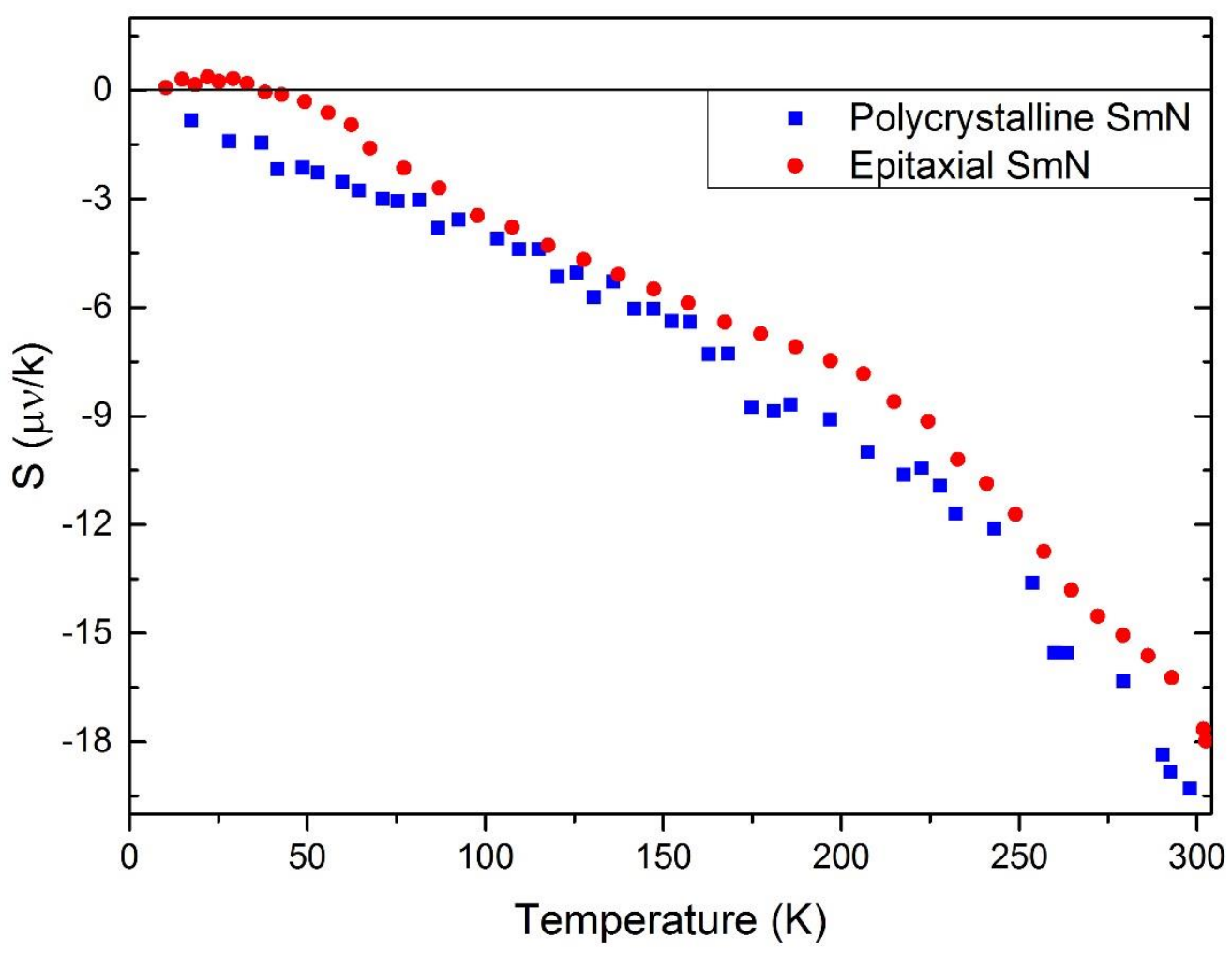

Figure 8.9: Temperature dependent thermopower results of the epitaxial $\mathrm{SmN}$ film.

Figure 8.9 shows the thermopower results collected on $\mathrm{SmN}$ films, i.e. one epitaxial and one polycrystalline SmN film respectively. We have seen the remarkable agreement between these films. In both cases, the thermopower sign agrees with electron doping of the samples similar to the GdN film. The magnitude of thermopower is smaller than the epitaxial GdN_HC film. For the epitaxial $\mathrm{SmN}$ film thermopower changes sign below $30 \mathrm{~K}$ as also seen in $\mathrm{GdN}$ and approaches to zero as temperature reduces to zero as expected.

For the polycrystalline $\mathrm{SmN}$ film, thermopower changes quite linearly with temperature in some regions with marginally larger slope than epitaxial GdN film. The crude estimation of the Fermi energy calculated from the diffusion thermopower slope for the epitaxial and polycrystalline $\mathrm{SmN}$ films are approx. $1 \mathrm{eV}$ and $0.7 \mathrm{eV}$, respectively, quite close to the $\mathrm{GdN}$ 
film. These results suggest a presence of moderate carrier concentration. The high carrier concentration (from the Hall effect measurement) and Fermi energy indicate both films are moderately doped semiconductors. We would like to note that the difficult band structure of SmN, especially the presence of $4 f$ level near the bottom of the conduction band complicates all of these interpretations and as a consequence more work is required.

\subsection{Summary and conclusion}

In this chapter, we have presented preliminary transport results of an epitaxial SmN film and thermopower result of a polycrystalline $\mathrm{SmN}$ film. Our early results point to new measurements that can be made. The resistivity shows a typical semiconducting nature of the epitaxial SmN film. The result shows a bump near the $T_{c}$, lower than the expected $T_{c}$ of $27 \mathrm{~K}$. A further anomaly is seen near $10 \mathrm{~K}$ which one could argue about the double transition temperature like GdN film. Unlike the GdN films, the resistivity of the film is not suppressed much in the presence of magnetic fields due to weak Zeeman alignment. The Hall effect results suggest a nearly constant carrier concentration below $80 \mathrm{~K}$. We calculated the mobility and surprisingly it increases as temperature rises above $30 \mathrm{~K}$ but it is quite likely associated with the fact that as we go to higher temperatures, spin fluctuation scattering gets weaker.

The thermopower results are found to be smaller than in the epitaxial GdN_HC film and remarkable agreement seen between epitaxial and polycrystalline $\mathrm{SmN}$ films. The thermopower sign agrees with electron doping of the samples which is also supported by the Hall effect results (negative slope). The high Fermi energy crudely estimated from diffusion thermopower slopes suggest that Fermi level lies above the bottom of the conduction band in both cases. In conclusion, both $\mathrm{SmN}$ films are moderately doped semiconductors. 


\section{Chapter 9}

\section{Summary and future work}

\subsection{Summary}

This thesis presents a study of the electron and thermal transport properties of rare-earth nitride films to advance the state of knowledge by using a range of complementary techniques. The attention was focused on two interesting RENs, i.e. GdN and SmN. A detailed investigation of transport properties along with the structural and magnetic properties was presented on $\mathrm{GdN}$ films, while preliminary transport measurements were performed on $\mathrm{SmN}$ films. Our results point to a description of films with different carrier concentration which is discussed in four results chapters. The overall experimental results of this thesis are summarised here.

The charge and heat transport measurements have been performed thoroughly on the GdN_HC film. The experimental results then compared with the band structure based on a LSDA $+U$ treatment. In the present case the nitrogen vacancy creates a resonant state in the conduction band and all free carriers reside in the conduction band. The conductivity of this film is dominated by the majority-spin pockets in the conduction band of GdN.

Extended conventional transport properties investigated the thermoelectric power of this film giving an estimation of the Fermi energy which is reasonable and gives the sign which agrees with the Hall effect. We have noticed that at low temperature the thermopower changes in sign and it is interpreted in terms of the strong temperature dependence of the scattering frequency. This finding is quite unique and it is absolutely new. The large number of charge carriers and high Fermi energy of this film suggest that this film is a better representative of a heavily doped film with a metallic characteristic. 
Our research extended further to the low conductivity GdN_LC film. The Hall effect results of this film are quite unique and interpreted using the SQUID results in the ferromagnetic region and the Brillouin function model in the paramagnetic region. We found that Hall effect slopes are strongly influenced by magnetisation of the film which has not been identified or reported by any other research group in this field so far. The carrier concentration of this film is temperature independent which is a typical characteristic of a metallic film. Its metallic nature is further implied by the high carrier concentration of $\sim 10^{20} / \mathrm{cm}^{3}$ and the conductivity is dominated by the majority-spin band.

The polycrystalline film showed the lowest conductivity among all GdN films reported in this thesis. The room temperature Hall effect data shows that the carrier concentration is lower than both of the epitaxial films and insufficient to overfill the majority band. We have seen two new temperature phenomena in the current result; just above and below $15 \mathrm{~K}$. Below $15 \mathrm{~K}$, the result has been interpreted based on thermal activation behaviour. The thermal activation behaviour becomes flat above this temperature once polarons begin to overlap. As a consequence, free carriers are found to be delocalised in the impurity band and the conduction process then occurs through this channel.

This polycrystalline GdN film showed the highest negative MR value so far reported in the literature for REN films. The magnitude of MR for the polycrystalline film is significantly bigger than the epitaxial GdN films and it is nearly $70 \%$ in a field of $8 \mathrm{~T}$ at $60 \mathrm{~K}$. Our results suggest that in the presence of an applied field the magnetic fluctuations reduce significantly which therefore reduce the scattering rate and enhance the conductivity of the film.

This film shows more of metallic nature above $15 \mathrm{~K}$ where carrier concentration are expected to be approximately fixed and found to be of a semiconducting nature below this temperature. In conclusion, this film is a better representation of a weakly doped semiconductor.

The magnetotransport results of $\mathrm{SmN}$ found that the resistivity of the film does not suppress much in the presence of magnetic field because of a weak Zeeman term. The MR results suggest the easy axis of this film is [100], which is one of the more notable discoveries in the present work. 
The extended transport measurements studied the thermopower on this film and a polycrystalline SmN film. The results show the remarkable agreement between epitaxial and polycrystalline SmN films. The thermopower sign agrees with electron doping of the samples as expected. The crude estimation of the Fermi energy calculated from the diffusion thermopower slope is approx. $1 \mathrm{eV}$ which suggests that the Fermi level lies above the bottom of the conduction band. The conduction band of $\mathrm{SmN}$ is comprised of $4 f$ bands and $5 d$ bands. It is quite possible that $4 f$ bands hybridized with the $5 d$ band and the conductivity of this film is dominated by the majority-spin electrons in either band. We conclude that both SmN films are moderately doped semiconductors.

The magnetotransport results, i.e. magnetic, resistivity, Hall effect, magnetoresistance and thermopower have been discussed in terms of a reasonable model for the conduction in $\mathrm{GdN}$ and SmN. Some of our results on GdN film have already been published where other results are on the way to be published. The overall results suggest that the nitrogen vacancy concentration controls the carrier concentration which therefore changes the transport properties of these materials. We have nicely correlated all our experimental results within the existing band structure theory based on a LSDA $+U$ treatment and found that all films are either heavily, moderately or weakly doped semiconductors with a metallic characteristic.

The following are our unique key findings from this research project:

1. For epitaxial GdN films, carrier concentrations are so high that the nitrogen vacancies create a resonant state within the conduction band and the Fermi level lies inside the conduction band.

2. We found that thermal activation behaviour, the most commonly used to interpret resistivity, is not applicable.

3. The polarons can overlap and the conduction process occurs through the impurity band.

4. A resistivity peak appears due to magnetic scattering.

5. The magnetoresistance is high and can be further enhanced by adjusting the growth conditions.

6. The conductivity of all films are mostly dominated by the majority-spin single-band.

7. The anomalous Hall effect can be present even in the paramagnetic region as seen in the epitaxial GdN_LC film. 
8. Magnetisation of films can affect the Hall resistance slope which needs to correct first for calculating the carrier concentration from the slope.

9. The thermopower results indicate electron carriers as supported by the Hall effect measurement.

10. The thermopower results give a crude estimation of the Fermi energy which was not previously obtained experimentally on REN films.

\subsection{Outlook}

Over the last decade research on RENs has significantly improved towards developing novel spintronics devices. The recent high-quality sample preparation techniques, particularly applied to $\mathrm{GdN}$ and $\mathrm{SmN}$ films, provide more reliable transport and magnetic results which are comparable with the theoretical results obtained by advanced density functional theory. Future research progress toward determining the electronic and magnetic structure of all other RENs will depend largely on the availability of high-quality films. Hence, further improvement of film growth techniques is one of the current research requirements in this field. Interestingly, research on these materials continues to grow. In spite of our greater understanding of $\mathrm{GdN}$ and $\mathrm{SmN}$, the overall study of other members of the RENs is incomplete. Especially, the understanding of the origin of the intrinsic anomalous Hall effect is still an open question. Therefore, much more work is required to completely understand the transport, magnetic and optoelectronic properties of all REN candidates.

As this thesis has mainly reported the results of $\mathrm{GdN}$ and $\mathrm{SmN}$ films, we have highlighted some relevant future works on these materials for our better understanding.

First of all, more careful studies should be performed on transport properties as these properties significantly depend on the growth temperature which in turn controls the nitrogen vacancy concentration. Secondly, the polycrystalline film shows a large magnetoresistance which may be useful as a magnetic detector. This calls for further investigation of the magnetoresistance in polycrystalline samples in order to optimise the magnetoresistance sensitivity. 
In this thesis we have made only an initial investigation of thermopower measurements. The result is consistent with other experimental data, nonetheless a detailed understanding is still lacking, in particular how the diffusion thermopower slope depends on carrier concentration. Furthermore the change in sign at low temperatures needs to be investigated more thoroughly. One could also investigate the possibility of the presence of phonon drag at low temperature and that would have to be done by developing a better understanding of the scattering phenomena, i.e. one might distinguish the individual contribution of phonon drag and magnetic fluctuations from the low temperature thermopower result. Thermopower experiments also need to be investigated as a function of magnetic field and it would be worthwhile to correlate the magneto-thermopower and magnetoresistance results.

Therefore systematic investigation and more experimental work are required to untangle the understanding of these materials. There is an enormous opportunity ahead, and a good chance that the RENs explored within this thesis could play a significant role in future technology and upgrade current spintronics devices. Hopefully the new results contained in this thesis will contribute to these advances. 


\section{Appendix A}

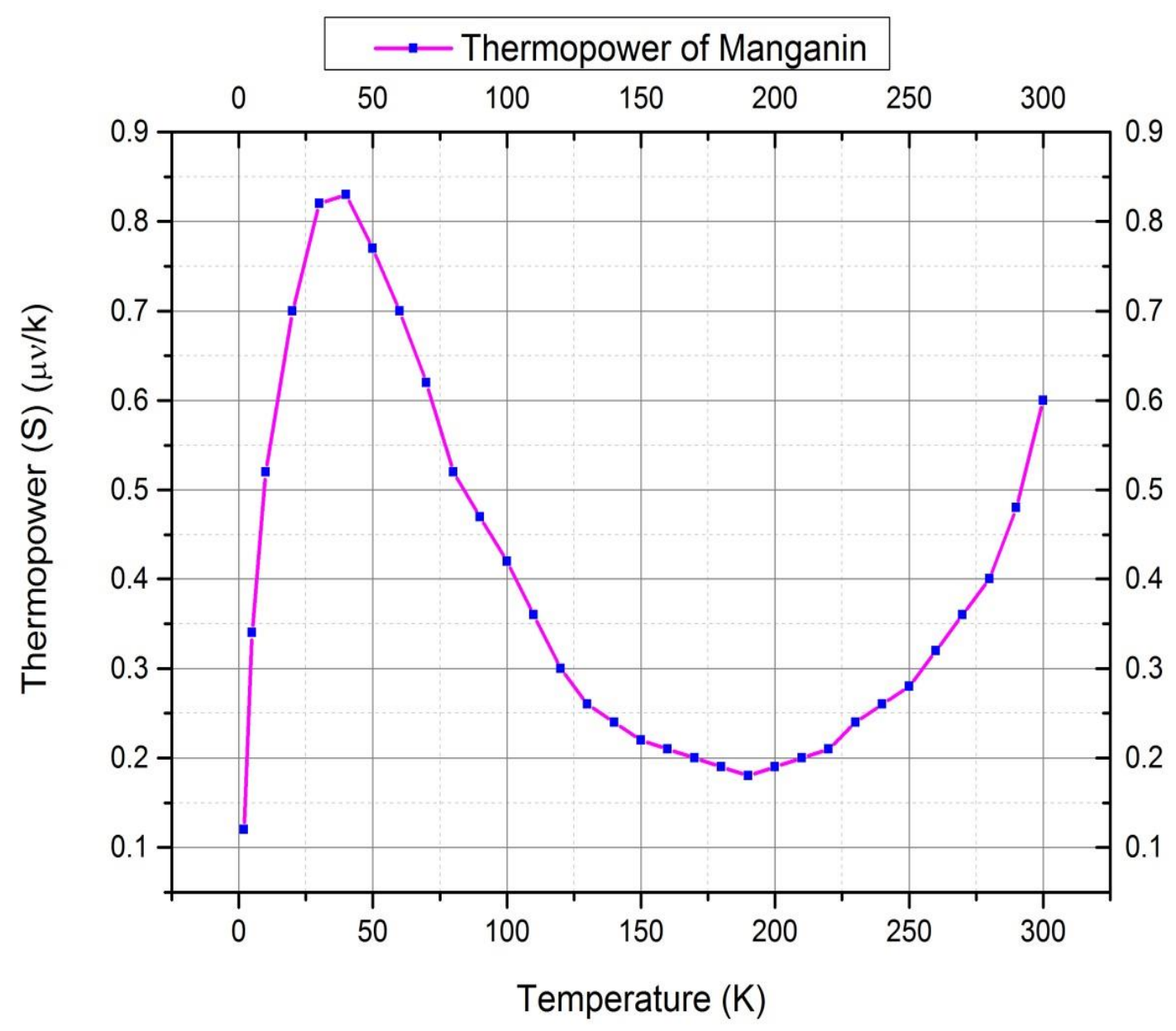

Figure A.1: Thermopower of manganin at various temperatures (Reproduced from [172]). 


\section{Appendix B}

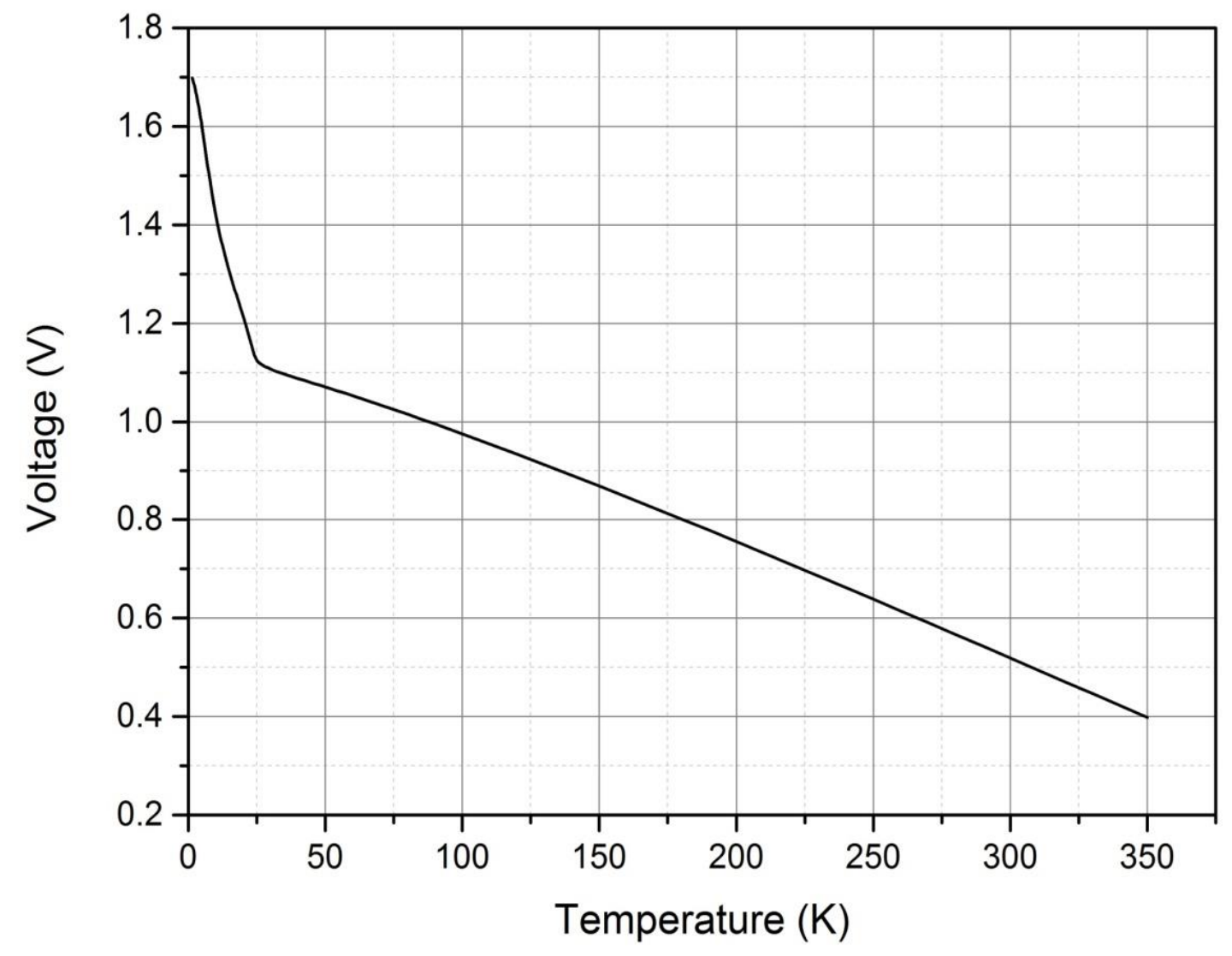

Figure B.1: Voltage and Temperature relationship for DT $470 \mathrm{CU}$ thermometer for the thermopower measurement (Adapted from [170]). 


\section{References}

[1] S. D. Bader, “Colloquium: Opportunities in nanomagnetism”, Rev. Mod. Phys., vol. 78, 1, (2006).

[2] I. Žutić, J. Fabian and S. D. Sarma, "Spintronics: Fundamentals and applications", Rev. Mod. Phys., vol. 76, 323, (2004).

[3] T. Dietl, H. Ohno, F. Matsukura, J. Cibert and D. Ferrand, "Zener Model Description of Ferromagnetism in Zinc-Blende Magnetic Semiconductors”, Science., vol. 287, 1019, (2000).

[4] J. K. Furdyna, "Diluted magnetic semiconductors", J. Appl. Phys., vol. 64, 29, (1988).

[5] H. Munekata, H. Ohno, S. von Molnar, A. Segmüller, L. L. Chang and L. Esaki, “Diluted magnetic III-V semiconductors”, Phys. Rev. Lett., vol. 63, 1849, (1989).

[6] Ohno, H., H. Munekata, T. Penney, S. von Molnár and L. L. Chang, "Magnetotransport Properties of p-Type (In,Mn)As Diluted Magnetic III-V Semiconductors”, Phys. Rev. Lett. vol. 68, 2664, (1992).

[7] H.Ohno, A. Shen, F. Matsukura, A. Oiwa, A.Endo, S. Katsumoto and Y. Iye, "(Ga,Mn)As: A new diluted magnetic semiconductor based on GaAs", Appl. Phys. Lett., vol. 69,363 , (1996).

[8] H. Ohno, "Making Nonmagnetic Semiconductors Ferromagnetic”, Science, vol. 281, 951, (1998).

[9] T. Jungwirth, K. Y. Wang, J. Masek, K. W. Edmonds, J. Konig, J. Sinova, M. Polini, N. A. Goncharuk, A. H. MacDonald, M. Sawicki, A. W. Rushforth, R. P. Campion, L. X. Zhao, C. T. Foxon and B. L. Gallagher, "Prospects for high temperature ferromagnetism in (Ga,Mn)As semiconductors”, Phys. Rev. B., vol. 72, 165204, (2005).

[10] T. Dietl, H. Ohno and F. Matsukura, "Hole-mediated ferromagnetism in tetrahedrally coordinated semiconductors”, Phys. Rev. B., vol. 63, 195205, (2001). 
[11] G. T. Thaler, M. E. Overberg, B. Gila, R. Frazier, C. R. Abernathy, S. J.Paerton, J. S. Lee, S. Y. Lee, Y. D. Park, Z. G. Khim, J. kim and F. Ren, "Magnetic properties of nGaMnN thin films", Appl. Phys. Lett., vol. 80, 3964, (2002).

[12] S. Dhar, O. Brandt, M. Ramsteiner, V. F. Sapega and K. H. Ploog, "Colossal Magnetic Moment of Gd in GaN”, Phys. Rev. Lett., vol. 94, 037205, (2005).

[13] M. Venkatesan, C. B. Fitzgerald, J. G. Lunney and J. M. D. Coey, “Anisotropic Ferromagnetism in Substituted Zinc Oxide”, Phys. Rev. Lett., vol. 93, 177206, (2004).

[14] T. Dietl and H. Ohno, "Dilute ferromagnetic semiconductors: Physics and spintronic Structures”, Rev. Mod. Phys., vol. 86, (2014).

[15] B. J. Ruck, "Spintronics potential of rare earth nitrides and related materials, in Nanomagnetism and Spintronics”, edited by F. Nasipouri and A. Nogaret (World Scientific), 193, (2009).

[16] T. Jungwirth, Jairo Sinova, J. Mašek, J. Kučera and A. H. MacDonald, “Theory offerromagnetic (III,Mn)V semiconductors”, Rev. Mod. Phys., vol.78, 809, (2006).

[17] T. Dietl, "A ten-year perspective on dilute magnetic semiconductors and oxides", Nat. Mater. vol. 9, 965, (2010).

[18] Jacek Kossut and Jan A.Gaj, "Introduction to the Physics of Diluted Magnetic Semiconductors”, Springer Series in materials science, Springer, 144, (2010).

[19] C. M. Aerts, P. Strange, M. Horne, W. M. Temmerman, Z. Szotek and A. Svane, "Halfmetallic to insulating behaviour of rare-earth nitrides”, Phys. Rev. B., vol. 69, 045115, (2004).

[20] A. R. H. Preston, S. Granville, D. H. Housden, B. Ludbrook, B. J. Ruck, H. J.Trodahl, A. Bittar, G. V. M. Williams, J. E. Downes, A. DeMasi, Y. Zhang, K. E.Smith and W. R. L. Lambrecht, "Comparison between experiment and calculated band structures for $D y N$ and $S m N ”$, Phys. Rev. B. vol. 76, 245120, (2007).

[21] F. Hulliger, "Handbook on the Physics and Chemistry of Rare Earths", vol. 4, chapter edited by Karl A. Gschneider Jr. and Le Roy Eyring, 153, North-Holland Physics Publishing, New York, (1979). 
[22] O. Vogt and K. Mattenberger, "Handbook on the Physics and Chemistry of Rare Earths", chapter edited by K. A. Gschneider Jr., L. Eyring, G. H. Lander and G. R. Choppin, Elsevier Science Publishers, New York, vol. 17, 301, (1993).

[23] D. X. Li, Y. Haga, H. Shida, T. Suzuki and Y. S. Kwon, "Electrical transport properties of semimetallic GdX single crystals ( $X=P, A s, S b$ and $B i$ )”, Phys. Rev. B., vol. 54, 10483, (1996).

[24] F. Natali, B.J. Ruck, N.O.V. Plank, H.J. Trodahl, S. Granville, C. Meyer and W.R.L. Lambrecht, "Rare-earth mononitrides", Progress in Materials Science, vol. 58, 1316, (2013).

[25] P. Larson, W. R. L. Lambrecht, A. Chantis and M. van Schilfgaarde, "Electronic structure of rare-earth nitrides using the LSDA+U approach: Importance of allowing $4 f$ orbitals to break the cubic crystal symmetry”, Phys. Rev. B., vol. 75, 045114, (2007).

[26] C. G. Duan, R. F. Sabiryanov, W. N. Mei, P. A. Dowben, S. S. Jaswal and E. Y. Tsymbal, "Electronic, magnetic and transport properties of rare-earth monopnictides", J. Phys. Condens. Matter., vol. 19, 315220, (2007).

[27] F. Hulliger, “Magnetic properties of the rare earth pnictides”, J. Magn. Magn. Mater., vol. 8, 183, (1978).

[28] N. Sclar, "Energy Gaps of the III-V and the (Rare Earth) - V Semiconductors", J. Appl. Phys., vol. 33, 2999, (1962).

[29] N. Sclar, "Properties of Rare-Earth Nitrides”, J. Appl. Phys., vol. 35, 1534, (1964).

[30] H. R. Child, M. K. Wilkinson, J. W. Cable, W. C. Koehler and E. O. Wollan, "Neutron Diffraction Investigation of the Magnetic Properties of Compounds of RareEarth Metals with Group V Anions”, Phys. Rev., vol. 131, 922, (1963).

[31] G. T. Trammell, "Magnetic Ordering Properties of Rare-Earth Ions in Strong Cubic Crystal Fields”, Phys. Rev., vol. 131, 932, (1963).

[32] F. Holtzberg, T. R. McGuire, S. Methfessel and J. C. Suits, "Effect of Electron Concentration on Magnetic Exchange Interactions in Rare Earth Chalcogenides", Phys. Rev. Lett., vol. 13, 18, (1964). 
[33] F. Holtzberg, T. R. McGuire, S. Methfessel and J. C. Suits, "Ferromagnetism in Rare Earth Group $V_{A}$ and $V I_{A}$ Compounds with $\mathrm{Th}_{3} P_{4}$ Structure”, J. Appl. Phys., vol. 35, 1033, (1964).

[34] F. Holtzberg, T. R. McGuire and S. Methfessel, "Effect of Electron Concentration on Magnetic Properties of EuTe-GdTe”, J. Appl. Phys., vol. 37, 976, (1966).

[35] G. Busch, "Magnetic Properties of Rare-Earth Compounds”, J. Appl. Phys., vol. 38, 1386, (1967).

[36] R. F. Brown, A. W. Lawson and Glen E. Everett, "Magnetocrystalline Anisotropy of Europium Selenide”, Phys. Rev., vol. 171, 559, (1968).

[37] T. R. McGuire, R. J. Gambino, S. J. Pickart and H. A. Alperin, "Magnetic Structure and Exchange Interactions in Cubic Gadolinium Compounds”, J. Appl. Phys., vol. 40, 1009, (1969).

[38] D. B. McWhan, "Effect of Pressure on the Curie Temperature and Volume of GdN", J. Chem. Phys., vol. 44, 3528, (1966).

[39] T. R. McGuire, R. J. Gambino, S. J. Pickart and H. A. Alperin, "Magnetic Structure and Exchange Interactions in Cubic Gadolinium Compounds”, J. Appl. Phys., vol. 40, 1009, (1969).

[40] H. A. Eick, N. C. Baenziger and L. Eyring, "The Preparation, Crystal Structure and Some Properties of SmN, EuN and YbN ”, J. Am. Chem. Soc., vol. 78, 5987, (1956).

[41] D. X. Li, Y. Haga, H. Shida and T. Suzuki, "Magnetic properties of ferromagnetic GdN”, Physica B: Condensed Matter, vol. 199 \& 200, 631, (1994).

[42] P. Wachter and E. Kaldis, "Magnetic interaction and carrier concentration in GdN and $G d N_{1-x} O_{x} "$, Solid State Commun., vol. 34, 241, (1980).

[43] H. Yoshitomi, S. Kitayama, T. Kita, O. Wada, M. Fujisawa, H. Ohta and T. Sakurai, “Optical and ferromagnetic properties of GdN thin films", P. Stat. Solidi (c)., vol. 8, 488, (2011).

[44] S. Granville, B. J. Ruck, F. Budde, A. Koo, D. J. Pringle, F. Kuchler, A. R. H. Preston, D. H. Housden, N. Lund, A. Bittar, G. V. M. Williams and H. J. Trodahl, “Semiconducting ground state of GdN thin films”, Phys. Rev. B., vol. 73, 235335, (2006). 
[45] Do Le Binh, B. J. Ruck, F. Natali, H. Warring, H. J. Trodahl, E.-M. Anton, C. Meyer, L. Ranno, F. Wilhelm and A. Rogalev, "Europium Nitride: A Novel Diluted Magnetic Semiconductor”, Phys. Rev. Lett., vol. 111, 167206, (2013).

[46] C. Meyer, B.J. Ruck, A.R.H. Preston, S. Granville, G.V.M. Williams and H.J. Trodahl, “Magnetic properties of ErN film”, J. Magn. Magn. Mater, vol. 322, 1973, (2010).

[47] D. L. Cortie, J. D. Brown, S. Brück, T. Saerbeck, J. P. Evans, H. Fritzsche, X. L. Wang, J. E. Downes and F. Klose, "Intrinsic reduction of the ordered 4 magnetic moments in semiconducting rare-earth nitride thin films: DyN, ErN and HoN”, Phys. Rev. B., vol. 89, 064424, (2014).

[48] M. Azeem, B. J. Ruck, Binh Do Le, H. Warring, H. J. Trodahl, N. M. Strickland, A. Koo, V. Goian and S. Kamba, “Optical response of DyN”, J. Appl. Phys., vol. 113, 203509, (2013).

[49] P. Wachter, "Physical properties of stoichiometric GdN single crystals", Elsevier, Amsterdam, vol. 2, 90, (2012).

[50] P. Wachter, N.D.Zhigadlo, "Physical properties of stoichiometric CeN single crystals", Elsevier, Amsterdam, vol. 3, 235, (2013).

[51] P. Wachter, “ Physical Properties of Some Stoichiometric Rare Earth Nitride Single Crystals", Advances in Materials Physics and Chemistry, vol. 5, 96, (2015).

[52] C. Duan, R. F. Sabiryanov, J. Liu, W. N. Mei, P. A. Dowben and J. R. Hardy, "Strain Induced Half-Metal to Semiconductor Transition in GdN”, Phys. Rev. Lett., vol. 94, 237201, (2005).

[53] T. Maity, H. J. Trodahl, F. Natali, B. J. Ruck and S. Vézian, “ Electron transport in heavily doped GdN”, Phys. Rev. Materials., vol. 2, 014405, (2018).

[54] R. Marchand, editors: K. A. Gschneidner and L. Eyring, "Handbook on the physics and chemistry of rare earths", Amsterdam: Elsevier, vol. 25, 51, (1998).

[55] P. Larson and W. R. L. Lambrecht, "Electronic structure of Gd pnictides calculated within the LSDA+U approach”, Phys. Rev. B., vol. 74, 085108, (2006).

[56] R. O. Jones and O. Gunnarsson, "The density functional formalism, its applications and prospects”, Rev. Mod. Phys., vol. 61, 689, (1989). 
[57] V. I. Anisimov, J. Zaanen and O. K. Andersen, "Band theory and Mott insulators: Hubbard U instead of Stoner I", Phys. Rev. B., vol. 44, 943, (1991).

[58] E. Burzo, N. Bucur, H. Allmaier and L. Chioncel, "Half-metallic magnetism in lightrare earth nitrides”, J. Optoelectron. Adv. M., vol. 10, 389, (2008).

[59] S. Abdelouahed and M. Alouani, "Calculated electronic properties and structural phase transitions of GdN pnictide under hydrostatic pressure”, Phys. Rev. B., vol. 76, 214409, (2007).

[60] D. B. Ghosh, M. De and S. K. De, "Electronic, magnetic and optical properties of Gd monopnictides: An LDA+U study”, Phys. Rev. B., vol. 72, 045140, (2005).

[61] A. N. Chantis, M. van Schilfgaarde and T. Kotani, "Quasiparticle self-consistent GW method applied to localized 4 felectron systems", Phys. Rev. B., vol. 76, 165126, (2007).

[62] V. I. Anisimov, F. Aryasetiawan and A. I. Lichtenstein, "First-principles calculations of the electronic structure and spectra of strongly correlated systems: the LDA+U method”, J. Phys.: Condens. Matter., vol. 9, 767, (1997).

[63] A. I. Lichtenstein, V. I. Anisimov and J. Zaanen, "Density-functional theory and strong interactions: Orbital ordering in Mott-Hubbard insulators”, Phys. Rev. B., vol. 52, R5467, (1995).

[64] G. Kotliar, S. Y. Savrasov, K. Haule, V. S. Oudovenko, O. Parcollet and C. A. Marianetti, "Electronic structure calculations with dynamical mean-field theory”, Rev. Mod. Phys., vol. 78, 865, (2006).

[65] M. van Schilfgaarde, T. Kotani and S. Faleev, “Quasiparticle Self-Consistent GW Theory”, Phys. Rev. Lett., vol. 96, 226402, (2006).

[66] J. P. Perdew and A. Zunger, "Self-interaction correction to density-functional approximations for many-electron systems”, Phys. Rev. B., vol. 23, 5048, (1981).

[67] W. M. Temmerman, A. Svane, Z. Szotec and H. Winter, "Electronic Density Functional Theory: Recent Progress and New Directions", Plenum, New York, 327, (1997).

[68] S. Bhattacharjee and S. M. Jaya, "Influence of correlation and temperature on the electronic structure of bulk and thin film GdN”, Eur. Phys. J. B., vol. 49, 305, (2006). 
[69] A. Preston, “Electronic Structure of Rare Earth Nitrides”, Ph.D. thesis, Victoria University of Wellington, (2009).

[70] R. P. Martin, “Electronic Structure: Basic Theory and Practical Methods”, Cambridge University Press, (2004).

[71] A. Hasegawa and A. Yanase, "Energy Band Structures of Gd-Pnictides”, J. Phys. Soc. Jpn., vol. 42, 492, (1977).

[72] A. G. Petukhov, W. R. L. Lambrecht and B. Segall, “Electronic structure of rare-earth pnictides”, Phys. Rev. B., vol. 53, 8, (1996).

[73] P. Larson and W. R. L. Lambrecht, "Electronic structure and magnetism of europium chalcogenides in comparison with gadolinium nitride”, J. Phys.: Cond. Matt., vol. 18, 11333, (2006).

[74] G. Busch, P. Junod, O. Vogt and F. Hulliger, "Ferro-and metamagnetism of rare earth compounds”, Phys. Lett., vol. 6, 79, (1963).

[75] D. X. Li, Y. Haga, H. Shida, T. Suzuki, Y.S. Kwon and G. Kido, "Magnetic properties of stoichiometric Gd monopnictides”, J. Phys.: Cond. Matt., vol. 9, 10777. (1997).

[76] J. Rhyne and T. R. McGuire, "Magnetism of rare-earth elements, alloys and compounds”, IEEE Transactions on Magnetics, vol. 8, 105, (1972).

[77] P. Wachter, E. Kaldis and R. Hauger, "Magnetic Exchange Interaction in the System GdP-GdS”, Phys. Rev. Lett., vol. 40, 1404, (1978).

[78] M. K. Wilkinson, H. R. Child, J. W. Cable, E. O. Wollan and W. C. Koehler, “ Neutron Diffraction Investigations of the Magnetic Ordering in Rare Earth Nitrides”, J. Appl. Phys., vol. 31, S358 (1960).

[79] David P. Schumacher and W. E. Wallace, "Magnetic Characteristics of Gadolinium, Praseodymium and Thulium Nitrides”, J. Appl. Phys., vol. 36, 984, (1965).

[80] T. Kasuya and A. Yanase, “Anomalous Transport Phenomena in Eu-Chalcogenide Alloys", Rev. Mod. Phys., vol.40, 684, (1968).

[81] R. A. Cutler and A. W. Lawson, "Synthesis and magnetic behavior of GdN", J. Appl. Phys., vol. 46, 2739, (1975). 
[82] G. Travaglini, F. Marabelli, R. Monnier, E. Kaldis and P. Wachter, "Electronic structure of ScN”, Phys. Rev. B., vol. 34, 3876, (1986).

[83] L. F. Schneemeyer, R. B. van Dover and E. M. Gyorgy, "Magnetic behavior of diluted lanthanoid-pnictide systems”, J. Appl. Phys., vol. 61, 3543, (1987).

[84] J. Q. Xiao and C. L. Chien, "Proximity Effects in Superconductor/InsulatingFerromagnet NbN/GdN Multilayers”, Phys. Rev. Lett., vol. 76, 1727, (1996).

[85] T. Kasuya and D.X. Li, "Mechanism of strong ferromagnetism in GdN”, J. Magn. Magn. Mater, vol. 167, L1, (1997).

[86] F. Leuenberger, A. Parge, W. Felsch, K. Fauth and M. Hessler, "GdN thin films: Bulk and local electronic and magnetic properties”, Phys. Rev. B., vol. 72, 014427, (2005).

[87] K. Khazen, H. J. von Bardeleben, J. L. Cantin, A. Bittar, S. Granville, H. J. Trodahl and B. J. Ruck, "Ferromagnetic resonance study of GdN thin films with bulk and extended lattice constants”, Phys. Rev. B., vol. 74, 245330, (2006).

[88] M. Scarpulla, C. Gallinat, S. Mack, J. Speck and A. Gossard, “GdN (111) heteroepitaxy on $\mathrm{GaN}(\mathrm{O} O \mathrm{O} 1)$ by $\mathrm{N}_{2}$ plasma and $\mathrm{NH}_{3}$ molecular beam epitaxy”, Journal of Crystal Growth, vol. 311, 1239, (2009).

[89] R. J. Gambino, T. R. McGuire, H. A. Alperin and S. J. Pickart, "Magnetic Properties and Structure of $G d N$ and $G d N_{1-x} O_{x}$ ”, J. Appl. Phys., vol. 41, 933, (1970).

[90] M. Kuznietz, "Effect of Substitutional Anions on the Magnetic Properties of the Mononitrides of Uranium and Gadolinium”, J. Appl. Phys., vol. 42, 1470, (1971).

[91] H. Yoshitomi, S. Kitayama, T. Kita, O. Wada, M. Fujisawa, H. Ohta and T. Sakurai, “Optical and ferromagnetic properties of GdN thin films", P. Stat. Solidi (c)., vol. 8, 488, (2011).

[92] M. Azeem, “Optical Properties of Rare Earth Nitrides”, Ph.D. thesis, Victoria University of Wellington, (2013).

[93] N. O. V. Plank, F. Natali, J. Galipaud, J. H. Richter, M. Simpson, H. J. Trodahl and B. J. Ruck, "Enhanced Curie temperature in N-deficient GdN", Appl. Phys. Lett., vol. 98, 112503, (2011). 
[94] C. Mitra and W. R. L. Lambrecht, "Magnetic exchange interactions in the gadolinium pnictides from first principles”, Phys. Rev. B., vol. 78, 134421, (2008).

[95] K. Doll, "Electronic structure of GdN and the influence of exact exchange”, J. Phys.: Cond. Matt., vol. 20, 075214, (2008).

[96] C. Duan, R. F. Sabiryanov, W. N. Mei, P. A. Dowben, S. S. Jaswal and E. Y. Tsymbal, "Magnetic ordering in Gd monopnictides: Indirect exchange versus superexchange interaction”, Appl. Phys. Lett., vol. 88, 182505, (2006).

[97] F. Natali, B. J. Ruck, H. J. Trodahl, Do Le Binh, S. Vezian, B. Damilano, Y. Cordier, F. Semond and C. Meyer, "Role of magnetic polarons in ferromagnetic GdN”, Phys. Rev. B., vol. 87, 035202, (2013).

[98] B. T. Thole, P. Carra, F. Sette and G. van der Laan, "X-ray circular dichroism as a probe of orbital magnetization”, Phys. Rev. Lett., vol. 68, 1943, (1992).

[99] Y. Teramura, A.Tanaka, B. T. Thole and T. Jo, "Effect of Coulomb Interaction on the X-Ray Magnetic Circular Dichroism Spin Sum Rule in Rare Earths”, J. Phys. Soc. Jpn., vol. 65, 3056, (1996).

[100] F. Leuenberger, A. Parge, W. Felsch, F. Baudelet, C. Giorgetti, E. Dartyge and F. Wilhelm, "X-ray magnetic circular dichroism at the $G d L_{2,3}$ absorption edges in $G d N$ layers: The influence of lattice expansion”, Phys. Rev. B., vol. 73, 214430, (2006).

[101] V. N. Antonov, B. N. Harmon, A. N. Yaresko and A. P. Shpak, "X-ray magnetic circular dichroism in GdN: First-principles calculations”, Phys. Rev. B., vol. 75, 184422, (2007).

[102] J. Mcnulty, “Twisted Magnetization Phases in Orbital-Dominant Rare-Earth Nitrides”, Ph.D. thesis, Victoria University of Wellington, (2015).

[103] K. Senapati, T. Fix, M E. Vickers, M. G. Blamire and Z. H. Barber, "Magnetic exchange hardening in polycrystalline GdN thin films”, J. Phys.: Cond. Matt., vol. 22, 302003, (2010). 
[104] H. Ohta, M. Fujisawa, F. Elmasry, S. Okubo, Y. Fukuoka, H. Yoshitomi, S. Kitayama, T. Kita and O. Wada, "Ferromagnetic State of GdN Thin Film Studied by Ferromagnetic Resonance”, AIP Conf Proc, vol. 1399, 679, (2011).

[105] B. M. Ludbrook, "The Magnetic Properties of Selected Rare Earth Nitrides Grown by Pulsed Laser Deposition”, Master thesis, Victoria University of Wellington, (2009).

[106] P. Wachter, "Handbook on the Physics and Chemistry of Rare Earths", edited by K. A. Gschneidner and L. Eyring, Elsevier, Amsterdam, vol. 2, 507, (1979).

[107] F. Natali, B. Ludbrook, J. Galipaud, N. Plank, S. Granville, A. Preston, B. L. Do, J. Richter, I. Farrell, R. Reeves, S. Durbin, J. Trodahl and B. Ruck, "Epitaxial growth and properties of GdN, EuN and SmN thin films”, Phys. Status Solidi C., vol. 9, 605, (2012).

[108] F. Natali, S. Vézian, S. Granville, B. Damilano, H. J. Trodahl, E.- M. Anton, H. Warring, F. Semond, Y. Cordier, S. V. Chong and B. J. Ruck, "Molecular beam epitaxy of ferromagnetic epitaxial GdN thin film”, Journal of Crystal Growth, vol. 404, 146, (2014).

[109] M. Ludbrook, J. L. Farrell, M. Kuebel, B. J. Ruck, A. R. H. Preston, H. J. Trodahl, L. Ranno, R. J. Reeves and S. M. Durbin, "Growth and properties of epitaxial GdN", J. Appl. Phys., vol. 106, 063910, (2010).

[110] F. Natali, N. O. V. Plank, J. Galipaud, B. J. Ruck, H. J. Trodahl, F. Semond, S. Sorieul and L. Hirsch, "Epitaxial growth of GdN on silicon substrate using an AlN buffer layer”, Journal of Crystal Growth, vol. 312, 3583, (2010).

[111] H. Yoshitomi, S. Kitayama, T. Kita, O. Wada, M. Fujisawa, H. Ohta and T. Sakurai, “Optical and magnetic properties in epitaxial GdN thin films”, Phys. Rev. B., vol. 83, 155202, (2011).

[112] H. Yoshitomi, R. Vidyasagar, S. Kitayama, T. Kita, H. Ohta, S. Okubo, Y. Fukuoka and T. Sakurai, "Ferromagnetic properties of GdN thin films studied by temperature dependent circular polarized spectroscopy”, Appl. Phys. Lett., vol. 101, 072403, (2012). 
[113] R. Vidyasagar, S. Kitayama, H. Yoshitomi, T. Kita, T. Sakurai and H. Ohta, "Study on spin-splitting phenomena in the band structure of GdN”, Appl. Phys. Lett., vol. 100, 232410, (2012).

[114] K. Senapati, T. Fix, M E. Vickers, M. G. Blamire and Z. H. Barber, "Structural evolution and competingmagnetic orders in polycrystalline GdN film”, Phys. Rev. B., vol. 83, 014403, (2011).

[115] W. R. L. Lambrecht, "Electronic structure and optical spectra of the semimetal ScAs and of the indirect-band-gap semiconductors ScN and GdN”, Phys. Rev. B., vol. 62, 13538, (2000).

[116] H. J. Trodahl, A. R. H. Preston, J. Zhong, B. J. Ruck, N. M. Strickland, C. Mitra and W. R. L. Lambrecht, "Ferromagnetic redshift of the optical gap in GdN", Phys. Rev. B., vol. 76, 085211, (2007).

[117] C. Mitra and W. R. L. Lambrecht, "Calculated interband optical transition spectra of GdN”, Phys. Rev. B., vol. 78, 195203, (2008).

[118] D. P. Schumacher and W. E. Wallace, "Magnetic Characteristics of Some Lanthanide Nitrides”, Inorg. Chem., vol. 5, 1563, (1966).

[119] G. Busch, P. Junod, F. Levy, A. Menth and O. Vogt, "Influence of crystal fields on the magnetic properties of the rare-earth nitrides”, Phys. Lett., vol. 14, 264, (1965).

[120] C. Morari, F. Beiuşeanu, I. Di. Marco, L. Peters, E. Burzo, S. Mican and L. Chionce, “Magnetism and electronic structure calculation of SmN", J. Phys. Condens. Matter., vol. 27, 115503, (2015).

[121] R. M. Moon and W. C. Koehler, “Magnetic properties of SmN”, J. Magn. Magn. Mater, vol. 14, 265, (1979).

[122] E. M. Anton, B. J. Ruck, C. Meyer, F. Natali, H. Warring, Fabrice Wilhelm, A. Rogalev, V. N. Antonov and H. J. Trodahl, "Spin/orbitmoment imbalance in the nearzeromoment ferromagnetic semiconductor SmN”, Phys. Rev. B., vol. 87, 134414, (2013). 
[123] J. F. McNulty, B. J. Ruck and H. J. Trodahl, “On the ferromagnetic ground state of SmN”, Phys. Rev. B., vol. 93, 054413, (2016).

[124] R. Didchenko and F.P. Gortsema, "Some electric and magnetic properties of rare earth monosulfides and nitrides”, J. Phys. Chm. Solids., vol. 24, 863, (1963).

[125] C. Meyer, B. J. Ruck, J. Zhong, S. Granville, A. R. H. Preston, G. V. M. Williams and H. J. Trodahl, “Near-zero-moment ferromagnetism in the semiconductor SmN”, Phys. Rev. B., vol. 78, 174406, (2008).

[126] E.-M. Anton, S. Granville, A. Engel, S. V. Chong, M. Governale, U. Zülicke, A. G. Moghaddam, H. J. Trodahl, F. Natali, S. Vézian and B. J. Ruck, “ Superconductivity in the ferromagnetic semiconductor samarium nitride”, Phys. Rev. B., vol. 94, 024106, (2016).

[127] N. A. Spaldin, "Magnetic Materials - Fundamentals and Applications”, Cambridge University Press, Second Edition, (2011).

[128] S. O. Pillai, "Solid State Physics”, Sixth Edition, New Age Science Ltd. UK, (2010).

[129] F. Hund, “Linienspektren und Periodische System der Elemente”, Berlin, (1927).

[130] S. Blundell, "Magnetism in Condensed Matter”, Oxford University Press, (2001).

[131] B. D. Cullity and C. D. Graham, "Introduction to Magnetic Materials", A John Wiley \& Sons, Second Edition, (2009).

[132] N. W. Ashcroft and N. D. Mermin, "Solid State Physics", Holt, Rinehart and Winston, (1976).

[133] C. Kittel, "Introduction to solid state physics”, Eight Edition, Wiley, 2005.

[134] S. L. Gupta and V. Kumar, "Solid State Physics”, Ninth Edition, K. Nath \& Co., Meerut, (2005).

[135] J. P. Srivastava, "Elements of Solid State Physics”, Second Edition, Phi Learning Pvt. Ltd., (2009).

[136] N. Nagaosa, J. Sinova, S. Onoda, A. H. MacDonald and N. P. Ong, “Anomalous Hall effect”, Rev. Mod. Phys., vol. 82, 1539, (2010). 
[137] T. Jungwirth, J. Wunderlich, V. Novák, K. Olejník, B. L. Gallagher, R. P. Campion, K. W. Edmonds, A. W. Rushforth, A. J. Ferguson and P. Němec, "Spin-dependent phenomena and device concepts explored in (Ga,Mn)As”, Rev. Mod. Phys., vol. 86, 855 , (2014).

[138] H. J. Trodahl, F. Natali and B. J. Ruck, “ Carrier-controlled anomalous Hall effect in an intrinsic ferromagnetic semiconductor”, Phys. Rev. B., vol. 96, 115309, (2017).

[139] J. Inaba and T. Katsufuji, "Large magnetoresistance in spin-and carrier-doped $\mathrm{S}_{r} \mathrm{~T}_{i} \mathrm{O}_{3}$ ”, Phys. Rev. B., vol. 72, 052408, (2005).

[140] P. Majumdar and P. B. Littlewood, "Dependence of magnetoresistivity on chargecarrier density in metallic ferromagnets and doped magnetic semiconductors", Nature, vol. 395, 479, (1998).

[141] R. Hasegawa and C. C. Tsuei, "Kondo Effect in Amorphous Fe-Pd-Si and Co-Pd-Si Alloys”, Phys. Rev. B., vol. 3, 214, (1971).

[142] A. R. Harvey, S. Legvold and D. T. Peterson, "Resistivity and Magnetoresistance of Dilute Solutions of Mn in Cu-Ni Alloys”, Phys. Rev. B., vol. 4, 4003, (1971).

[143] A. Brinkman, M. Huijben, M. Van Zalk, J. Huiben, U. Zeitler, J. C. Maan, W. G. Van Der Weil, G. Rijnders, D. H. A. Blank and H. Hilgenkamp, "Magnetic effects at the interface between non-magnetic oxides", Nat. Mater., vol. 6, 493, (2007).

[144] R. P. Khosla and J. R. Fischer, "Magnetoresistance in degenerate CdS: Localized Magnetic Moments”, Phys. Rev. B., vol. 2, 4084, (1970).

[145] A. C. Hewson, "The Kondo problem to heavy fermions", Cambridge University Press, Cambridge, United Kingdom, (1993).

[146] M. T. Béal Monod and R. A. Weiner, "Negative magnetoresistivity in dilute alloys", Phys. Rev. B., vol. 170, 552, (1968).

[147] A. Schmehl, V. Vaithyanathan, A. Herrnberger, S. Thiel, C. Richter, M. Liberati, T. Heeg, M. Rockerath, L. F. Kourkoutis, S. Muhlbauer, P. Boni, D. A. Muller, Y. Barash, J. Schubert, Y. Idezerda, J. Mannhart and D. G. Schlom, "Epitaxial intergration of the highly spin-polarized ferromagnetic semiconductor EuO with silicon and GaN", Nat. Mater., vol. 6, 882, (2007). 
[148] A. Gupta, X. W. Li and Gang Xiao, "Magnetic and transport properties of epitaxial and polycrystalline chromium dioxide thin films", J. Appl. Phys., vol. 87, 6073, (2000).

[149] X. W. Li, A. Gupta, Gang Xiao and G. Q. Gong, "Low-field magnetoresistive properties of polycrystalline and epitaxial perovskite manganite films”, Appl. Phys. Lett., vol. 71, 1124, (1997).

[150] A. Miele, R. Fletcher and E. Zaremba,Y. Feng, C. T. Foxon and J. J. Harris, "Phonondrag thermopower and weak localization”, Phys. Rev. B., vol. 58, 13181, (1998).

[151] F. J, Blatt, "Physics of electronic conduction in solids”, McGraw-Hill, New York, (1968).

[152] R. D. Barnard, "Thermoelectricity in Metals and Alloys”, Taylor \& Francis Ltd, (1972).

[153] D. K. C. MacDonald, “Thermoelectricity - An Introduction to the Principles”, Dover Publications, INC, (1961).

[154] F. J. Blatt, P. A. Schroeder, C. L. Foiles and D. Greig, “Thermoelectric Power of Metals", Plenum Press, New York and London, (1976).

[155] Shivkant Singh, Milad Yarali, Shahab Shervin, Venkat Venkateswaran, Kathy Olenick, John A. Olenick, Jae-Hyun Ryou and Anastassios Mavrokefalos, “Temperaturedependent thermal conductivity offlexible yttria-stabilized zirconia substrate via $3 v$ technique”, Phys. Status. Solidi A., vol. 10, 1700069 (2017).

[156] J. W. Gerlach, J. Mennig and B. Rauschenbach, "Epitaxial gadolinium nitride thin films”, Appl. Phys. Lett., vol. 90, 061919, (2007).

[157] A. L. Koo, "Optoelectronic and Structural Properties of nx-GaN, a-GaON and GaMnN”, Ph.D. thesis, Victoria University of Wellington, (2005).

[158] A. Le. Louarn, S.Vézian, F. Semond and J.Massies, “AlN buffer layer growth for GaN epitaxy on (1 1 1) Si: Al or Nfirst?”, Journal of Crystal Growth, vol. 311, 3278, (2009).

[159] B. D. Cullity, "Elements of X-ray Diffraction”, 2nd ed, Addison-Wesley Publication Company, INC, (1978).

[160] J. R. Tesmer and M. A. Nastasi, "Handbook of modern ion beam materials analysis", Materials Research Society, (1995). 
[161] M. Mayer, “Rutherford Backscattering Spectrometry (RBS)”, Workshop on Nuclear Data for Science and Technology: Materials Analysis, Trieste, (2003).

[162] W. K. Chu, J. W. Mayer and M. A. Nicolet, “Backscattering Spectrometry”, Academic Press, New York, (1978).

[163] J. R. Bird and J. S. Williams, “Ion beams for materials analysis”, Academic Press, (1989).

[164] L. R. Doolittle, "Algorithms for the rapid simulation of Rutherford backscattering spectra”, Nucl. Instr. Meth., vol. 9, 344 (1985).

[165] M. McElresh, “Fundamentals of magnetism and magnetic measurements”, Quantum Design, Inc., MPMS, (1994).

[166] R. L. Fagaly, "Superconducting quantum interference device instruments and applications”, Rev. Sci. Instrum., vol. 77, 101101, (2006).

[167] Physical Property Measurement System - Hardware Manual, Quantum Design Inc., Sixth edition, (2008).

[168] R. Green, “Hall Effect Measurements in Materials Characterization”, Keithley Instruments, Inc. (2011).

[169] Physical Property Measurement System - AC Transport Option User's Manual, Quantum Design Inc., Fifth edition, (2003).

[170] Instructions - Silicon Diode and GaAlAs Diode Temperature Sensor Installation, Lake Shore Cryotronics, (2013).

[171] A. Mawdsley, "Thermoelectric Power of Amorphous Metals and High-Temperature Superconductors”, Ph.D. thesis, Victoria University of Wellington, (1988).

[172] K. D. D. Rathnayaka, “Thermoelectric power of Manganin”, J. Phys. E: Sci. Instrum., vol. 18, (1985).

[173] A. Punya, T. Cheiwchanchamnangij, A. Thiess and W. R. L. Lambrecht, "Firstprinciples study of nitrogen vacancies in GdN”, MRS Fall Meeting, (2010).

[174] P. G. de Gennes and J. Friedel, “Anomalies de resistivite dans Certains metaux magnetiques”, J. Phys. Chem. Solids., vol. 4, 71, (1958). 
[175] M. E. Fisher and J. S. Langer, "Resistive anomalies at magnetic critical points", Phys. Rev. Lett., vol. 20, 665, (1968).

[176] M. R. Oliver, J. O. Dimmock, A. L. McWhorter and T. B. Reed, "Conductivity Studies in Europium Oxide”, Phys. Rev. B., vol. 5, 1078, (1972).

[177] A. Sharma and W. Nolting, "Additional carrier-mediated ferromagnetism in GdN", Phys. Rev. B., vol. 81, 125303, (2010).

[178] J. M. D. Coey, M. Venkatesan and C. B. Fitzgerald, "Donor impurity band exchange in dilute ferromagnetic oxides”, Nat. Mater., vol. 4, 173, (2005). 